Prepared for the U.S. Department of Energy

under Contract DE-AC05-76RL01830

\title{
In Vivo Monitoring Program Manual, PNL-MA-574
}

TP Lynch

July 2010

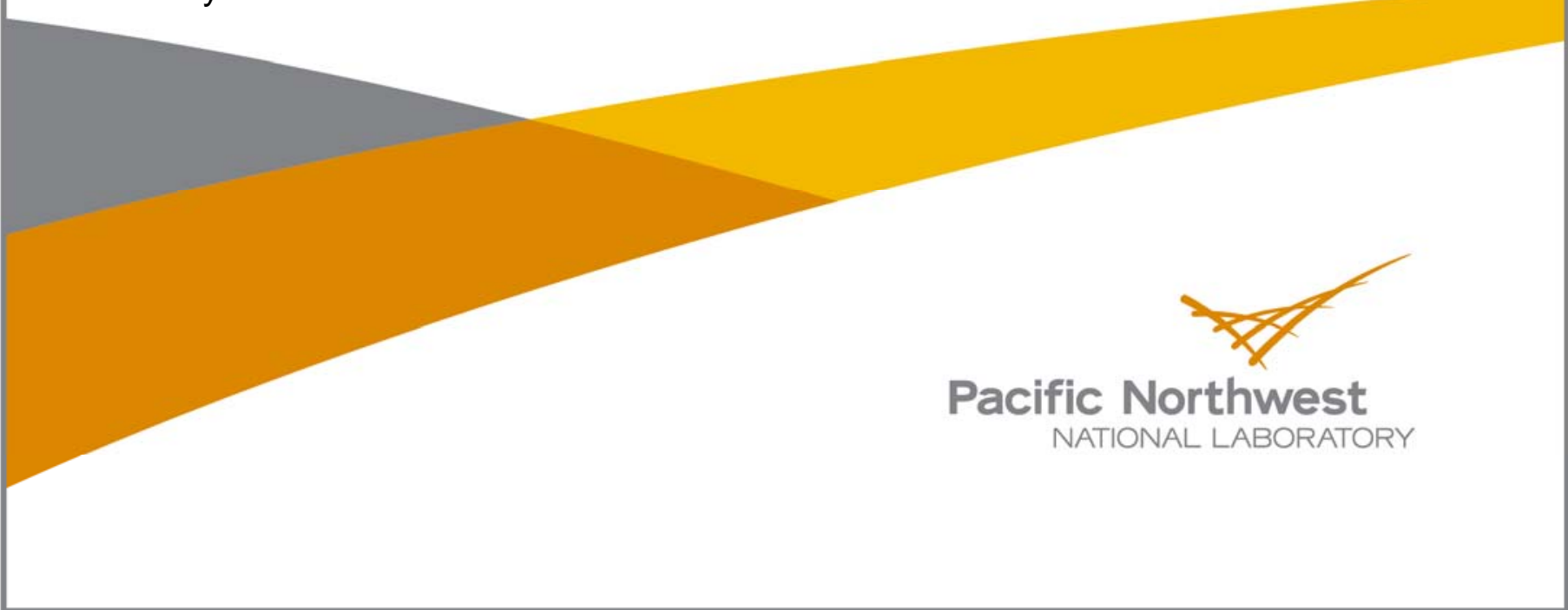


PNNL-19516, Rev. 5

\section{In Vivo Monitoring Program Manual, PNL-MA-574}

TP Lynch

July 2010

Prepared for

the U.S. Department of Energy

under Contract DE-AC05-76RL01830

Pacific Northwest National Laboratory

Richland, Washington 99352 


\section{Radiation and Health Technology}

\section{IN VIVO MONITORING PROGRAM MANUAL}

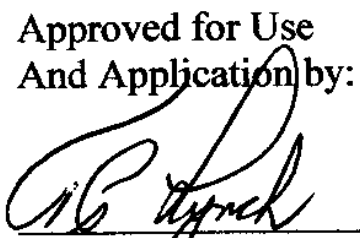

T. P. Lyuch, Program Manager

In Vivo Monitoring Program

Radiation and Health Technology

8.c Baka.

S. C. Baker, Manager

Radiation and Health Technology

Battelle

Pacific Northwest National Laboratory

Richland, Washington 99352 
“ALL PRINTED COPIES OF PNL-MA-574 ARE VALID ONLY FOR THE DATE PRINTED”

(If you attempt to print procedures and encounter difficulties, call Ronda Biaggi @ 372-1747.)

IN VIVO MONITORING PROGRAM MANUAL PNL-MA-574, REV. 5

Table OF Contents

1.0 Program Administration

2.0 Facilities and Equipment

In Vivo Measurement Systems

4.0 In Vivo Measurement Protocols

5.0 Quality Assurance

6.0 In Vivo Measurement Data Processing and Analysis

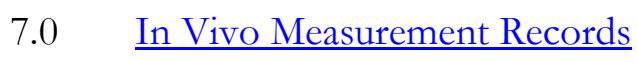

Appendix A - History of Count Types and Minimum Detectable Activity

Appendix B - Historical Summary of Count Types, Body Codes, and Detector Codes

Appendix C - The Hanford In Vivo Radiobioassay and Research Facility Pamphlet

Appendix D-Acronyms 
IN VIVO MONITORING PROGRAM MANUAL

PNL-MA-574

SECTION 1.0

PROGRAM ADMINISTRATION 


\section{Contents}

1.0 Program

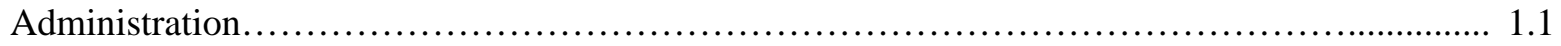

1.1 Organizational Structure, Planning, and Reporting..................................... 1.1

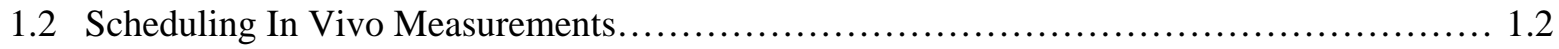

1.3 Performing In Vivo Measurements.................................................... 1.3

1.3.1 In Vivo Count Record Form.................................................. 1.3

1.3.2 Ensuring Measurement of Internally Deposited Radioactive Material............... 1.5

1.3.3 Information and Safety Instructions for the Subject............................ 1.5

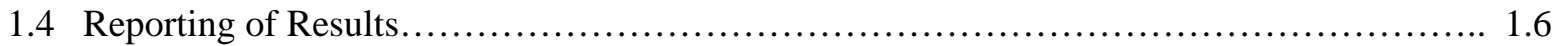

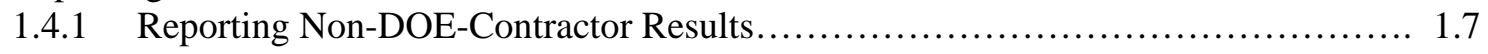

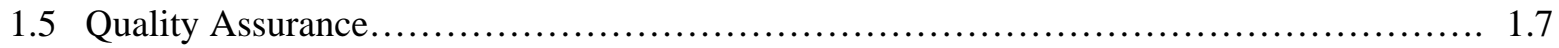

1.6 Cited References............................................................. 1.7 


\subsection{Program Administration}

The following sections provide an overview of the administration for the In Vivo Monitoring Program (IVMP) for Hanford. This includes the organizational structure and program responsibilities; coordination of in vivo measurements; scheduling measurements; performing measurements; reporting results; and quality assurance.

\subsection{Organizational Structure, Planning, and Reporting}

Overall responsibility for the management of the IVMP rests with the Program Manager (PM). The $\mathrm{PM}$ is responsible for providing the required in vivo counting services for Hanford Site contractor employees in accordance with Department of Energy (DOE) requirements and the contractor statements of work.

To help in meeting this responsibility, the PM oversees the following administrative tasks:

Plan for an adequate level of technical resources to support the program including staff, equipment, and facilities.

Costs are monitored and compared against recovery to identify variances from the anticipated budget. Steps are taken to minimize the variance from the projected spending rate. Reports are routinely prepared for the contractors that summarize the number, types, and results from the in vivo measurements.

Additional reports are prepared as requested to inform Pacific Northwest National Laboratory (PNNL) management, other contractors, and the DOE, Richland Operations Office, Office of River Protection, and the Pacific Northwest Site Office about the status of the program, including problems and highlights.

Information on the number and types of counts performed is routinely submitted to R\&HT Administration for proper costing of the services.

Provide technical direction for the program.

The responsibility for the daily operations of the IVMP is delegated to the Operations Supervisor (OS). The OS oversees the work of the technicians and support personnel to ensure that work is performed in accordance with the clients' requirements and the established policies and procedures. The OS reports directly to the PM. More detailed information on the roles and responsibilities of the program staff is contained in PNL-MA-574, In Vivo Monitoring Program Procedures Manual, procedure IVMP100.A.05, Organization of the In Vivo Monitoring Program.

Figure 1.1 illustrates the IVMP organizational structure. The supporting staff (e.g., Craft Services and Radiation Protection Technicians) are from different organizations within PNNL. Technical support is obtained as needed. 


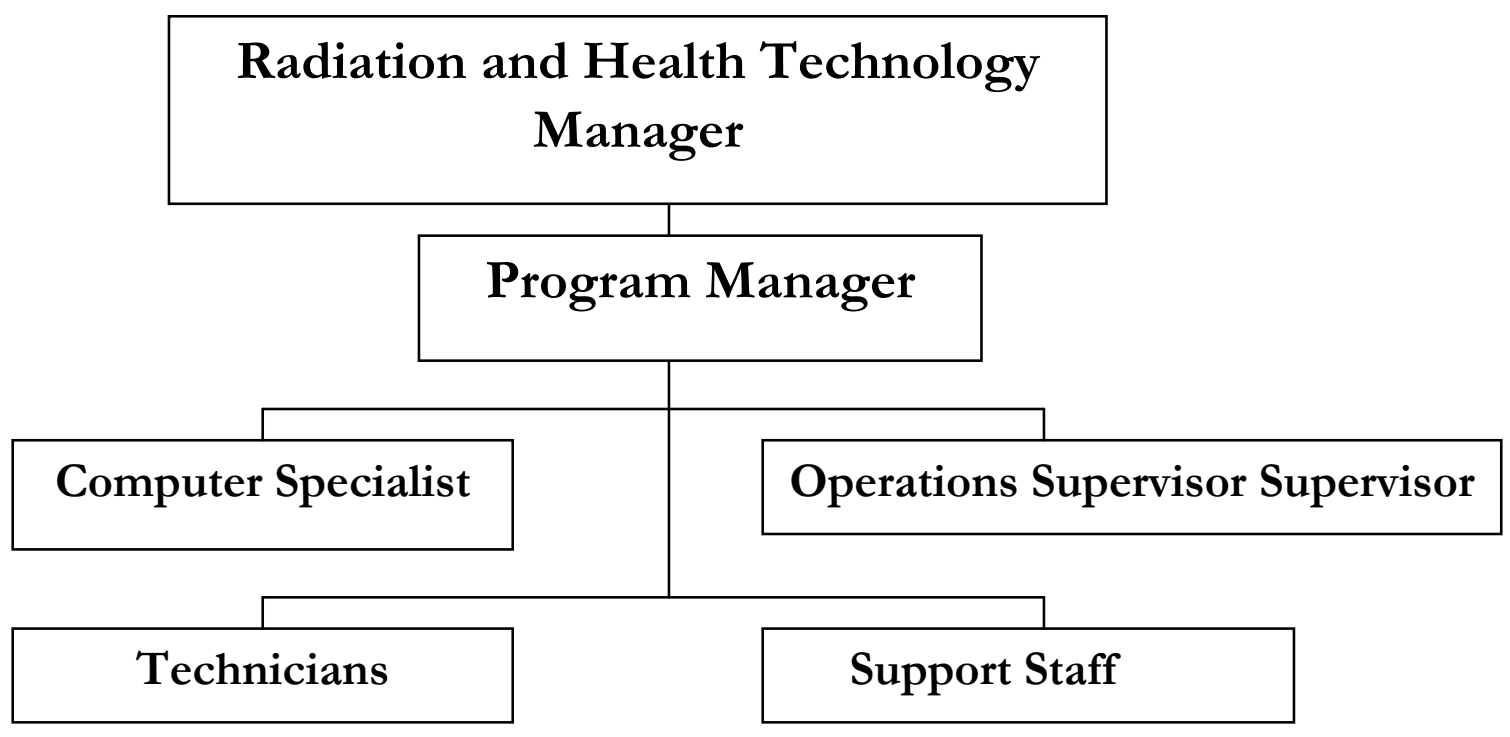

Figure 1.1. IVMP Organizational Structure

Changes are made to programmatic documents, measurement records, and software when new methods are developed and implemented, when new requirements are established, or when errors or deficiencies in practices or measurement results are identified. Changes to the information contained in this manual are made using Interim Change Notices (ICN) and revisions to the manual.

\subsection{Scheduling In Vivo Measurements}

Daily schedules for in vivo measurements are downloaded from the Hanford Radiological Exposure System (REX) database each morning. The Hanford contractors or PNNL Dosimetry Operations schedule the routine measurements. The IVMP staff coordinates with Dosimetry Operations, Internal Dosimetry, and the contractor schedulers to schedule workers for special and follow-up measurements.

Each contractor is responsible for determining the specific radionuclides their employees may be exposed to during work assignments. The contractor, often in consultation with the Internal Dosimetry Program (IDP), determines the type of in vivo measurement(s) required. These measurements are typically scheduled when an employee is hired, terminated, begins or ends a project requiring in vivo bioassay, or upon a request for a periodic routine measurement.

Each contractor scheduler has access to appointment times in the REX database to schedule the different types of counts. Daily schedules are downloaded to an IVMP computer over the network. The information contains demographic information and information on the type of count required.

The IVMP staff generates a daily file that is returned to REX. The file includes a list of the persons counted and the type of measurement(s) performed on a specific day. The file is available to the contractors through REX and is used as input to their radiological access control systems.

Ten-minute whole body measurements, lung measurements, and other organ measurements are scheduled for a specific date and time. The 3-minute whole body measurements are scheduled for a date but not for a specific time during the day. Contractors schedule the majority of whole body, lung and 
other organ counts using REX. Generally, the routine measurements are scheduled to about $90 \%$ of capacity to accommodate follow-up measurements. However, scheduling to capacity or extending operating hours is periodically done to handle peak demand periods. A reasonable attempt is also made to accommodate unscheduled measurements.

Workers that are not scheduled for measurements are discouraged from just walking in. However, measurements may be performed for unscheduled workers on a case-by-case basis if authorized by the contractor organization. If an individual arrives and is not listed on the daily schedule, the respective scheduling organization is contacted to make sure that person requires a count. If a measurement is needed, the individual is given the option to wait until they can be counted in between the scheduled measurements or to schedule for another day.

The IVMP staff work closely with the contractor scheduling organizations to make sure that the required in vivo counting services are provided in a timely manner. To assist the IVMP staff in accommodating the contractors' needs, the counts are prioritized, based on the reason for the count. Measurements for workers who have experienced an unplanned intake or suspected intake of radioactive material within 24-48 hours receive top priority.

The reasons why measurements are performed are listed below in order of decreasing priority and the list is used as a general guide to plan the order in which measurements are made.

1. Incident counts within the first two days after the incident

2. Recount of an unexpected positive result

3. Work termination counts

4. Anyone previously canceled

5. Scheduled DOE contractor counts

6. Anyone later than 30 minutes for a scheduled appointment

7. Non-DOE worker measurement.

The in vivo measurements for the non-DOE clients are scheduled to avoid conflict with the DOE measurements. When a conflict arises, the DOE measurement receives priority and the non-DOE measurement is delayed or rescheduled.

\subsection{Performing In Vivo Measurements}

The general sequence for performing an in vivo measurement includes: having the worker complete the In Vivo Count Record form, take initial steps to minimize interference from external contamination, positioning the worker in the proper geometry, performing the measurement, calculating results, doing an initial review to check the validity of the results, and providing a preliminary letter with a qualitative summary of the results to the worker. The current revision to PNL-MA-554 contains the detailed operating procedures for the different types of in vivo measurements.

\subsubsection{In Vivo Count Record Form}

When a worker arrives for a scheduled measurement, they will be listed on the daily schedule. The individual fills out the applicable sections of the In Vivo Count Record form. An example of the form is shown in Figure 1.2.

Issued: July 2010

PNNL-MA-574 Section 1.0

Supersedes: January 2007 
IN VIVO COUNT RECORD

PLEASE FILLIN THE INFORMATION BELOW

Last Name, First Full Name, MI

Social Security No.

\begin{tabular}{|l|l|}
\hline Payroll No. & Employer \\
\hline
\end{tabular}

\begin{tabular}{|l|l|l|l|l|}
\hline Birth Date (MM/DD/YY) & Height (in) & Weight (lbs) & Gender & Today's Date \\
\hline
\end{tabular}

\section{PLEASE READ AND ANSWER BEFORE RETURNING FORM}

- Are you claustrophobic?

- Have you had a nuclear medicine study or radlopharmaceutical injection within the past two months? (This does not include counts at this facility.)

- The technician has audio and visual communication with you while you are being counted. The technician will assist you in exiting the cell if necessary.

- For chest counts there is a signal button within reach to alert the technician. You may exit the cell if necessary. The technician will describe the self exit procedure to you before your count is started.

- I have read and understand the above statements.

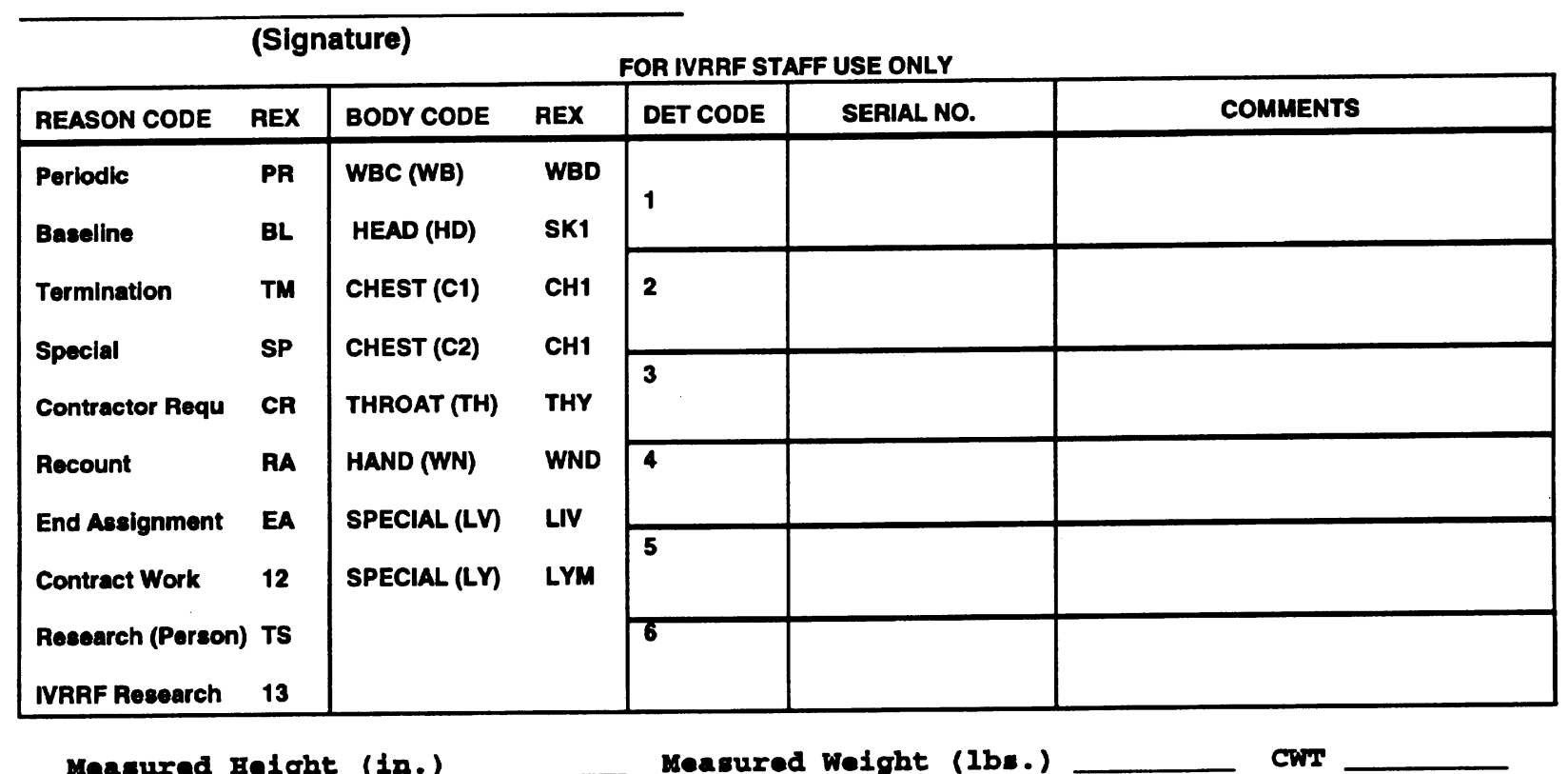

Figure 1.2. In Vivo Count Record Form 


\subsubsection{Ensuring Measurement of Internally Deposited Radioactive Material}

Precautions are taken to help ensure that only internally deposited radioactive material is measured and to limit the amount of radioactivity being transferred into the shielded enclosures.

For a routine whole body count, the subject removes ancillary items such as shoes, coat, hat, watch, cell phone, and pager. The measurement is usually performed with the worker in their street clothes unless otherwise directed by specific contractor requirements. If the results from the initial measurement show a detectable amount of radioactive material present, the subject showers and changes into a pair of cotton coveralls. This is done to remove trace amounts of radioactive material that may be present on the skin, hair, or clothing. The subject is then recounted.

For a routine lung count, the subject is provided with a pair of cotton coveralls and foot covers to wear during the initial measurement. If the result from the initial lung measurement shows a detectable amount of radioactive material, the worker is asked to shower and then is recounted while wearing coveralls. If the worker cannot be recounted immediately, they are rescheduled for another time or date.

When a subject has potential internal contamination from an incident that occurred within the past 24 hours, a personal contamination survey may be conducted prior to entering the counting room to prevent contamination of the room. In these cases, the subject usually showers and dons a pair of coveralls and foot covers before being counted.

\subsubsection{Information and Safety Instructions for the Subject}

The IVMP staff attempt to make those who arrive for counts feel as comfortable as possible during the measurement procedure. A pamphlet entitled, "The Hanford in Vivo Radioassay and Research Facility," describing the different types of measurements that are performed, is available in the lobby. (A copy of the pamphlet is included as Appendix C.)

For the routine standup whole body count: The subject is instructed to step into the counter for the measurement. He or she is typically told the length of the count and is told to step out of the counter when the count is complete.

For lung counts, other organ counts, and coaxial high purity germanium (HPGe) counts: When the subject is in an enclosed counting room for a measurement, the technician may provide some or all of the following information before starting the count:

the length of the count,

the use of the intercom and emergency button,

the use of the video camera for surveillance,

the self-exit procedure,

the need to remain still during the count.

The technician will also answer any additional questions a subject may have about the procedure. A very small percentage of workers who receive counts in the shielded rooms indicate they are 
uncomfortable in enclosed spaces. These persons can be counted with the door partially open. This may slightly elevate the detection level for the measurement. A comment is placed in the measurement record to indicate the door was open during the measurement.

\subsection{Reporting of Results}

The electronic files of the in vivo measurement results are routinely transmitted to REX. This is typically accomplished within 5 working days after a count is completed. Each contractor may then access the in vivo measurement results for their employees through REX. Results can also be obtained directly from the IVMP staff right after a measurement is completed. Result sheets containing the in vivo measurement results are maintained as program records. The routine quality control data, calibration data, and other supporting information are also maintained as program records.

At the completion of most in vivo measurements, the subject is given a letter that summarizes the measurement results and provides the contact information for the contractor's dosimetry representative. An example of the format of a typical letter is shown in Figure 1.3. If requested, the IVMP staff or Internal Dosimetry staff will discuss the preliminary results with the worker and answer any questions. Workplace questions are typically referred to the contractor dosimetry representative listed on the letter given to the subject.

The REX database that contains the electronic files with the measurement results is maintained by the Hanford Radiological Records Program daily. The signed paper copies of the individual result sheets are ultimately transferred to Records Management (RM). There are no special notifications made for routine measurement results (i.e. periodic, contractor request, ending assignment, termination) that are below the detection level unless specifically requested by the contractor. The results from measurements related to a potential intake and results from follow-up measurements when a result from an initial measurement was above the detection level are promptly conveyed to Internal Dosimetry. 


\section{PRELIMINARY ANALYSIS OF IN VIVO EXAMINATION}

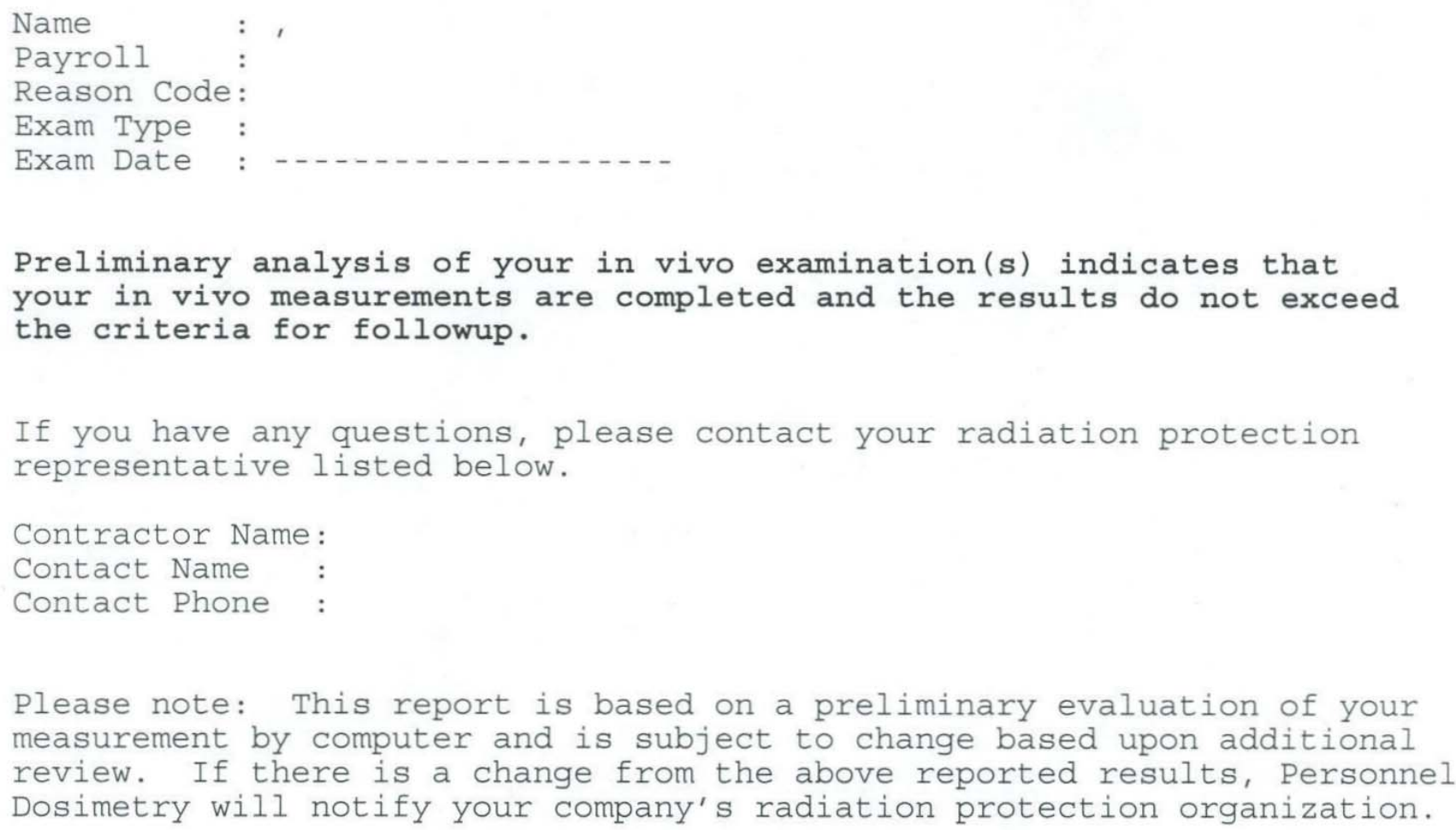

Figure 1.3. Example Format of Preliminary Results Letter

\subsubsection{Reporting Non-DOE-Contractor Results}

Reports of the measurement results are mailed to the company representative typically within two weeks after the measurement. Results at or above contractually specified levels are communicated promptly to the company representative. Any questions regarding results are referred to the OS or PM.

\subsection{Quality Assurance}

The IVMP is operated under an approved Radiation \& Health Technology (R\&HT) Quality Assurance Program Plan (QAPP). The QAPP addresses the quality assurance program requirements for the Dosimetry Services programs with program specific information in the appendices. A more detailed discussion of the overall program quality assurance (QA) program is found in Chapter 5.0.

\subsection{Cited References}

Pacific Northwest National Laboratory (PNNL). PNL-MA-554. R\&HT In Vivo Monitoring Program Procedures Manual. Richland, Washington. (Internal manual)

Radiation and Health Technology Quality Assurance Program Plan. Richland, Washington. (Internal Manual) 
IN VIVO MONITORING PROGRAM MANUAL

\author{
PNL-MA-574
}

SECTION 2.0

FACILITES AND EQUIPMENT 


\section{Contents}

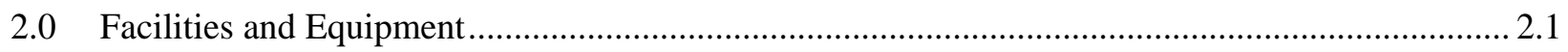

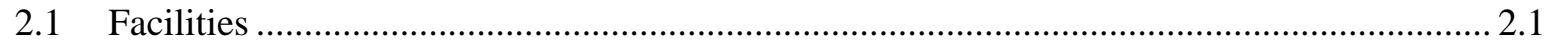

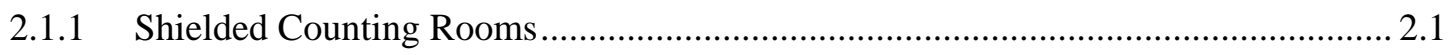

2.1.1.1 Palmer Room ..................................................................................... 2.1

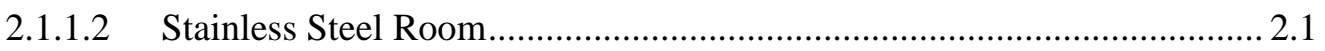

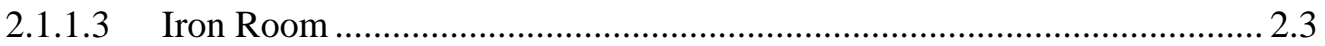

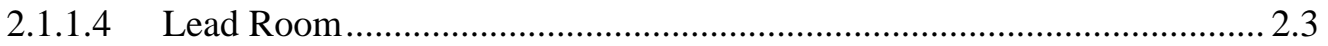

2.1.1.5 Standup Whole Body Counter .......................................................... 2.4

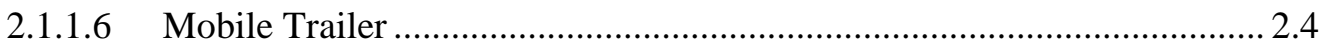

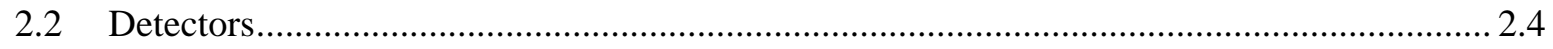

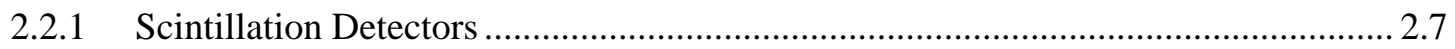

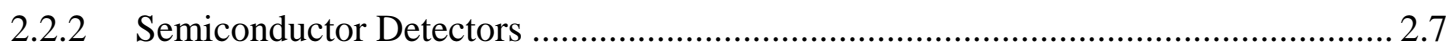

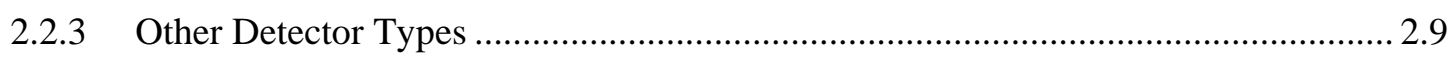

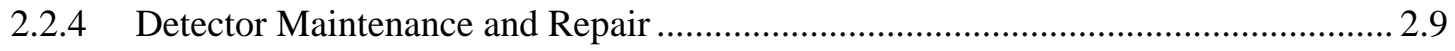

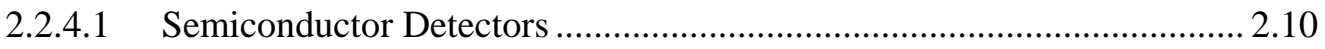

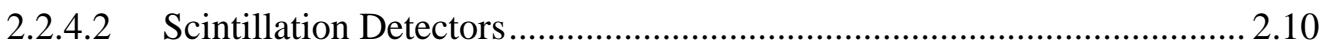

2.2.4.3 Determination of Detector Resolution ................................................ 2.10

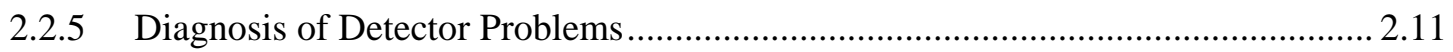

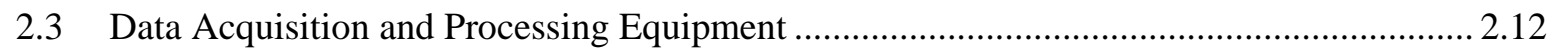

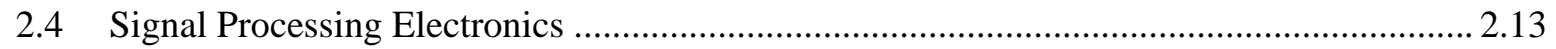

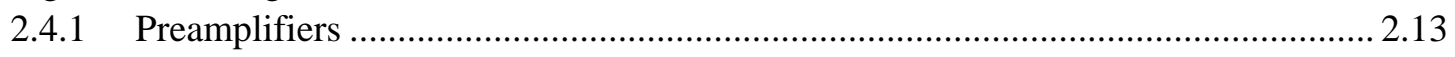

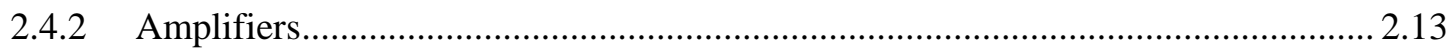

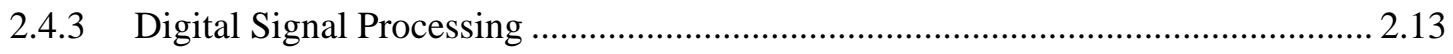

2.5 Spectroscopy Equipment: Multichannel Analyzer …....................................................... 2.14

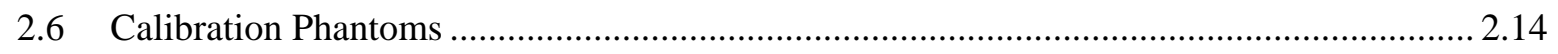

2.6.1 Bottle-Manikin Absorption Phantom ............................................................... 2.14

2.6.2 Tissue-Equivalent Torso Phantoms .................................................................. 2.17

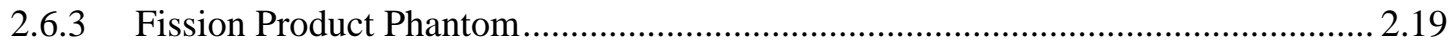

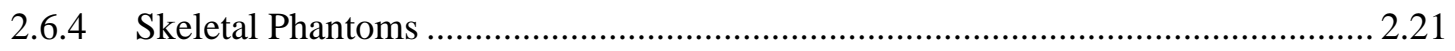

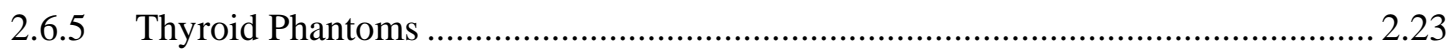

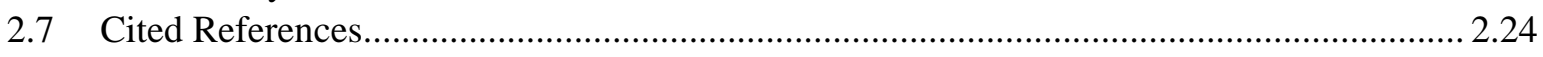

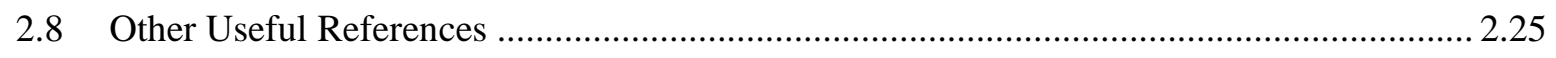




\subsection{Facilities and Equipment}

The In Vivo Monitoring Program (IVMP) maintains the facilities and equipment to provide quality in vivo measurement services in support of the Hanford mission. The In Vivo Radiobioassay and Research Facility (IVRRF) located in Richland, Washington, houses the shielded counting rooms, measurement systems and support areas used to perform the in vivo measurements. The building is designated as 747A. Figure 2.1 and Figure 2.2 show the IVRRF reception area and the primary technician workstation, respectively. A semi-trailer contains additional measurement systems.

\subsection{Facilities}

The in vivo measurement systems are shielded to significantly reduce the natural background radiation levels associated with building materials, soil, air, and cosmic rays. The shielded rooms reduce the background radiation by a factor of 50 to 100, depending on the energy of the radiation. The IVMP maintains five shielded counting systems and a portable gamma spectrometry system in the 747 A Building. Two shielded measurement systems are in standby mode and can be assembled as needed in a mobile trailer that is located near the 747A Building. More detailed descriptions of in vivo measurement system operations are found in Chapter 3.0.

\subsubsection{Shielded Counting Rooms}

The layout of the five shielded counting rooms in the 747A Building is shown on the floor plan in Figure 2.3. The walls, floor, and ceiling for three rooms are made of hardened steel from decommissioned battleships. A common ventilation supply system services the Palmer and Stainless Steel Rooms and a second system services the Iron room and Lead Room. Each of these rooms also contains a video camera and intercom system. The video output and intercom are monitored by IVMP staff during the measurements. The rooms are described here in the order of their size.

\subsubsection{Palmer Room}

The Palmer Room dimensions are 2.5 × 3.7 × 2.4 m ( 8.3 x 12 x 8 ft), with the walls, floor, and ceiling made from 30-cm (12-in.) thick battleship armor plate from the U.S.S. Indiana. The inside surfaces are lined with a graded shield of lead (3 mm), cadmium $(1 \mathrm{~mm})$, and copper $(1 \mathrm{~mm})$. The shield door is opened and closed manually. This room was moved to Richland from the University of Utah in 1988 and put into service in June 1989. A five-detector array of large volume coaxial high purity germanium (HPGe) detectors is installed in the room for whole body measurement of gamma-ray energies above 200 $\mathrm{keV}$. The five detectors are mounted on a platform that is driven by a motion control system. The detectors move from the head to the knees of the supine subject during the count.

\subsubsection{Stainless Steel Room}

The Stainless Steel Room dimensions are 2.9 × 3.0 × $2.2 \mathrm{~m}(9.5 \times 10 \times 7.25 \mathrm{ft})$. It has common walls with two other shielded cells. It has a 30-cm (12-in.) thick iron wall in common with the Palmer Room, a 25.4-cm (10-in.) thick iron wall in common with the Iron Room, and two iron walls 19-cm (7.5-in.) thick from the Nevada Test Site. The shield door is mounted on rollers, is counter-weighted, and pneumatically operated. The floor and ceiling are composed of 12.5-cm (5-in.) thick iron armor plate and 10-cm (4-in.) of lead brick. This room has a graded shield of 0.318-cm (0.125 in.) lead, 0.159-cm (0.0625-in.) tin, and 0.159-cm (0.0625-in.) stainless steel. 


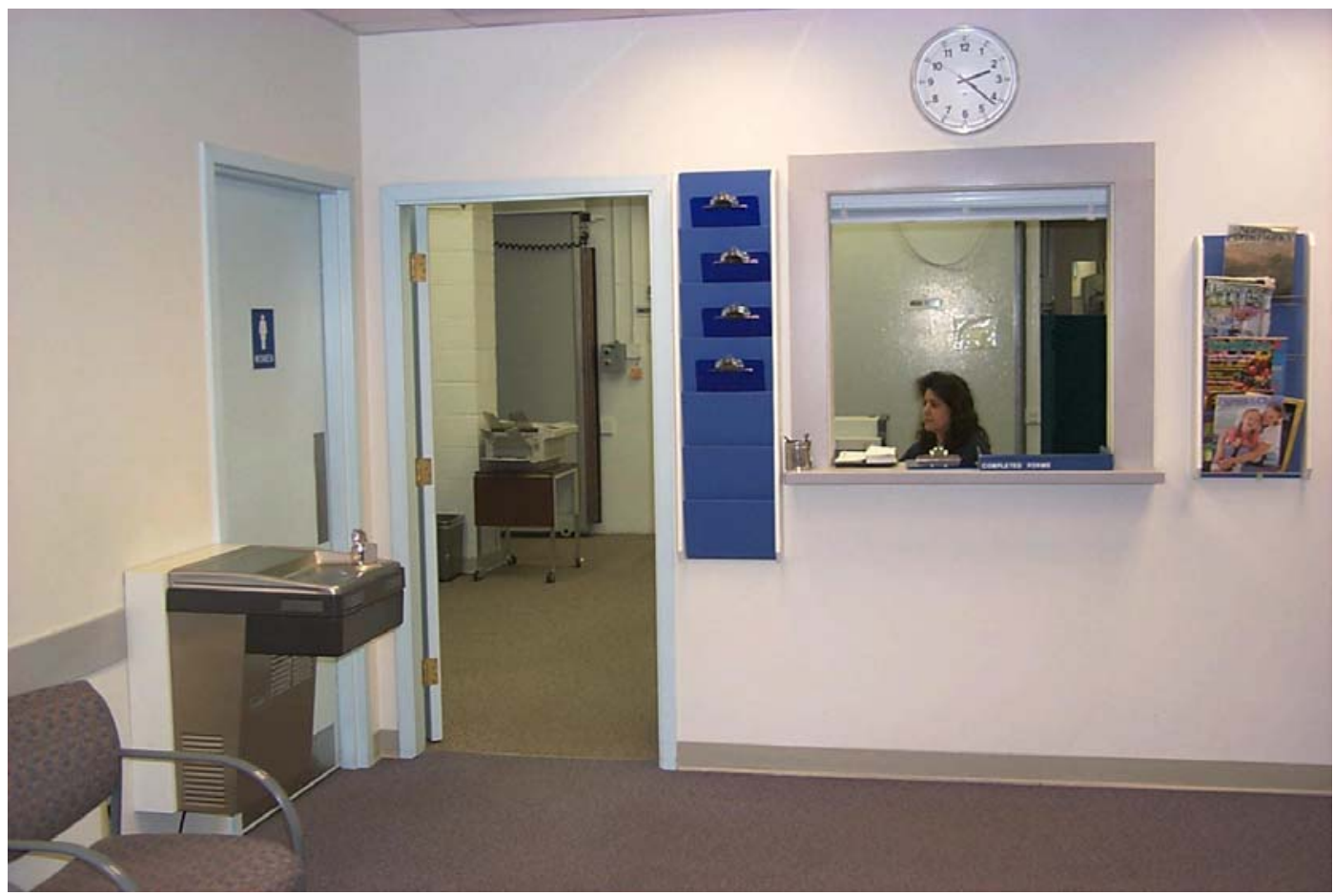

Figure 2.1. IVRRF Waiting Room and Reception Area

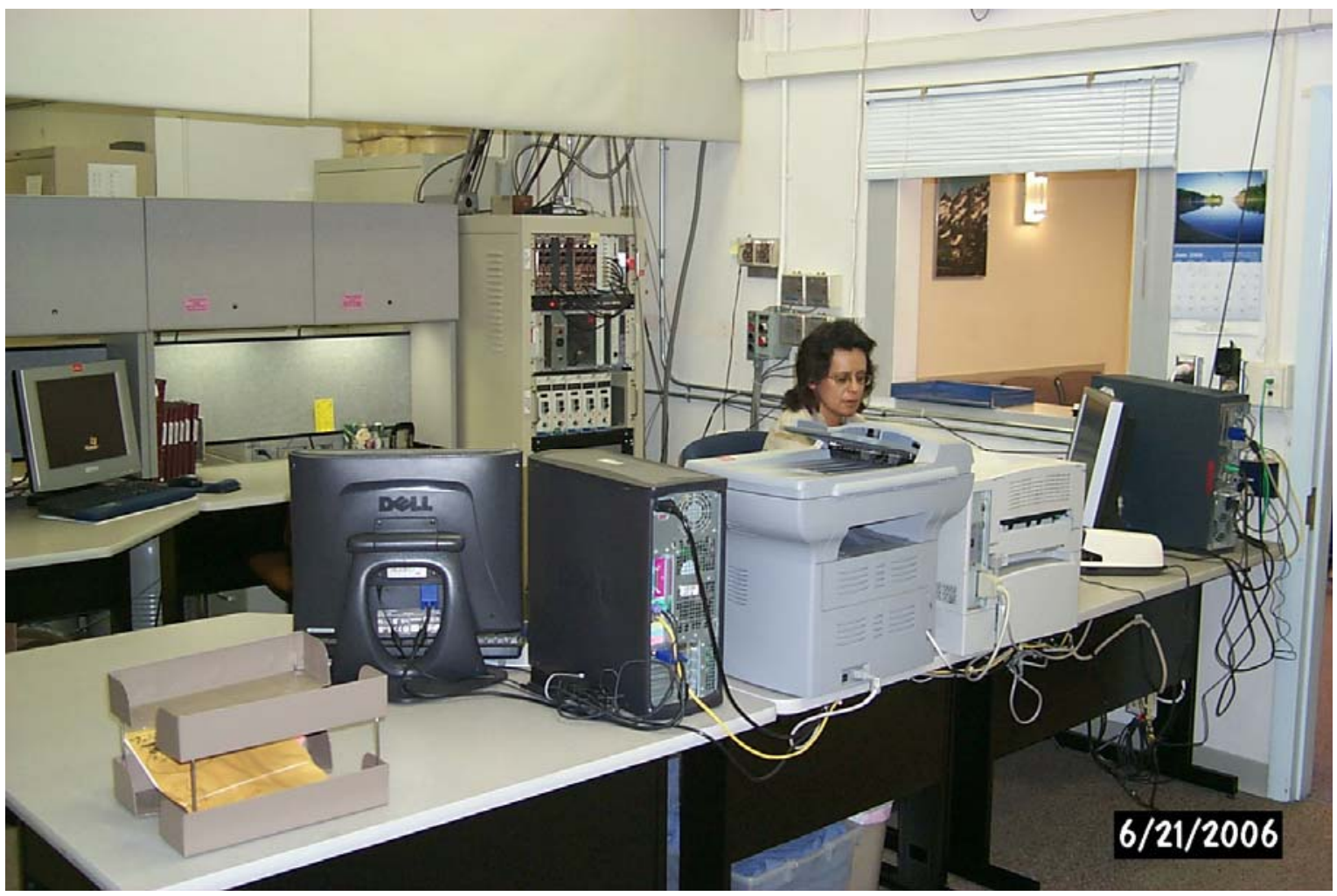

Figure 2.2. IVRRF Technician Workstation 


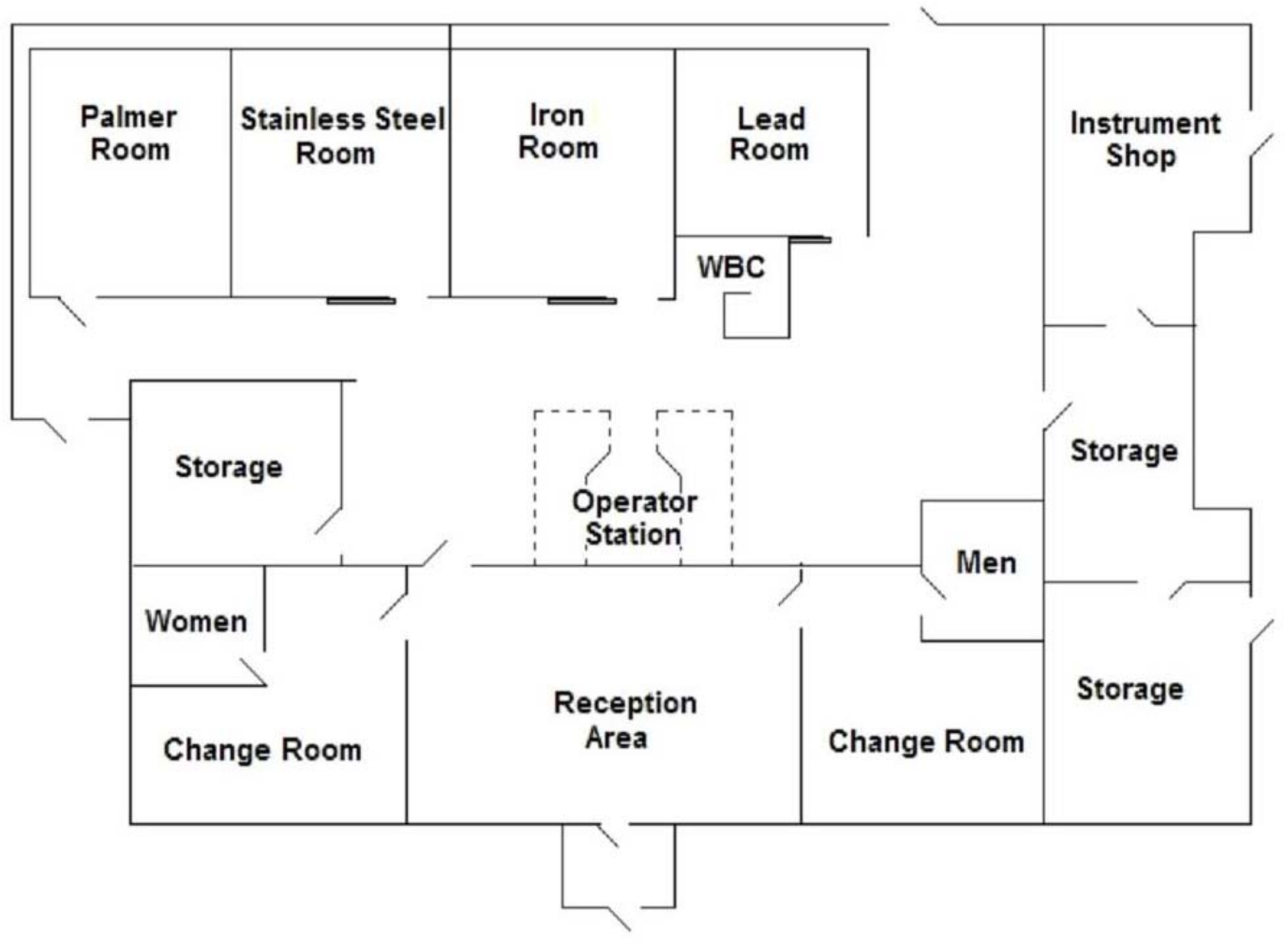

Figure 2.3. Floor Plan of the 747A Building

The graded shield components are somewhat different compared to the Iron Room. Tin was used instead of cadmium because it was cheaper at the time, and the stainless steel was used instead of copper because of its better wear properties. Background measurements taken in the room indicated that this type of shielding was equivalent to the Iron Room shielding. An array of four $38-\mathrm{cm}^{2}$ planar HPGe detectors is mounted in this room and is used primarily for measuring americium and uranium in the lungs.

\subsubsection{Iron Room}

The Iron Room dimensions are 2.7 x 3.0 x 2.3 m (8.9 x 9.8 x $7.3 \mathrm{ft})$, having 25.4-cm (10-in.) thick iron walls, ceiling, and floor. The shield door is opened and closed using an electrically driven screw drive mechanism. An array of four $38-\mathrm{cm}^{2}$ planar HPGe detectors is mounted in this room and is used primarily for measurements of americium and uranium in the lungs. These detectors have a similar cryostat design as the detectors used in the Stainless Steel Room and are arranged in a similar configuration.

A graded shield of 3.2-mm (0.125-in.) lead, 0.5-mm (0.02-in.) cadmium, and 1.5-mm (0.06-in.) copper comprises the inner lining of the room to further reduce the scattered background radiation at low energies. For further details on the construction of this room, see Hanford Whole Body Counter 1959 Activities (1960).

\subsubsection{Lead Room}

The Lead Room has 10-cm (4-in.) thick lead walls, ceiling, and floor composed of virgin lead bricks that are covered by a $1.0-\mathrm{mm}(0.04$-in.) thick layer of copper. The room is $2.85 \mathrm{~m}$ long x $2.34 \mathrm{~m}$ wide $\mathrm{x}$ 
$2.08 \mathrm{~m}$ high (9 ft 4 in. x $7 \mathrm{ft} 8 \mathrm{in.} x 6 \mathrm{ft} 10 \mathrm{in}$.). The shield door is opened and closed using an electrically driven screw drive mechanism. An array of two $38 \mathrm{~cm}^{2}$ planar germanium detectors is used for routine measurements of uranium in the lungs. This system is also configured for thyroid, skeletal, and wound measurements. The thyroid and wound measurements are typically made with a single $38 \mathrm{~cm}^{2}$ planar germanium detector. The skeletal measurements are made with two detectors placed over the forehead.

\subsubsection{Standup Whole Body Counter}

The sodium iodide (NaI) detectors used in the standup counter are shielded with 10-cm (4-in.) thick lead brick. The shield is designed to allow only unscattered photons originating from the worker to reach the detectors. The system is designed for a subject to stand upright with a slight backward tilt during the measurement. The counter is a vertical shadow-shield configuration that is designed to allow unscattered photons coming only from the subject to reach the detectors. The steel frame holding the vertical detector array of five detectors is connected with a steel cable to a counter weight. This allows height adjustments of the array to match the height of the worker. This counter is used to perform screening measurements for radionuclides that emit photons with energies greater than $300 \mathrm{keV}$.

\subsubsection{Mobile Trailer}

Whole body counting systems are housed in a mobile trailer and are designed to measure high-energy gamma rays emitted from the body. Figure 2.4 is an exterior view of the mobile trailer. The unit contains two styles of shadow shielded counting systems. The horizontal shadow shield counter contains a 29-cm (11.5-in.) diameter $\mathrm{NaI}(\mathrm{Tl})$ scintillation detector. The counting platform for this shadow shield counter is raised 30.5-cm (12-in.) off the floor to provide easier entry to and exit from the counter.

In this style of shadow shield counter, a shield surrounds all but one side of the detector. The subject is in front of this open side. A shield behind the subject, the "shadow shield," is large enough and placed so that no photon traveling in a straight line from that side can enter the detector without passing through the shield. This shield "casts a shadow" over the opening in the detector shield. The only photons that can reach the detector without attenuation by the shield are those that are emitted from the subject or the shielding material and those scattered from the subject or the shadow shield.

There is also a standup $\mathrm{NaI}(\mathrm{Tl})$ whole body counter that can hold an array of six $\mathrm{NaI}(\mathrm{Tl})$ scintillation detectors in a vertical shadow-shield configuration. The detector array is fixed and does not allow height adjustment. Five of the detectors are $10 \times 10 \times 40 \mathrm{~cm}$ ( 4 × 4 × 16 in.) and the sixth, used for the head area, is $10 \times 10 \times 20 \mathrm{~cm}$ ( $4 \times 4 \times 8 \mathrm{in}$.). The array is $165 \mathrm{~cm}$ (66 in.) tall and is surrounded by 10-cm (4in.) of lead brick enclosed in 0.5-cm (3/16-in.) thick steel. This unit is currently in a standby mode.

The trailer can be powered by existing $115-\mathrm{V}$ or $230-\mathrm{V}$ commercial power or by a portable power generator. Germanium detectors can also be deployed in the trailer for wound and organ measurements.

More detailed information on the shadow shield unit is available in two reports that are available in the IVMP Library (Swanberg 1963; Eichner 1969).

\subsection{Equipment}

The IVMP maintains the instrumentation and equipment required to perform the different types of in vivo measurements required by the Hanford contractors. This section describes the major pieces of equipment and instrumentation used for the measurements. The IVMP staff maintains 
spare instruments and components, onsite instrument repair capability, and computer hardware and software maintenance contracts to help ensure continuity of operations.

\subsection{Detectors}

Scintillation and semiconductor detectors are the two main types of detectors used to perform the in vivo measurements at the IVRRF. The text by G. F. Knoll (2000), Radiation Detection and Measurement,

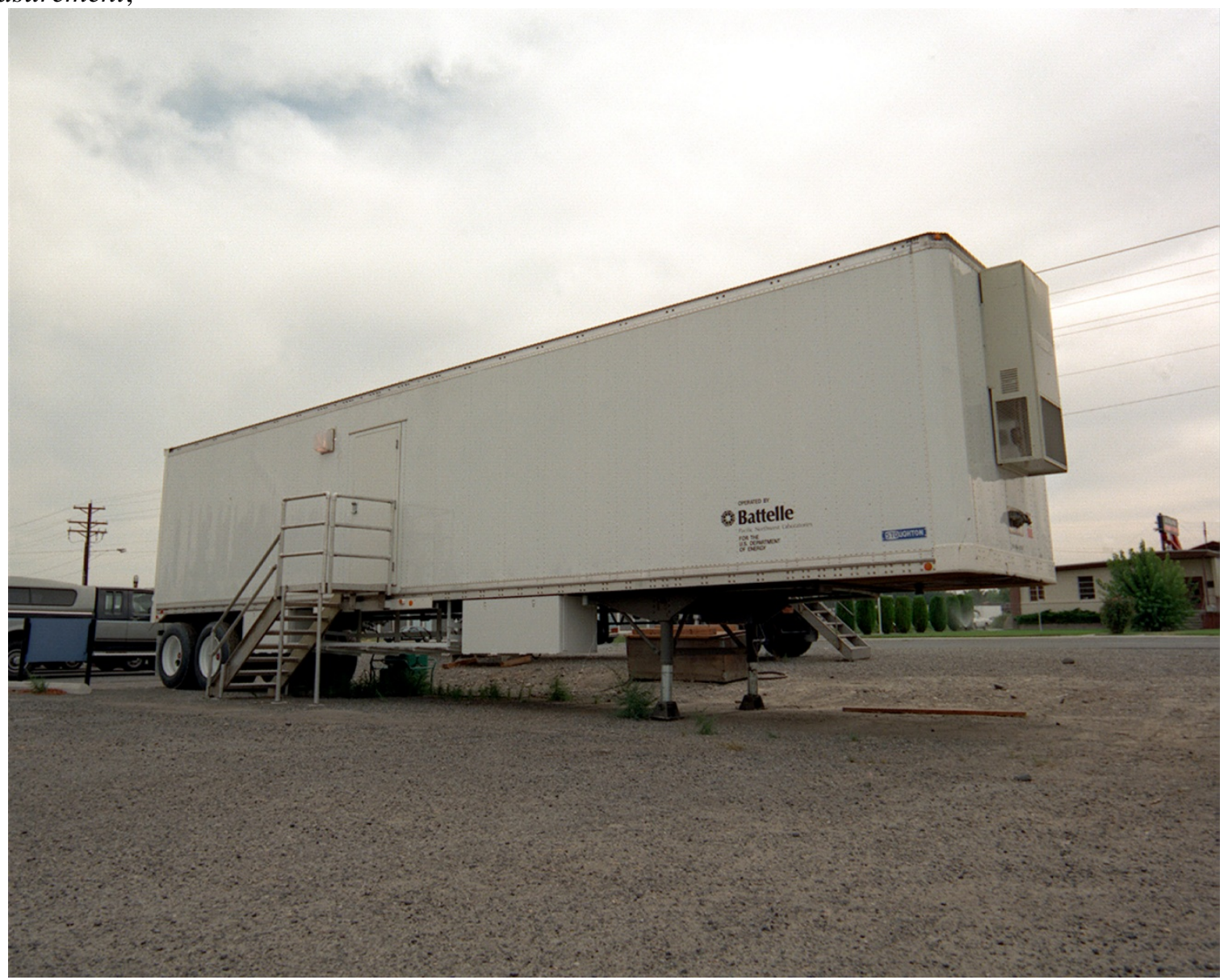

Figure 2.4. Mobile Whole Body Counter

and other sources can be consulted for a more detailed description of these detector types. In this section, the operation, design features, maintenance, and repair of the detectors used at the IVRRF are discussed.

Both types of detectors require periodic maintenance and routine monitoring to ensure proper performance. High voltage is required for the operation of both types of detectors. The scintillation detectors require positive high voltage, typically $800 \mathrm{~V}$ to $1200 \mathrm{~V}$; the semiconductor detectors require $2000 \mathrm{~V}$ to $5000 \mathrm{~V}$ and the polarity can be positive or negative depending on the type of semiconductor detector. 
The scintillation and semiconductor detectors are connected to high voltage supplies and signalprocessing instrumentation, as depicted in Figures 2.5 and 2.6, respectively. Sections 2.3 and 2.4 contain specific information about the data acquisition and signal processing electronics. 


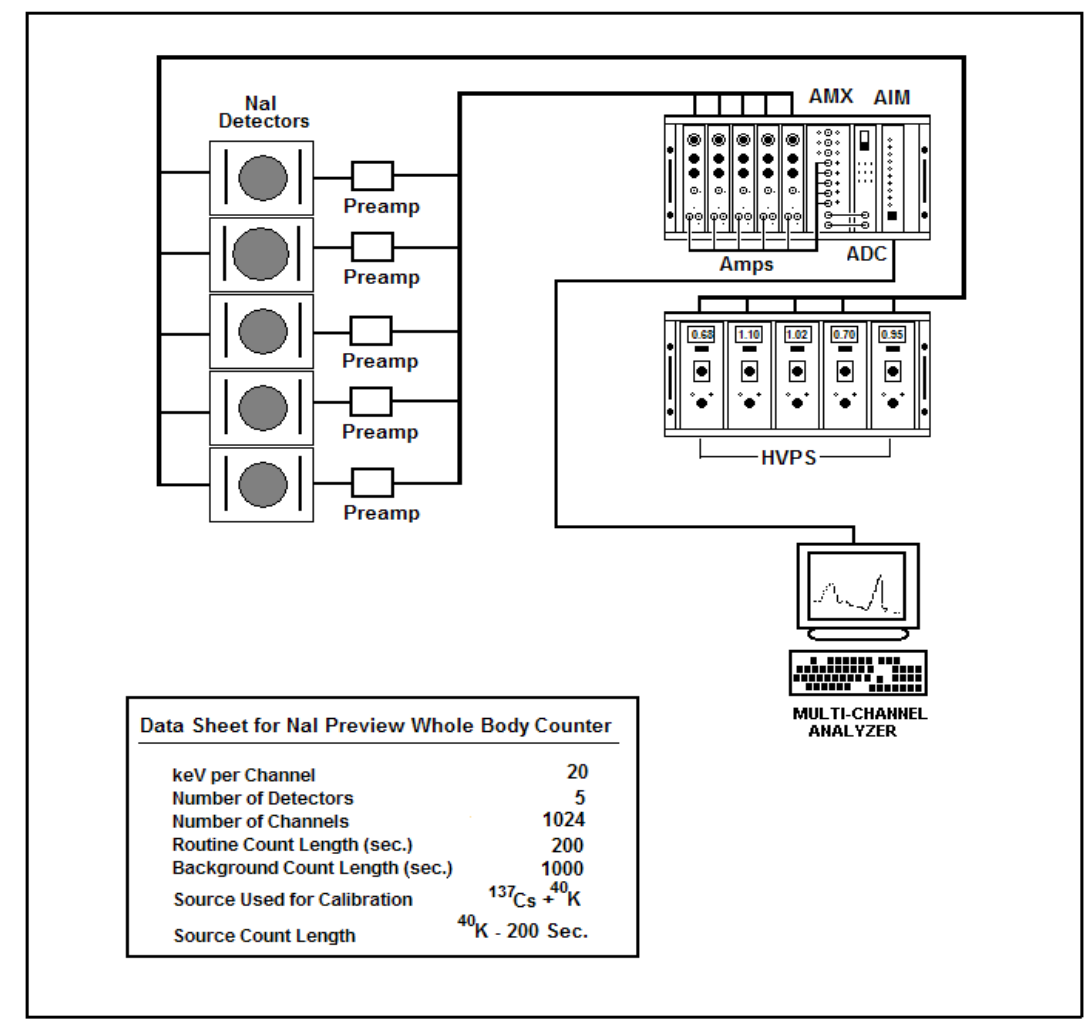

Figure 2.5. Scintillation Detector Signal Processing Diagram

\subsubsection{Scintillation Detectors}

Various sized scintillation detectors are available at the IVRRF. They include NaI(Tl) crystals ranging in size from 1-mm (0.04-in.) thick and 2.5-cm (1-in.) in diameter to 15.2-cm (6-in.) thick and 29.2-cm (11.5-in.) in diameter. Only the larger detectors are in routine use as described in Section 3.1.1. The larger crystals are optically coupled with three or four photomultiplier tubes per detector to optimize light collection.

The smaller NaI detectors can be used for wound measurements, thyroid measurements, lymph node, and other measurements. Several have thin entrance windows for detection of low energy photons. Currently, these smaller NaI detectors serve as backups to the germanium detectors.

\subsubsection{Semiconductor Detectors}

Two types of semiconductor detectors are used for routine in vivo counting. The planar HPGe detectors are used to measure low-energy photons over the energy range from $10 \mathrm{keV}$ to $500 \mathrm{keV}$. The coaxial HPGe detectors are used to measure higher-energy photons over the energy range of $200 \mathrm{keV}$ to 3 $\mathrm{MeV}$. Both types of the semiconductor detectors are operated at liquid nitrogen temperatures and provide excellent resolution. The semiconductor diodes are configured in various cryostat arrangements. 


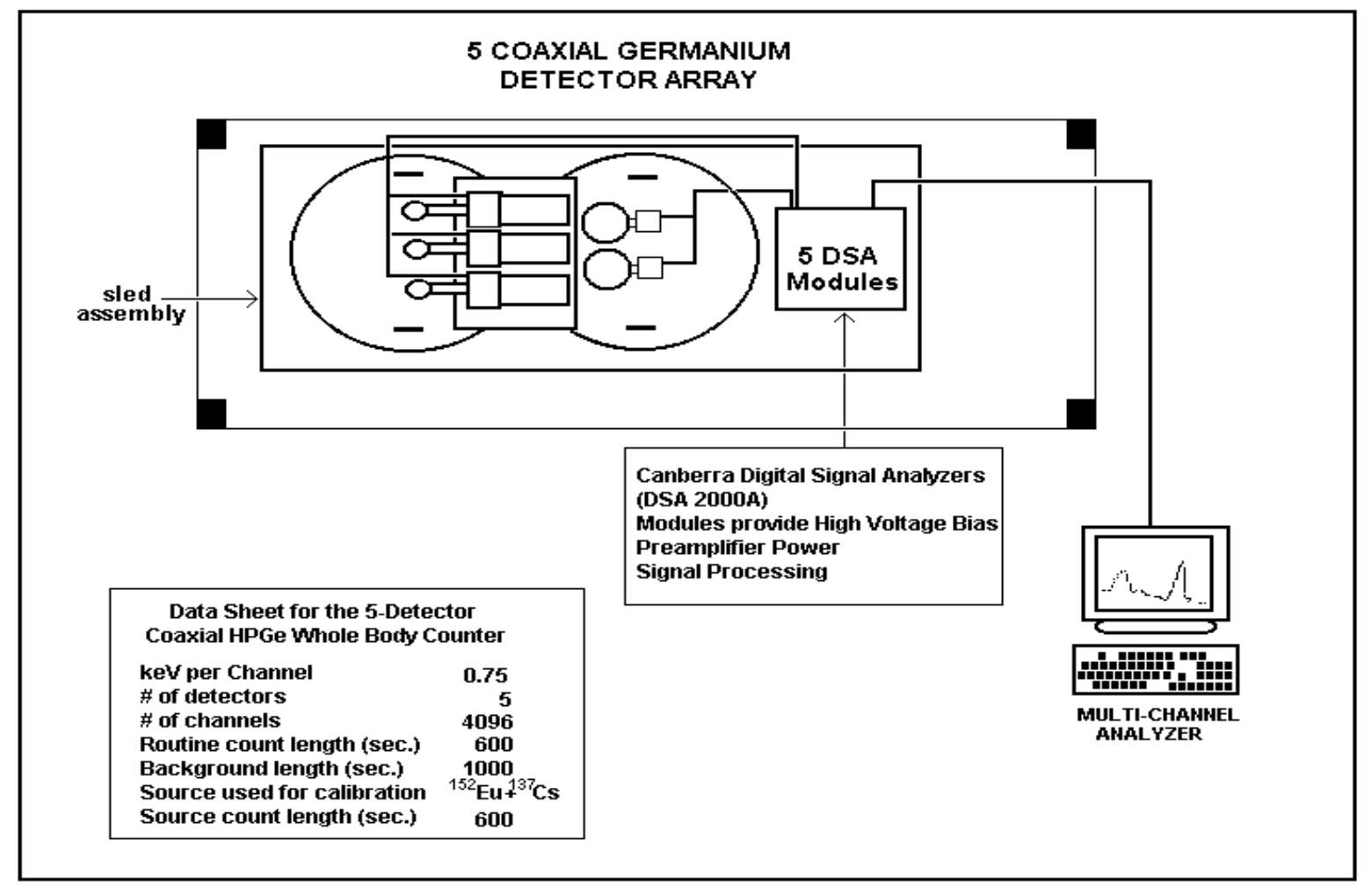

Figure 2.6. Semiconductor Detector Signal Processing Diagram

The N-type planar germanium detectors used for routine lung counting have germanium crystals with a $38-\mathrm{cm}^{2}$ active area and are 20-30 mm thick (see Figure 2.7). The planar HPGe crystal is made of n-type material with an implanted outer $\mathrm{P}^{+}$contact layer, typically 0.3 microns thick, and a diffused center contact. A $2000 \mathrm{~V}$ to $3000 \mathrm{~V}$ negative bias is usually applied to the crystal for the older detectors from Princeton Gamma Tech, Inc.. For the Canberra Industries' detectors, the bias applied to the crystal is typically a positive $3500 \mathrm{~V}$. The entrance windows are either $0.76-\mathrm{mm}(0.03$-in.) thick beryllium or 0.6$\mathrm{mm}$ thick carbon composite material with a $38.5-\mathrm{cm}^{2}$ area. The output charge from absorption of a photon in the crystal is amplified to a measurable electrical signal by the first stage of the charge sensitive preamplifier. The resistive-feedback preamplifiers are integral with the dewar and cryostat configuration. The preamplifiers have a 100-microsecond decay time with an energy rate product of 50,000 MeV/s which covers the range of expected count rates typically encountered with in vivo monitoring. 


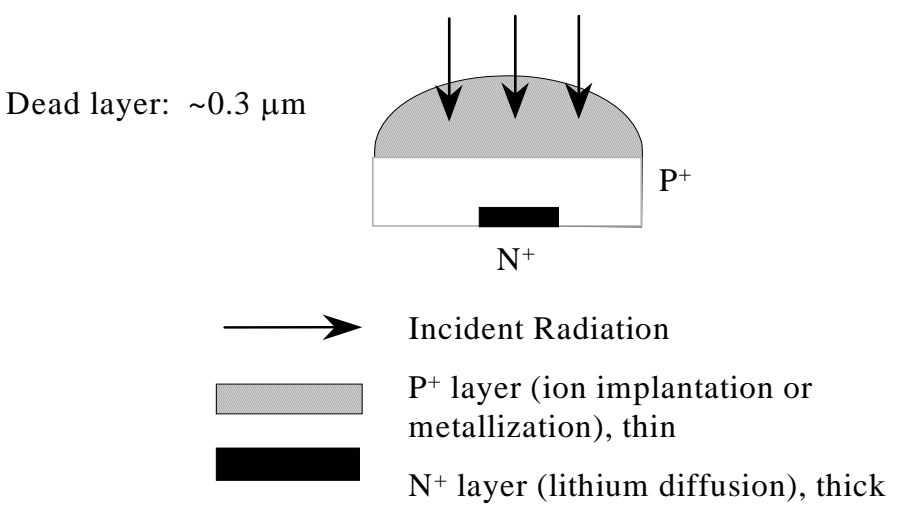

Figure 2.7. Schematic of N-Type Planar HPGe

The coaxial HPGe detectors range from $120 \%$ to $130 \%$ in efficiency relative to a 3-x-3-in. sodium iodide detector. There are two styles of coaxial detectors in use. The GEM series detectors manufactured by Ortec, Inc. contain P-type HPGe crystals and operate at a positive bias in the range of $2500 \mathrm{~V}$ to 5000 V. The crystals are configured with a 700-micron thick Li-diffused outer layer and a 1-mm (0.04-in.) thick aluminum absorbing layer (Figure 2.8). The crystal is housed in a nickel-plated magnesium end cap. The typical size of these HPGe detectors is 82-mm (3.3-in.) in diameter and 103-mm (4.1-in.) in length. The crystals and first stage electronics are configured in horizontal cryostats.

The second style of coaxial detector is configured in a vertical cryostat orientation. These detectors from Canberra Industries have aluminum end caps and the crystal is cylindrical with an n-type contact on the outer surface and a p-type contact on the surface of the axial well. Typical crystal dimensions are 85mm diameter by $90-\mathrm{mm}$ in length. The relative efficiency of these type detectors is $126 \%$ to $129 \%$.

The electron-hole pairs formed by the ionizations produced from the absorption of photon energies in the germanium are swept apart and give rise to a pulse in the load resistor. The pulse is amplified in the first stage of the charge sensitive preamplifier and the pulse subsequently processed with the integrated digital signal processing components.

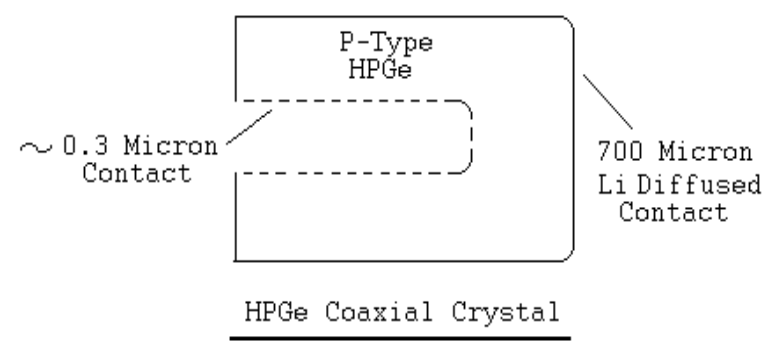

Figure 2.8. Schematic of a Typical P-Type Coaxial HPGe Crystal

\subsubsection{Other Detector Types}

A cadmium telluride (CDT) detector is available for measuring wounds with large amounts of activity. The CDT detector would be used to help localize the radioactive material in the wound.

\subsubsection{Detector Maintenance and Repair}




\subsubsection{Semiconductor Detectors}

Onsite maintenance of semiconductor detectors is performed at the IVRRF. The instrument technician has trained at the manufacturer's facilities in detector repair. In many cases, the planar and coaxial HPGe detectors can be repaired onsite, saving time and money. Only infrequently do detectors have to be sent back to the manufacturer for repairs. When internal components are exposed, work may be done in a dry nitrogen gas environment to minimize contaminants introduced into the vacuum vessel.

The repairs discussed below require breaching the vacuum vessel and subsequently the vacuum vessel must be evacuated before the HPGe detector can be used.

- Field Effect Transistor (FET) replacement-When it fails, the FET is replaced with the same or an equivalent FET (e.g., Interfet 2N4493 for the 38- $\mathrm{cm}^{2}$ HPGe detectors), often improving detector resolution and reducing the electronic noise. Selected FETs are identified to minimize noise when they are cooled to liquid nitrogen temperatures.

- Cryostat repair-The loss of vacuum integrity degrades resolution and the performance of the detector. A Varian VS Series Helium Mass Spectrometer Leak Detector is used to determine the location of the leak. Penetration points of the cryostat are the most likely locations to leak. The repair of leaks can consist of replacing o-ring seals, preamplifier electrical feed-throughs, and entrance windows. After repairing a leak, thermal cycling a detector between 140 to $200^{\circ} \mathrm{F}$ for 24 hours is typically done while evacuating the vacuum vessel. Detectors may also be thermally cycled to room temperature.

- Detector window repair and replacement-The thin detector entrance windows on the planar HPGe detectors can be repaired or replaced if they are damaged or leaking. Small leaks may be repaired using an epoxy adhesive. Removal of a damaged window requires heating of the epoxy to remove it. Residual epoxy must be removed from the surfaces where the window seals to the end cap to achieve a good vacuum seal after repairs are complete.

- Feedback resistor replacement-These resistors are very fragile, and care must be taken when installing them to avoid damage. A 50 -gigaohm resistor is typically used for a $38-\mathrm{cm}^{2}$ planar HPGe.

\subsubsection{Scintillation Detectors}

The NaI(Tl) scintillation detectors operate at room temperature in a light tight enclosure and usually require only minimal maintenance. This maintenance includes periodically balancing the photomultiplier tubes (PMTs) and replacing the optical coupling compound between the PMTs and the crystal as needed. Deterioration of the detector resolution can result from the imbalance of PMTs and degradation of the coupling compound. Balancing the PMTs in the scintillation detector requires a gamma-emitting source to collect a spectrum from the detector using only one of the PMTs at a time. The gain for each PMT is adjusted until the peak channel locations are the same for all detectors in the array.

Should a detector be damaged, the $\mathrm{NaI}(\mathrm{Tl})$ crystal itself cannot be repaired onsite and must be returned to the vendor. The PMTs are replaced if they fail.

\subsubsection{Determination of Detector Resolution}

The energy resolution is one indicator of a radiation detector's performance capability. A detector's resolution will determine to what extent a measurement with the detector is able to resolve closely spaced 
photopeaks in the spectrum. It is typically expressed as a percentage of the reference peak energy at the full width at half maximum (FWHM) value of the pulse height distribution at the particular photon energy. The ANSI/IEEE Standard 325-1986, IEEE Standard Test Procedures for Germanium GammaRay Detectors, contains test protocols for energy spectroscopy measurements. Several different radionuclides such as ${ }^{152} \mathrm{Eu},{ }^{241} \mathrm{Am},{ }^{137} \mathrm{Cs}$, and ${ }^{60} \mathrm{Co}$ may be used for energy resolution measurements depending on the particular application. The resolution is a measure of the detector's ability to accurately place full-energy peak pulses at a consistent voltage level. For the same amount of deposited energy, a narrow, tall full-energy peak has less fluctuation among pulses compared to a shorter wider peak and can better differentiate closely spaced peaks. The resolution is determined using Equation (2.1) and is illustrated in Figure 2.9. Resolution values for the large $\mathrm{NaI}(\mathrm{Tl})$ detectors are typically in the range of 8$10 \%$ at $662 \mathrm{keV}$. It is common practice to express the resolution of a NaI detector as a percentage of the peak energy. For solid state detectors, the resolution is expressed in terms of $\mathrm{eV}$ or $\mathrm{keV}$.

$$
\% \text { Resolution }=\frac{(\text { Channel } C(\text { counts })-\text { Channel } A(\text { counts })) \times 100}{\text { Channel } B(\text { counts })}
$$

Daily resolution checks are performed on all detectors used for routine measurements. Typical resolution for the $120-130 \%$ coaxial HPGe detectors is $2.5 \mathrm{keV}$ at $1408 \mathrm{keV}$ and $1.4 \mathrm{keV}$ at $122 \mathrm{keV}$. At $59.5 \mathrm{keV}$, the resolution of the $38-\mathrm{cm}^{2}$ planar HPGe detectors is typically $600-800 \mathrm{eV}$.

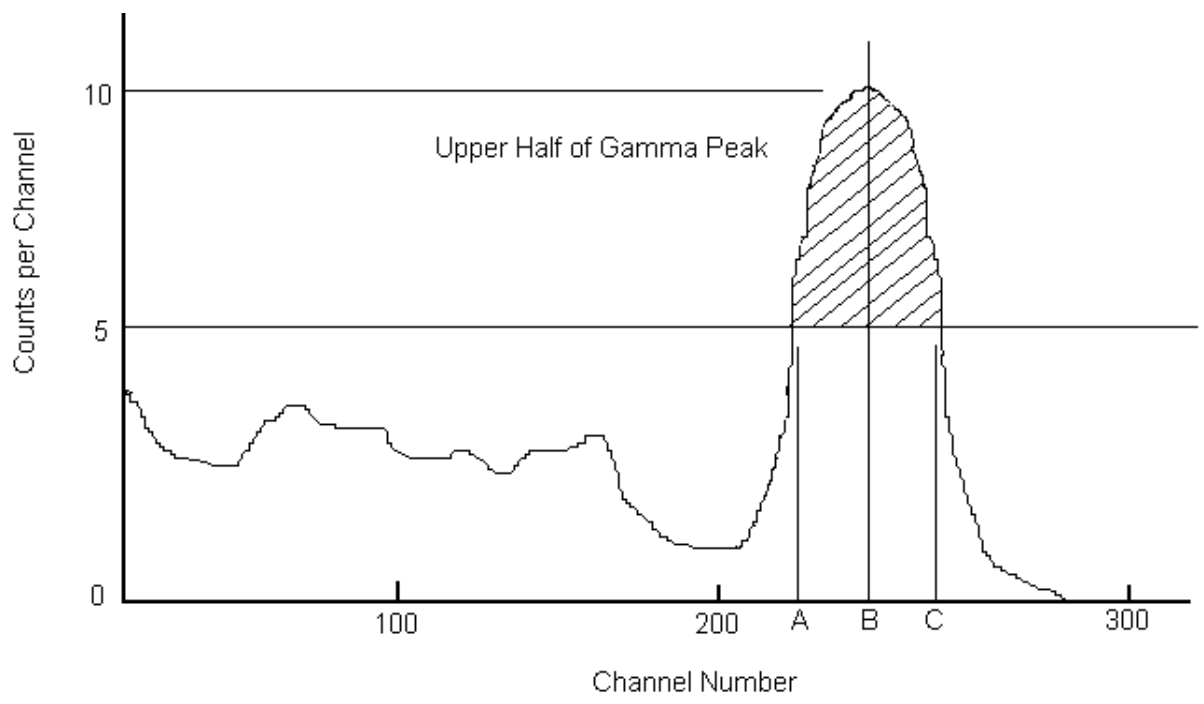

Figure 2.9. Illustration of Detector Resolution Calculation Parameters

\subsubsection{Diagnosis of Detector Problems}

A routine surveillance program is implemented to monitor detector performance. Degradation in a detector's performance can be caused by electronic instabilities, noise, microphonics, ground loops, electronic component failure, vacuum vessel leaks, etc. Daily efficiency and background checks of the detection systems are performed and trend analyses are performed quarterly. The energy centroids and resolution for the individual detector spectra and the summed spectrum for each system are also checked daily. If a system's centroid or resolution value is consistently outside the established limits and/or adversely affects the system efficiency, the cause is investigated and the condition corrected. In addition, the background count rates in selected regions of interest (ROI) are measured each day and trends are analyzed quarterly. 
The performance of electronic components is also routinely monitored. The output from the amplifiers, preamplifiers and digital processors is periodically checked for pulse shape and amplitude with an oscilloscope. The low-energy portion of the spectrum is routinely inspected for noise signals. The stability of the output from high voltage power supplies and the stability of the nuclear instrument module (NIM) bin voltages are also periodically measured.

Noise in a spectrum is generally defined as the extraneous events counted near the zero-energy end of the spectrum although it can appear at higher energies. In scintillation detectors, it is mostly a result of spontaneous emission from the photocathode of the PMT or from a light leak .

There is inevitably noise at low energies (generally less than $100 \mathrm{keV}$ ) for the large NaI detectors with multiple PMT. The baseline noise signal is monitored periodically so adjustments can be made as needed. For the planar HPGe detectors, the diode leakage current induces electronic noise in the 0 to 10 $\mathrm{keV}$ range and usually does not interfere with counting unless it is large enough to affect deadtime. These style germanium detectors are more sensitive to microphonic induced noise signals compared to the coaxial germanium detectors.

Noise can also appear as discrete peaks at high energies. If unidentified peaks appear in a spectrum that has a peak-width narrower than a gamma peak of equal energy, it can result from a malfunction of an electronic component or could be a result of microphonic signals. If the peak is the same width as a gamma ray line, unshielded sources may be present. Review of measurement results and peak search results is the primary tool for identifying extraneous peaks in spectra.

\subsection{Data Acquisition and Processing Equipment}

Energy deposited in the NaI and HPGe detectors is converted to a voltage pulse, shaped, amplified, digitized, and then stored in the multichannel analyzer (MCA). Each channel of the MCA corresponds to an energy defined by the energy calibration equation. After the spectrum is acquired in the MCA it is transferred to memory. A peak search algorithm is used to identify peaks and to quantify their relevant properties. The standard Abacos peak search program is used for routine counting. The standard peak search program evaluates the entire spectrum between specified start and end channels. A library-driven peak search is also available.

Control of the counting systems is done from a central computer system. The Model DS-10 Alpha Workstation (Alphastation) manufactured by HP/Compaq Corporation is the primary computer. The Alphastation includes 1.0 Gb RAM, two 18-Gb hard disks, an 18-inch monitor, 600-Mb CD-ROM (compact disk read-only media), and a DAT tape drive used with 4-mm tapes each with 20/40-Gb capacity. The system operates with the Open VMS operating system. The Alphastation is interfaced via Ethernet cabling to several acquisition interface modules (AIM) and digital signal analyzers that are used to acquire spectra from each of the counting systems. Several personal computers are networked to the Alphastation and communicate using terminal emulation software.

The application software is the AbacosPlus System from Canberra Industries. Software control of counting systems is done using the Genie-ESP application that is bundled with the Abacos Plus. The Abacos Plus software is used to set up the MCA, calibrate counters, acquire spectra, analyze spectra, analyze quality assurance (QA) data, and store, retrieve, and transfer data. A series of command procedures and programs were developed to customize the software to handle Hanford-specific information and to accommodate simultaneous operation of several counting systems using a combination of menu driven and graphical user interfaces. Detailed information about how the Abacos Plus system 
acquires, analyzes, and stores spectra is contained in the various users' manuals and correspondence from the vendor.

\subsection{Signal Processing Electronics}

This section describes the general operation of the components of the signal processing electronics.

\subsubsection{Preamplifiers}

The output from a detector is a small charge pulse. It is necessary to precondition the output so that it can be used in a linear amplifier system. The linear amplifier system in turn provides the necessary pulseshaping and amplification required for use with the measuring unit. A preamplifier is generally inserted immediately after the detector to provide integration of the charge output from the PMT of a scintillation detector or a semiconductor detector.

The output of the preamplifier is connected to a pulse-shaping linear amplifier. Impedance-matching between the detector output and the pulse-shaping and amplifier stage that follow is provided by the preamplifier. The length of cable from the preamplifier to the detector is minimized to increase the signal-to-noise ratio.

The planar HPGe detectors used for chest counting have integral preamplifiers within the cryostat housing just behind the detector. These are charge-sensitive, resistive feedback preamplifiers. The coaxial HPGe detectors also employ charge-sensitive, resistive feedback preamplifiers. The preamplifier output from these HPGe detectors are input directly to digital processing modules. The outputs from the $\mathrm{NaI}(\mathrm{Tl})$ scintillation detectors PMT used in the standup counter are input to non-inverting scintillation preamplifiers. Further details on the operating specifications are found in the vendor manuals for the specific preamplifiers.

\subsubsection{Amplifiers}

The main linear amplifier further increases the millivolt signal from the preamplifier to a $0.1-10-\mathrm{V}$ signal so that it can be used within the dynamic operating range of the computer and analysis equipment that displays the results. Linear amplifiers are used to ensure that the fixed relationship between the input and output signal is maintained. Only NaI detectors and a couple germanium detectors still use analog amplifiers.

To measure individual signals, independent of previous ones, the signal decay time must be reasonably short compared with the input repetition rate in order to reduce pulse pile-up distortion. In addition, to be proportional to the amplitude of the signal from the preamplifier (and, therefore, the charge from the detector), the duration of the output signals from the amplifier must be longer than the rise time of the input pulse. Further details on the operating instructions and characteristics are found in the instruction manuals for the specific amplifiers.

The amplifier outputs are routed to a mixer/router module and then to an analog to digital converter (ADC). The digital signal is then accumulated in a memory location of the MCA that corresponds to the pulse amplitude.

\subsubsection{Digital Signal Processing}


Integrated signal processing units are used for most of the routine counting systems. The preamplifier from each HPGe detector is connected to a digital signal processing module. These integrated modules perform amplification, digital signal filtering, conversion, digital stabilization, and also function as a multichannel analyzer. The signal is digitized first which minimizes the effects of gain changes and instabilities (e.g., line voltage fluctuations) which can occur when the preamplifier output signal is amplified before being digitized. They also provide a high voltage supply for the detectors. There are many user adjustable parameters (e.g., high voltage, coarse gain, fine gain, super fine gain, etc.) as well as automatic pole-zero adjustment. The units are connected to a local area network and are computer controlled. The spectra are transferred over the network to the central computer, the results calculated, and ultimately the file is stored on disk.

\subsection{Spectroscopy Equipment: Multichannel Analyzer}

The IVMP uses computer-based MCA for acquisition of spectral data. The electronic components described in the previous section provide the proper input signal to the MCA. The MCA is operated in the pulse height analysis mode where the amplitude of the voltage pulses is proportional to the energy absorbed by the detector. The MCA constructs a frequency distribution of the number of occurrences versus the memory address. The memory address is the channel number that is proportional to the input signal voltage. Thus a spectrum of the number of pulses versus voltage level is obtained and is interpreted as the number of counts per energy interval. Typical energy calibrations are $20 \mathrm{keV} / \mathrm{channel}$ over 256 channels for the NaI whole body counting system, $0.75 \mathrm{keV} /$ channel over 4096 channels for the coaxial HPGe whole body counting system, and $0.25 \mathrm{keV} /$ channel over 2048 channels for the low-energy HPGe systems.

\subsection{Calibration Phantoms}

To determine the amount of radioactive material in the body or in a specific organ or body location, the detector system response is calibrated to establish its response to a known amount and type of radioactive material in a phantom. The response is usually expressed in net counts per photon emitted from the body or organ. This is normally done by determining the system's efficiency for measuring the radioactive material distributed in anthropometric calibration phantoms, which are used to simulate the activity distribution in and the radiation interaction characteristics of human tissue. More detail on the system calibrations is given in Section 5.0 of this manual.

Phantoms are typically fabricated from polyurethane compounds or other plastics with the addition of trace amounts of other materials (e.g., $\mathrm{CaCO}_{3}$ ) to properly simulate the radiation interaction properties of tissue. Known amounts of radioactive material are incorporated in the simulated body or organ. Many different types of calibration phantoms have been developed, each with specific uses for in vivo bioassay. The following sections describe the calibration phantoms used by the IVMP staff.

\subsubsection{Bottle-Manikin Absorption Phantom}

The bottle-manikin absorption (BOMAB) phantom consists of 10 polyethylene cylinders of various sizes, which are filled with radioactive solutions or tissue-substitute materials. When properly assembled, the BOMAB phantom approximates the size, weight, and general shape of an adult male and was originally based on the reference man dimensions in ICRP Publication 23. These phantoms can be filled with liquid or solid filler materials. Specifications for different size BOMAB phantoms are found in ANSI N13.35. 
Liquid radioactive solutions are easy to prepare and can be discarded after use to allow the phantom to be reused. The potential for leakage and plateout or precipitation of activity are of concern when using liquid-filled phantoms. For these reasons, most of the BOMAB phantoms used for calibration are filled with solid polyurethane compounds. The polyurethane-based tissue substitutes developed at PNNL for BOMAB phantoms have attenuation coefficients within 2-5\% of human soft tissues. Table 2.1 lists the current inventory and activity content of the BOMAB phantoms.

\begin{tabular}{|c|c|c|c|}
\hline $\begin{array}{l}\text { Phantom ID } \\
\text { No. }\end{array}$ & Isotope & $\begin{array}{c}\text { Activity, nCi (kBq) } \\
\pm 95 \% \text { Confidence Level } \\
\end{array}$ & $\begin{array}{c}\text { Reference } \\
\text { Date }\end{array}$ \\
\hline 418 & ${ }^{40} \mathrm{~K}$ & $939 \pm 110(35 \pm 4)$ & $4 / 27 / 90$ \\
\hline 416 & ${ }^{137} \mathrm{Cs}$ & $307 \pm 8(11.4 \pm 0.3)$ & $1 / 12 / 90$ \\
\hline 423 & ${ }^{133} \mathrm{Ba}$ & $\begin{array}{c}289.6 \pm 2.8(10.72 \pm 0.10) \\
120 \pm 5 \%(4.4)\end{array}$ & 9/23/92 \\
\hline 417 & $\begin{array}{l}{ }^{125} \mathrm{Sb} \\
{ }^{154} \mathrm{Eu} \\
{ }^{155} \mathrm{Eu}\end{array}$ & $\begin{array}{c}240 \pm 1.3 \%(8.8) \\
960 \pm 0.5 \%(35) \\
240 \pm 0.8 \%(8.3)\end{array}$ & 1/1/95 \\
\hline 431 & ${ }^{40} \mathrm{Eu}$ & $\begin{array}{c}224.0 \pm 1.4(8.288 \pm 0.052) \\
115 \pm 5 \%(4.3)\end{array}$ & 6/17/93 \\
\hline 432 & ${ }^{137} \mathrm{Cs}$ & $\begin{array}{c}503.7 \pm 8.5(18.64 \pm 0.31) \\
120 \pm 6(4.44 \pm 0.22)\end{array}$ & 3/12/92 \\
\hline 507 & $\begin{array}{l}{ }^{137} \mathrm{Cs} \\
{ }^{109} \mathrm{Cd} \\
{ }^{60} \mathrm{Co}\end{array}$ & $\begin{array}{c}60 \pm 3^{(\mathrm{a})}(2.2) \\
1850 \pm 90^{(\mathrm{a})}(68) \\
87 \pm 4^{(\mathrm{a})}(3.2)\end{array}$ & $4 / 1 / 02$ \\
\hline
\end{tabular}

${ }^{\text {(a) }}$ Uncertainty values at $99 \%$ confidence level

Table 2.1. BOMAB Phantom Inventory

The BOMAB phantom is used primarily for the calibration of whole body counting systems used for measurements of fission and activation product radionuclides in the body. Two sizes of BOMAB phantoms are used to provide calibrations appropriate for the size range of workers at Hanford. One is based on the Reference Man dimensions in ICRP Publication 23. A second phantom weighing 230 lbs. at a height of $194 \mathrm{~cm}$ is used for workers weighing $\geq 200 \mathrm{lbs}$. Both phantoms are consistent with the specifications in ANSI N13.35.

Examples of counting systems that use this type of phantom are the standup counter, the coaxial HPGe scanning system, and the shadow-shield system in the mobile trailer.

Figure 2.10 depicts a Reference Man BOMAB phantom in the typical configuration used for calibrations. The nominal size and weights of the Reference BOMAB phantom components are listed in Table 2.2. 


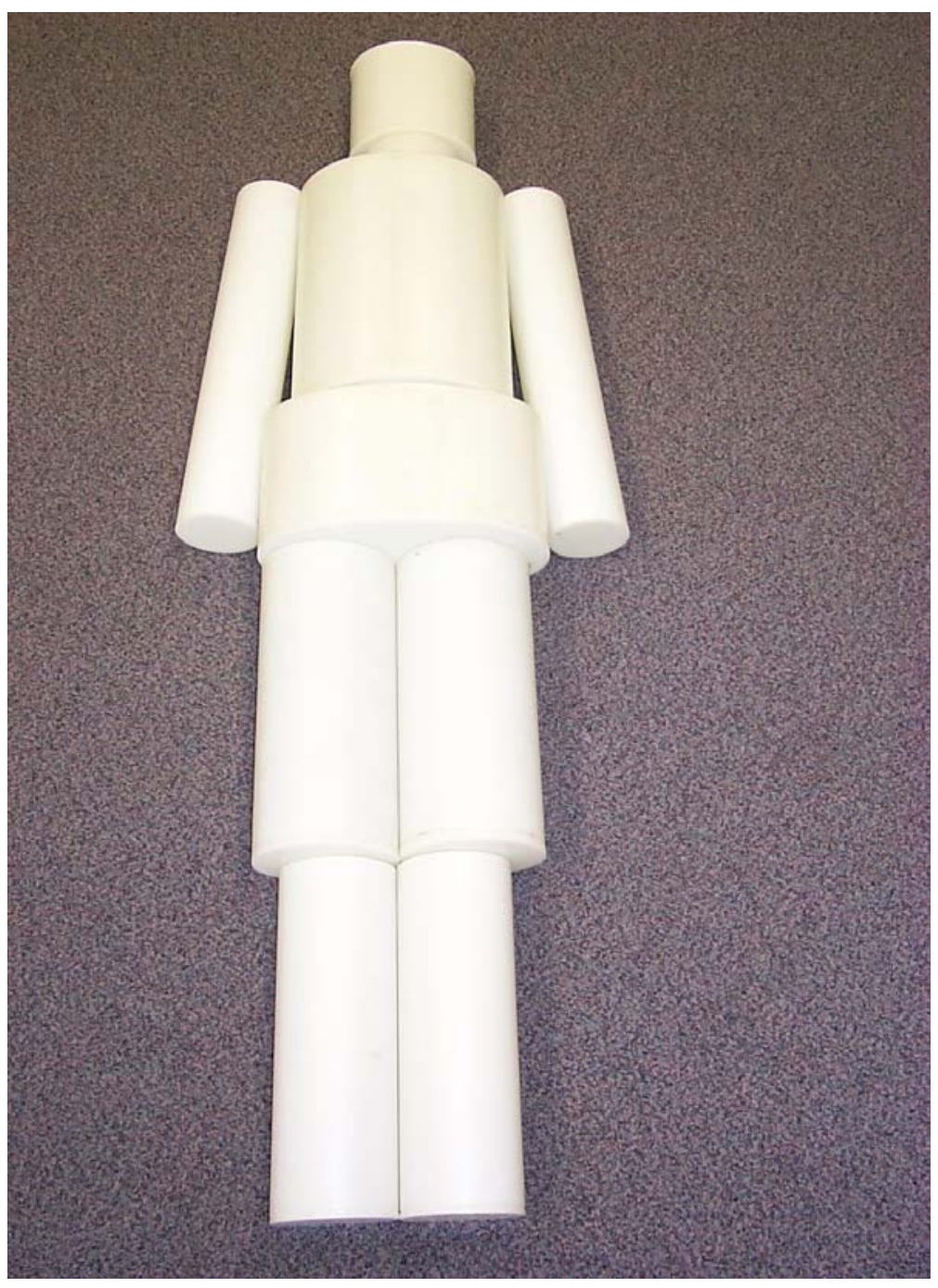

Figure 2.10. BOMAB Calibration Phantom 
Table 2.2. Typical Weights and Volumes of the Reference BOMAB Phantom

\begin{tabular}{lrr}
\hline $\begin{array}{c}\text { Phantom } \\
\text { Part }\end{array}$ & $\begin{array}{c}\text { Fill Vol. } \\
\text { ml }\end{array}$ & $\begin{array}{c}\text { Total } \\
\text { Weight, } \mathbf{g}\end{array}$ \\
\hline Head & 3,490 & 4,200 \\
Neck & 1020 & 1,135 \\
Torso & 16,880 & 18,500 \\
Arms & 7,500 & 9,990 \\
Pelvis & 9,930 & 11,690 \\
Thighs & 12,000 & 14,980 \\
Lower legs & $\underline{7,410}$ & $\underline{10,215}$ \\
Total & 58,240 & 70,710 \\
\hline
\end{tabular}

\subsubsection{Tissue-Equivalent Torso Phantoms}

A torso phantom made from a tissue-substitute polyurethane compound is shown in Figure 2.11. This style of phantom was specifically constructed for calibration of in vivo measurement systems used to detect transuranic radionuclides in the body and in particular the lungs, liver, and lymph nodes. Developed at the Lawrence Livermore National Laboratory (LLNL), the phantom is made of polyurethane with different concentrations of calcium carbonate added to simulate the radiation interaction characteristics of lean muscle, adipose-muscle mixtures, cartilage, and bone. The phantom is designed for the insertion of organ inserts such as lungs and livers containing the radioactive material. The radioactive materials homogeneously distributed in the organ inserts which are also made of polyurethane with the appropriate density to simulate lung tissue. One of the torso phantoms was among the original series of 16 developed at LLNL and two are commercially manufactured phantoms. Griffith et al. (1978) discuss the phantom design in greater detail. A history of the development of the torso phantom is also available (Taylor 1997). Further background information on the torso phantom and newer laser scan data is also available (Snyder 2010). 


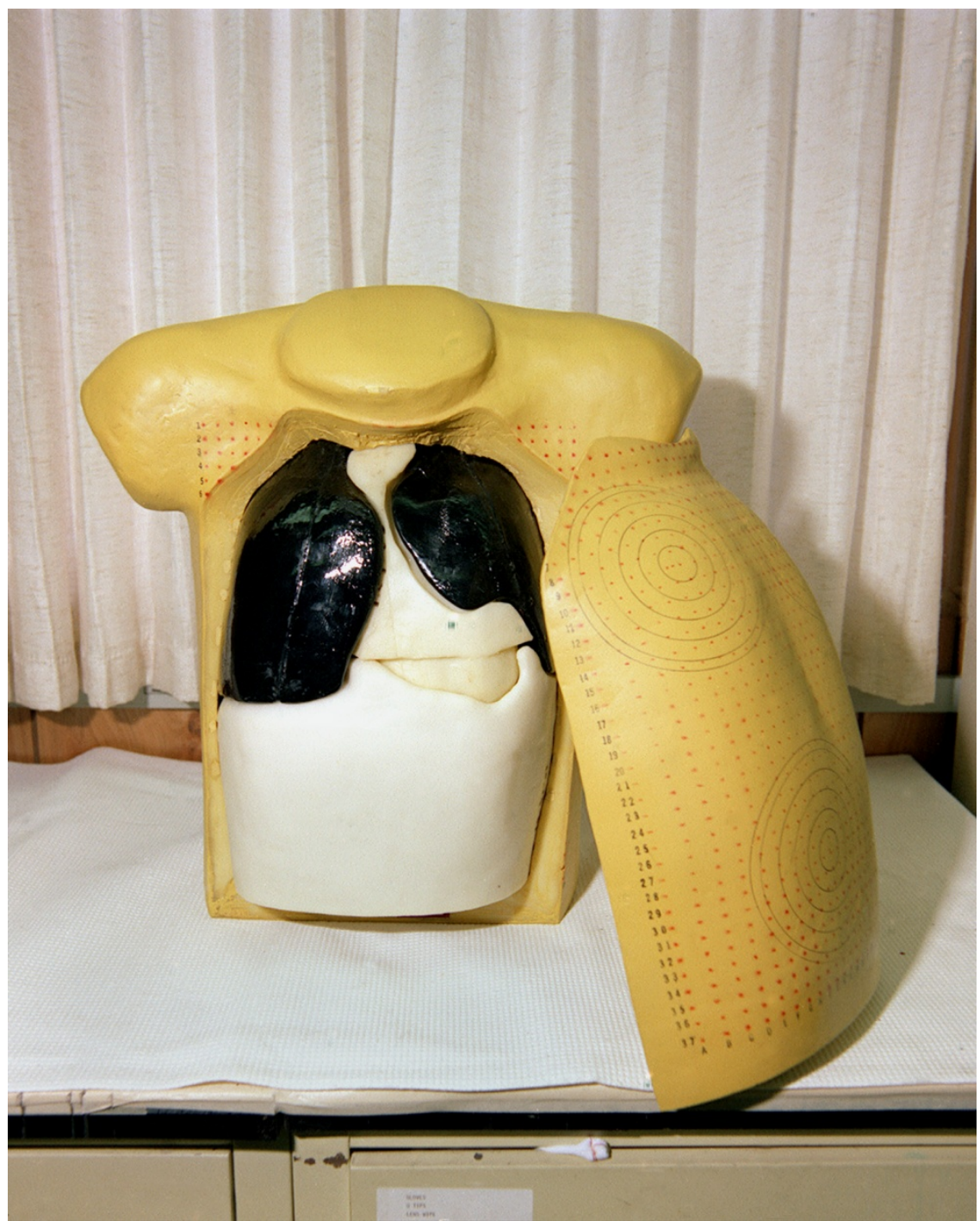

Figure 2.11. Tissue-Equivalent Torso Phantom

The LLNL torso phantom contains a rib cage, sternum and clavicles. These skeletal components are made of artificial bone-substitute material. The phantom does not contain simulated scapulae or the spine, which limits its use for posterior lung counting especially for low-energy photons. Tissuesubstitute chest overlays are used with the torso phantom to simulate a range of worker chest-wall thicknesses and fat compositions. There are three sets of chest overlays available: one set of $50 \%$ fat and $50 \%$ muscle-equivalent plastic; a second set of $87 \%$ fat and $13 \%$ muscle-equivalent plastic; and $100 \%$ muscle equivalent overlays There are four overlays in each set. The $87 \%$ fat and $13 \%$ muscle overlays have historically been used for calibration purposes based on the assumption that additional chest thickness in workers is more likely to be fat than muscle. The muscle to fat ratio of the chest wall is not critical for measurements at $59.5 \mathrm{keV}$ and above. There is only a small ( 2\%) difference in transmission through $100 \%$ muscle and $87 \%$ fat for a $59.5 \mathrm{keV}$ photon. The tissue composition becomes much more critical when directly measuring the $\mathrm{L} x$-rays from plutonium and may require more in-depth evaluation of the person's muscle to fat ratio. 
The LLNL torso phantom provides adequate representation of the attenuation qualities of the human body for the L x-rays from plutonium. A study compared 18 humans who inhaled known quantities of

${ }^{92 \mathrm{~m}} \mathrm{Nb}$ (Gunston and Jeffries 1986; Newton et al. 1985). The study originated in England and was funded by the International Atomic Energy Agency (IAEA). The IVRRF participated in the measurements of these subjects, with good agreement between the phantom calibrations and the ${ }^{92 \mathrm{~m}} \mathrm{Nb}$ results. The mean observed efficiency in the subjects was within $20 \%$ of the predicted efficiency based on the torso phantom measurements of the $15.8 \mathrm{keV}$ to $17.7 \mathrm{keV}$ x-rays from ${ }^{92 \mathrm{~m}} \mathrm{Nb}$ in the lung. The measurements were performed with phoswich detectors and the results have been extrapolated to indicate the expected performance for arrays of HPGe detectors.

An assortment of lung phantoms with different radionuclide loadings are maintained at the IVRRF for use in calibrating the detector systems. Table 2.3 contains a listing of lung and liver inserts for the torso phantoms.

\subsubsection{Fission Product Phantom}

A whole body phantom sold commercially as the Fission Product (FP) phantom ${ }^{1}$ is a modification of the tissue-equivalent torso phantom, having additional body parts incorporated to complete the entire physique of a "reference" individual. A pelvis, head, neck, arms and legs were added in order to extend the use of the torso phantom to various whole body and organ measurement procedures (see Figure 2.12). The limbs of the FP phantom are articulated, and the phantom has a set of four chest overlays composed of $100 \%$ International Commission on Radiological Units and Measurements (ICRU) muscle-equivalent tissue substitute.

${ }^{1}$ Radiology Support Devices, 1904 East Dominguez Street, Long Beach, CA, 90810. 
Table 2.3. Torso Phantom Organ Inventory

\begin{tabular}{|c|c|c|c|c|}
\hline \multirow[b]{2}{*}{ Radionuclide } & \multirow{2}{*}{$\begin{array}{c}\text { Phantom } \\
\text { ID No. }\end{array}$} & \multirow{2}{*}{$\begin{array}{c}\text { Calibration } \\
\text { Date }\end{array}$} & \multicolumn{2}{|c|}{ Total Activity $\pm 95 \%$ C.L. ${ }^{(a)}$} \\
\hline & & & $\mathrm{nCi}$ & $\mathbf{k B q}$ \\
\hline \multicolumn{5}{|c|}{ Lung } \\
\hline${ }^{238} \mathrm{Pu}$ & LG-200/526 & $2 / 26 / 81$ & $2250 \pm 170$ & $83.25 \pm 6.29$ \\
\hline${ }^{239} \mathrm{Pu}$ & LG-207/513 & 3/13/81 & $6300 \pm 310$ & $239.7 \pm 11.5$ \\
\hline${ }^{241} \mathrm{Am}$ & LG-202/516 & $3 / 4 / 81$ & $620 \pm 46$ & $22.9 \pm 1.7$ \\
\hline${ }^{241} \mathrm{Am}$ & LG-214 & $8 / 3 / 88$ & $20 \pm 2$ & $0.74 \pm 0.07$ \\
\hline${ }^{235} \mathrm{U}(93 \%)$ & LG-206/550 & $4 / 6 / 82$ & $204 \pm 14$ & $102 \pm 7.0 \mathrm{mg}$ \\
\hline${ }^{n a t} \mathrm{U}$ & LG-210/557 & $4 / 11 / 84$ & $103.5 \pm 10$ & $313 \pm 30 \mathrm{mg}$ \\
\hline${ }^{232} \mathrm{Th}$ & LG-201/538 & $4 / 20 / 82$ & $57 \pm 5$ & $521 \pm 43 \mathrm{mg}$ \\
\hline${ }^{60} \mathrm{Co}$ & LG-354 & 10/01/82 & $67.2 \pm 9.3$ & $2.49 \pm 0.34$ \\
\hline${ }^{241} \mathrm{Am}$ & LG-503(Am-008) $)^{(\mathrm{b})}$ & $9 / 21 / 01$ & $545 \pm 2$ & $2.0 \pm 0.07$ \\
\hline${ }^{241} \mathrm{Am}$ & $\mathrm{LG}-468^{(\mathrm{b})}$ & 7/7/98 & $396 \pm 11$ & $15 \pm 0.6$ \\
\hline${ }^{152} \mathrm{Eu}$ & & 7/7/98 & $404 \pm 17$ & \\
\hline${ }^{241} \mathrm{Am}$ & LG-495 ${ }^{(\mathrm{b})}$ & $3 / 10 / 00$ & $59 \pm 0.2$ & $2 \pm 0.01$ \\
\hline${ }^{152} \mathrm{Eu}$ & & $3 / 10 / 00$ & $59 \pm 0.5$ & $2 \pm 00.2$ \\
\hline \multicolumn{5}{|c|}{ Liver } \\
\hline${ }^{238} \mathrm{Pu}$ & LIPU8-204/502 & 2/26/81 & $4680 \pm 220$ & $173 \pm 8$ \\
\hline${ }^{239} \mathrm{Pu}$ & LIPU9-205/507 & $3 / 13 / 81$ & $13.63 \pm 0.65 \mu \mathrm{Ci}$ & $504.3 \pm 24.1$ \\
\hline${ }^{241} \mathrm{Am}$ & LIAM-203 & $2 / 27 / 81$ & $1530 \pm 43$ & $56.61 \pm 1.59$ \\
\hline${ }^{\text {nat }}$ Th & LITH-211/541 & N/A & $63.9 \pm 3$ & $586 \pm 29 \mathrm{mg}$ \\
\hline${ }^{152} \mathrm{Eu}^{88}{ }^{88} \mathrm{Y}$ & LIYEU-001 & 6/17/93 & $7.17 \pm 0.08(\mathrm{Eu})$ & $0.265 \pm 0.003$ \\
\hline & & & $15.2 \pm 0.5(\mathrm{Y})$ & $0.562 \pm 0.017$ \\
\hline
\end{tabular}

(a) Total activity uncertainty value at $95 \%$ confidence level.

(b) Total activity uncertainty value at $99.7 \%$ confidence level. 


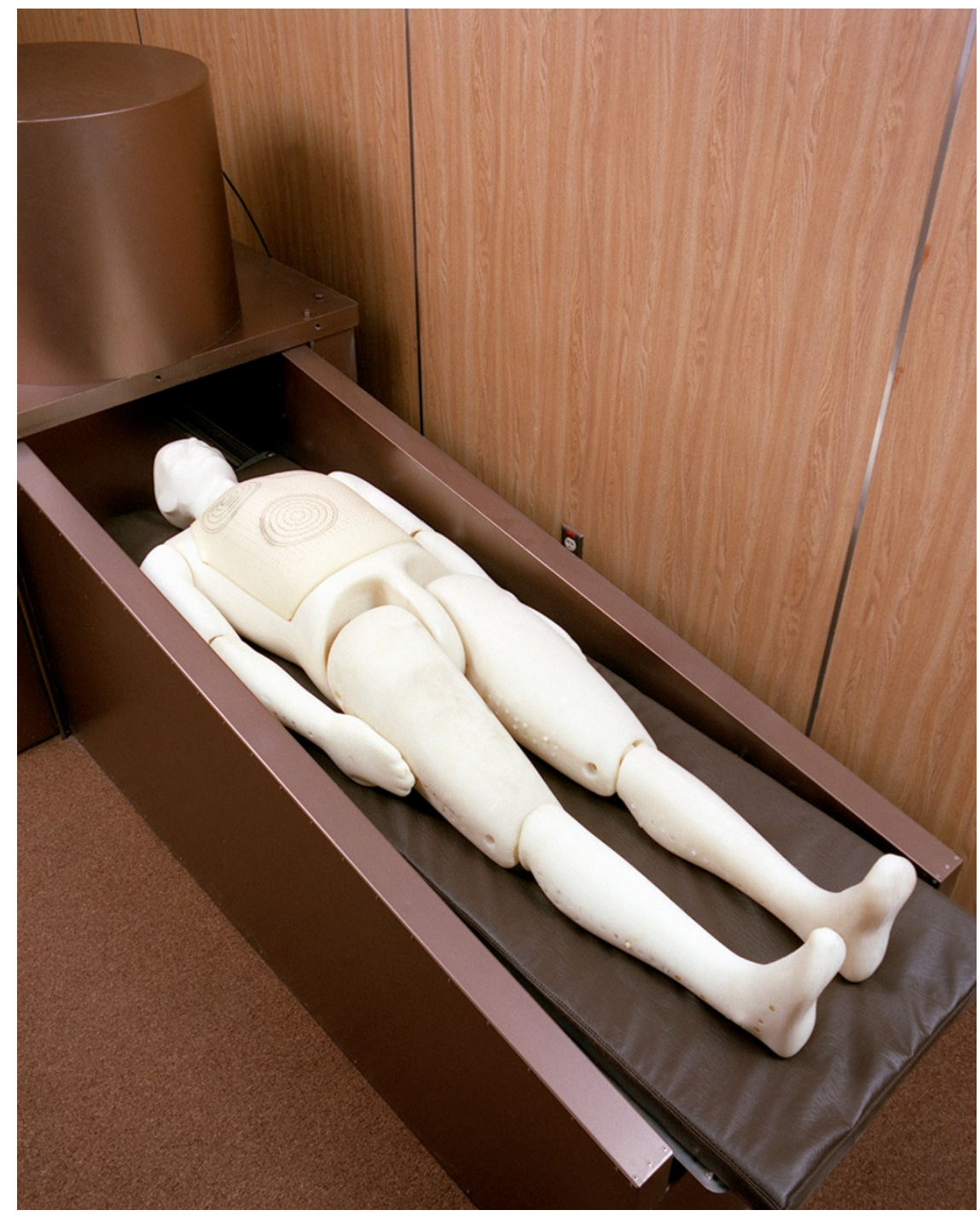

Figure 2.12. Fission-Product Calibration Phantom

The torso section of the FP phantom is used primarily for posterior chest-counting calibrations because unlike the other torso phantoms, the FP phantom includes bone substitute scapulae. It can also be used for anterior lung counting calibrations and the lung sets listed in Table 2.3 are compatible with the phantom. It is not used for calibration of whole body counting systems.

\subsubsection{Skeletal Phantoms}

Several phantoms were developed with DOE funds to calibrate detectors for quantifying the amount of ${ }^{241} \mathrm{Am}$ in the skeleton and to correct other organ measurements for interferences from skeletal activity. Head, torso, arm, and leg phantoms were made from approximately one-half of a skeleton from a total body donation to the U.S. Transuranium and Uranium Registries (USTUR). The results from the analyses of the bones are described in a 1985 special addition of the Health Physics Journal (McInroy et al, 1985). Figure 2.13 depicts this skeletal phantom. 
There was an estimated $119 \mathrm{nCi}$ of ${ }^{241} \mathrm{Am}$ in the skeleton at the time of death. The level of activity was determined by radiochemically measuring the activity in approximately half of the skeleton and extrapolating to a total skeletal activity based on the ratios of wet weights of the bones. The other half of the skeleton was incorporated into the phantoms mentioned above. The ${ }^{241}$ Am distribution in this skeleton reflects 25 years of bone-remodeling following the intake. The phantom is on loan from the USTUR and is made available through the DOE Phantom Library for use by other DOE and government laboratories.

The head phantom contains half of the skull from the donated body (Hickman et al. 1988). The right half of the skull that contains the activity has been matched with a left half of a skull containing no ${ }^{241} \mathrm{Am}$ activity. The cavities of the composite skull were filled with tissue-equivalent material that simulates the brain, soft tissues, and the skin. For calibration with two detectors over the forehead, the detectors must be placed symmetrically on the head phantom. The positions are then reversed and the count repeated to account for different detector efficiencies. The summation of the two counts is then used to calculate the calibration factor.

The arm (Kephart, 1987) and leg phantoms contain bones from the left arm and left leg encased in tissue-substitute plastic that simulates lean muscle. The phantoms are used to establish ${ }^{241} \mathrm{Am}$ calibration factors for measurements over the wrist, elbows, knees, and ankles. They can also be used to help estimate the contribution from skeletal activity to lung and liver measurements.

The torso phantom contains the right ribs, right scapula, right clavicle, and even numbered vertebrae from the USTUR donor. These are matched with matching bones containing no ${ }^{241} \mathrm{Am}$ to create a symmetrical thoracic skeleton. It does not include the sternum which was radiochemically analyzed. The phantom is used primarily to estimate the contribution from ${ }^{241} \mathrm{Am}$ activity in the skeleton to the measured count rate from a chest or liver measurement.

The leg phantom incorporates the left leg from Case 102 within tissue-substitute polyurethane formed to simulate the soft tissue features of a leg. 


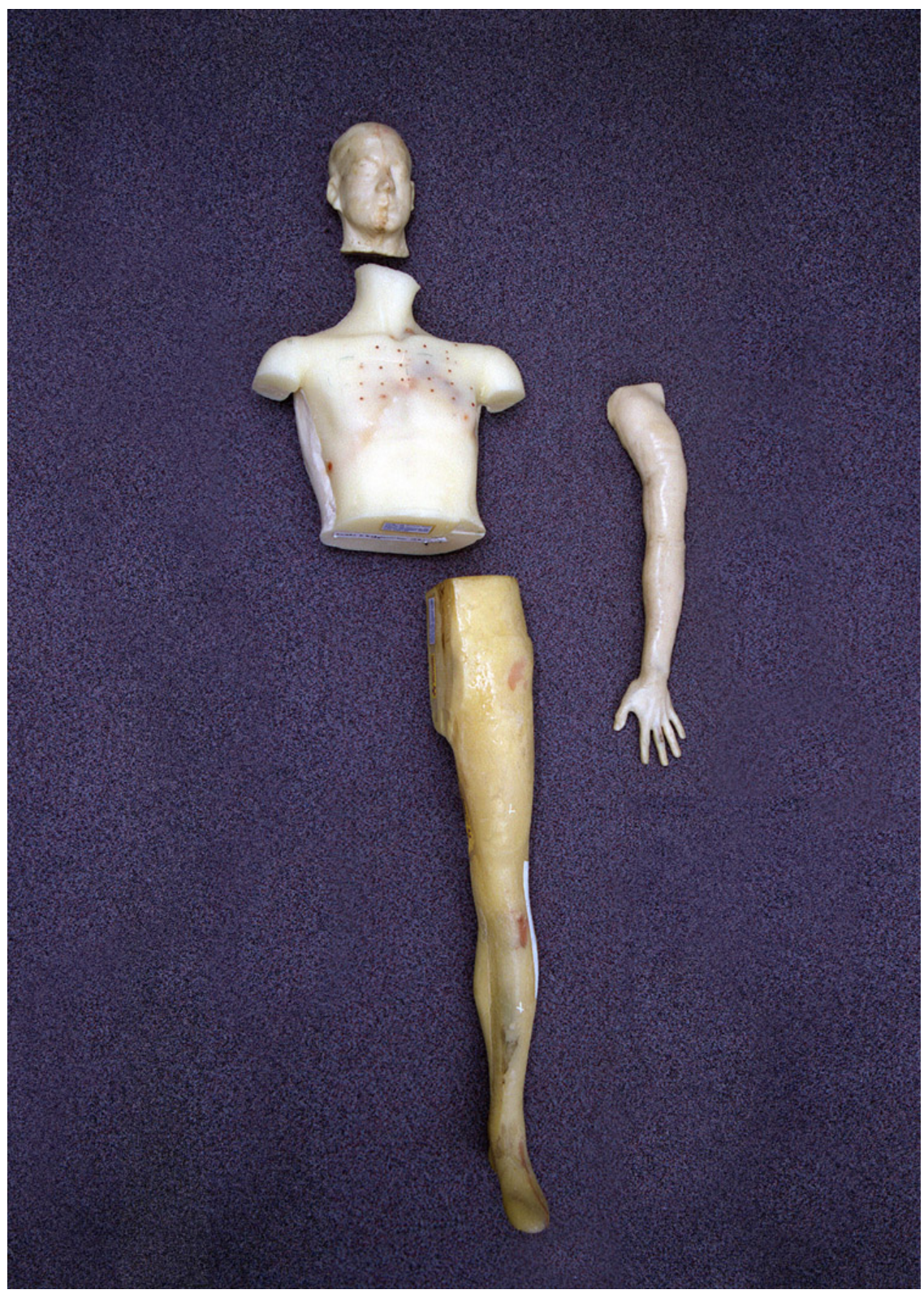

Figure 2.13. Skeletal Phantom

\subsubsection{Thyroid Phantoms}

The head and neck phantom that is used for both ${ }^{125} \mathrm{I}$ and ${ }^{131}$ I thyroid calibrations is shown in Figure 2.14. The head and neck phantom, manufactured by Humanoid Systems, Inc., ${ }^{2}$ is composed of a tissuesubstitute Adiprene material, incorporating a human skull and cervical vertebrae. The thyroid is a clear, molded acrylic, hollow unit, which can be filled with iodine solutions or a solid matrix and inserted into the cavity in the neck. This thyroid phantom is more tissue-equivalent than the acrylic neck phantom that

\footnotetext{
${ }^{2}$ Radiology Support Devices, formerly Humanoid Systems, Inc., 1904 East Dominguez Street, Long Beach, CA, 90810.
} 
is based on ANSI N44.3. However, based on Department of Energy Laboratory Accreditation Program (DOELAP) performance testing results for the IVMP counting procedure, the efficiency calibration based on measurements of a ${ }^{133} \mathrm{Ba}$ thyroid insert in the head and neck phantom can be used to estimate both ${ }^{125} \mathrm{I}$ and ${ }^{131}$ I activities in an ANSI N44.3 style phantom within $10 \%$.

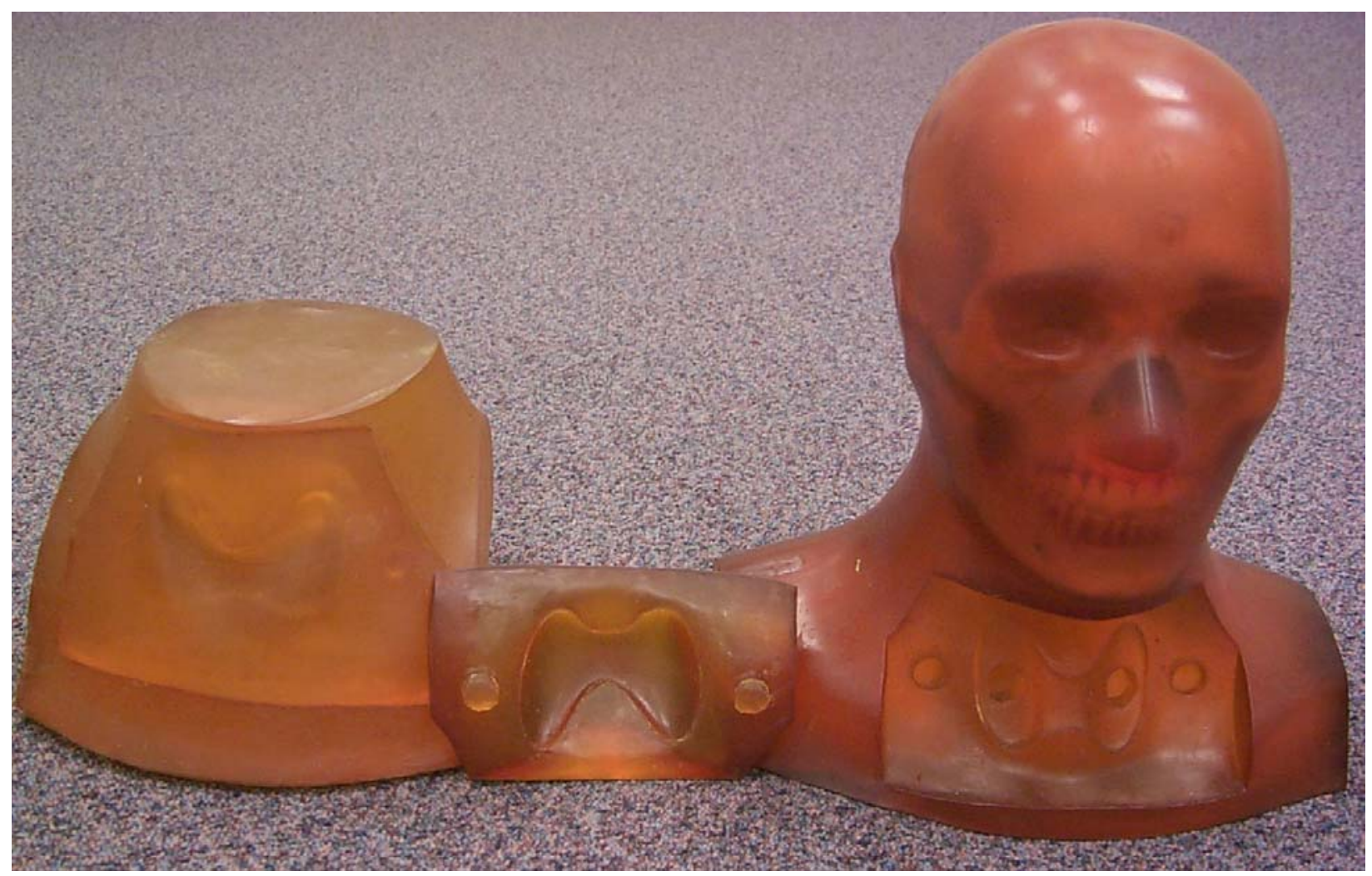

Figure 2.14. Humanoid Systems Thyroid Phantom

The neck phantom shown in Figure 2.14 can also be used for thyroid calibration measurements.

\subsection{Cited References}

American National Standards Institute (ANSI/HPS). 2009. Specifications for the Bottle Manikin Absorption Phantom. ANSI Standard N13.35. New York.

American National Standards Institute (ANSI). 1973. Thyroid Radioiodine Uptake Measurements Using a Neck Phantom. ANSI Standard N44.3. New York.

American National Standards Institute (ANSI/IEEE). 1986. IEEE Standard Test Procedures for Germanium Gamma-Ray Detectors. ANSI Standard 325. New York.

Eichner, F. N. 1969. Whole Body Counter Laboratory Mobile Unit B Description and Operation. BNWL-1154, Pacific Northwest Laboratory, Richland, Washington. 
Griffith, R. V., P. N. Dean, A. L. Anderson, and J. C. Fisher. 1978. "Fabrication of a Tissue-Equivalent Torso Phantom for Intercalibration of In-Vivo Transuranic-Nuclide Counting Facilities.” Paper presented at the Symposium on Advances in Radiation Protection Monitoring, June 26-30, 1978, Stockholm, Sweden.

Gunston, K. J., and S. J. Jeffries. 1986. Calibration of X-Ray Counters for Assessment of Internal Lung Con-tamination, with Low Energy X-Ray Emitters, in Women, Part I Measurements Made at AWRE.

Final Report to the International Atomic Energy Agency, Technical Contract 3362/TC., Vienna, Austria.

Hickman, D. P. and N. Cohen. 1988. "Reconstruction of a Human Skull Calibration Phantom Using Bone Sections from an ${ }^{241}$ Am Exposure Case,” Health Physics Journal, Vol. 55, No. 1.

ICRP Publication 23. Report of the Task Group on Reference Man. 1975. International Commission on Radiological Protection. Pergamon Press. London.

Kephart, G. S. 1987. “An Arm Phantom for In Vivo Determination of Americium-241 In Bone,” Master’s Thesis, University of Washington.

Knoll, G. F. 2000. Radiation Detection and Measurement. 3rd ed. John Wiley and Sons, New York.

McInroy, J. F., H. A. Boyd, B. C. Eutsler, D. Romero. October 1985. “The U.S. Transuranium Registry Report on the ${ }^{241}$ Am Content of a Whole Body,” Health Physics Journal, Vol. 49, No.4, Part IV.

Newton, D., A. C. Wells, S. Mizushita, R. E. Toohey, J. Y. Sha, R. Jones, S. J. Jeffries, H. E. Palmer, G. A. Rieksts, A. L. Anderson, and G. W. Campbell. 1985. "The Livermore Phantom as a Calibration Standard in Assessment of Plutonium in Lungs." In Proceedings of an International Symposium on Assessment of Radioactive Contamination in Man, pp. 183-199. IAEA-SM-276/01, International Atomic Energy Agency, Vienna.

Roesch, W. C., R. C. McCall, and H. E. Palmer. 1960. Hanford Whole Body Counter 1959 Activities. HW-67045, Hanford Atomic Products Operation, General Electric Company, Richland, Washington.

Snyder, S.F., Traub, R.J. March 2010. “The Livermore Phantom History and Supplementation”, Health Physics Journal, Vol. 98, No.3.

Swanberg, Jr., F. 1963. Hanford Mobile Whole Body Counter. HW-80216, Hanford Atomic Products Operation, General Electric Company, Richland, Washington.

Taylor, F.V. 1997 History of the Lawrence Livermore National Laboratory Torso Phantom. Master's thesis. UMI Dissertation Publishing, Ann Arbor, Michigan 48106-1346.

\subsection{Other Useful References}

Palmer, H. E. and W. C. Roesch. 1965. "A Shadow-Shield Whole Body Counter.” Health Physics 11(11):1213-1219. 
IN VIVO MONITORING PROGRAM MANUAL

$$
\text { PNL-MA-574 }
$$

SECTION 3.0

IN VIVO MEASUREMENT SYSTEMS 


\section{Contents}

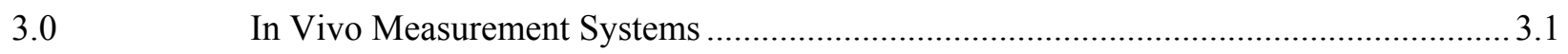

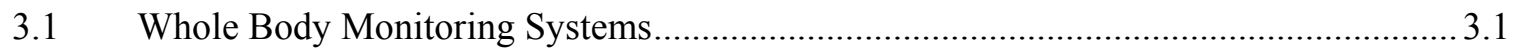

3.1.1 Standup Whole Body Counters................................................................. 3.1

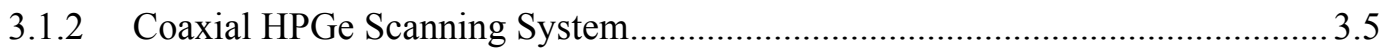

3.1.3 Shadow Shield Counting Systems ................................................................ 3.5

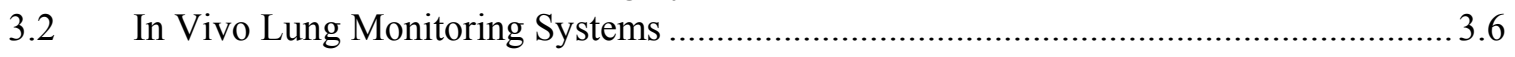

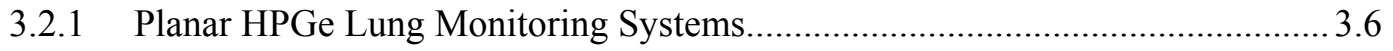

3.2.2 High-Energy Photon Lung-Monitoring Systems …........................................... 3.10

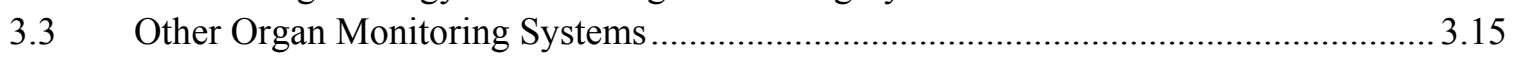

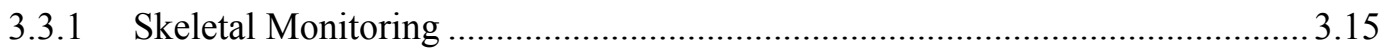

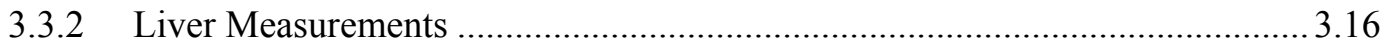

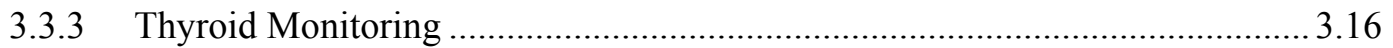

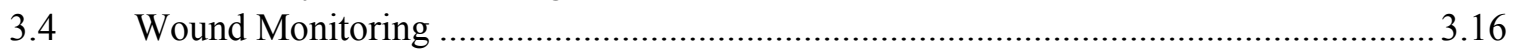

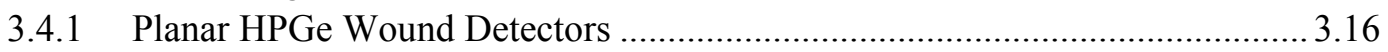

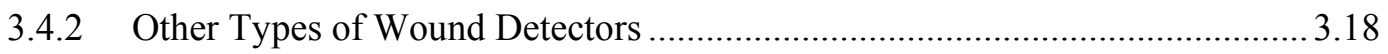

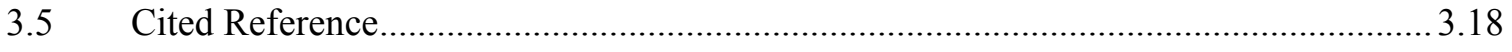

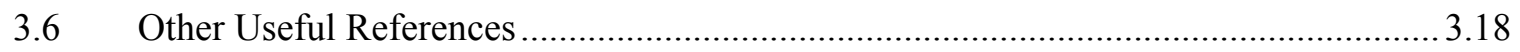




\subsection{In Vivo Measurement Systems}

Different types of measurement systems are used for the identification and quantification of radioactive material in the human body depending on the radionuclide. Measurements are made to estimate the radionuclide content of specific organs, such as the lung, liver, or skeleton, or the entire body of the subject. This chapter contains a discussion of the various measurement systems and their functions, components, and operating characteristics.

\subsection{Whole Body Monitoring Systems}

Whole body measurements are the most frequently performed measurements at the In Vivo Radiobioassay and Research Facility (IVRRF). NaI and high purity germanium (HPGe) detection systems are used to quantify the activity of radionuclides that emit high-energy electromagnetic radiations. Due to the age of the waste at Hanford the fission product activity predominates compared to activation product activity. Cesium-137 is used as an indicator for potential plutonium intakes for certain work locations where the fission product activity predominates but the transuranic activity has the greatest dose consequences. The measurement protocols using the coaxial HPGe system and the standup counter in the 747A Building are accredited through the Department of Energy Laboratory Accreditation Program (DOELAP).

\subsubsection{Standup Whole Body Counters}

Two counting systems are designed to provide rapid and sensitive measurements of high-energy gamma rays emitted from the body. These standup whole body counters are so named because of the orientation a worker assumes to perform the measurement. One counter is located in the 747A Building, and the other is in a mobile trailer. These counters are used for screening measurements to determine whether activity is present above the decision level value.

The standup counter in the 747A building consists of five $\mathrm{NaI}(\mathrm{Tl})$ scintillation detectors in a vertical array. The orientation of the detectors is "end-on" with the flat face of the detector crystals facing the subject. Figure 3.1 shows the detector orientation and the plexiglas barrier that supports the subject and that protects the detector face from damage. The five $\mathrm{NaI}(\mathrm{Tl})$ detectors include four with $23.8-\mathrm{cm}(9.375-\mathrm{in}$.) diameter crystals and one with a $29.2-\mathrm{cm}$ (11.5-in.) diameter crystal. The larger crystal is positioned behind the chest and the four smaller crystals are arranged behind the head, abdomen, thighs, and calves by means of the counter-weighted mounting frame.

The standup counter in the 747A building has separate high-voltage supplies, preamplifiers, and main amplifiers for each detector. The amplifier outputs are directed to specific channels in a mixer/router, then to the analog to digital converter (ADC), and finally to the multichannel analyzer (MCA) memory. A workstation displays the five separate spectra during acquisition. A block diagram of the system is shown in Figure 3.2. A plot of a typical counting efficiency curve for the counter is displayed in Figure 3.3. 


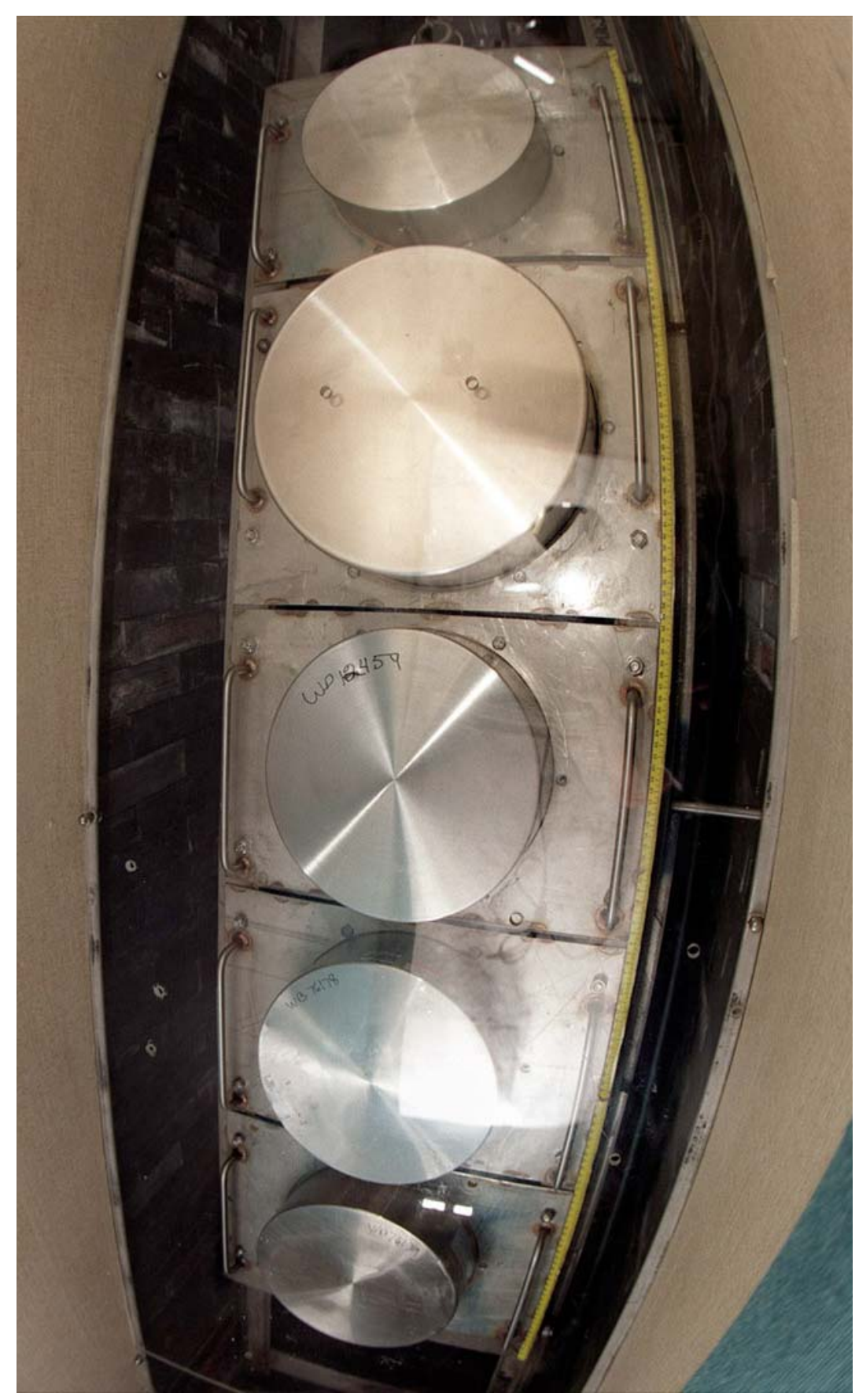

Figure 3.1. Standup Whole Body Counter at IVRRF 


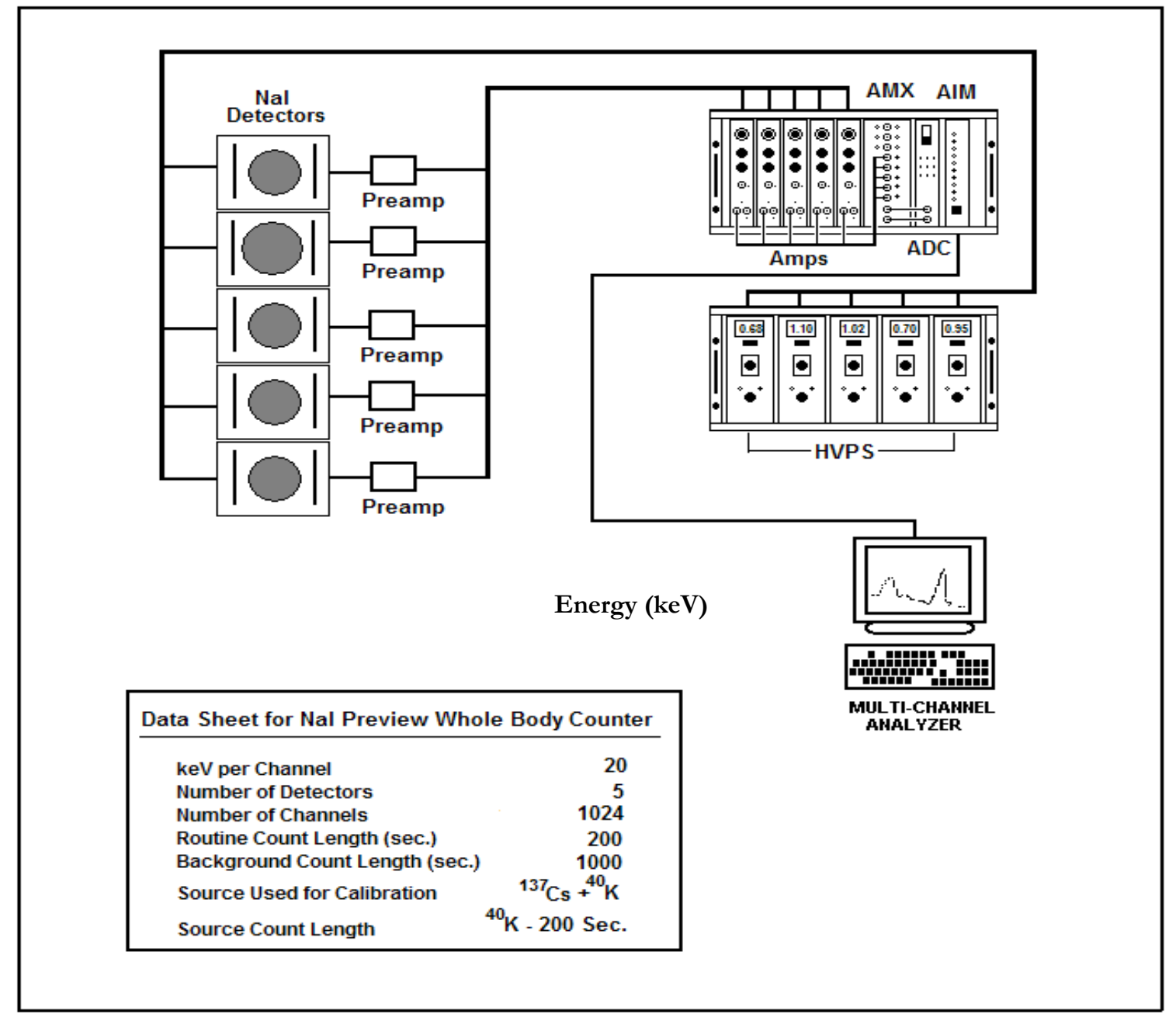

Figure 3.2. Block Diagram of the Standup Counter Configuration

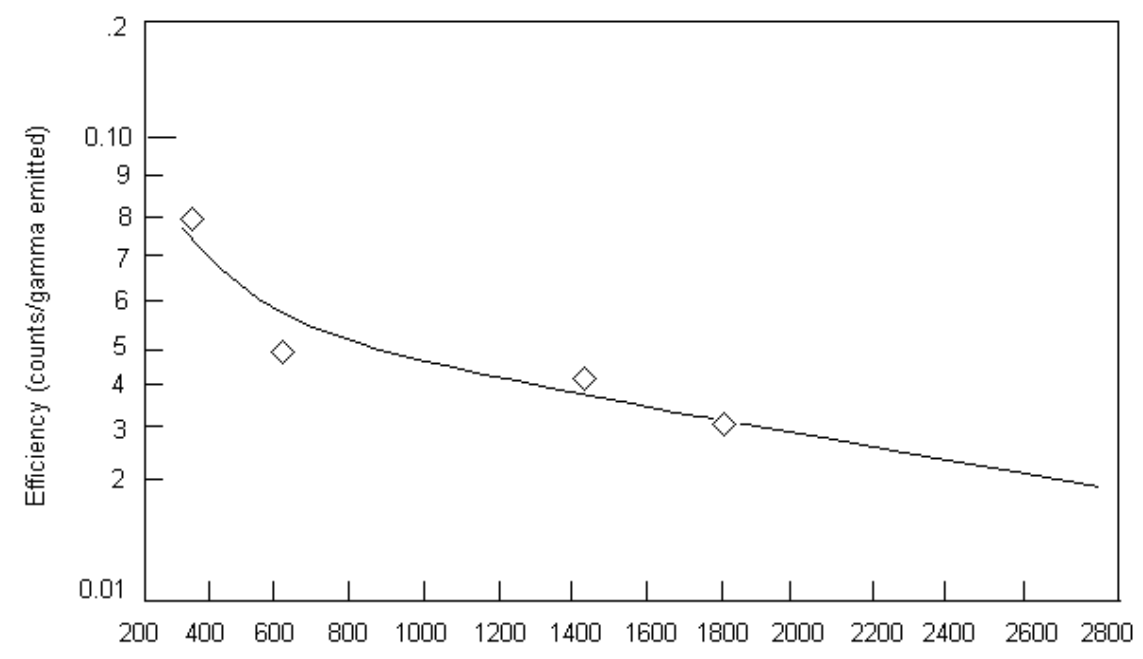

Figure 3.3. Example Standup Whole Body Counter Efficiency Calibration Curve 
A second standup counter using a different source-to-detector geometry is located in the mobile trailer as shown in Figure 3.4. This system is currently maintained in a standby mode. Its construction is similar to the standup counter in the 747A Building, but was designed for minimum weight so that it could be transported without exceeding transportation load limits. The counter is designed for six rectangular $\mathrm{NaI}(\mathrm{Tl})$ detectors, five with dimensions of $10 \times 10 \times 40 \mathrm{~cm}(4 \times 4 \times 16$ in.) and the sixth is $10 \times 10 \times 20 \mathrm{~cm}(4 \times 4 \times 8$ in. $)$. The fixed array of detectors is $168 \mathrm{~cm}$ (66 in.) high; the top of the array is $183 \mathrm{~cm}(72 \mathrm{in}$.$) above the floor. A 1-\mathrm{cm}$ (0.375-in.) sheet of acrylic plastic separates the detectors and the subject being counted. The total area of the $10-\mathrm{cm}(3.75$-in.) thick detectors is $2271 \mathrm{~cm} 2$ (352 in.2), compared to the 747A standup counter area of $2387 \mathrm{~cm} 2$ (370 in.2). The efficiency of the system is $15 \%$ lower for $40 \mathrm{~K}$ compared to the $747 \mathrm{~A}$ counter because of the smaller area of the detectors, a reduced number of photomultiplier tubes (PMTs), and a different shielding arrangement. The background contribution in the $40 \mathrm{~K}$ peak regions of interest (ROI) is $33 \%$ lower, primarily due to the smaller number of PMTs.

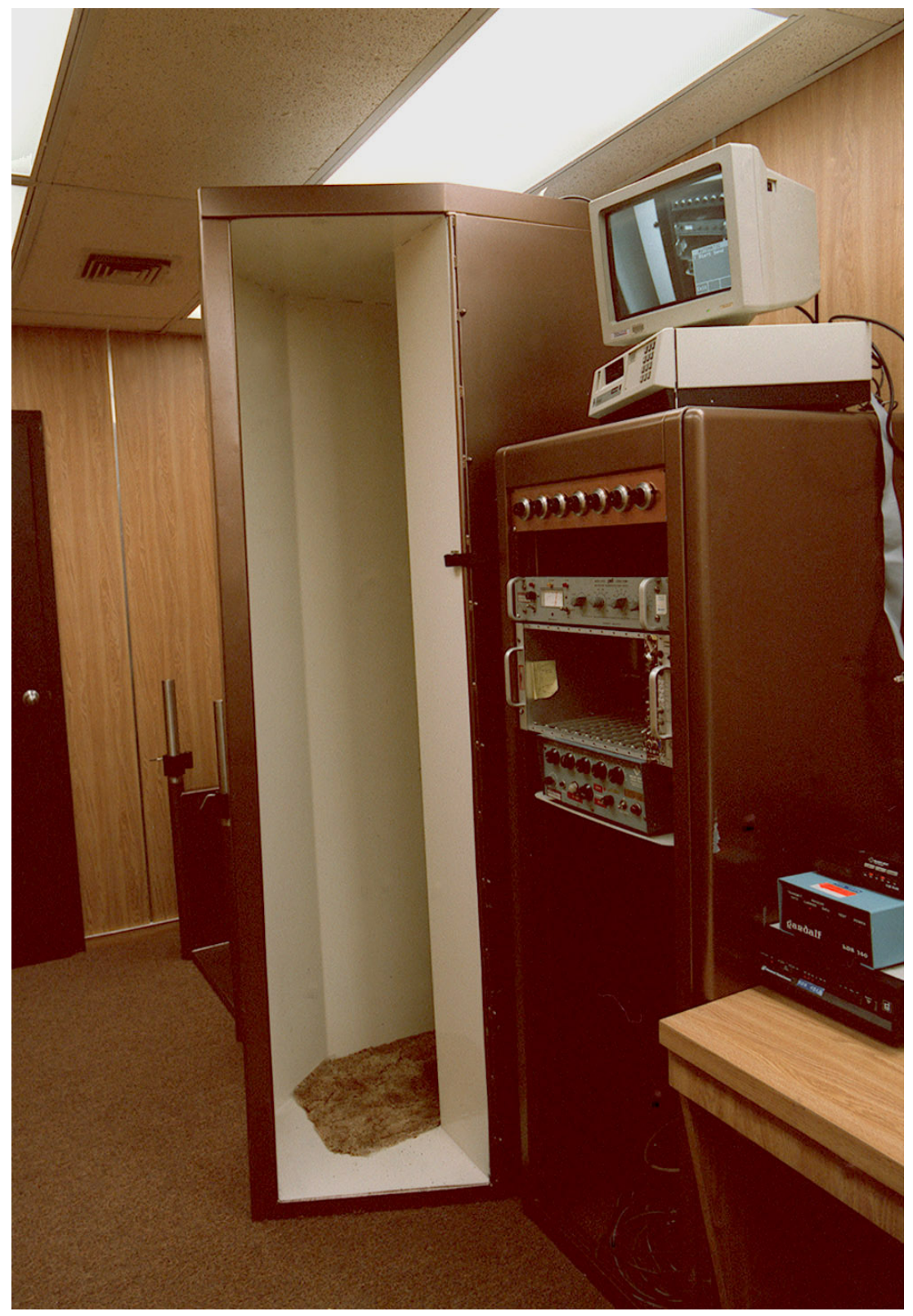

Figure 3.4. Mobile Standup Whole Body Counter 


\subsubsection{Coaxial HPGe Scanning System}

The coaxial HPGe scanning system is located in the Palmer Room. It consists of an array of five p-type coaxial HPGe detectors with efficiencies ranging from $120 \%$ to $130 \%$. Two of the detectors share a common 50 liter dewar with the crystals incorporated a vertical cryostat configurations. The other three detectors also share a common 50-liter capacity dewar and are configured within horizontal cryostats that have their long axis parallel to the long axis of the worker's body in the prone position. Figure 3.5 illustrates the configuration of the HPGe

array. The dewars, cryostats and associated electronics are mounted on a steel frame that is positioned underneath the counting platform. The frame is coupled to a servomotor powered drive system that is computer controlled. Scan times can be set from 60 seconds to 8 hours. The servomotor controller can store up to 7 counting sequences in a non-volatile memory. The detectors travel under the subject during the counting procedure. There is also the capability to perform measurements with the detectors stationary.

Each of the output signals from the detectors is routed to a separate digital spectrum analyzer (DSA). The DSA is an integrated multichannel analyzer that includes the high voltage power supply and network interface. The DSAs are controlled from the main computer console using the Abacos Plus software from Canberra Industries. The block diagram and counting parameters for the system are shown in Figure 3.6. A plot of typical counting efficiency curves is shown in Figure 3.7.

\subsubsection{Shadow Shield Counting Systems}

A horizontal shadow shield counter, a design developed in the early 1960s by H. Earl Palmer and colleagues, is available as a back-up to the routine measurement systems. This whole-body counter is designed (Palmer and Roesch 1965) to provide sensitive and accurate measurements of radionuclides in the body that emit photons with energies greater than $200 \mathrm{keV}$. The annular lead rings surround all but one side of the NaI detector, effectively collimating the field of view of the detector. Shielding is also provided under and on the sides of the counting platform. Consequently, only photons originating from the subject can enter the detector without passing through the shield or undergoing at least one scattering event. The shield is composed of lead bricks enclosed in a sheet metal cover and weighs approximately $4550 \mathrm{~kg}$ (5 tons). The detector used in these counters is a cylindrical 23 $\mathrm{cm}$ by $10 \mathrm{~cm} \mathrm{NaI}(\mathrm{Tl})$ scintillation crystal. The subject is moved beneath the detector on a motor driven sled. The shadow shield counter is located in the mobile trailer and is currently in a standby mode. Figure 3.8 shows the shadow shield counter. The integral scan that is performed on the subject has a 10-min count length, and the detector pulses are routed to a portable multichannel analyzer (MCA) after being properly amplified and converted. Figure 3.9 displays the block diagram of this shadow shield counter. 


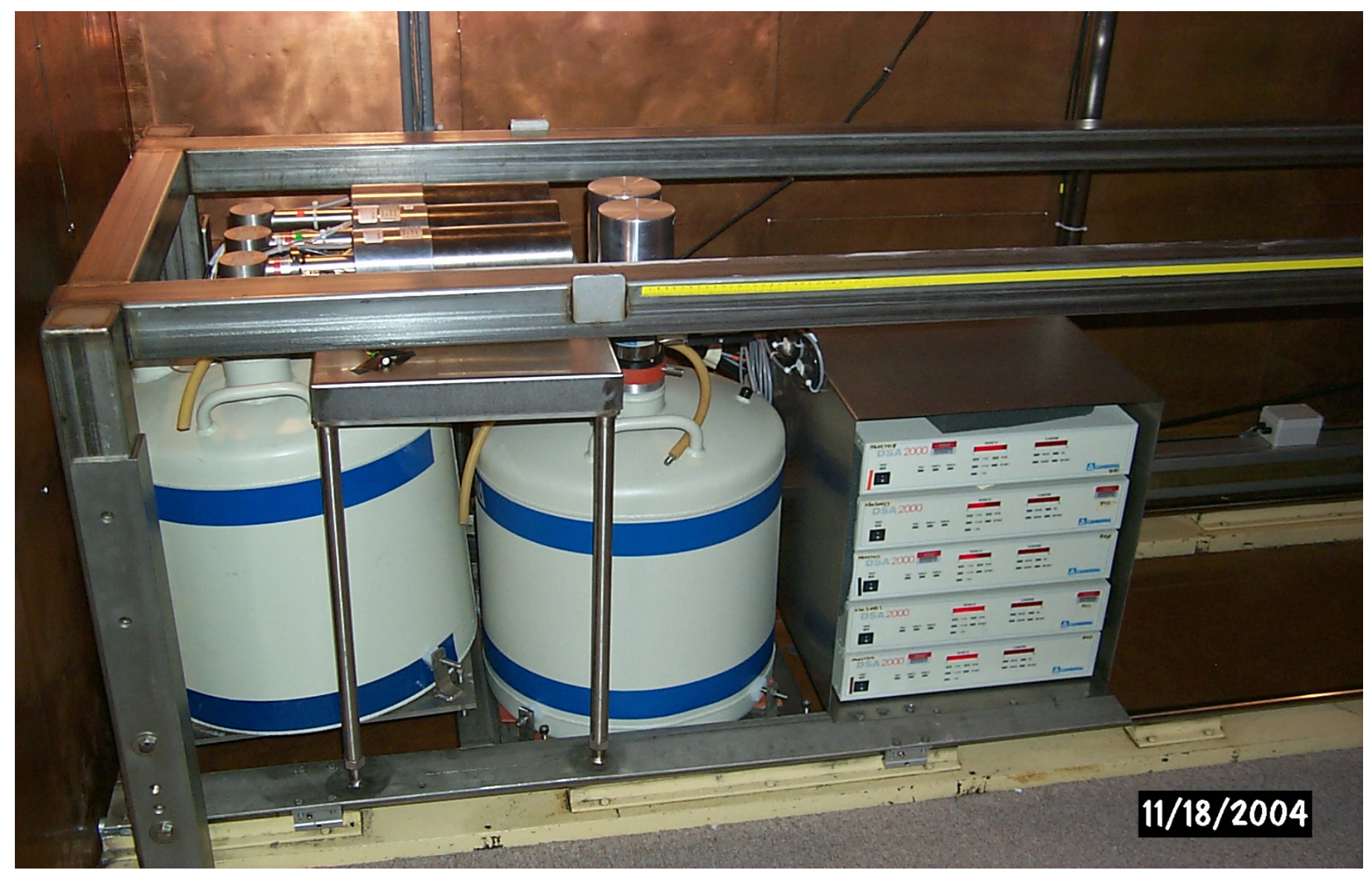

Figure 3.5. Coaxial HPGe Scanning Array

\subsection{In Vivo Lung Monitoring Systems}

There are three in vivo measurement systems used for lung monitoring of radionuclides that emit low-energy photons. Each of the systems are DOELAP accredited for measurements of Department of Energy (DOE) and DOE contractor workers. The three systems are also used for measurements of non-DOE workers.

The coaxial HPGe and stand-up counters are used for the measurement of high-energy photons from radioactive material deposited in the lungs. The coaxial HPGe system is DOELAP accredited for measurement of fission and activation products in the lungs.

\subsubsection{Planar HPGe Lung Monitoring Systems}

Two of the low-energy lung-monitoring systems each include four planar HPGe detectors and associated electronics. They are used primarily to detect radioactive material that is deposited in the respiratory tract of workers. They are designed and operated to detect photons with energies $<500 \mathrm{keV}$. For routine operations the $59.5 \mathrm{keV}, 63.3 \mathrm{keV}$, and the $185.7 \mathrm{keV}$ photons are used for calculating the $241 \mathrm{Am}, 234 \mathrm{Th}$, and $235 \mathrm{U}$ activities respectively. The subject is seated in a semi-reclining position in an adjustable chair and measurements are made with the subject's chest in light contact with the detectors' entrance windows. 
The two lung monitoring systems have similar cryostat configurations and have similar signal processing electronics. The systems are located in the Stainless Steel and Iron Rooms.

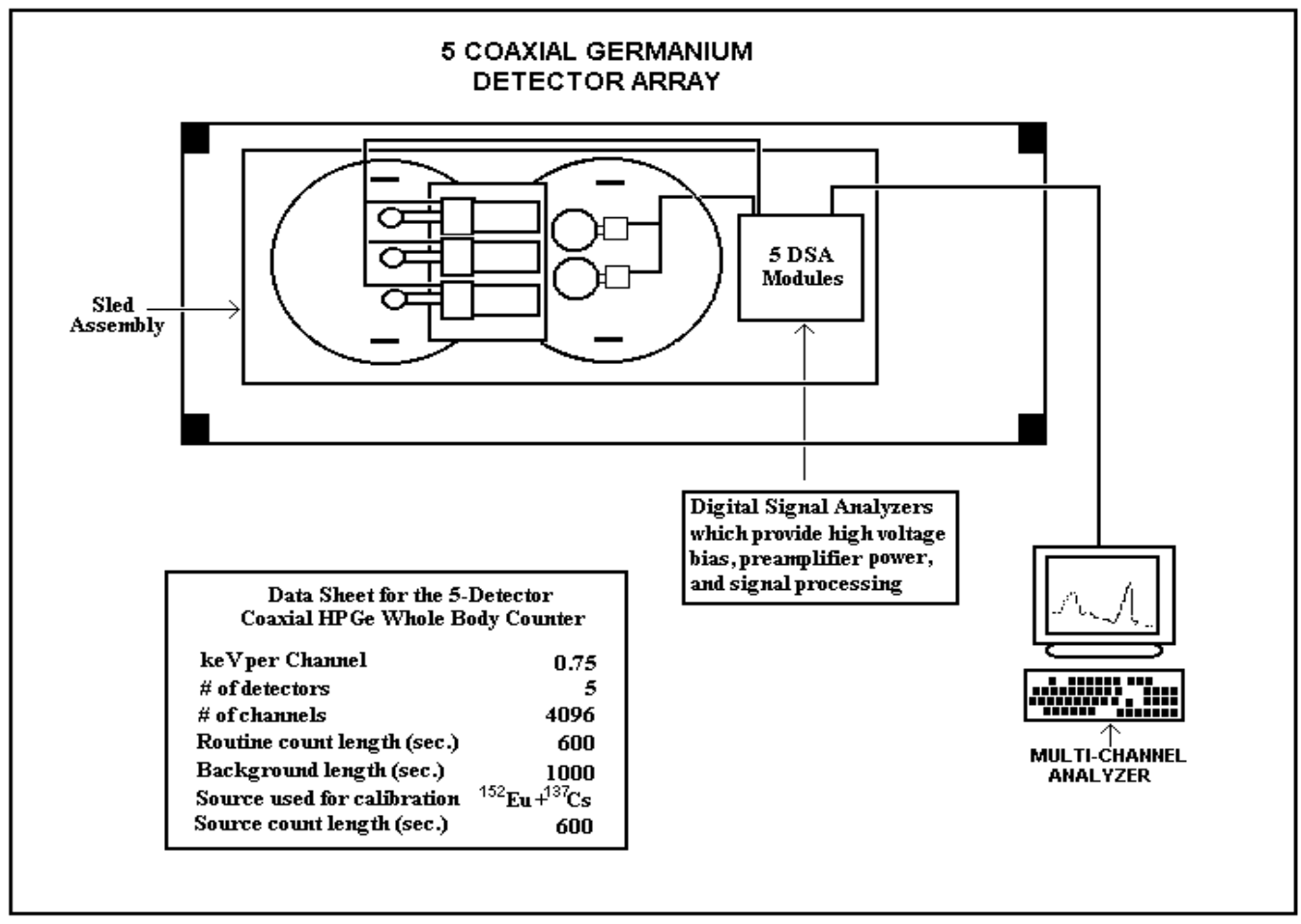

Figure 3.6. Block Diagram and Typical Counting Parameters for Coaxial HPGe Scanning System

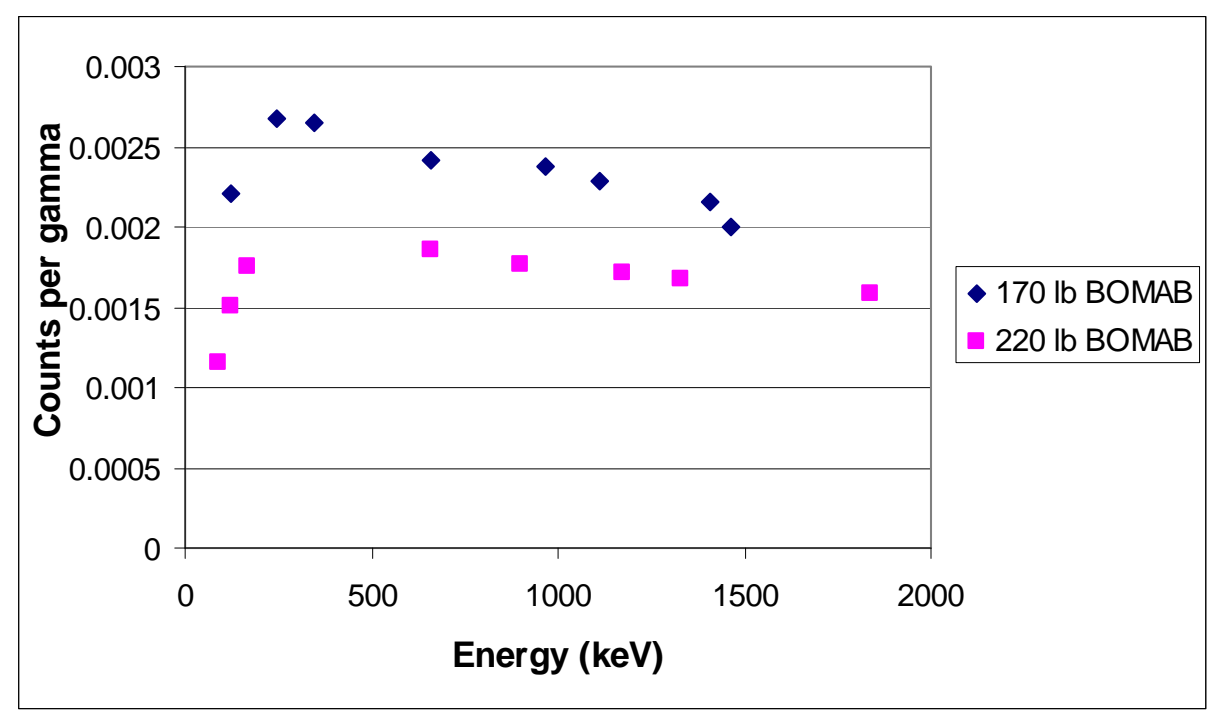

Figure 3.7. Efficiency Curves for the Coaxial HPGe Scanning System 


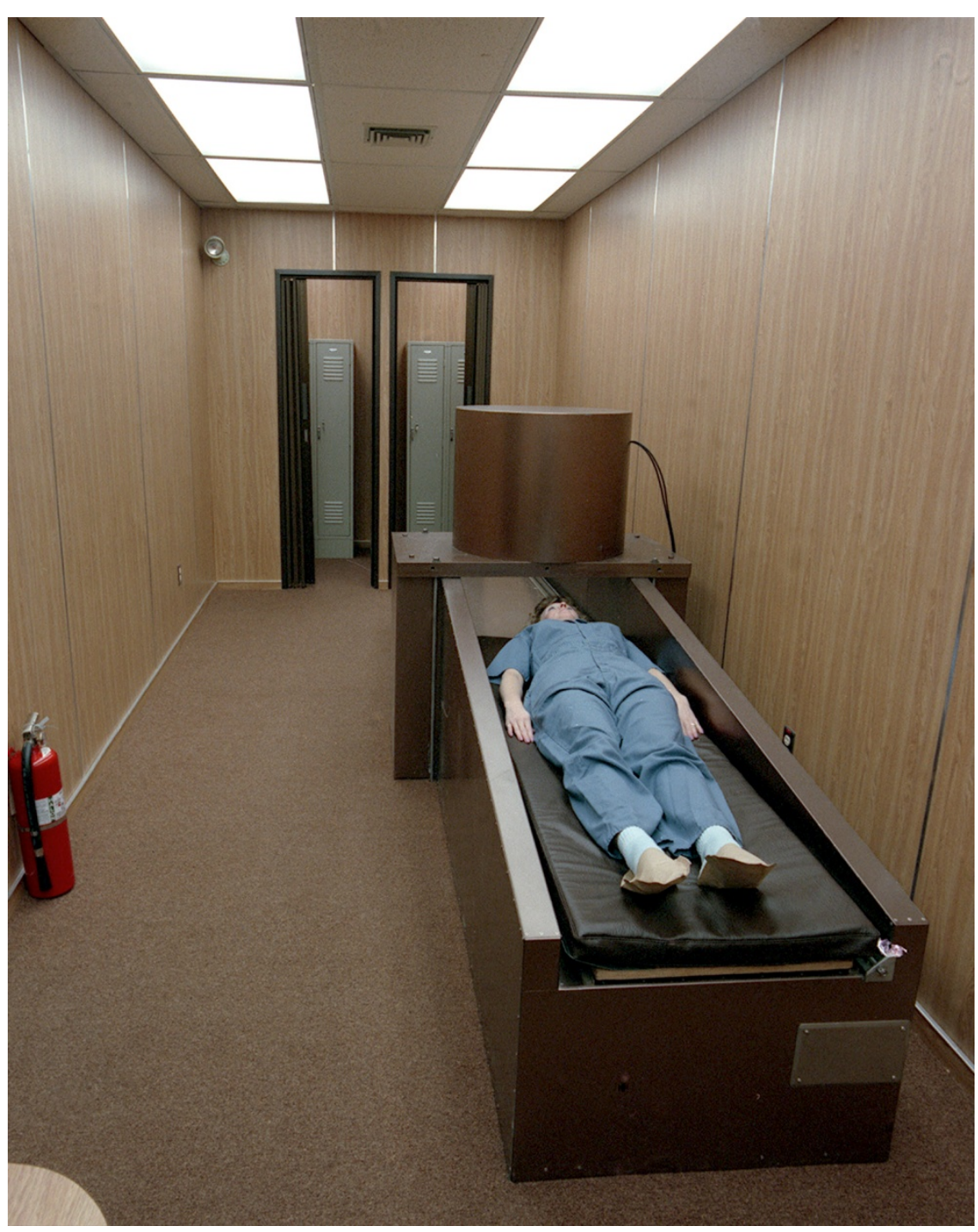

Figure 3.8. Mobile Shadow Shield Whole Body Counter

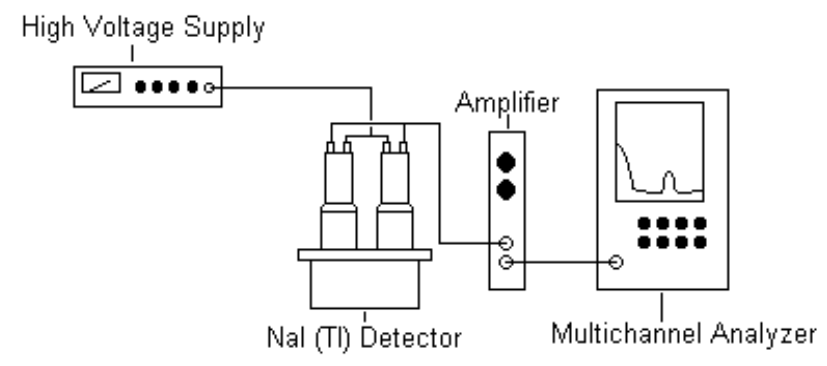

Figure 3.9. Block Diagram and Counting Parameters for a Shadow Shield Counter 


\subsubsection{Stainless Steel Room}

The lung monitoring system in the Stainless Steel Room incorporates four 38-cm2 N-type planar HPGe detectors. Two detectors have $20 \mathrm{~mm}$ thick crystals and two detectors have a crystal thickness of $30 \mathrm{~mm}$. Each detector has a $0.75 \mathrm{~mm}$ thick carbon fiber entrance window. The four detectors are oriented with the entrance windows in a common plane for the worker measurements and calibration measurements. There is a 1 to 1.5 inch latitudinal separation between the two left detectors and the two right detectors.

The detector-mounting system was fabricated at the Pacific Northwest National Laboratory (PNNL) to support the four 20-L dewars that contain liquid nitrogen used to maintain the cryogenic operating temperature required by the detectors. The mounting system allows limited adjustments to position the individual detectors. Adjustments can also be made to the attitude of the entire four-detector array. The chair in the counting room has adjustments for additional height and attitude adjustments to optimize the counting geometry for the worker.

Figure 3.10 illustrates the detection system in the Stainless Steel Room. Figure 3.11 contains a block diagram of the system including a list of the typical counting parameters.

Each of the four detectors is interfaced with a DSA that supplies the high-voltage and signal processing electronics. The bias potential for the detectors is typically $-2000 \mathrm{~V}$ to $-3000 \mathrm{~V}$. The preamplifier output is promptly digitized, amplified, filtered, shaped and stored in the appropriate DSA memory location. The spectra are displayed from a computer console. The individual detector spectra are acquired and processed separately; the four spectra are then electronically summed to form the composite spectrum that is used for the activity calculations.

\subsubsection{Iron Room}

The Iron Room lung monitoring system is quite similar in design and equipment to the Stainless Steel Room. The system also includes four N-type planar HPGe detectors with similar characteristics as the Stainless Steel Room. The primary differences are that all four detector crystals are $30 \mathrm{~mm}$ thick and that the detectors in the Iron Room have beryllium entrance windows. The detectors with the beryllium windows have a somewhat higher uranium background compared to the detectors with carbon composite windows. 


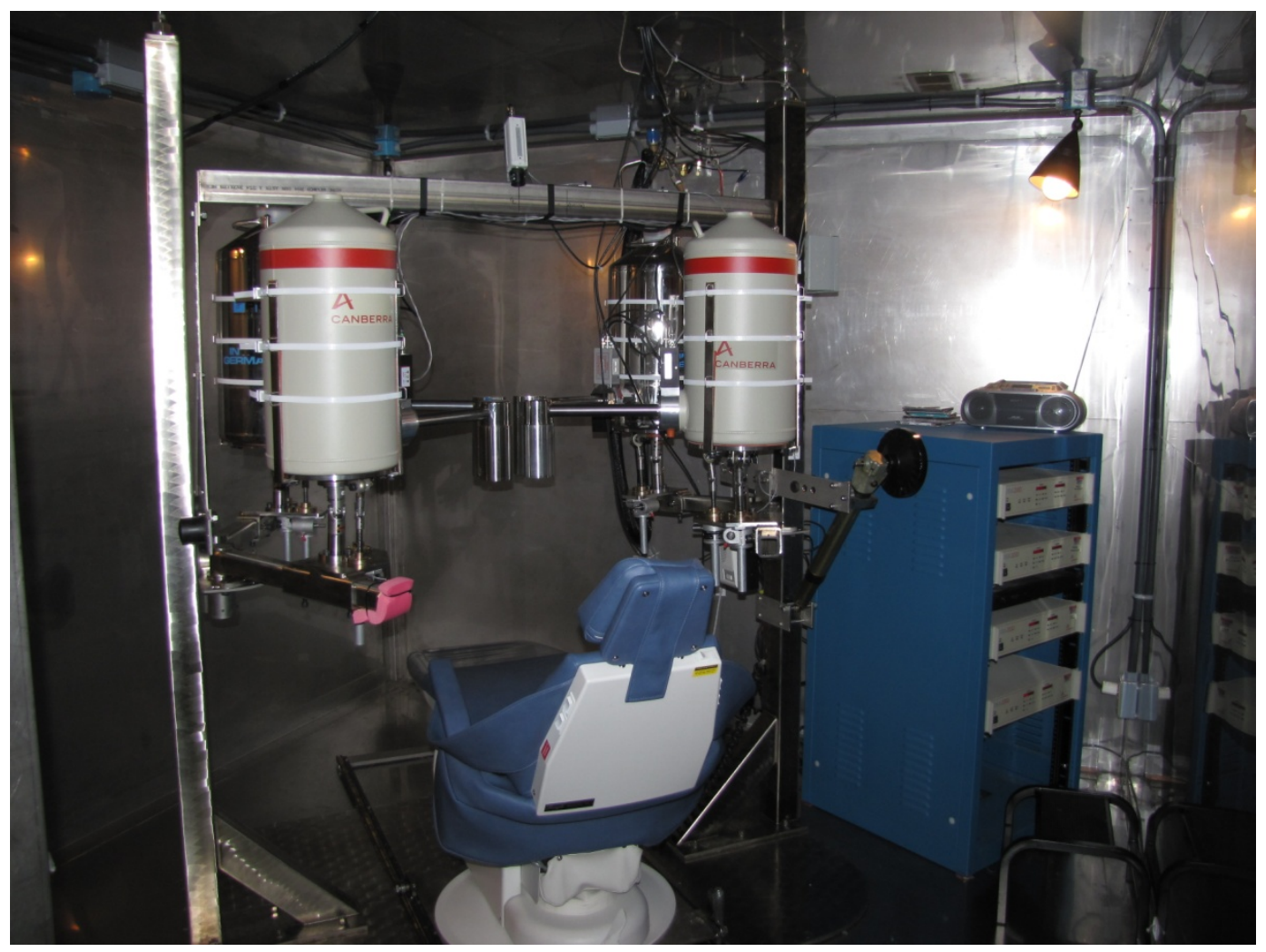

Figure 3.10. Stainless Steel Room In Vivo Lung Monitoring System

Figure 3.12 depicts the detection system in the Iron Room. Figure 3.13 contains the block diagram and counting parameters for the system. The calibration curve for both systems is expressed as the efficiency at a specific energy as a function of the chest wall thickness. Typical calibration curves used to quantify 241Am and 235U activity in the lungs are shown in Figure 3.14.

\subsubsection{Lead Room}

The Lead Room houses the third system used for low energy lung measurements of Hanford workers. It is also used for measurements of non-DOE clients The system includes two N-type planar germanium detectors. A technical equivalence request was submitted to DOELAP in August 2009. This system was determined to be technically equivalent to the Iron Room and Stainless Steel Room systems and is included under the terms and conditions of the DOELAP accreditation as described in the November 9, 2009 letter from the DOELAP administrator. This system is also configured as needed for measurements of skeletal activity, wound activity and thyroid activity. The detector crystals are $7 \mathrm{~cm}$ in diameter with one crystal $2 \mathrm{~cm}$ thick and the other $3-\mathrm{cm}$ thick. Figure 3.15 depicts the Lead Room detection system.

\subsubsection{High-Energy Photon Lung-Monitoring Systems}

The coaxial HPGe scanning system and the standup whole body counter can be calibrated for measurements of radionuclides in the lungs that emit high energy photons. These types of lung measurements are made with the detectors positioned posterior to the worker and are calibrated with a torso phantom that contains simulated scapulae. The coaxial HPGe array in the Palmer Room can also be operated in a stationary mode for in vivo lung monitoring as described below. 


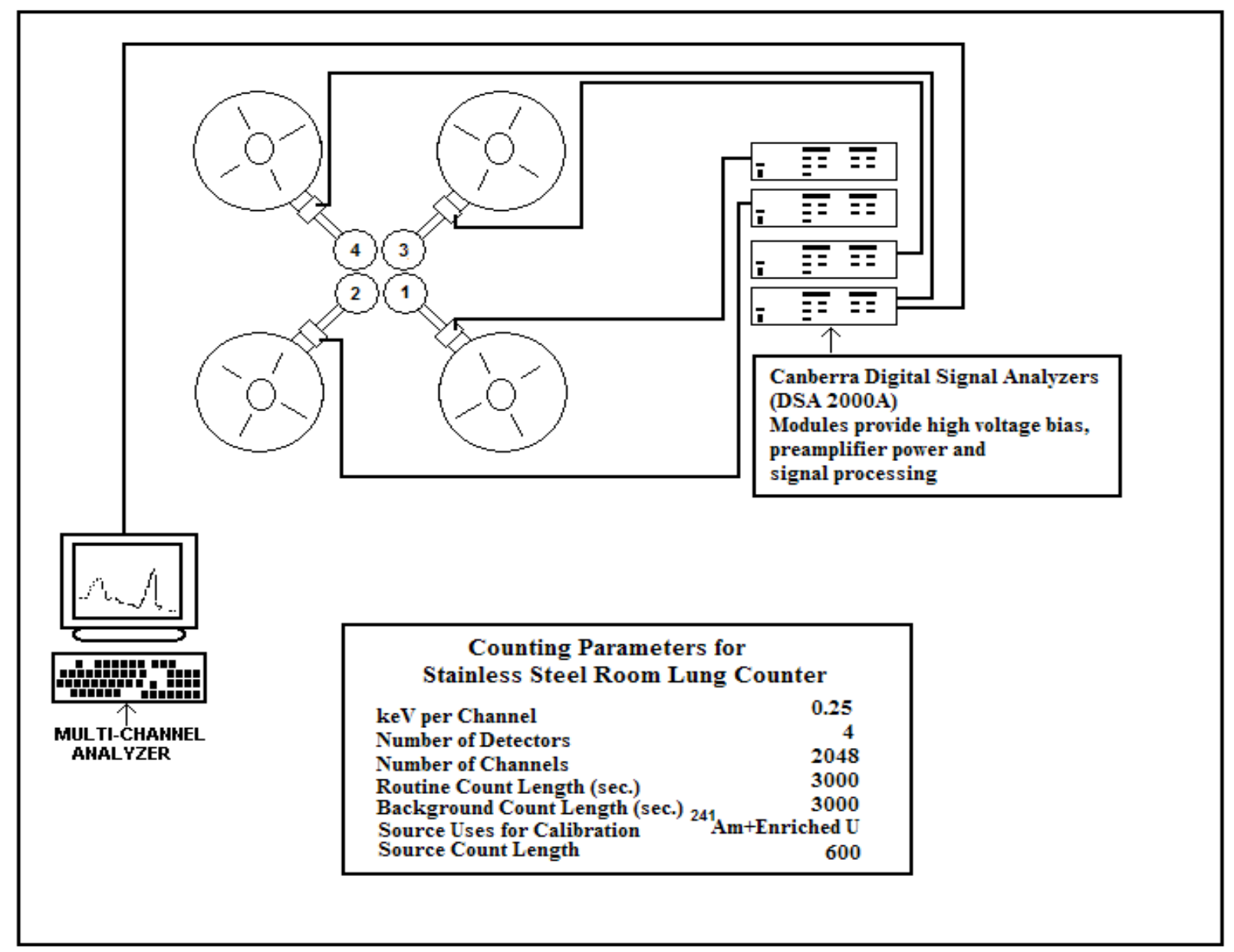

Figure 3.11. Block Diagram and Typical Counting Parameters of the Stainless Steel Room 


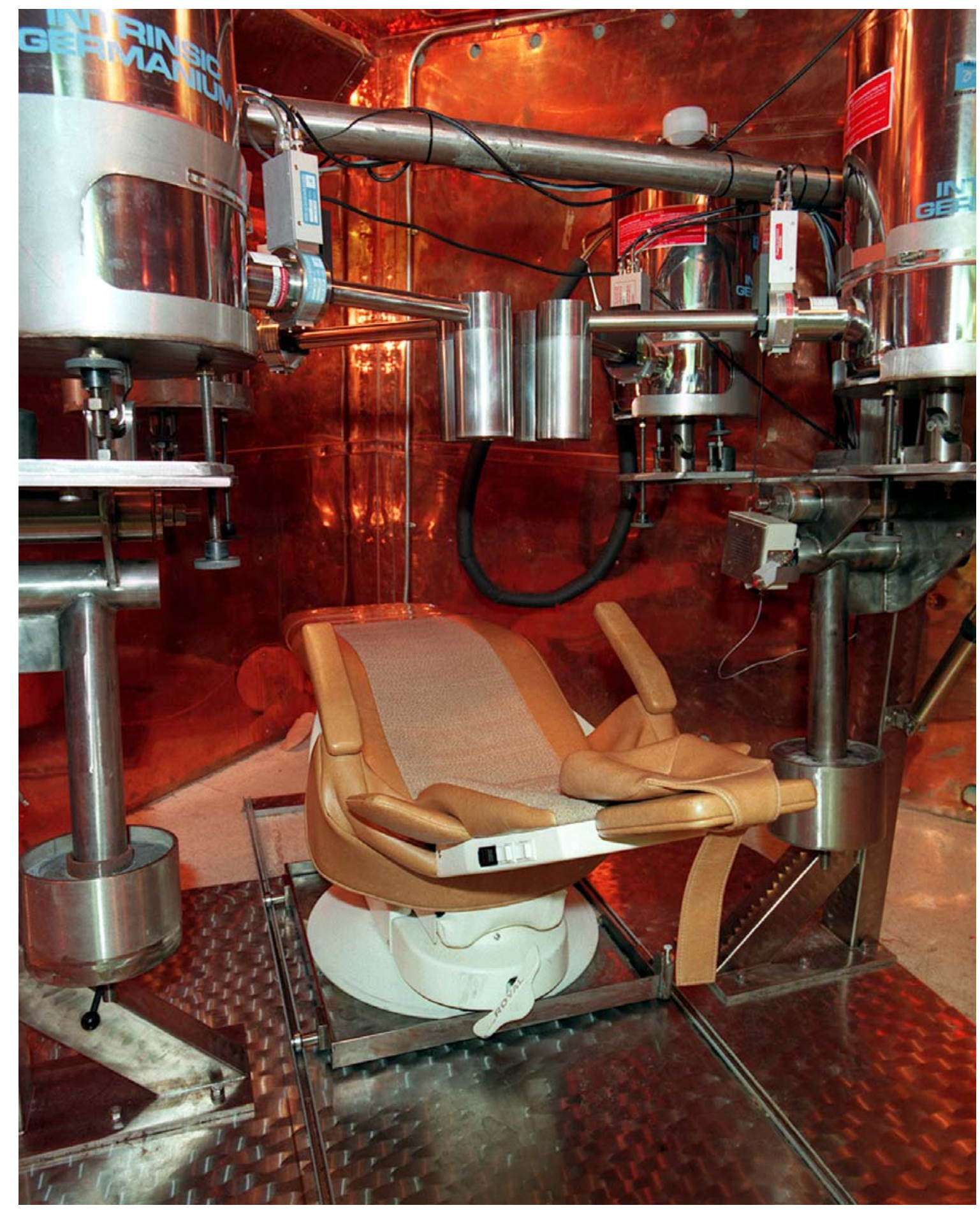

Figure 3.12. Iron Room In Vivo Lung Monitoring System 


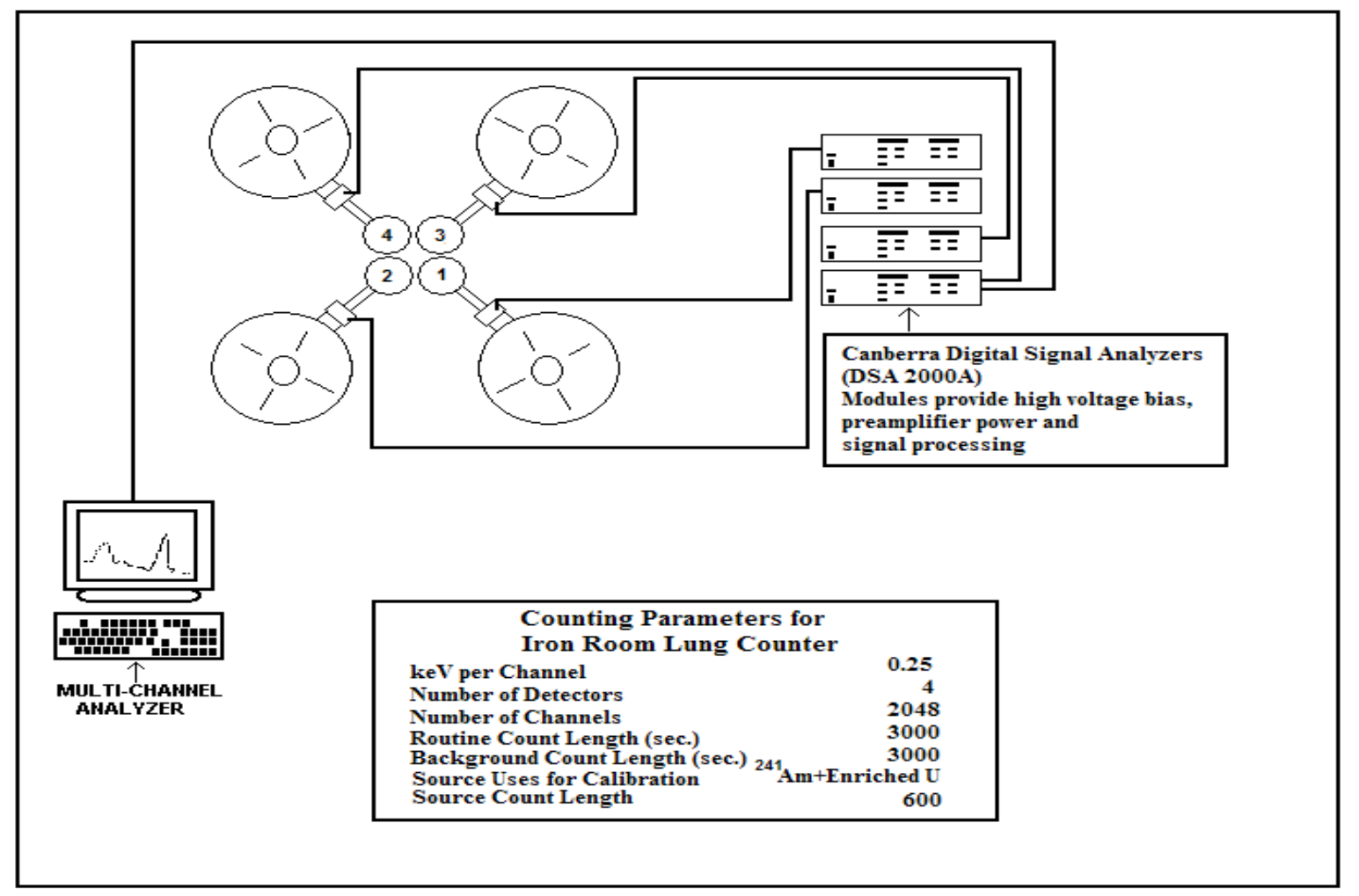

Figure 3.13. Block Diagram and Typical Counting Parameters for the Iron Room 


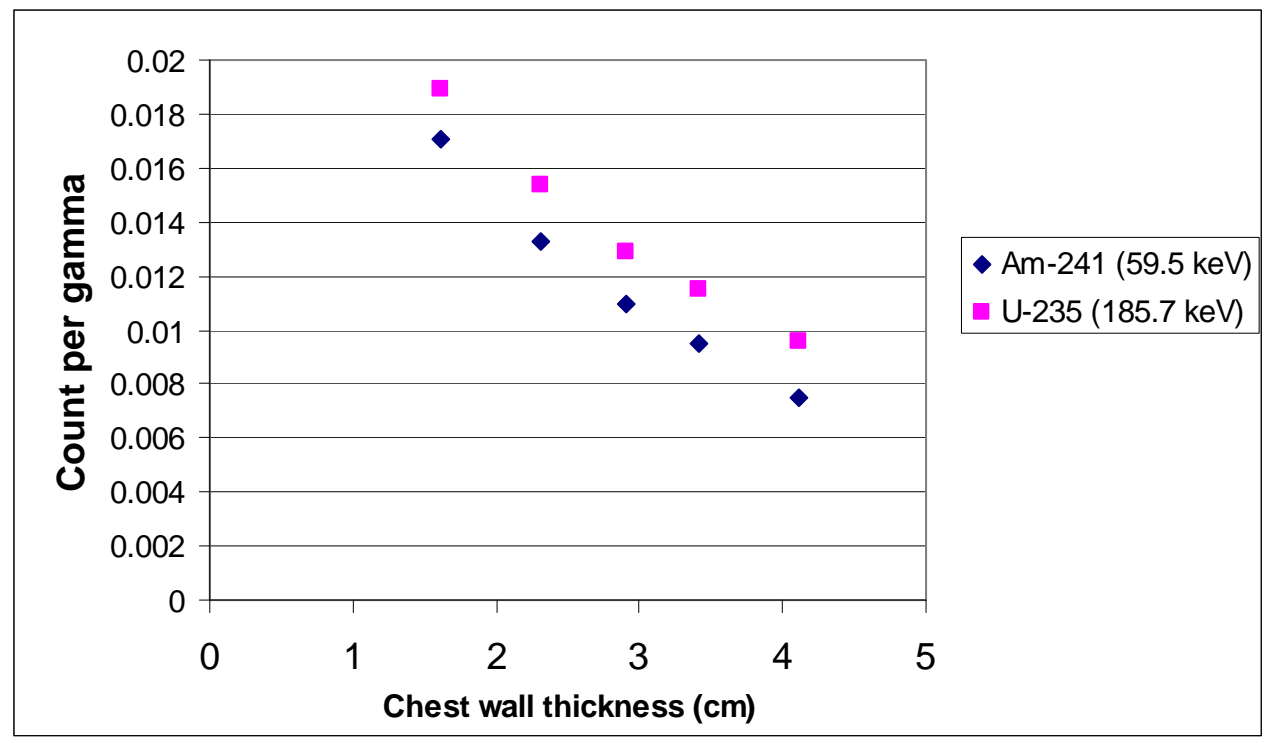

Figure 3.14. Example Efficiency Curves Used for Lung Measurements

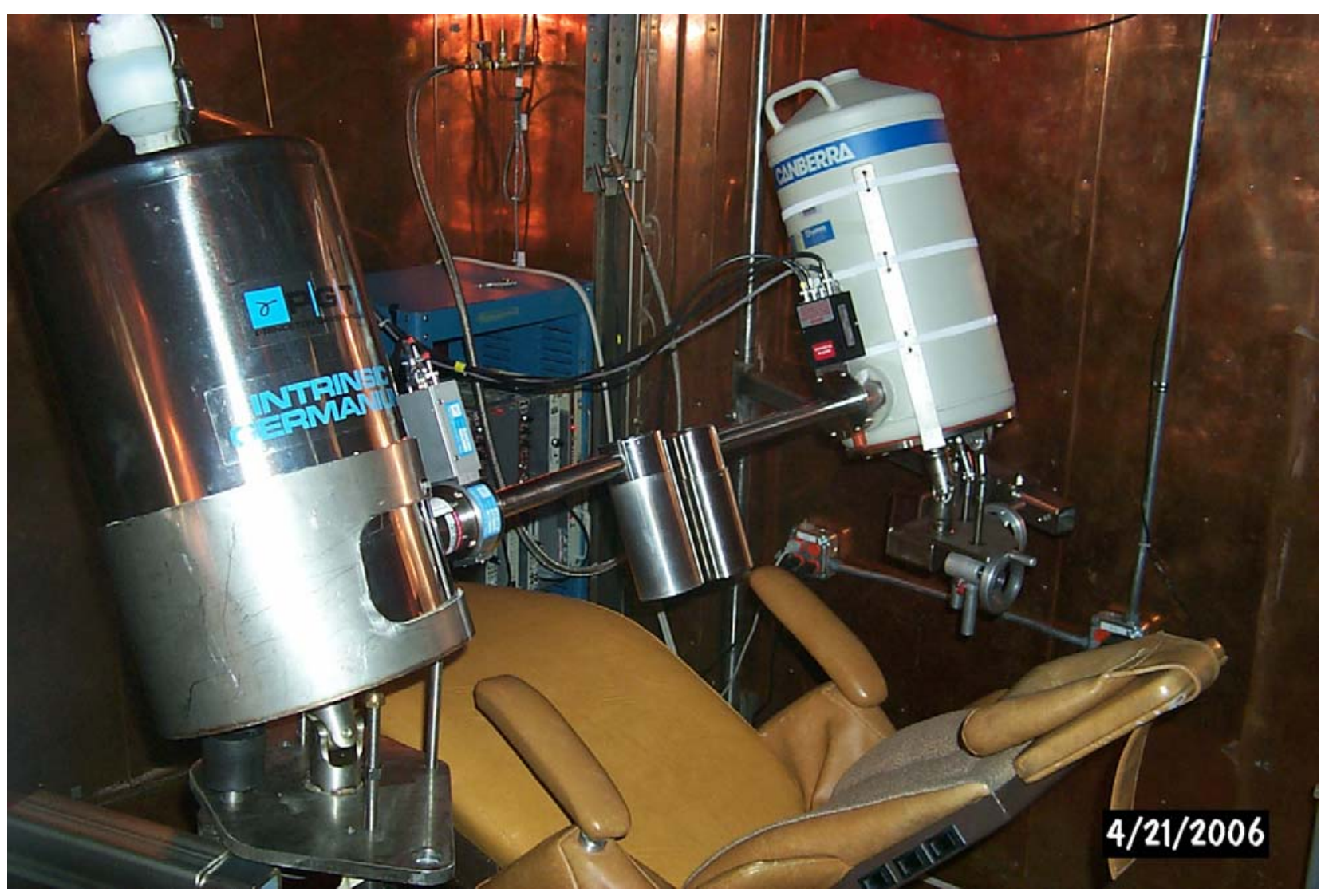

Figure 3.15. Lead Room Counting System 


\subsubsection{Coaxial HPGe Lung Measurements}

The coaxial HPGe system is usually used in a scanning mode for routine whole body measurements. The scanning measurement is also used to estimate the quantity of radioactive material in the lungs. It can also be operated in a stationary mode for in vivo measurement of radioactive material in the lungs to obtain a lower minimum detectable activity (MDA). To do this the detectors are centered under the subject's lungs and the motion-control system is deactivated. The measurement is performed using an appropriate counting time, with all the remaining count parameters being identical to the coaxial scanning measurement discussed in Section 3.1.2. The range of photon energies that can be analyzed with this system is from $100 \mathrm{keV}$ to $3 \mathrm{MeV}$.

\subsubsection{Standup Counter Lung Measurements}

The standup whole body counter can also be used to perform measurements of high-energy gamma rays emanating from the lung. A calibration for a lung measurement can be developed that uses the signal from the 29.2-cm (11-in.) diameter $\mathrm{NaI}(\mathrm{Tl})$ detector centered behind the lungs and may or may not use the output from the other four detectors. The measurement process and counting parameters are otherwise identical to the standup whole body measurement although the count time may be different depending on the particular application. This method is not routine and is a backup to the coaxial HPGe lung measurement protocol described in Section 3.2.2.1.

The result of a standup counter measurement can also be used to obtain an indication of the gross distribution of the activity in the body. The percentage of the total number of counts for each detector is easily determined and provides information that can be used to differentiate lung activity from total body activity. As a rule-ofthumb, if the lung detector contributes greater than $50 \%$ of the total measured net counts and the activity content is significant, an estimate of the lung activity may be calculated based on a lung calibration factor. This result is only an estimate and, due to interference from detector cross-talk, it can have large uncertainties associated with it. Therefore, if significant lung activity is detected on the standup counter, follow-up measurements are usually obtained.

\subsection{Other Organ Monitoring Systems}

The IVRRF staff performs in vivo measurements to estimate the radionuclide activity in different organs and body locations. Measurements of activity in the skeleton, liver, and wounds are not done routinely but are typically performed following a known or suspected intake. The measurement results are used to evaluate the type of radionuclide, the magnitude of the activity, how the material is distributed as it is metabolized, and ultimately to support assessments of the resulting dose. Skeletal activity is usually estimated with measurements made over the head but measurements of the knees, ankles, and wrists may also be used. This type of measurement is most often used to quantify plutonium and americium activity. Liver monitoring is typically performed using two HPGe planar detectors and is done primarily to estimate the plutonium and americium activity. Measurements to quantify activity in the thyroid, wound, and lymph nodes are also performed. The detection systems used to perform the measurements are discussed in this section.

\subsubsection{Skeletal Monitoring}

When radioactive materials like plutonium, americium, and uranium enter the systemic circulation the skeleton is a major deposition site. Skeletal measurements are usually made over the forehead to estimate the amounts of radioactive material in the skeletons of workers. The head is the preferred measurement location since it is readily accessible and less prone to interference from activity in other tissues and organs. The skull is also known to contain a relatively consistent percentage (13-15\%) of the total skeletal activity for americium and plutonium from shortly after deposition to long times after deposition. Measurements over the wrists, knees, and ankles can also be used to estimate skeletal activity. When using joint measurement results, proper calibration 
and interpretation of the results are essential to obtain accurate skeletal activity estimates. This is due in part to the higher initial activity deposition in the trabecular bone in joints compared to the deposition of activity on cortical bone surfaces.

Measurements of radionuclides such as uranium, thorium, americium, and plutonium in the skeleton are typically made using two $38-\mathrm{cm} 2$ planar HPGe detectors. The detectors are positioned on the forehead to measure the skull activity, and for joint counting, they are placed in light contact with the joint. The gain conversion of the detectors is typically $0.25 \mathrm{keV} /$ channel. Skeletal corrections to lung activity estimates can also be performed using the results from this type of measurement. A correction to the lung activity result may be necessary to account for the contribution from the radioactive material that is contained in the thoracic skeleton including the ribs, sternum, clavicles, scapulae and vertebrae. Figure 3.16 depicts the equipment set-up and counting parameters for a low-energy photon head count.

For radionuclides such as $133 \mathrm{Ba}$ and 154Eu that emit high-energy photons (e.g. $>300 \mathrm{keV}$ ), a whole body count can provide a reasonable estimate of the activity in bone if significant activity does not also reside in other organs or soft tissues.

\subsubsection{Liver Measurements}

The liver is the other major deposition site in addition to the skeleton for uranium and transuranic radionuclides that enter the systemic circulation. Typically, two $38 \mathrm{~cm} 2$ planar Ge detectors such as those shown in Figure 3.15 are used for the liver measurements. The detectors are aligned along the lower rib in the lower right quadrant of the torso. Figure 3.17 illustrates the counting parameters and geometry used for liver measurements. If significant activity is present in the lungs, the contribution to the measured count rate over the liver from activity in the lungs will need to be accounted for. Similarly, liver activity will contribute to the count rate measured over the lungs and may need to be accounted for to obtain the best estimate of activity in the lungs. See Section 4.3.4 for the discussion of making corrections to measured count rates from activity in other organs.

\subsubsection{Thyroid Monitoring}

Measurement of iodine in the thyroid, primarily 125I and 131I, is occasionally performed at the IVRRF. The $\mathrm{K}$ x-rays and gamma photons from 125I $(27.2,35 \mathrm{keV}, 27.5 \mathrm{keV})$ and the $131 \mathrm{I}$ gamma rays (e.g. $364 \mathrm{keV}, 285$ $\mathrm{keV}$ ) are detected with the same type of planar HPGe detectors that are used for low energy lung monitoring. A single detector is positioned $10 \mathrm{~cm}$ (3.75 in.) above the cricoid cartilage and calibrated for an energy response of $0.25 \mathrm{keV} /$ channel for measurements of both $125 \mathrm{I}$ and $131 \mathrm{I}$.

\subsection{Wound Monitoring}

Four different types of detectors can be used for wound monitoring including planar HPGe detectors, coaxial HPGe detectors, NaI detectors, and cadmium telluride (CdTe). The type of detector used depends on many factors including the radionuclide(s) of concern, the activity levels, the location, size and depth of the wound, and the photon energies to be measured. The majority of wound measurements are made using planar HPGe for low energy photons or the coaxial HPGe or NaI detector systems for high energy photons.

\subsubsection{Planar HPGe Wound Detectors}

A planar HPGe thin-window detector is the most frequently used detector for low-energy wound monitoring. A single detector is normally used because most wounds encountered at the Hanford Site are small puncture wounds. The detector is positioned directly over the wound in close proximity. The detector is usually covered with a thin plastic sheet to protect it from contamination. If the measured activity is small, further measurements 
are not usually done. If significant activity is detected, follow-up measurements will be performed to better characterize the areal extent and depth of burial of the activity to improve the accuracy of the activity estimate. Measurement of activity in the proximal lymph nodes may also be made. The counting parameters for this measurement are similar to those used in HPGe lung measurements. For large wounds, detailed mapping of the area may be necessary and case-specific calibrations established.

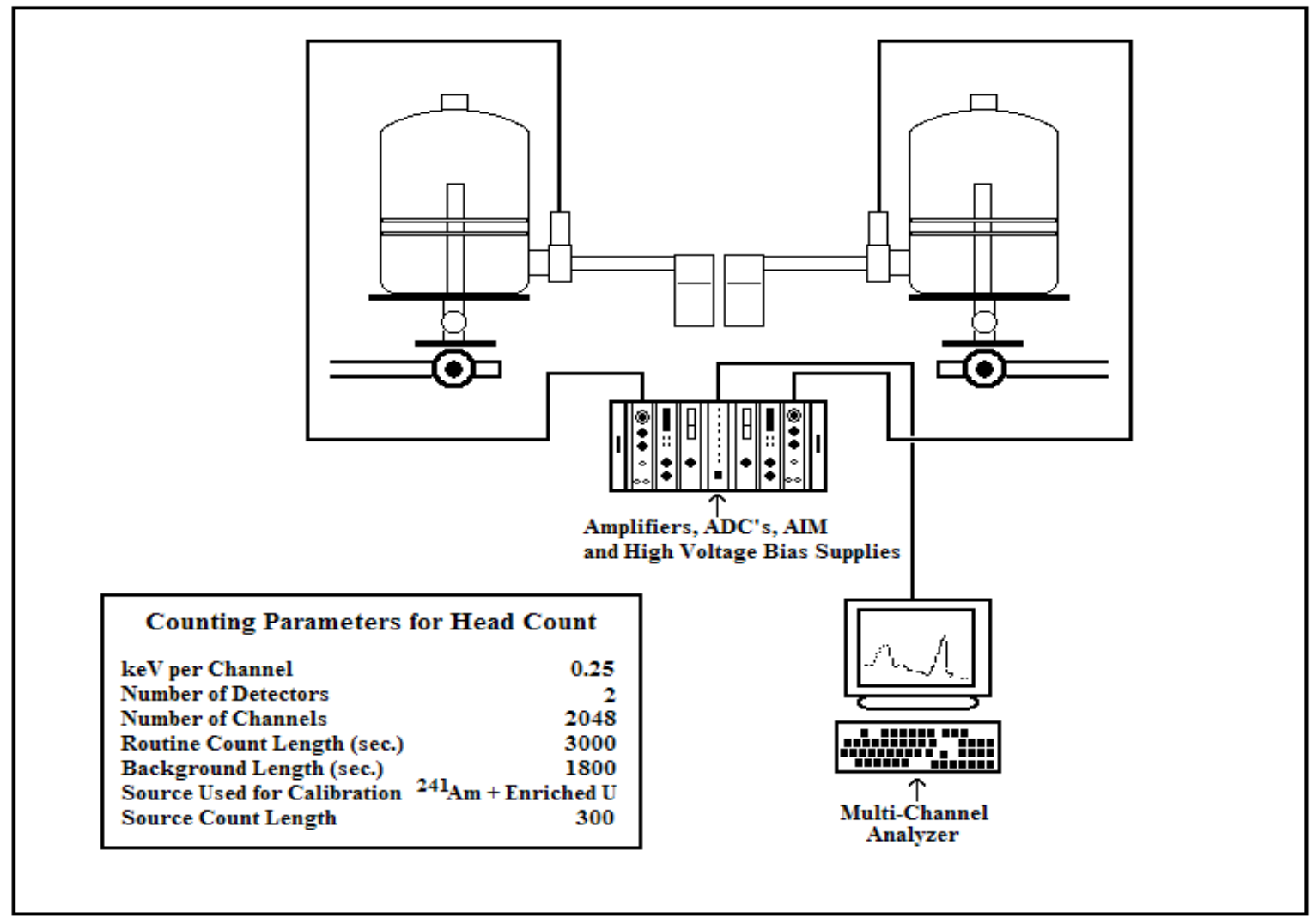

Figure 3.16. Equipment Diagram and Counting Parameters for Low Energy Head Count 

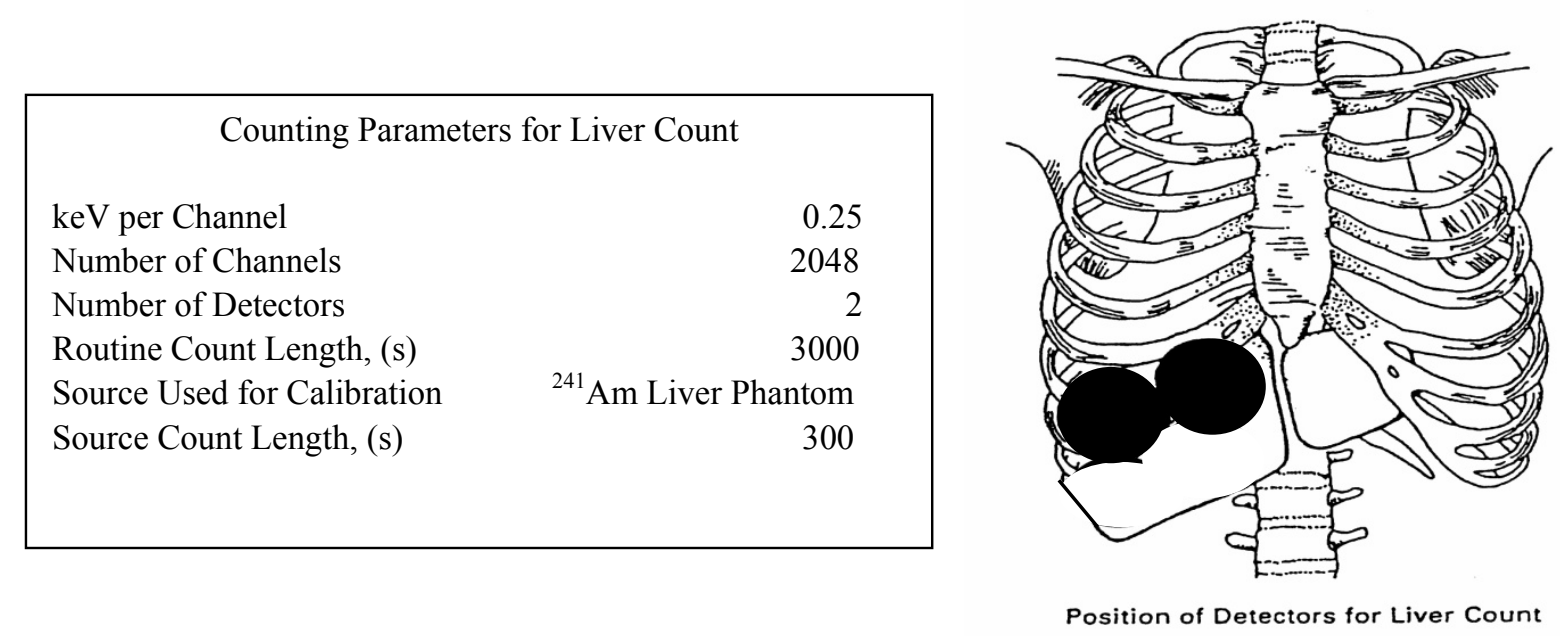

Figure 3.17. Counting Parameters and Counting Geometry for Liver Measurements

A portable wound counting system incorporates a $28-\mathrm{cm}^{2} \times 20-\mathrm{mm}$ germanium detector. The detector is fitted with a 7-liter liquid nitrogen dewar. The dewar has a 5-day hold time. The detector output is directly interfaced to the electronics module. The integrated module contains an MCA that can be powered either by line voltage or by batteries, a spectroscopy amplifier, high-voltage power supply, a digital stabilizer, and an $8192-$ channel analog-to-digital converter. The user interface is provided through the Genie 2000 software that resides on a laptop computer. This system is configured for both high- and low-energy wound counting.

\subsubsection{Other Types of Wound Detectors}

One or more of the NaI detectors from the stand-up counter or coaxial HPGe detectors from the Palmer Room are used to estimate wound activity involving fission and activation products. The CdTe detector could be used to help localize activity in a wound. Due to its small size, the wound activity would need to be at least in the tens to hundreds of nanocuries to be detected with the CdTe measurements.

\subsection{Cited Reference}

Palmer, H. E. and W. C. Roesch. 1965. “A Shadow Shield Whole-Body Counter.” Health Physics 11:12131219.

\subsection{Other Useful References}

Knolls, G. F. 1989. Radiation Detection and Measurement. 2nd ed. J. Wiley and Sons, Inc. New York.

Palmer, H. E. and M. C. Rhoads. 1989. "Determining the Ratio of ${ }^{239} \mathrm{Pu}$ to ${ }^{241} \mathrm{Am}$ in Wounds by Measuring the L $\gamma$ X-Rays with a Si(Li) Detector.” Health Physics 56(2):249-252.

Memorandum, "Technical Equivalency for the Pacific Northwest National Laboratory Radiobioassay Program", November 9, 2009, U.S. Department of Energy, Steven Zobel (DOELAP Administrator) to Ray J. Corey (Richland Operations Office). 
IN VIVO MONITORING PROGRAM MANUAL

$$
\text { PNL-MA-574 }
$$

SECTION 4.0

IN VIVO MEASUREMENT PROTOCOLS 


\section{Contents}

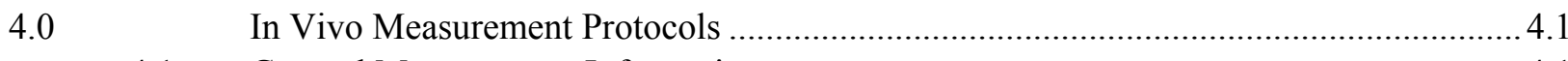

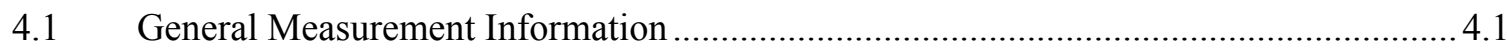

4.2 Whole Body in Vivo Measurements ............................................................................ 4.1

4.2.1 Whole Body in Vivo Measurements Using the Standup Whole Body Counter . 4.3

4.2.2 Estimating the Distribution of Activity in the Body Using the Standup Counter

4.2.3 Mobile Counting Trailer ........................................................................... 4.5

4.2.4 Whole Body Measurements Using the Coaxial Ge Detector Array ................... 4.7

4.3 Thorax Measurements............................................................................................... 4.8

4.3.1 Thorax Measurements of Low-Energy Photons, $<500 \mathrm{keV}$.............................. 4.9

4.3.2 Calibration of Low Energy Thoracic Measurement Systems ............................ 4.10

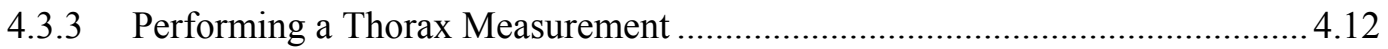

4.3.4 Correction of Organ Activity Estimates for Activity in Other Organs ............. 4.14

4.3.5 In Vivo Lung Measurements of High-Energy Photons, $>500 \mathrm{keV}$................... 4.15

4.4 In Vivo Measurement of Radioactivity in the Skeleton.............................................. 4.17

4.4.1 Calibration and Efficiency Check ................................................................... 4.17

4.4.2 Performance of Skeletal Measurements............................................................. 4.18

4.5 In Vivo Measurement of Radioactivity in the Liver ................................................... 4.18

4.6 In Vivo Measurement of Radionuclides in Wounds ................................................... 4.19

4.6.1 Measurement of Transuranic Radionuclide Activity in Shallow Wounds........ 4.19

4.6.2 Measurement of 239Puand 241 Am in Deep Wounds.................................... 4.20

4.6.3 In Vivo Measurement of Fission and Activation Products in Wounds.............. 4.21

4.7 In Vivo Measurement of Radioactivity in the Thyroid.............................................. 4.21

4.8 In Vivo Measurement of Transuranic Nuclides in Axillary or Cubital Lymph Nodes.. 4.22

4.9 In Vivo Measurement of Transuranic Nuclides in Tracheobronchial Lymph Nodes .... 4.23

4.10 In Vivo Measurement of Radioactivity Using a Linear Scan ....................................... 4.23

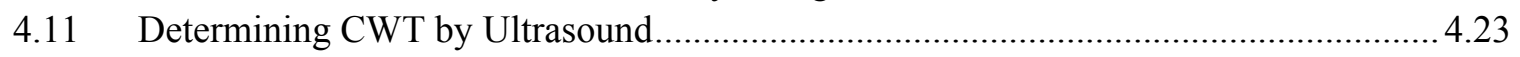

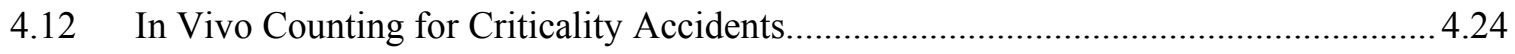

4.12.1 Measuring Activity Resulting from Criticality Accidents ............................... 4.24

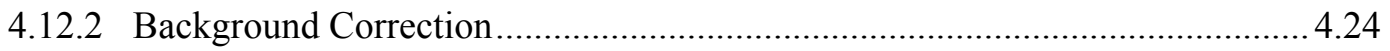

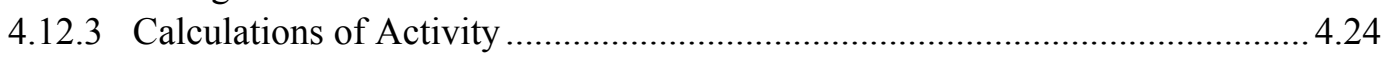

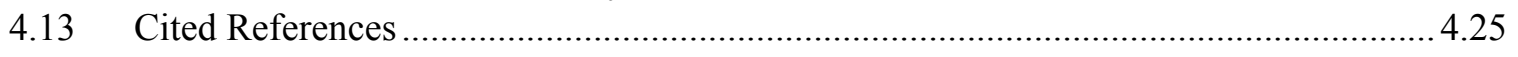

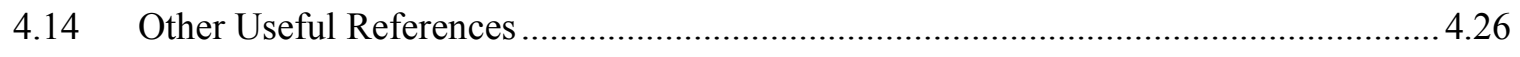




\subsection{In Vivo Measurement Protocols}

This section provides a general description of the in vivo measurement protocols used to detect and quantify radioactive material in the body using the measurement systems described in Chapter 3.0. Sections 4.1 through 4.12 describe whole body, thorax, wound, liver, thyroid, lymph node, and chest thickness measurements. More detailed information on the performance of each type of measurement is contained in the In Vivo Monitoring Program (IVMP) Procedure Manual.

\subsection{General Measurement Information}

As part of the overall worker monitoring program, the purposes of the in vivo measurements are: 1) to identify and quantify internally deposited radioactive material from occupational sources, 2) to document that the estimates of internally deposited radioactive material are below the detection limits of the measurement protocol, and 3) to identify radioactive materials from non-occupational sources.

Section 1.3 describes in general the steps taken to prepare the workers for their measurements. In summary, the workers complete the required paperwork, remove jewelry, watches, etc., may need to change into coveralls, are positioned for the measurement, and receive an explanation about the measurement including safety information. A closed-circuit television system allows the technician to observe the subject in the counting position. Each room also has an intercom system for voice communication. In addition, the workers' height and weight are measured before each measurement.

The results from measurements of radioactive material in the body are currently stated in units of nanocuries.

\subsection{Whole Body in Vivo Measurements}

Whole body in vivo measurements are performed primarily to detect and quantify the amounts of radioactive material that emit high-energy electromagnetic radiation. At Hanford this includes fission products, primarily ${ }^{137} \mathrm{Cs}$, since most activation products have decayed to insignificant levels. However, ${ }^{60} \mathrm{Co}$ and ${ }^{154} \mathrm{Eu}$ are two activation products that are periodically detected. The material may be deposited uniformly or non-uniformly in the tissues and organs of the body. The routine measurement procedures are designed to estimate the activity in the entire body rather than focusing on any one organ or region. However, estimates of organ activities can also be made with the appropriate choice of calibrations and counting geometry. Three different measurement systems are used to perform whole body in vivo measurements: the standup whole body counter, the five-detector array of large-volume coaxial high purity germanium $(\mathrm{Ge})$ detectors, and the shadow shield counter. Workers receiving only a whole body measurement are usually counted in their street clothes initially. Workers receiving both chest and whole body measurements are provided coveralls to be worn during the measurements.

A 10-minute measurement using the coaxial Ge system is the most frequent type of whole body measurement performed at Hanford. These measurements are used primarily to report results for ${ }^{60} \mathrm{Co}$, ${ }^{137} \mathrm{Cs}$, and ${ }^{154} \mathrm{Eu}$. The detection of ${ }^{137} \mathrm{Cs}$ activity is used as an indicator of a potential intake of transuranic radionuclides in some cases. This is done because some Hanford waste streams contain mixtures of predominantly fission product activity with trace amounts of plutonium and other transuranic radionuclides. A 20-minute recount is usually performed if a result exceeds the decision level $\left(\mathrm{L}_{\mathrm{c}}\right)$ value. The coaxial Ge detector system is routinely used to quantify gamma-emitting radionuclides with energies above $200 \mathrm{keV}$ and could be used in some cases for energies down to $100 \mathrm{keV}$. Figure 4.1 displays a flow chart of the routine whole-body examination process. There may be cases when a recount may not 
be performed following a result above the $\mathrm{L}_{\mathrm{c}}$ value. For example, if it is known that the worker had a previous intake.

For persons with a potential for exposure to fission and activation product activity, an initial whole body count is also performed using the standup counter. This is a 200 -second measurement to determine whether activity is present above the $\mathrm{L}_{\mathrm{c}}$ value. If activity other than ${ }^{40} \mathrm{~K}$ is detected in the body, the subject is then requested to shower and change into clean coveralls provided by the IVMP staff. A second standup count is made, and if the result again exceeds the $\mathrm{L}_{\mathrm{c}}$, other than for ${ }^{40} \mathrm{~K}$, the subject is counted with the coaxial Ge detector system. The results from the coaxial Ge measurement are used as the best estimate of the radionuclide activity in the body.

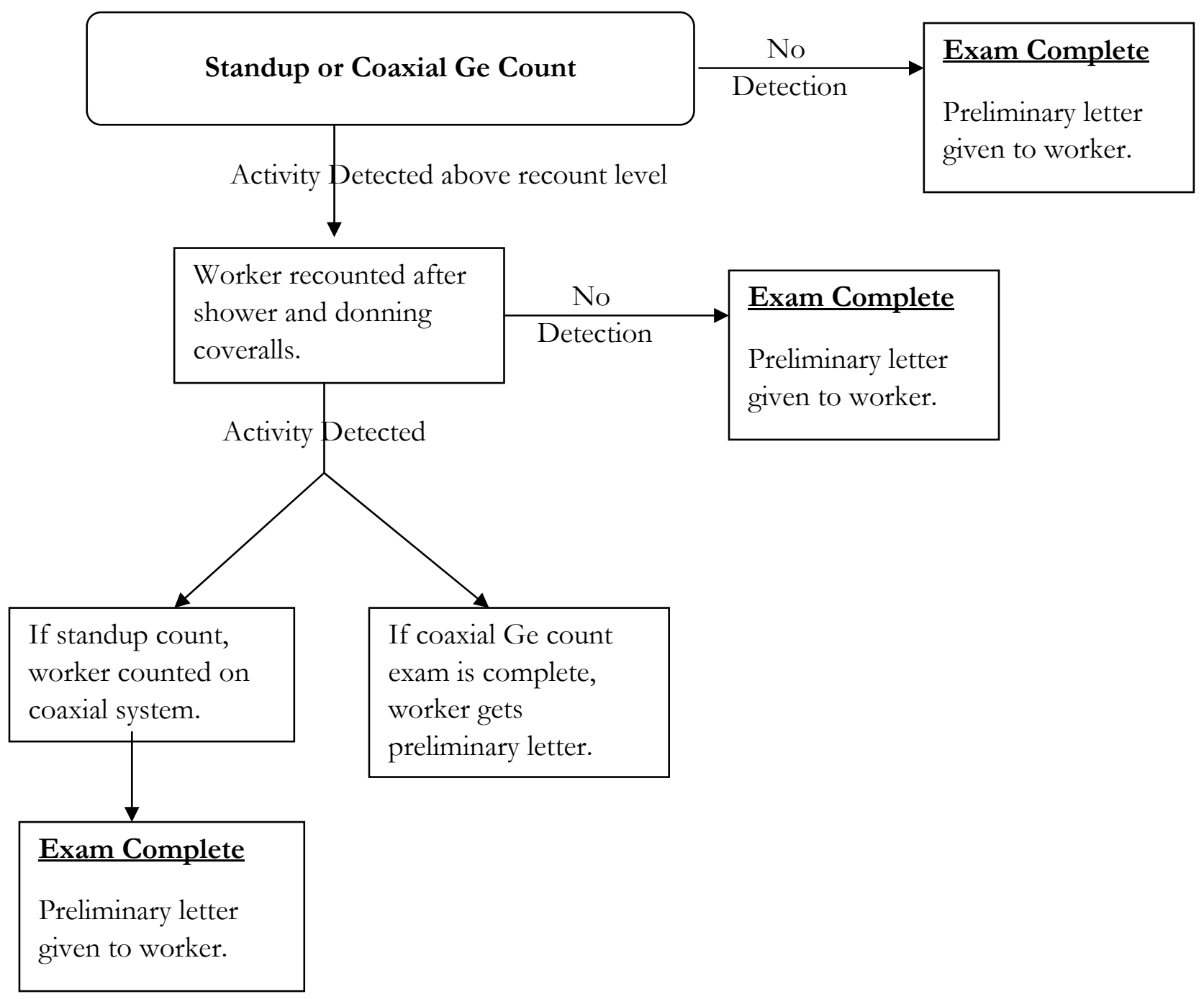

Figure 4.1. General Diagram for Routine Whole Body Examinations 


\subsubsection{Whole Body in Vivo Measurements Using the Standup Whole Body Counter}

Whole body in vivo measurements are performed using the standup counter. This counter consists of a column of five cylindrical thallium-activated sodium iodide (NaI) detectors mounted in a vertical shadow shield arrangement. (See Section 3.1.1 for a more complete description of the standup counting system equipment.) These detectors are used to determine whether detectable quantities of high-energy gamma-emitting radionuclides such as ${ }^{137} \mathrm{Cs},{ }^{60} \mathrm{Co}$, and ${ }^{154} \mathrm{Eu}$ are present in the body.

The subject stands upright in the shielded enclosure. The subject leans back against the slightly inclined Plexiglas sheet that covers the front of the detectors. The five-detector array is then vertically positioned by means of a counter-weighted pulley system. This adjustment accommodates the different worker heights and is used to position the detectors behind the head, chest, abdomen, and legs. Once the detectors are positioned, the count is initiated from the computer console. A 200-second count time is typically used for the routine measurements.

Spectral data from each of the five detectors are summed to provide the composite spectrum that is analyzed with the standard radionuclide library. Table 4.1 lists the radionuclide library parameters that could be used in the analysis of the standup counter result. Currently, results are routinely calculated only for ${ }^{40} \mathrm{~K},{ }^{137} \mathrm{Cs},{ }^{60} \mathrm{Co}$, and ${ }^{154} \mathrm{Eu}$. A plot of each of the five detector regions (head, chest, abdomen, thighs and calves) can also be generated. The relative counts from each detector output provide an approximate measure of the distribution of activity in the body. Table 4.2 presents an example of counting efficiency for lung and whole body activity calculations. The efficiency values based on lung and whole body distributions of activity differ by $10 \%$ or less over the energy range of interest $(600 \mathrm{keV}-1500 \mathrm{keV})$ for routine measurements.

Detection of multiple nuclides can result in interference due to the limited resolution of the NaI detectors and can adversely affect the accuracy of activity estimates. When multiple nuclides are detected, the detection level for nuclides with lower photon energies can be affected due to overlapping energies and interference from Compton scattering of the higher energy photons. The Ge detectors with their significantly better resolution compared to the sodium iodide detectors are not as susceptible to interferences and provide a superior capability for identifying multiple nuclides in a spectrum.

The decision level value $\left(\mathrm{L}_{\mathrm{c}}\right)$ is used to determine if activity is present for all in vivo measurements. The $\mathrm{L}_{\mathrm{c}}$ value is set based on established type I (false positive) and Type II (false negative) error probabilities. A more detailed discussion of the basis for the $\mathrm{L}_{\mathrm{c}}$ values and MDA values for the radionuclides of interest for the standup counter and the other measurement systems is found in Section 7.0. 
Table 4.1. Example Nuclide Library Parameters for Standup Whole Body Counter

\begin{tabular}{cccccc}
\hline & $\begin{array}{c}\text { Energy of } \\
\text { Gamma-ray }\end{array}$ & $\begin{array}{c}\text { Regions-of- } \\
\text { Interest } \\
\text { Channels }\end{array}$ & $\begin{array}{c}\text { Background } \\
\text { Regions-of- } \\
\text { Interest } \\
\text { Channels }\end{array}$ & $\begin{array}{c}\text { Counts per } \\
\text { Minute per } \\
\text { Nanocurie } \\
\text { (cpm/nCi) }\end{array}$ & $\begin{array}{c}\text { Counts per } \\
\text { Minute per } \\
\text { Nanocurie in } \\
\text { the Lung } \\
\text { (cpm/nCi) }\end{array}$ \\
\hline${ }^{40} \mathrm{~K}$ & 1460 & $68-78$ & $79-89$ & 7.4 & 9.1 \\
${ }^{60} \mathrm{Co}$ & 1173 & $54-62$ & $70-78$ & 75 & 84 \\
${ }^{137} \mathrm{Cs}$ & 661.6 & $30-36$ & $38-44$ & 72 & 76 \\
${ }^{54} \mathrm{Mn}$ & 835 & $38-44$ & $45-52$ & 81 & 88 \\
${ }^{24} \mathrm{Na}$ & 2754 & $131-147$ & $148-168$ & 50 & 83 \\
${ }^{22} \mathrm{Na}$ & 511 & $22-28$ & $30-40$ & 160 & 86 \\
${ }^{131} \mathrm{I}$ & 364 & $15-19$ & $20-26$ & 74 & 72 \\
${ }^{208} \mathrm{Tl}$ & 2615 & $125-135$ & $136-147$ & 52 & 16 \\
${ }^{154} \mathrm{Eu}$ & 1274 & $60-68$ & $96-118$ & 10 & 0.7 \\
${ }^{144} \mathrm{Pr}$ & 2186 & $100-120$ & $125-145$ & 0.5 & \\
\hline
\end{tabular}

Table 4.2. Standup Whole Body Counter Efficiency Factors ${ }^{(a)}$

\begin{tabular}{ccc}
\hline $\begin{array}{c}\text { Photon } \\
\text { Energy } \\
\text { (keV) }\end{array}$ & $\begin{array}{c}\text { Total Body } \\
\text { Efficiency } \\
\text { (counts/gamma } \\
\text { emitted) }\end{array}$ & $\begin{array}{c}\text { Lung Counting } \\
\text { Efficiency } \\
\text { (counts/gamma } \\
\text { emitted) }\end{array}$ \\
\hline 100 & $4.40 \mathrm{E}-2$ & $4.08 \mathrm{E}-2$ \\
200 & $4.28 \mathrm{E}-2$ & $4.03 \mathrm{E}-2$ \\
300 & $4.18 \mathrm{E}-2$ & $3.97 \mathrm{E}-2$ \\
400 & $4.07 \mathrm{E}-2$ & $3.92 \mathrm{E}-2$ \\
500 & $3.97 \mathrm{E}-2$ & $3.86 \mathrm{E}-2$ \\
600 & $3.87 \mathrm{E}-2$ & $3.81 \mathrm{E}-2$ \\
700 & $3.77 \mathrm{E}-3$ & $3.76 \mathrm{E}-2$ \\
800 & $3.68 \mathrm{E}-2$ & $3.71 \mathrm{E}-2$ \\
900 & $3.58 \mathrm{E}-2$ & $3.65 \mathrm{E}-2$ \\
1000 & $3.49 \mathrm{E}-2$ & $3.60 \mathrm{E}-2$ \\
1200 & $3.32 \mathrm{E}-2$ & $3.50 \mathrm{E}-2$ \\
1500 & $3.07 \mathrm{E}-2$ & $3.36 \mathrm{E}-2$ \\
2000 & $2.71 \mathrm{E}-2$ & $3.14 \mathrm{E}-2$ \\
2500 & $2.38 \mathrm{E}-2$ & $2.93 \mathrm{E}-2$ \\
3000 & $2.10 \mathrm{E}-2$ & $2.89 \mathrm{E}-2$ \\
\hline (a) Using full photopeak & \\
\hline
\end{tabular}


When results from an initial measurement on the standup counter are less than the $L_{c}$ value no further measurements are necessary. The results are uploaded to the Radiation Exposure System (REX) database. If the initial measurement result exceeds the $\mathrm{L}_{\mathrm{c}}$ value but the results from a second measurement after a shower and change into coveralls are less than the $\mathrm{L}_{\mathrm{c}}$ value, then no additional measurements are performed. The results from the second measurement are retained as the final result and uploaded to REX. A record of the initial measurement is generated without quantitative results and also uploaded to the REX database. If activity is detected on an initial measurement and verified by a second measurement (performed after the subject showers and changes into coveralls), the subject will then be measured on the coaxial Ge system. Quantitative results from the coaxial Ge system are uploaded to REX. Records without quantitative results are also generated for the two initial standup measurements and uploaded to REX. Any confirmed results exceeding the $\mathrm{L}_{c}$ value are reported to the Internal Dosimetry Program (IDP) after the measurements are completed.

\subsubsection{Estimating the Distribution of Activity in the Body Using the Standup Counter}

The distribution of radioactivity in the body can be estimated by analyzing the net peak areas of interest from each of the five individual detector spectra that are collected. This determination is considered a special evaluation and is not regularly performed for each routine count.

The five body regions that correspond to these spectra are as follows:

- Region 1. Head region measured by one $10 \times 23.8 \mathrm{~cm}(3.75 \times 9$ in.) diameter detector,

- Region 2. Chest region measured by one $10 \times 29.2 \mathrm{~cm}(3.75 \times 11$ in.) diameter detector,

- Region 3. Abdomen region measured by one $10 \times 23.8 \mathrm{~cm}(3.75 \times 9$ in.) diameter detector,

- Region 4. Thigh region measured by one 10 x $23.8 \mathrm{~cm}$ (3.75 x 9 in.) diameter detector,

- Region 5. Calf region measured by one $10 \times 23.8 \mathrm{~cm}(3.75 \times 9$ in.) diameter detector.

The five sections of the multichannel analyzer memory are read into the computer for the analysis. Each memory section includes 256 channels of data, representing one of the five general body regions. The relative peak area of each region can be calculated as a percentage of the summed spectrum. This distribution can then be plotted as an overlay plot of all five regions. Figure 4.2 illustrates an example of this plot. The overlay plot provides a visual indication of the relative distribution of activity in the subject.

\subsubsection{Mobile Counting Trailer}

Shadow shield whole body counters can provide sensitive and accurate measurements of radionuclides that emit gamma rays with energies higher than $200 \mathrm{keV}$ in the body (Palmer and Roesch 1965). A horizontal shadow-shield counter and a vertical shadow-shield counter are located in the mobile counting trailer that can be transported to different locations. The trailer also contains additional shielding for organ counting. Various types of detectors are available for installation in the trailer. Table 4.3 contains information on the approximate efficiencies for the horizontal shadow-shield counter. The efficiency values for the vertical shadow-shield counter are approximately $15 \%$ lower than the values shown in Table 4.2 for the standup counter. 


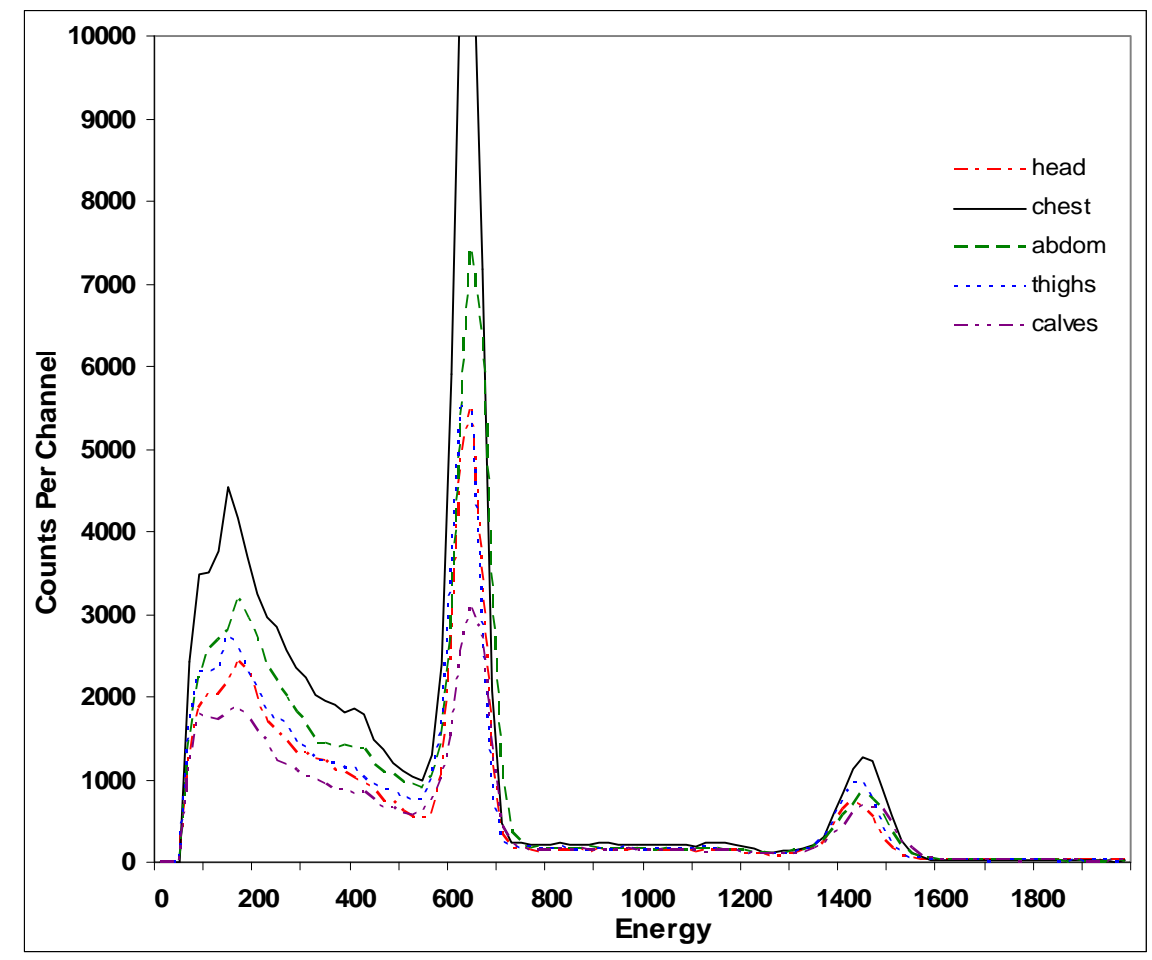

Figure 4.2. An Example Overlay Plot of the Activity Spectra for the Five Body Regions

The shadow shield counters are calibrated using Bottle-Manikin Absorption (BOMAB) phantoms containing ${ }^{137} \mathrm{Cs}$ and ${ }^{40} \mathrm{~K}$. the results from the phantom measurements are used to determine the efficiency (e.g. cpm/nCi) values for the system. Gain conversion settings are usually performed using ${ }^{137} \mathrm{Cs}$ and ${ }^{40} \mathrm{~K}$ check sources.

A measurement on the vertical shadow shield system is similar to a count on the standup counter. The horizontal shadow-shield counter requires the subject to be supine or prone on the sled. During the 1000 -second count, the sled moves the subject under the detector portal, while the detector scans the body. The sled automatically stops at the end of the measurement, and the subject then exits the counter.

The calculation of activity is similar for the vertical shadow shield and the 747A standup counter. The calculation of activity for the horizontal shadow shield counter is performed automatically using a PC-based computer algorithm. For other radionuclides than those listed in Table 4.3, the activity can be determined by using efficiency values from Table 4.2 for the specific photon energy and calculating the activity using Equation (4.1):

$$
\mathrm{nCi} \text { in Total Body }=\frac{\mathrm{C}_{0}}{\mathrm{I} \cdot \mathrm{a} \cdot \mathrm{E} \cdot \mathrm{T} \cdot \frac{\lambda}{\left(1-\mathrm{e}^{-\lambda \mathrm{T}}\right)}}
$$

where $\mathrm{C}_{0}=$ net counts of the measurement

$\mathrm{I}=$ intensity of gamma ray, in gammas per disintegration

$\mathrm{a}=$ conversion factor, $2.22 \mathrm{E}+3 \mathrm{dpm} / \mathrm{nCi}$

$\mathrm{E}=$ counting efficiency, expressed as counts/gamma emitted 


$$
\begin{array}{ll}
\lambda & \mathrm{T}=\text { length of count in minutes } \\
= & \text { decay correction factor for short-lived radionuclides. For } \\
& \text { routine counting, this term is not required because } \\
& \text { counting times are much smaller than the half-lives of } \\
& \text { the nuclides }
\end{array}
$$

Table 4.3. Efficiency and Calibration Data for the Horizontal Shadow-Shield Counter

\begin{tabular}{ccccc}
\hline & & & \multicolumn{2}{c}{$\begin{array}{c}\text { Approximate Efficiency } \\
\text { (cpm/nCi) }\end{array}$} \\
\cline { 4 - 5 } Radionuclide & $\begin{array}{c}\text { Photon } \\
\text { Energy }\end{array}$ & $\begin{array}{c}\text { Peak } \\
\text { Regions-of- }\end{array}$ & $\begin{array}{c}\text { 23.8-cm (9-in.) } \\
\text { Interest }\end{array}$ & $\begin{array}{c}\text { 29.2-cm (11-in.) } \\
\text { Diameter }\end{array}$ \\
\hline${ }^{40} \mathrm{~K}$ & 1460 & $68-78$ & 0.61 & 0.77 \\
${ }^{60} \mathrm{Co}$ & 1173 & $52-62$ & 6.19 & 7.58 \\
${ }^{137} \mathrm{Cs}$ & 661.6 & $30-36$ & 6.24 & 7.33 \\
${ }^{54} \mathrm{Mn}$ & 840 & $38-44$ & 6.95 & 8.26 \\
${ }^{154} \mathrm{Eu}$ & 1274 & $57-68$ & 2.16 & 2.66 \\
${ }^{22} \mathrm{Na}$ & 1275 & $57-68$ & 6.05 & 7.48 \\
${ }^{59} \mathrm{Fe}$ & 1099 & $48-59$ & 3.62 & 4.40 \\
${ }^{95} \mathrm{Zr}$ & 756.7 & $34-42$ & 7.15 & 8.41 \\
${ }^{65} \mathrm{Zn}$ & 1116 & $52-60$ & 3.23 & 3.94 \\
${ }^{106} \mathrm{Ru}-{ }^{106} \mathrm{Rh}$ & 512 & $22-28$ & 1.59 & 1.84 \\
${ }^{131} \mathrm{I}$ & 364.5 & $15-19$ & 6.58 & 7.49 \\
${ }^{140} \mathrm{Ba}-{ }^{140} \mathrm{La}$ & 1596 & $76-84$ & 5.22 & 6.64 \\
${ }^{208} \mathrm{Tl}$ & 2615 & $125-135$ & 1.42 & 1.98 \\
${ }^{214} \mathrm{Bi}$ & 1764 & $83-97$ & 0.97 & 1.25 \\
${ }^{144} \mathrm{Ce}$ & 2180 & $100-120$ & 0.035 & 0.047 \\
${ }^{51} \mathrm{Cr}$ & 320.1 & $13-17$ & 0.81 & 0.916 \\
${ }^{110 \mathrm{~m}} \mathrm{Ag}$ & 658 & $29-35$ & 6.96 & 8.142 \\
${ }^{24} \mathrm{Na}$ & 2754 & $131-147$ & 3.77 & 5.322 \\
\hline
\end{tabular}

These counting systems are maintained in a standby mode but can be readied for operation within a few weeks or so.

\subsubsection{Whole Body Measurements Using the Coaxial Ge Detector Array}

The counting system in the Palmer Room is normally operated with five coaxial Ge detectors. The system has an energy calibration at $0.75 \mathrm{keV} /$ channel over 4096 channels. In the event of detector failure, a calibration with fewer detectors can be used. A computerized motion control system moves the array of five detectors located under the counting platform at one speed from the head to thighs and at a faster speed from the thighs to the lower legs. Ten-minute scanning measurements are routinely performed for workers with a potential for exposure to fission and activation products. Twenty-minute follow-up measurements are made to identify and quantify the radionuclides if a result from the initial measurement exceeded the $\mathrm{L}_{\mathrm{c}}$ value. Some waste streams containing mostly fission product activity can also contain small amounts of transuranic radionuclides. The ${ }^{137} \mathrm{Cs}$ results that exceed the $\mathrm{L}_{\mathrm{c}}$ value from these measurements can provide an indication of a possible plutonium or strontium intake. The plutonium or strontium activity can be estimated using the cesium activity ratio obtained from workplace air samples or smear samples for example. Usually, a work history review, additional in vivo measurements and/or 
excreta samples are obtained to help confirm whether an intake occurred. The ${ }^{137} \mathrm{Cs}$ to plutonium activity ratios in Hanford waste can range from 1000:1 to 1:1. The lower the ${ }^{137} \mathrm{Cs}$ MDA value, the more sensitive the indication for a plutonium intake.

The count times for the measurements can be set to different values as needed. The subject typically lies in a supine position centered on the counting platform. The scan proceeds from the head to the feet and when the scan is completed the detector array is automatically returned to the starting position. Although not performed routinely, stationary whole body measurements can be done where the detector array is positioned under the torso. Upon completion of either a scanning or stationary measurement, the software automatically generates a written report.

Table 4.4 contains an example of the efficiency calibration data determined from scanning measurements of BOMAB phantoms. There is a decrease in efficiency with the larger phantom as seen on the data plot in Figure 4.3. This is due to greater self absorption in the larger phantom and the fact that the effective activity centroid for the larger phantom is farther from the detectors compared to the smaller size phantom. Daily quality control checks are made each day before and again after all workers are counted. The system counting efficiency, energy calibration, and gain stability are checked daily using a ${ }^{152} \mathrm{Eu}$ point source. Equation (4.2) is a simplified expression of the routine algorithm used to estimate the activity in the body.

$$
\text { Activity }=\frac{\text { Gross counts }- \text { Background counts }}{\text { Count time } \times \text { Calibration factor }}
$$

\subsection{Thorax Measurements}

In vivo thorax measurements are designed to quantify activity deposited in the lungs. This type of measurement can be referred to as lung, chest, or thorax measurement. To accurately quantify the activity in the lungs, the contribution from activity in other parts of the respiratory tract, liver, skeleton and perhaps other tissues may need to be accounted for if there has been a measurable intake. The IVRRF has several in vivo measurement systems that are used to measure and quantify both low- and high-energy gamma-emitters in the lungs of subjects. This section describes the general methods used to estimate the radionuclide activity deposited in the lungs. 
Table 4.4. Detection Efficiency versus Energy for Coaxial Ge Scanning System

\begin{tabular}{ccc}
\hline Energy (keV) & $\begin{array}{c}\text { Cts/Gamma } \\
(\mathbf{1 7 0} \text { lb. BOMAB) }\end{array}$ & $\begin{array}{c}\text { Cts/Gamma } \\
\text { (230 lb BOMAB) }\end{array}$ \\
\hline 88 & & 0.00116 \\
122 & 0.00221 & 0.00151 \\
166 & & 0.00175 \\
244 & 0.00268 & \\
344 & 0.00226 & 0.00186 \\
662 & 0.00242 & 0.00177 \\
898 & 0.00214 & \\
964 & 0.00238 & \\
1112 & 0.00228 & 0.00172 \\
1173 & & 0.00168 \\
1332 & & \\
1408 & & \\
1461 & 0.00216 & 0.00158 \\
1836 & 0.0020 & \\
\hline
\end{tabular}

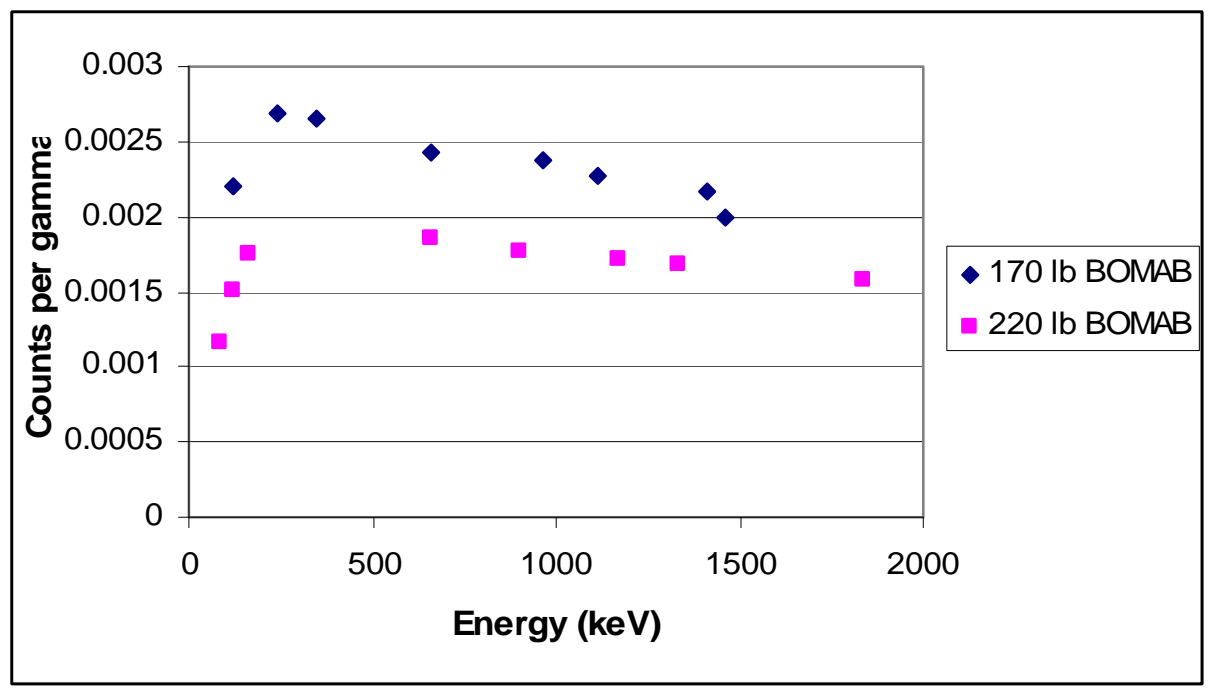

Figure 4.3. Typical Counting Efficiency Plots for 5-Detector Ge Scanning System

\subsubsection{Thorax Measurements of Low-Energy Photons, $<500 \mathrm{keV}$}

The low-energy photons emitted from radionuclides such as uranium, americium, and plutonium that are deposited in the lungs are measured with arrays of four planar Ge detectors. These detectors are placed in light contact with the chest over the lungs with the subject in a semi-reclining position. The left set and right set of detectors straddle the sternum. This provides the highest counting efficiency for both women and men (Palmer 1989). The routine counting time is 3000 seconds. 
The efficiency and hence the MDA value for estimating activity in the lung is strongly affected by the chest wall thickness (CWT) of the subject and any interfering radiations that might be emitted from activity in other parts of the respiratory tract, the skeleton, lymph nodes, soft tissues and liver. The CWT is defined as the thickness of the soft tissue from the exterior surface of the chest to the pleural lining of the lungs. No attempt is made to adjust for thickness or composition of the anterior portion of the thoracic skeleton or the volume or shape of the lungs; it is assumed that the calibration phantom adequately represents the worker. For very large or very small workers this can be a significant source of measurement uncertainty. The subject's CWT is initially estimated using an algorithm based on a heightto-weight ratio. If detection of activity above the Lc value is confirmed, a CWT measurement is made using a diagnostic ultrasound examination. The ultrasonically determined CWT provides a better estimate of the overlying tissue thickness and improves the accuracy of the activity estimate. If direct measurements of the plutonium L-X-rays are made, the relative proportions of the worker's fat and muscle tissue becomes much more important in order to obtain a proper calibration factor. However, for measurements of the $59.5 \mathrm{keV}$ photon from ${ }^{241} \mathrm{Am}$ and higher energies there is only a few percent difference in transmission through fat and muscle so the determination of the relative percentages is not as critical for routine measurements.

The measurement of plutonium isotopes in the lung using the direct $\mathrm{x}$-ray emissions from plutonium is not currently performed on a routine basis. An increased sensitivity for the mixture of plutonium isotopes normally found at Hanford has been developed and used for many years by measuring the 59.5 $\mathrm{keV}$ gamma photon from the ${ }^{241} \mathrm{Am}$ progeny of ${ }^{241} \mathrm{Pu}$. The ${ }^{241} \mathrm{Am}$ MDA for an average CWT can be 1000 times lower than the MDA for ${ }^{239} \mathrm{Pu}$ measured directly (e.g., $0.2 \mathrm{nCi}$ versus $200 \mathrm{nCi}$ ). By measuring the

${ }^{241} \mathrm{Am}$ gamma ray and estimating the $\mathrm{Pu} / \mathrm{Am}$ ratio, the corresponding ${ }^{239} \mathrm{Pu}$ MDA value is obtained. Other factors that complicate the direct measurement of plutonium $\mathrm{x}$-ray emissions include electronic and microphonic noise which can intermittently disrupt the low-energy spectral region, greater sources of error in the activity determination due to CWT measurement uncertainty, higher false-positive rates from interferences, and greater statistical uncertainty in the measured count rates due to greater attenuation in the body and smaller photon abundances of the plutonium L X-rays.

By using the ratio of plutonium isotopes and the "age" of the plutonium since it was separated, an estimate of plutonium activity in the lungs and other organs can be made from the ${ }^{241} \mathrm{Am}$ results. This methodology assumes that the biodistribution and translocation of the americium is the same as the plutonium. If the biokinetics are different, there may be a bias introduced into the plutonium activity estimate. Excreta samples are also collected for workers on a plutonium bioassay schedule and are also collected in cases of confirmed intakes. The excreta analysis results and the in vivo results are factored into the dose assessment process.

\subsubsection{Calibration of Low Energy Thoracic Measurement Systems}

The thorax monitoring systems are housed in separate shielded rooms. An array of planar Ge detectors is located in the Iron Room, Lead Room and Stainless Steel Room. (See Sections 2.0 and 3.0 for a discussion of the rooms and equipment.)

The primary efficiency calibration is based on measurements of lung phantoms placed in a torso phantom. The phantom was originally designed and fabricated by the Lawrence Livermore National Laboratory (LLNL) and the phantom is often referred to as the LLNL torso phantom. Three sets of chest overlays are available for use with the phantom to develop an efficiency calibration as a function of CWT. The three sets have tissue-substitute properties that simulate: 1) 100\% International Commission on Radiological Units and Measurements (ICRU) muscle, 2) 50\% ICRU muscle - 50\% ICRU fat, and 3) $87 \%$ fat $-13 \%$ muscle. The $87 \%-13 \%$ overlay set has historically been used and continues to be used for the Hanford workers. As mentioned in Section 4.3.1 there is only a few percent difference in 
transmission through fat or muscle for energies $\geq 59.5 \mathrm{keV}$. Lung phantoms used to calibrate the thorax monitoring systems contain ${ }^{241} \mathrm{Am},{ }^{234} \mathrm{Th}$ or ${ }^{235} \mathrm{U}$ activity uniformly distributed in the lung phantom made from tissue substitute plastic. The lung phantoms are inserted into the torso phantom. Table 4.5 provides typical calibration data for the Ge lung counter arrays. The data are plotted in Figure 4.4.

Calibration curves are developed that depict the detection efficiency for a given energy as a function of the chest-wall thickness. Figure 4.5 illustrates the efficiency as a function of chestwall thickness and energy. Nuclide specific calibration factors are developed for the three radionuclides of dosimetric concern at Hanford. A calibration is specific to each of the lung-counting systems. The calibration factors are applied to the net count rate to quantify the activity of transuranic and other low-energy gamma-emitting radionuclides in lung. The phantom is also used to generate a similar type of calibration for liver measurements. The phantom can also be used to develop efficiency calibrations as needed for estimating the activity in tracheobronchial lymph nodes.

The gain conversion, efficiency, and resolution for the systems are checked daily using ${ }^{241} \mathrm{Am}$ and ${ }^{152} \mathrm{Eu}$ sources. The efficiency values are the primary parameters used to determine whether the system performance is within the established control chart limits. More variability is tolerated in the individual detector performance if the summed configuration is within the control chart limits. If the centroid channel falls outside of the operating tolerances for the sum configuration, it can affect the efficiency results as well as the resolution results. Consequently, the gain may require readjustment as necessary for the affected detectors unless the count was performed at the end of the day. For the end-of-day measurements the efficiency check is rerun. Results outside control chart limits require investigation to determine whether the worker counts that day were valid. The quality control (QC) data is analyzed periodically to identify long term performance trends.

Detailed procedures on setting the gain conversion, and developing the efficiency, energy and full width at half maximum (FWHM) calibrations for the lung monitoring systems are found in the IVMP Procedure Manual, PNL-MA-554.

Table 4.5. Example Calibration Factors for Lung Counting Systems

\begin{tabular}{cccc}
\hline $\begin{array}{c}\mathbf{C W T} \\
\mathbf{( c m )}\end{array}$ & $\begin{array}{c}\mathbf{A m - 2 4 1} \\
\mathbf{5 9 . 5} \mathbf{~ k e V}^{(\mathbf{a})}\end{array}$ & $\begin{array}{c}\mathbf{T h}-234 \\
\mathbf{6 3 . 3} \mathbf{~ k e V}^{(\mathbf{a})}\end{array}$ & $\begin{array}{c}\mathbf{U}-235 \\
\mathbf{1 8 5 . 7} \mathbf{k e V}^{(\mathbf{a})}\end{array}$ \\
\hline 1.62 & 0.017 & 0.017 & 0.019 \\
2.31 & 0.013 & 0.014 & 0.015 \\
2.90 & 0.011 & 0.011 & 0.013 \\
3.42 & 0.009 & 0.009 & 0.011 \\
4.11 & 0.007 & 0.008 & 0.010 \\
5.39 & 0.005 & 0.005 & 0.007 \\
\hline
\end{tabular}




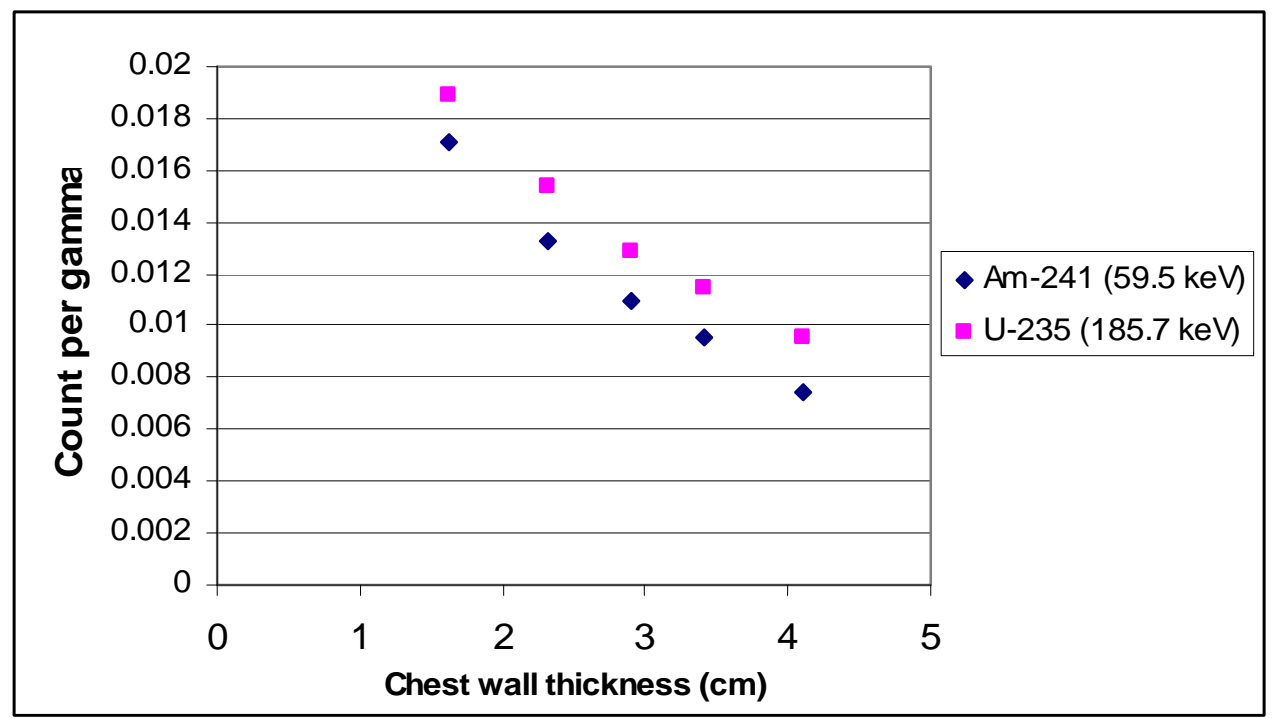

Figure 4.4. Efficiency Curve as a Function of CWT

\subsubsection{Performing a Thorax Measurement}

\subsubsection{Procedure for Thorax Counting}

The subject prepares for the count by changing into clean cotton coveralls and foot covers. This helps minimize migration of external contamination into the counting rooms. Once the subject is brought into the counting room, they are positioned so the detectors are in light contact with the chest. After the subject is briefed on the counting procedure, the shielded door is closed and count acquisition is initiated. When acquisition has completed, the sum spectrum is analyzed and a report is printed. The spectra and calculated results are saved on the computer disk. A result sheet is printed, reviewed, and retained as a program record. 


\section{Efficiency as a Function of CWT and Energy}

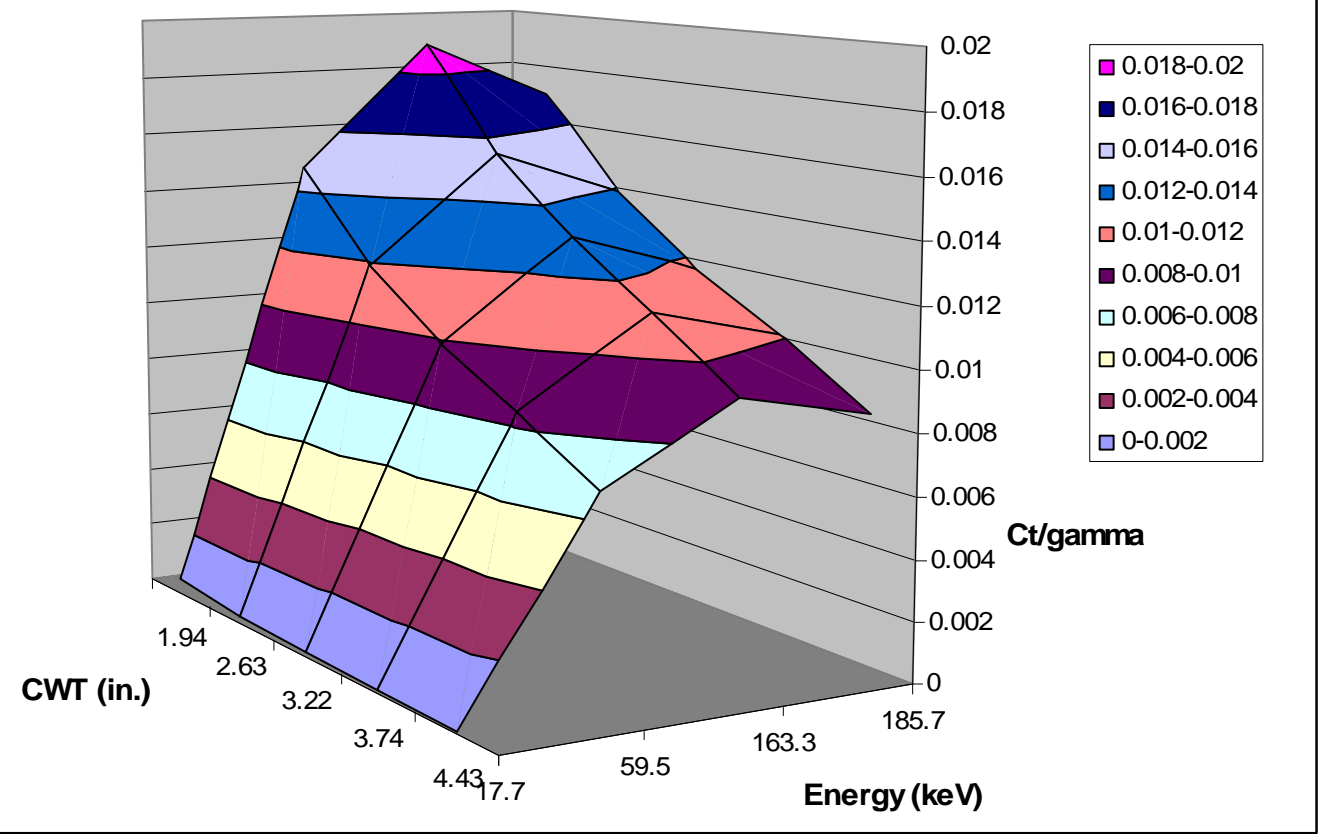

Figure 4.5. Lung Efficiency Calibration Curve

A result exceeding the $L_{c}$ value from a routine measurement usually necessitates further measurements to confirm whether activity is present. If activity is confirmed, an ultrasound exam may be performed to better estimate the worker's CWT to provide a more accurate calibration factor for quantifying activity.

\subsubsection{Estimation of CWT}

An initial estimate of CWT in centimeters is calculated from the subject's height and weight using Equation (4.3).

$$
\mathrm{CWT}(\mathrm{cm})=0.68+\left(0.974 \cdot \frac{\mathrm{W}}{\mathrm{H}}\right)
$$

where $\mathrm{W}$ is the subject's weight in pounds and $\mathrm{H}$ is the subject's height in inches.

See Section 4.11 for additional details on chest wall thickness ultrasound measurements. If the count results are less than the $\mathrm{L}_{\mathrm{c}}$ value, the calculated CWT value obtained using Equation 4.3 is used in the activity calculation. However, if the count result exceeds the $\mathrm{L}_{c}$ value an ultrasound CWT measurement may be performed and the results recalculated using the measured value. The CWT estimates calculated with Equation 4.3 and results based on ultrasound measurements usually agree within $30 \%$ but larger differences have been observed.

Equation (4.3) was developed from CWT data determined by ultrasonic measurements and related to the worker's height and weight. It is specific to Hanford workers. A linear regression curve was 
developed to describe the relationship between ultrasonically measured CWT and the weight to height ratio. Figure 4.6 illustrates the CWT prediction equation.

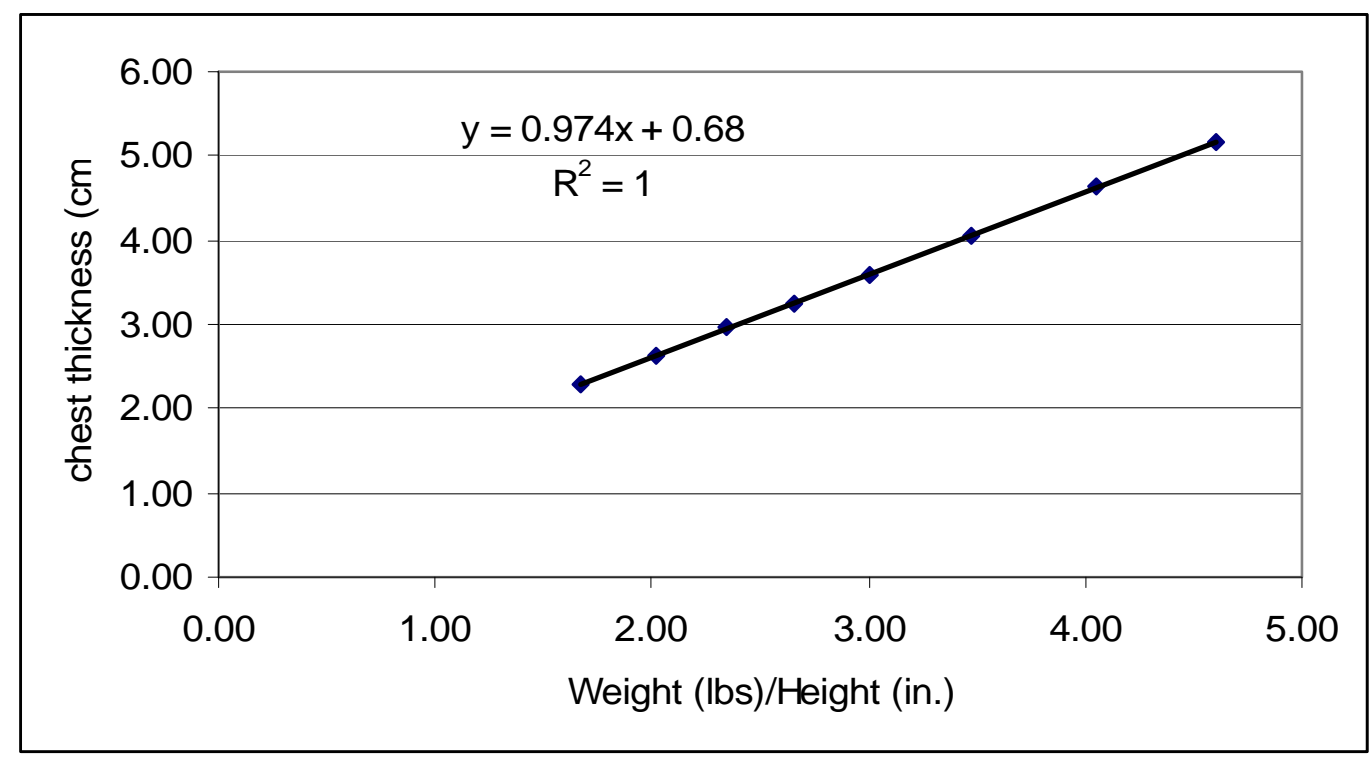

Figure 4.6. Chest Thickness Predication Equation

\subsubsection{Correction of Organ Activity Estimates for Activity in Other Organs}

When transuranic elements such as ${ }^{241} \mathrm{Am}$ and ${ }^{239} \mathrm{Pu}$ are deposited in the lung, a certain amount of the radioactive material will translocate to other organs, notably the liver and skeleton, depending on the solubility of the material. When significant activity is present in multiple organs, it may be necessary to make corrections to the specific organ activity estimates to account for contributions (i.e., cross-talk) to the measured count rate from the activity in adjacent organs. A $3 \times 3$ efficiency calibration matrix is available to make the adjustments necessary to provide the best estimate of the activity in the individual organs (Neton, 1988). This type of correction is applied when the measured activities are typically at or above the MDA. At lower activities the measured count rate over the organ is used to provide the activity estimate. This may provide a conservative activity estimate if detectable activity levels are present in the non-target organs. The contribution from activity in the liver and lung to the head count used to estimate skeletal activity is typically small on the order of 0.05 counts per minute over the forehead per $\mathrm{nCi}$ in the lungs and 0.01 counts per minute over the forehead per $\mathrm{nCi}$ in the liver. Equations (4.4) through (4.7) illustrate how the corrections are made.

Equation (4.4) is used to correct a chest count for activity in the skeleton:

$$
\mathrm{cpm}_{\text {lung }}=\mathrm{cpm}_{\text {chest }}-\left(\mathrm{ratio}_{\text {lung } / \mathrm{skel}} \cdot \mathrm{cpm}_{\text {skel }}\right)
$$

where $\quad \mathrm{cpm}_{\text {chest }}=$ count rate from subject's chest measurement ratio $_{\text {lung/skel }}=$ ratio of count rate over the lungs to count rate over head of the ${ }^{241}$ Am skeletal phantom

$\mathrm{cpm}_{\text {skel }}=$ count rate from subject's head measurement

Equation (4.5) is used to correct a chest count for activity in the liver: 


$$
c p m_{\text {chest (liver })}=c p m_{\text {chest }}-\left(\text { ratio }_{\text {chest /liver }} \cdot c p m_{\text {liver }}\right)
$$

where $\quad \mathrm{cpm}_{\text {chest }}=$ result from chest measurement

ratio $_{\text {lung/iver }}=$ ratio of count rate measured over the lungs to the count rate measured over the liver with only liver activity in the phantom

$\mathrm{cpm}_{\text {liver }}=$ count rate from subject's liver measurement corrected for skeletal activity

Equation (4.6) is used to correct a liver count for activity in the skeleton:

$$
\mathrm{cpm}_{\text {liver }}=\mathrm{cpm}_{\text {abdomen }}-\left(\mathrm{ratio}_{\text {liver/skel }} \cdot \mathrm{cpm}_{\text {skel }}\right)
$$

where $\mathrm{cpm}_{\mathrm{abdomen}}=$ count rate over liver ratio $_{\text {liver/skel }}=$ ratio of count rate over the liver

to count over the head of ${ }^{241}$ Am skeletal phantom $\quad \mathrm{cpm}_{\text {skel }}=$ count rate from subject's head measurement

Equation (4.7) is used to correct a liver count for activity in the lung:

$$
\mathrm{cpm}_{\text {liver/lung }}=\mathrm{cpm}_{\text {abdomen }}-\left(\mathrm{ratio}_{\text {liver/lung }} \cdot \mathrm{cpm}_{\text {lung }}\right)
$$

where $\mathrm{cpm}_{\mathrm{abdomen}}=$ count rate over liver ratio $_{\text {liver/lung }}=$ ratio of count rate over the liver to the count rate over the lungs with only lung activity in the torso phantom $\mathrm{cpm}_{\text {lung }}=$ count rate from subject's chest count corrected for skeletal activity

\subsubsection{In Vivo Lung Measurements of High-Energy Photons, $>500 \mathrm{keV}$}

The measurement of high-energy photons from radioactive material in the lungs is also performed at the IVRRF. Detection of fission and activation products by whole body measurements in the range of a few $\mathrm{nCi}$ are based on a whole body distribution. However, for significantly higher activities an estimate of the lung activity may be warranted. Examples of methods that can be used for this purpose are discussed below. The routine method involves the use of the coaxial Ge system in the Palmer Room. Recalibrating a lung monitoring system or using the standup counter are other alternatives that require additional setup time.

\subsubsection{Coaxial Ge Lung Monitoring}

Measurements to estimate lung activity using the coaxial Ge system can be performed with the system configured for scanning or stationary measurements. (See Sections 3.2.2.1 and 4.3.5 for more detailed information.) The variable velocity scanning measurement has proven to be capable of quantifying lung activity in a torso phantom within $10 \%$ using a whole body calibration based on BOMAB phantom measurements. Except in special cases, the results from the scanning measurements are used to quantify activity in the lungs and whole body.

For increased efficiency, the scanning mechanism can be deactivated so that a measurement is performed with the detectors in a stationary mode. The measurement is set up by placing the subject in a 
supine position on the counting platform in the Palmer Room. This is similar to setting up a subject for the routine scanning measurement. In this case, however, the motion control system is deactivated for the stationary measurement geometry. The detectors are positioned as a group directly beneath the subject's chest area, so that the center of the active area of the detector crystals is underneath the lungs.

The energy calibration is typically $0.75 \mathrm{keV}$ per channel over 4096 channels and all other parameters except the efficiency calibration remain the same as the coaxial Ge scanning measurement. The calibration is based on measurements of lung phantoms in a torso phantom with simulated scapulae in a posterior counting geometry.

\subsubsection{Recalibration of the Low-Energy Lung Monitoring Systems}

Normally, the operational energy range for the lung monitoring systems is $15 \mathrm{keV}$ to $500 \mathrm{keV}$. The energy calibration for the systems is set to $0.25 \mathrm{keV}$ per channel for the 2048 -channel spectra. In order to use one of the planar Ge systems for measurement of high-energy photons, a change in the gain conversion and/or the number of channels in the spectrum is necessary. The $38-\mathrm{cm}^{2} \times 30 \mathrm{~mm}$ thick crystals will provide adequate detection efficiency for high-energy photons for a specified count time.

This method of measurement is not a routinely performed procedure and, as such, does not have an operating procedure associated with it. This would take some time to reconfigure the system and complete a calibration. The steps in completing a gain conversion change are summarized here:

1. Calibration - The amplifier gain must be reduced to diminish the amplitude of the input signal from the preamplifier. A gain conversion of $1 \mathrm{keV}$ per channel over 2048 channels covers the energy range up to $2 \mathrm{MeV}$. Using a calibration lung set (e.g. ${ }^{152} \mathrm{Eu}$ ), the selected high energy peaks are set at their proper channel. An efficiency calibration is then developed for the particular radionuclide of interest, or a calibration equation is developed for the range of photon energies that are under study. Chest wall thickness calibration curves may also need to be developed to account for the effects of the chest wall thickness. Control charts will also be generated to monitor the system performance.

2. Measurement - The subject can now be measured using the planar Ge array in its reconfigured mode. The placement of detectors for this type of lung count is identical to that used for low-energy photons. At the completion of the measurement, a peak search of the entire spectrum is performed and the net count rates are calculated. The activity is calculated by a computer or manually using Equation (4.8).

$$
\begin{aligned}
\text { nCi in lungs }= & \frac{\mathrm{C}_{0}}{\mathrm{I} \cdot \mathrm{a} \cdot \mathrm{E} \cdot \mathrm{T} \cdot \frac{\lambda}{\left(1-\mathrm{e}^{-\lambda \mathrm{T}}\right)}} \\
\text { where } \mathrm{C}_{0}= & \text { net counts of the measurement } \\
\mathrm{a}= & \text { conversion factor, } 2.22 \mathrm{E}+3 \mathrm{dpm} / \mathrm{nCi} \\
\mathrm{E}= & \text { lung counting efficiency, expressed as } \\
& \text { counts/gamma emitted } \\
\mathrm{T}= & \text { length of count in minutes } \\
\mathrm{I}= & \text { intensity of photon in gammas/disintegration } \\
= & \text { decay correction factor for short-lived } \\
\lambda \quad & \text { radionuclides. For routine counting this factor is } \\
& \text { not needed, the half-lives of nuclides at Hanford } \\
& \text { are much longer than the counting times }
\end{aligned}
$$




\subsubsection{Standup Counter Lung Monitoring}

A standup counter measurement can also be used to estimate activity in the lung. The detectors are positioned with the largest detector behind the lungs. Lung activity is estimated by applying an appropriate lung calibration factor to the net counts calculated for the entire array or for the lung detector's photopeak only. A rule of thumb is if $50 \%$ or more of the net counts in the photopeak of a sum spectrum are attributed to the large detector behind the lungs, then it is likely that the activity is in the lungs.

\subsection{In Vivo Measurement of Radioactivity in the Skeleton}

Certain radionuclides are known to deposit in the skeleton after entering the systemic circulation. These include plutonium, americium, uranium, barium, and europium. For radionuclides emitting highenergy gamma rays $>100 \mathrm{keV}$ (e.g., ${ }^{154} \mathrm{Eu}$ ), a whole body count provides an adequate measurement of the activity in the skeleton and soft tissues. For low-energy emitters, special measurements must be performed to properly quantify the skeletal activity. At present, most skeletal measurements are performed to measure low-energy $\mathrm{x}$-rays or gamma rays with energies $<100 \mathrm{keV}$.

Estimates of plutonium and americium activity in the skeleton are based on measurements made with detectors positioned adjacent to the head. Measurements over joints can also be used to estimate skeletal activity. The skull is known to contain a relatively constant percentage of the total skeletal content for transuranic materials. The skull including the mandible was found to contain $13 \%$ of the fresh skeletal mass in 20 adult Russians of normal build (ICRP 2002). An average of $13 \%$ of the total skeletal ${ }^{241} \mathrm{Am}$ activity was found in the skull plus the mandible for both ${ }^{241} \mathrm{Am}$ and ${ }^{239} \mathrm{Pu}$ in four whole body donations to the USTUR (Lynch 1988). The 13\% value is used to extrapolate the results from head measurements to skeletal activity. Since the USTUR data represents the skeletal activity 25 to 35 years after the intake, the use of the $13 \%$ value for shorter times after intake must be used with due consideration since the relative skeletal distribution can change with time. Initial skeletal deposition of transuranic radionuclides in the skeleton is higher on trabecular bone surfaces. Subsequent bone remodeling will redistribute the activity more evenly between the cortical and trabecular bone surfaces. Nonhuman primate studies have shown that the percentage of the total skeletal plutonium activity in the skull including mandible ranged from $7 \%$ to $12 \%$ from 67 to 1021 days after injection of ${ }^{238} \mathrm{Pu}$ citrate (Lynch 1989).

Skeletal activity is estimated by measuring the activity content in the skull with two planar Ge detectors positioned over the forehead. The head is the preferred location for a skeletal measurement because of the small thickness of overlying tissue, the relatively constant percentage of total transuranic skeletal activity, and the smaller potential for interference from activity in other parts of the body. However, other parts of the body such as the knee, ankle, or wrist joints may be measured if the head has extensive contamination (Palmer, Rieksts, and Icayan 1983) or otherwise cannot be used reliably.

\subsubsection{Calibration and Efficiency Check}

The energy range for the calibration extends from $17 \mathrm{keV}$ to $444 \mathrm{keV}$. The efficiency values are determined from measurements of two skull phantoms. One phantom contains only ${ }^{241} \mathrm{Am}$ activity and the other phantom contains ${ }^{241} \mathrm{Am}$ and ${ }^{152} \mathrm{Eu}$. The gain conversion for the planar Ge detectors is typically set to $0.25 \mathrm{keV} /$ channel over 2048 channels using a source containing ${ }^{241} \mathrm{Am}$ and ${ }^{152} \mathrm{Eu}$. This is the standard energy calibration for this system, so no adjustment is necessary from the routine lung counting operations. 
The efficiency check is performed with the ${ }^{241} \mathrm{Am}$ and ${ }^{152} \mathrm{Eu}$ point sources as part of the daily QC check for the lung counter. Figure 4.7 displays a plot of the calibration data for the head count using two $38-\mathrm{cm}^{2}$ planar Ge detectors. The software algorithm calculates the net count rate in the energy regions of interest (ROI), and the net count rate is divided by the appropriate calibration factor to estimate the skeletal activity. The calibration factor is usually expressed as the count rate measured over the head per unit activity in the skull including mandible. The skeletal activity is then calculated based on the skull containing $13 \%$ of the total skeletal activity.

\subsubsection{Performance of Skeletal Measurements}

The subject dons coveralls and may be asked to shower before the skeletal measurement. The skeletal count is performed in a shielded room with a typical count time of 3000 seconds. The skeletal measurement is normally performed with two Ge detectors placed in light contact with the forehead. The results are automatically calculated by the software to provide an estimate of the skull activity. If measurements are made of the activity in the joints, an appropriate efficiency calibration is established to estimate the skeletal activity based on the joint used and the number of detectors.

\subsection{In Vivo Measurement of Radioactivity in the Liver}

Radionuclides such as americium and plutonium and the rare earth elements have a preferential uptake in the liver as well as the skeleton after they are absorbed into the bloodstream. Liver measurements are normally made using two $38 \mathrm{~cm}^{2} \mathrm{Ge}$ planar detectors positioned over the liver.

Measurements of liver activity are typically made to quantify ${ }^{241}$ Am activity, but the same measurement procedures can be used along with a proper efficiency calibration for other radionuclides with photon energies $<500 \mathrm{keV}$. Table 4.6 provides calibration information for the ${ }^{241} \mathrm{Am}$ liver measurements. Calibration is performed using an anthropometric liver phantom placed in an anthropometric torso phantom.

The detectors are positioned for a liver measurement by aligning the tops of the two detectors along the lower rib on the right side of the subject. The detectors are placed so the entrance windows are in light contact with the body.

The liver measurement is performed in a shielded room, normally with a count length of 3000 seconds. The result is provided by automated computer analysis. If activity is also present in the skeleton and lungs, the liver count rate may need to be adjusted for contributions from activity in those organs.

Table 4.6. Example of Calibration Data for Liver Measurements of ${ }^{241} \mathrm{Am}$ Using Four $38-\mathrm{cm}^{2} \mathrm{Ge}$ Detectors

\begin{tabular}{|c|c|c|}
\hline $\begin{array}{l}\text { Radionuclide and } \\
\text { Photon Energy }\end{array}$ & $\begin{array}{l}\text { Regions-of-Interest } \\
\text { (Channels) }\end{array}$ & $\begin{array}{c}\text { cps/nCi } i^{(\mathrm{a})} \text { in the } \\
\text { Liver }\end{array}$ \\
\hline${ }^{241} \mathrm{Am}(59.5)$ & $234-242$ & 0.105 \\
\hline
\end{tabular}

For ${ }^{241} \mathrm{Am}$, the bone contribution to the liver count rate is approximately $30 \%$ of the head count rate and the lung activity contribution to the liver count rate is approximately $5 \%$ of the measured lung count rate. 


\subsection{In Vivo Measurement of Radionuclides in Wounds}

Historically at Hanford, contaminated injuries have generally involved puncture wounds, abrasions, and small cuts to the extremities and head. These cases can result in the injection of radioactive material into the skin and underlying tissue. The wound measurements provide an initial indicatation whether activity is present and at what level. This information helps estimate how much activity may be expected to enter the systemic circulation and lymph system. For dosimetrically significant activities, the measurement results are used to help determine the course of treatment. Accurate estimates of the activity in wounds that are contaminated with transuranic or other nuclides that emit low-energy photons may require an estimate to be made of the burial depth in the wound. Methods are available to estimate the depth of the wound activity by measuring the transmission of different energy photons through thin layers of plastic or paraffin.

\subsubsection{Measurement of Transuranic Radionuclide Activity in Shallow Wounds}

Historically at Hanford, most wounds involving contamination with transuranic activity have been superficial wounds. The activity in these types of wounds is measured with a single Ge detector in light contact with the skin. The detector face is protected with a thin piece of plastic to preclude contaminating the detector. The initial estimate of activity is based on a calibration factor determined from measurements with the detector in light contact with the calibration source. If significant activity levels are detected in a deep wound, a more suitable calibration factor would be determined to better estimate the activity.

\subsubsection{Subject Measurement}

The detector is placed directly on or just over the wound site. The detector's entrance window is covered with a thin plastic layer to protect it from contamination. Only light pressure if any is applied to the subject's wound. The count length is typically 600 seconds and is performed in a shielded room. For $17 \mathrm{keV}<\mathrm{E} \leq 186 \mathrm{keV}$, a typical efficiency calibration for a single $38-\mathrm{cm}^{2} \mathrm{Ge}$ detector is shown as Equation (4.9):

$$
\begin{aligned}
& \text { Efficiency }(\text { counts/gamma })=3 E-07 \mathrm{x}^{3}-0.0001 \mathrm{x}^{2}+0.0095 \mathrm{x}+0.1577 \\
& \text { where } \mathrm{x}=\text { energy in } \mathrm{keV}
\end{aligned}
$$

Table 4.7 lists typical calibration values for use with shallow wound measurements. The data are plotted in Figure 4.8.

The measured count rate in the ${ }^{239,}{ }^{240} \mathrm{Pu}$ ROI can include contributions from ${ }^{238} \mathrm{Pu}$ and ${ }^{241} \mathrm{Am}$. There is very little ${ }^{238} \mathrm{Pu}$ present in the aged plutonium mixtures at Hanford and corrections are usually not required. A method used to account for the ${ }^{241}$ Am contribution is discussed below.

\subsubsection{Adjustment to ${ }^{239} \mathrm{Pu}$ Count Rate for Presence of ${ }^{241} \mathrm{Am}$ Activity}

As discussed above, an adjustment for the ${ }^{241}$ Am activity may be required when directly measuring

${ }^{239} \mathrm{Pu} \mathrm{L} \mathrm{x}$-rays in a wound. This adjustment is made based on the ratio of count rates from the $17.5 \mathrm{keV}$ and $59.5 \mathrm{keV}$ photons (or other selected energy) from a ${ }^{241} \mathrm{Am}$ source measured at the estimated tissue depth. This ratio is applied to the net count rate in the $59.5 \mathrm{keV}$ ROI from the person's wound measurement to estimate the ${ }^{241} \mathrm{Am}$ contribution to the measured count rate in the ${ }^{239} \mathrm{Pu}$ ROI. The ${ }^{241} \mathrm{Am}$ 
contribution is then subtracted from the measured count rate in the ${ }^{239} \mathrm{Pu} \mathrm{ROI}$, as shown in Equation (4.10):

Table 4.7. Example of Calibration Data for Wound Count - No Attenuation

\begin{tabular}{ccc}
\hline Radionuclide & $\begin{array}{c}\text { Photon Energy } \\
(\mathbf{k e V})\end{array}$ & Counts/Gamma \\
\hline${ }^{241} \mathrm{Am} /{ }^{239} \mathrm{Pu}$ & 17.5 & 0.283 \\
${ }^{241} \mathrm{Am}$ & 59.5 & 0.388 \\
${ }^{235} \mathrm{U}$ & 143.8 & 0.199 \\
${ }^{235} \mathrm{U}$ & 185.7 & 0.183 \\
\hline
\end{tabular}

\subsubsection{Measurement of ${ }^{239} \mathrm{Pu}$ and ${ }^{241} \mathrm{Am}$ in Deep Wounds}

For deep ${ }^{241} \mathrm{Am}$ wounds, an estimate of the depth of the wound can be made by comparing the ratios of the $59.5 \mathrm{keV}, 21 \mathrm{keV}, 26.3 \mathrm{keV}$ and $17.5 \mathrm{keV}$ peaks to the ratios obtained from source measurements at different thicknesses of overlying tissue. A calibration factor is then determined based on the estimated depth. A similar approach is used for ${ }^{239} \mathrm{Pu}$ deep wounds using the $13 \mathrm{keV}$ and $17.1 \mathrm{keV} \mathrm{L}$ x-rays. This approach is best used when there is sufficient activity present to keep the measurement uncertainty to less than $50 \%$.

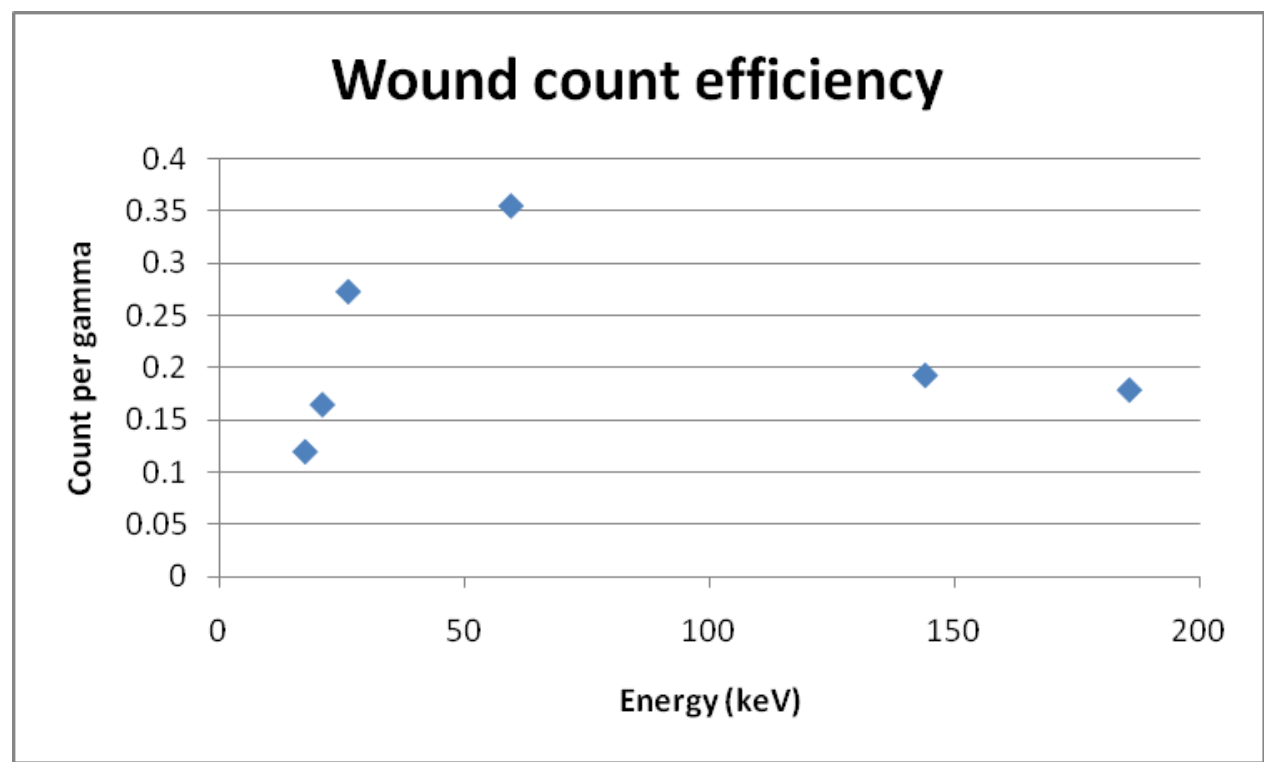

Figure 4.8. Typical Efficiency Calibration Curve for Low-Energy Wound Counting with Single Planar Ge Detector

$$
{ }^{239} \mathrm{Pu} \text { activity }=\frac{\left[\text { Person count rate in }{ }^{239} \mathrm{Pu} \text { ROI }\right]-\text { Ratio }_{17.5 / 59.5} \times[\text { Person count rate in } 59.5 \mathrm{keV} \text { ROI }]}{{ }^{239} \mathrm{Pu} \text { Calibration factor }}
$$

where ratio $17.5 / 59.5$ is the ratio of the count rate in the $17.5 \mathrm{keV}$ peak region to the count rate in the 59.5 $\mathrm{keV}$ region measured with an ${ }^{241} \mathrm{Am}$ source. 


\subsubsection{In Vivo Measurement of Fission and Activation Products in Wounds}

Injuries to the body in work areas contaminated with fission or activation products sometimes result in radioactivity being injected into the skin and underlying tissue. Preliminary measurements are made with whole body counting systems to indicate whether activity is present and at what level. Wound activity can be quantified with a large coaxial $\mathrm{Ge}$ or $\mathrm{NaI}$ detector placed in direct contact with or just slightly off the wound. The detector may be covered with a thin layer of plastic to protect it from contamination. A standard source containing the same radionuclide or a radionuclide with a similar energy is counted in the same geometry as the wound count to determine the calibration factor. Any activity in the wound is quantified by direct comparison with the activity in the standard source. The calibration factor may be obtained from a radionuclide specific calibration or from a calibration curve of efficiency versus energy. A typical efficiency versus energy calibration curve for a small wound measurement with a coaxial Ge detector is illustrated in Figure 4.9.

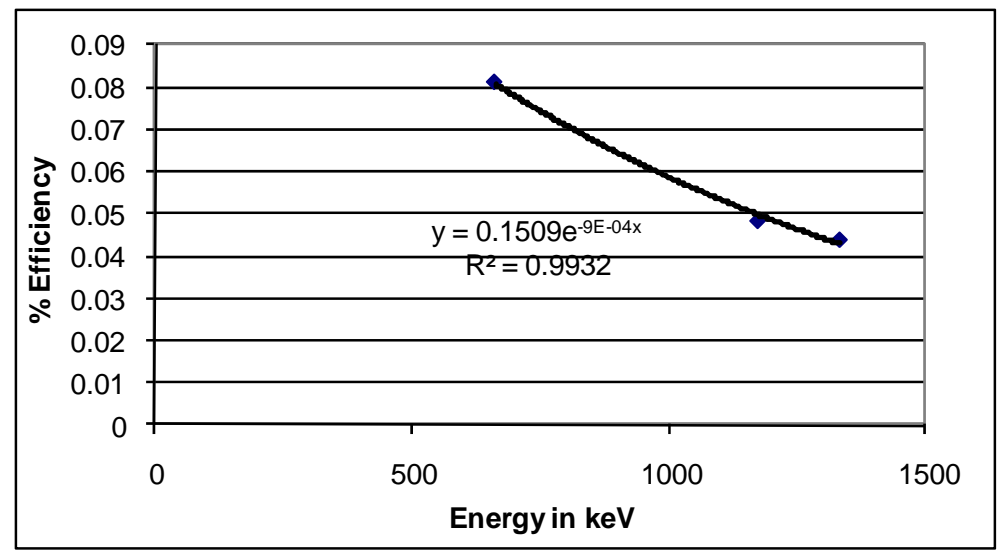

Figure 4.9. Example Wound Calibration Curve (High Energy)

For $662 \mathrm{keV} \leq$ Energy $\leq 1332 \mathrm{keV}$, a typical efficiency equation for a single coaxial Ge in contact with a small puncture wound is shown in Equation (4.11):

$$
\begin{aligned}
& \text { Efficiency }(\text { counts } / \text { gamma })=0.1509 * \exp (-0.0009 \mathrm{x}) \\
& \text { where } \mathrm{x} \text { is the energy in } \mathrm{keV} \text {. }
\end{aligned}
$$

For measurement of deep wounds, layers of tissue-substitute plastic that mimic the attenuation in soft tissue can be placed between the calibration source and the detector. These layers are used to better simulate the depth of the wound and thus provide a more accurate calibration factor for the activity determination. For large area wounds, case specific efficiency calibrations are generated.

\subsection{In Vivo Measurement of Radioactivity in the Thyroid}

For a subject with normal thyroid function, about $30 \%$ of the radioiodine entering the systemic circulation is taken up by the thyroid gland (ICRP 1989). Because of this relatively high concentration compared to other body tissues, the amount of ${ }^{131} \mathrm{I}$ and ${ }^{125} \mathrm{I}$ in the thyroid is of the most dosimetric interest. A measurement with a $38-\mathrm{cm}^{2}$ planar Ge detector is used to quantify the ${ }^{131} \mathrm{I}$ and ${ }^{125}$ I activity. The face of the detector is positioned $10 \mathrm{~cm}$ above the surface of the neck over the thyroid. The minimum 
detectable amount in the thyroid based on a 10 -minute measurement in this geometry is $140 \mathrm{pCi}$ for ${ }^{131} \mathrm{I}$ and $70 \mathrm{pCi}$ for ${ }^{125} \mathrm{I}$.

The calibration phantom is an anthropomorphic representation of the head and neck with appropriate tissue substitutes for soft tissue and bone. A hollow thyroid phantom is filled with a solid or liquid matrix that contains the appropriate calibration nuclides and is inserted into the head and neck phantom. Currently, thyroid inserts with ${ }^{133} \mathrm{Ba}$ incorporated in a tissue-substitute plastic are used for calibration to quantify ${ }^{131} \mathrm{I}$ and ${ }^{125} \mathrm{I}$ activities.

Table 4.8 provides typical calibration information for thyroid counting using a single $38-\mathrm{cm}^{2} \mathrm{Ge}$ detector positioned $10 \mathrm{~cm}$ above the surface of the neck. Typical MDA values for a 10-minute measurement are $3 \mathrm{nCi}^{125} \mathrm{I}$ and $0.5 \mathrm{nCi}^{131} \mathrm{I}$.

Table 4.8. Typical Calibration Data for ${ }^{131} \mathrm{I}$ and ${ }^{125}$ I Thyroid Measurements with Single Planar Ge Detector

\begin{tabular}{ccc}
\hline Radionuclide & Energy (keV) & Cts/s-nCi \\
\hline${ }^{125} \mathrm{I}$ & 35 & 0.020 \\
${ }^{131} \mathrm{I}$ & 364 & 0.111 \\
\hline
\end{tabular}

\subsection{In Vivo Measurement of Transuranic Nuclides in Axillary or Cubital Lymph Nodes}

Small insoluble particles of transuranic contamination that enter the lymphatic system through contaminated wounds in the extremities can be filtered out in the axillary and cubital lymph nodes, located in the area of the armpit and elbow respectively. The activity can remain in the lymph nodes for extended periods of time. Measuring this lymph node activity when the measured wound activity decreases, can help provide an indication about the mechanisms causing the activity to change at the wound site.

A single Ge planar detector is placed in light contact with the skin over the location of the lymph nodes for the measurement. Determining the location and depth of the nodes can be challenging. It may be necessary to make several mapping measurements to determine the optimum counting location. Techniques described for wound counting can be used to determine the depth of lymph nodes.

The activity in lymph nodes can be calculated manually using Equation (4.12):

$$
n C i \text { in lymph nodes }=\frac{A}{B} \cdot C
$$

$$
\text { where } \quad \begin{aligned}
\mathrm{A} & =\text { net count rate from the lymph node } \\
\mathrm{B} & =\text { net count rate from the standard source } \\
\mathrm{C} & =\mathrm{nC} \text { i in source }
\end{aligned}
$$


Knowledge of the depth of the nodes will improve the accuracy of the activity estimate although the exact depth is usually not determined. Shielding the detector from contributions from activity in other parts of the body may be necessary when large amounts of activity are present in the body.

\subsection{In Vivo Measurement of Transuranic Nuclides in Tracheobronchial Lymph Nodes}

Radioactivity in the tracheobronchial lymph nodes is difficult to detect and quantify because it is not easily distinguishable from activity in the lungs. However, if a significant amount of activity is present in these lymph nodes, it can be detected and roughly quantified by making lateral scans across the chest of a worker and comparing the count rates to those measured over a phantom. The phantom measurements would be made with lung activity only, with lymph node activity only, and with both lung and tracheobronchial lymph node activity (Northcutt et al, 1988). This measurement is usually performed using a collimated set of two Ge planar detectors. A slotted collimator that extends $5 \mathrm{~cm}$ (2 in.) below the face of the detectors has been used for this purpose.

\subsection{In Vivo Measurement of Radioactivity Using a Linear Scan}

When the fission or activation product activity is not uniformly distributed within the body, knowing the location of the activity can be useful for accurate estimates of activity and subsequent dose assessment. Rough estimates of the locations of activity can be made by a rapid measurement in the standup whole body counter. For more precise location information, a linear scan with the coaxial Ge counting system can be performed. The linear scan is not a routine counting configuration and requires considerable setup time for operation.

Calibration of the linear scanning system is performed using an anthropometric calibration phantom. This phantom allows the insertion of different sources of radioactive material into various internal organs located in the phantom. Any number of distributions and combinations of distributions can be simulated using this phantom.

The measurement is performed in the Palmer Room, using the coaxial Ge scanning system. Small magnets are placed on the bed rails at the locations of anatomical interest for the subject. These sites can include the top of head, chest, pelvis, knees, and bottom of feet. The magnets trip a microswitch that provides a signal output to the electronics, which produces a large "spike" of known magnitude in the plot of activity versus location on the body. Scanning times are set to obtain statistically valid data depending on the amount of activity in the subject.

The result of this scan can be used to determine the location of a bolus of material that a subject may have ingested for example. It may also help indicate an inhalation versus ingestion route of intake and can even track the movement and elimination of radioactive material from the gut. This measurement requires at least tens of nanocuries of fission product activity to be present to obtain a useful indication of the distribution with reasonable counting times.

\subsection{Determining CWT by Ultrasound}

When transuranic activity is confirmed to be present in the lungs, a measurement of the tissue thickness over the lungs is performed. The measurement is performed with a diagnostic ultrasound unit (Hitachi EUB-200) using a 5-MHz probe. The CWT can vary from 1.5 to $5.0 \mathrm{~cm}$ (0.6 to $2.0 \mathrm{in}$.) or more in males; the variation in females can be larger. Attenuation in the chest wall tissues can be significant. 
For ${ }^{241} \mathrm{Am}$, the half-value layer for the $59.5 \mathrm{keV}$ photon from activity distributed in the lungs is $\sim 2.2 \mathrm{~cm}$. For the $17 \mathrm{keV}$ x-rays, the half-value layer $(\mathrm{HVL})$ is $\sim 0.7 \mathrm{~cm}$. The ultrasonically measured CWT is used to help determine the appropriate calibration factor for a specific worker. It is estimated that the CWT can be measured to an accuracy of $\pm 1.5 \mathrm{~mm}$ using the ultrasound technique (Kruchten, 1990). At 17 $\mathrm{keV}$, the relative proportion of fat and muscle in the chest tissue becomes important as it relates to photon transmission. An estimate of this ratio can be obtained to improve the accuracy of the calibration factor used to estimate the activity in the lung.

\subsection{In Vivo Counting for Criticality Accidents}

The exposure of workers to a sufficiently large field of neutrons will result in induced radioactivity in the body from the activation of elements such as calcium, nitrogen, sodium, and chlorine.

A neutron dose of $20 \mathrm{mrad}$ of fission spectra neutrons will produce approximately $10 \mathrm{nCi}$ of ${ }^{24} \mathrm{Na}$ in the body. Immediately after exposure ${ }^{49} \mathrm{Ca},{ }^{24} \mathrm{Na},{ }^{38} \mathrm{Cl},{ }^{28} \mathrm{Al}$, and ${ }^{13} \mathrm{~N}$ are easily measured, but because of the short half-lives, only ${ }^{24} \mathrm{Na}$ would normally be measurable by the time the worker is transported from the work site to the IVRRF. The assessment of ${ }^{24} \mathrm{Na}$ in workers exposed to neutrons from a criticality accident provides a means of estimating the neutron dose.

After the gamma-ray emitters from the induced activity have decayed, measurements of bremsstrahlung radiation from ${ }^{32} \mathrm{P}$ in the bone may give information on which portions of the body received the highest dose.

\subsubsection{Measuring Activity Resulting from Criticality Accidents}

Since the activity of ${ }^{24} \mathrm{Na}$ in the body of the worker exposed to a criticality event may be relatively high, the procedure used to evaluate the amount will depend on the quantity present.

Low levels can be quickly assessed with a measurement using the standup whole body counter. For a more precise assessment or for levels that exceed the standup counter's capability, measurements can be made with the coaxial Ge system. An alternative method using a single NaI detector and a tilted chair or meter arc geometry could also be employed although this would require significant setup time.

\subsubsection{Background Correction}

To obtain the net count rate, the background rate is subtracted from the gross rate. The background may be calculated from the worker's spectrum, a previously determined background, or from an appropriate fraction of a long background count done with an uncontaminated person. A background count with the detector in the same position as used for the worker's measurement should be used for the precise calculation of the background. By the time the exposed person arrives at the IVRRF, most interfering radionuclides will usually have decayed to insignificant levels.

\subsubsection{Calculations of Activity}

The estimate of ${ }^{24} \mathrm{Na}$ activity in the body can be used to determine the average fast neutron dose. The routine algorithms for the standup and coaxial Ge systems are the primary method to obtain estimates of the ${ }^{24} \mathrm{Na}$ activity using the $1369 \mathrm{keV}$ photon (100\% abundance) or the $2754 \mathrm{keV}$ photon at $99.9 \%$ abundance. Results from these systems may be subject to interferences and dead time and would be closely scrutinized. 


\subsubsection{Calculating the ${ }^{24} \mathrm{Na}$ Activity (Historical Method)}

Alternatively, the calibration factor for determining the body burden of ${ }^{24} \mathrm{Na}$ using a $23.8 \mathrm{~cm} \times 10 \mathrm{~cm}$ (9.375 in. $\mathrm{x} 4 \mathrm{in}$.) diameter $\mathrm{NaI}(\mathrm{Tl})$ detector in the tilted chair position is $6522 \mathrm{cpm} / \mu \mathrm{Ci}$. The counts per minute are determined from the $2.75-\mathrm{MeV}$ peak using channels 128 to 143 inclusive. Equation (4.13) determines the total body content of ${ }^{24} \mathrm{Na}$ :

$$
\mu \mathrm{Ci} \text { of }{ }^{24} \mathrm{Na} \text { in the body }=\frac{\text { net counts } / \mathrm{min}}{6522}
$$

The calibration factor based on the average of measurements on two individuals is $2.94 \mathrm{E}-03$ counts/gamma. Further documentation can be found on pages 131-137 in the Laboratory Notebook \#1 HWN-2454. (Note: This method requires significant setup time.)

\subsubsection{Calculating the Neutron Dose}

The average total-body fast neutron dose calculated from total body ${ }^{24} \mathrm{Na}$ measurements is determined using Equation (4.14) as follows (Hanford Laboratories 1963; Harris 1961):

$$
\text { dose in rads }=\frac{{ }^{24} \mathrm{Na} \text { activity in } \mu \mathrm{Ci}}{\text { weight of subject in } \mathrm{kg}} \cdot 226
$$

\subsection{Cited References}

Hanford Laboratories, General Electric Company. 1963. "Dosimetry Investigation of the Recuplex Criticality Accident." Health Physics 9(7):757-768.

Harris, P. S. 1961. "Special Supplement on Acute Radiation Death Resulting from an Accidental Nuclear Critical Excursion." Journal of Occupational Medicine 3(3):179-180 (available in the IVMPH Library).

ICRP Publication 56. Age Dependent Doses to Members of the Public from Intake of Radionuclides:Part 1, Volume 20, No. 2. 1989. International Commission on Radiological Protection. Pergamon Press. London.

Kruchten, D.A., Anderson, A.L., "Improved Ultrasonic Measurement Techniques Applied to Assay of Pu and Other Transuranics in Lung", Health Physics Journal, Vol. 59 Issue 1, July 1990.

Lynch, T.P., Kathren, R.L., McInroy, J.F. 1988. "Macordistribution of Plutonium and Americium in Four Human Skeletons", J. Soc. Radiat. Protect. 8:67-76.

Lynch, T.P., Kathren, R.L., Dagle, G.E., McInroy, J.F. 1989. "Comparative Skeletal Distribution of Am and Pu in Man, Monkey, and Baboon". Health Physics 57 Supplement 1, 81-88.

Neton, J. W. 1988. "A Method for the In Vivo Measurement of Americium-241 at Long Times PostExposure.” Ph.D. dissertation, New York University.

Northcutt, A. R., S. E. Binney, and H. E. Palmer. 1988. "In-Vivo Counting of ${ }^{241} \mathrm{Am}$ in Human Lungs and Tracheo-bronchial Lymph Nodes." Health Physics 54(1):73-81. 
Palmer, H. E. and G. A. Rieksts. 1989. "Improving Counting Efficiencies for Measuring ${ }^{239} \mathrm{Pu}$ in the Lung in the Sitting Position.” Health Physics 57(5):747-752.

Palmer, H. E., G. A. Rieksts, and E. E. Icayan. 1983. “1976 Hanford Americium Exposure Incident: In Vivo Measurements.” Health Physics 45(4): 893-910.

Palmer, H. E. and W. C. Roesch. 1965. "A Shadow-Shield Whole Body Counter." Health Physics 11:1213-1219.

Pacific Northwest National Laboratory (PNNL). PNL-MA-554, R\&HT In Vivo Monitoring Program Procedures Manual. Richland, Washington. (Internal manual)

\subsection{Other Useful References}

ICRP Publication 38. Radionuclide Transformations, Volume 11-13. 1983. International Commission on Radiological Protection. Energy and Intensity of Emissions. Pergamon Press. London.

Kocher, D. C. 1981. Radioactive Decay Data Tables. DOE/TIC-11026, National Technical Information Service, Springfield, Virginia.

Palmer, H. E., H. B. Spitz, and G. A. Rieksts. 1985. "Part III: Gamma-Ray Measurements.” Health Physics 49(4):577-586.

Palmer, H. E. and M. C. Rhoads. 1989. "Determining the Ratio of ${ }^{239} \mathrm{Pu}$ to ${ }^{241} \mathrm{Am}$ in Wounds by Measuring the L $\gamma$ X-Rays with a Si(Li) Detector." Health Physics 56(2):249-252. 
IN VIVO MONITORING PROGRAM MANUAL

PNL-MA-574

\author{
SECTION 5.0 \\ QUALITY ASSURANCE
}




\section{Contents}

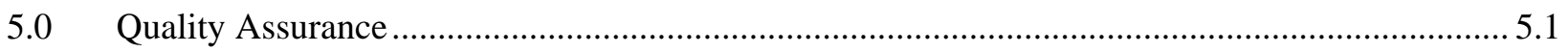

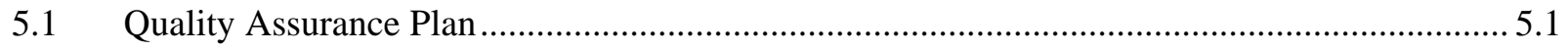

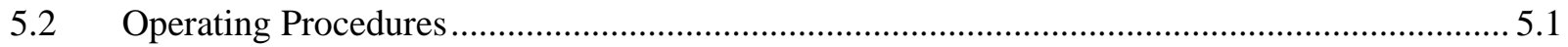

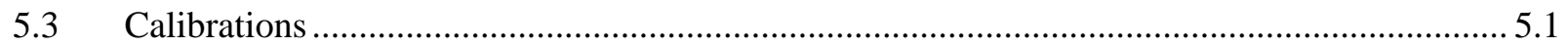

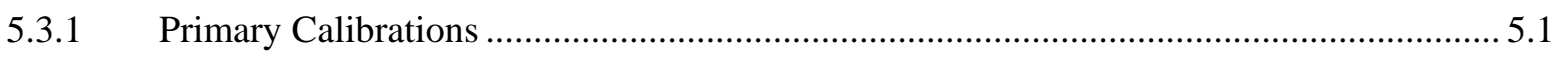

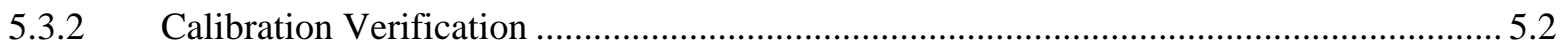

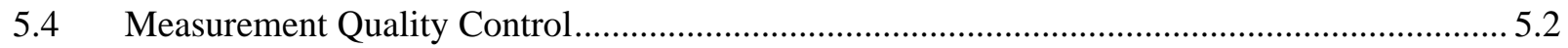

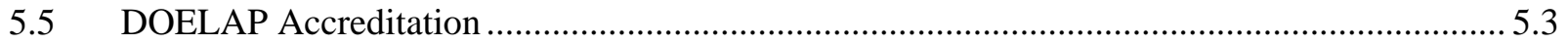

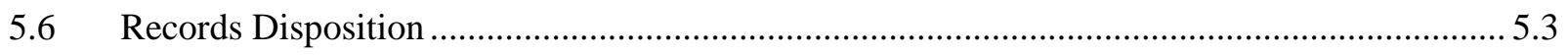

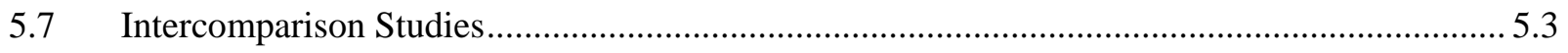

5.8 Computer Code Verification, Validation, and Documentation................................................ 5.3

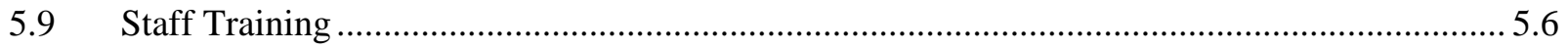

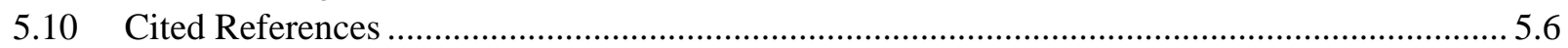




\subsection{Quality Assurance}

The quality assurance (QA) program for the In Vivo Monitoring Program (IVMP) includes the structure and controls to guide proper conduct of operations and the generation of quality results.

\subsection{Quality Assurance Plan}

The Radiation \& Health Technology (R\&HT) Quality Assurance Program Plan (QAPP) was issued in June 2004 to address the quality program common to all the R\&HT programs. The QAPP supplements the Pacific Northwest National Laboratory (PNNL) Quality Assurance Program as detailed in the Quality subject area of PNNL's How Do I (HDI) system and implements the requirements from 10CFR830, Subpart A, DOE Order 414.1C, and NQA-1-2000 through a graded approach. The QAPP addresses the program's organization, quality assurance, design and software control, documents, procurement, measuring and test equipment, handling, storage and shipping, nonconformances and corrective actions, records management, assessments and quality improvements, service to clients, complaints, and communicating and reporting to clients. Appendix C of the R\&HT QAPP contains a matrix that crossreferences the Department of Energy Laboratory Accreditation Program (DOELAP) requirements to the corresponding IVMP documentation.

\subsection{Operating Procedures}

The In Vivo Monitoring Program Procedures Manual, PNL-MA-554, contains the detailed operating procedures that are used to control the routine operations. The procedures are controlled to ensure that the most recent version of a procedure is being used, that the procedure received the proper review and approval before implementation, and that adequate change control is implemented. Staff training to procedures is accomplished and documented for each staff member in accordance with procedure AP-12, R\&HT Training Program, within the PNL-MA-870 manual. The procedures are reviewed at least every 2 years.

\subsection{Calibrations}

The proper functioning and stability of the in vivo counting systems are maintained and monitored using a combination of primary calibrations, calibration verification measurements, quality control (QC) measurements, and data review and analysis.

\subsubsection{Primary Calibrations}

The in vivo counting systems are periodically calibrated using anthropometric phantoms that simulate the size, shape, and composition of the human body. The results from the measurements of the calibration phantoms are used to calculate the calibration factors that are used to convert the measured count rate from a worker to an estimate of the activity measured in the body. The radioactive sources (except ${ }^{40} \mathrm{~K}$ ) used in the phantoms are traceable to the National Institute of Standards and Technology (NIST) or are cross calibrated to sources with a NIST pedigree. The calibrations are carried out in accordance with established procedures.

At the time phantom measurements are made, measurements are also made of check sources that are used to characterize the performance of the system. Control charts are generated based on the systems' response to the check sources and are used to monitor the systems' counting efficiency, resolution, and 
gain stability. If the system performance is within the control chart limits, this demonstrates that the systems performance is comparable to the performance at the time when the calibration measurements were performed. This provides objective evidence that the calibration factors are applicable to the workers' measurements.

Primary calibrations of the in vivo counting systems are performed at 3-year intervals as a minimum. Primary calibrations may also be performed whenever the daily source count results cannot be reproduced within the control chart limits established at the time of the last calibration or when major changes (e.g., detector change) are made to counting systems that can affect the system performance.

Energy calibrations and resolution calibrations are also established to provide the energy per channel relationship for each spectrum and the system resolution as a function of energy that is used for peak identification and to establish the size of the energy regions of interest (ROI) used in the calculations.

\subsubsection{Calibration Verification}

During the time between the primary calibrations, several measurements are made using the calibration phantoms. The results from these measurements are used to verify the calibration factors derived from the primary calibrations on an ongoing basis. Measurements are made of selected phantoms during the year. The results from the phantom measurements are used in conjunction with the daily quality control measurements to evaluate the performance of the in vivo counting systems.

After maintenance, repair, upgrade or other changes that can affect the calibration parameters, the performance of the system is checked before in vivo measurements are performed. In addition to the quality control checks, measurements of selected calibration phantoms may be made. If the response of the system differs significantly from the previous primary calibration values, a complete recalibration of a system may be warranted. The decision whether a recalibration is required is made by the Program Manager (PM).

\subsection{Measurement Quality Control}

Daily efficiency checks, centroid checks, energy calibration checks, and background measurements are made on each of the routine counting systems. The efficiency check is performed in the morning before counting workers and again in the afternoon after the last worker is counted. As noted previously, the control chart limits are established for these parameters at the time when a primary calibration is performed. Results outside the specified control chart limits are investigated and corrective actions are taken as appropriate. The results from the daily quality control checks are trended and evaluated quarterly.

The results from the daily background measurements are used to determine if there has been an unexpected change in the background count rate that would preclude the use of a system for routine counting. The background measurement results are also trended quarterly to evaluate whether subtle changes have occurred that might indicate degradation in the performance of the measurement system or contamination of the counting room.

The measurement QC program is designed to maintain the performance of the measurement systems within $\pm 10 \%$ based on the response to the check sources. The number of QC measurements routinely performed represents at least $5 \%$ of the total number of measurements performed annually. This meets the DOELAP requirement that the minimum number of QC measurements performed should be $5 \%$ of the total number of measurements. 
The results from the QC measurements are retained as program records.

\subsection{DOELAP Accreditation}

The IVMP is accredited through the DOELAP for direct radiobioassay. The program requires the participating laboratories to maintain the accreditation on a three-year cycle. A copy of the DOELAP certificate is shown in Figure 5.1 and the conditions of accreditation are shown in Figure 5.2. DOELAP accreditation has been continuously maintained since it was initially conferred in February 1998.

\subsection{Records Disposition}

Program records are temporarily stored at the In Vivo Radiobioassay and Research Facility (IVRRF) and are transferred to the Hanford Radiological Records Program (HRRP) and to PNNL Records Management for archival and permanent storage. Working copies of the records may be retained at the IVRRF. Electronic files containing the results from the in vivo measurements are periodically transferred to the Hanford Radiological Exposure System (REX) database.

The IVMP File Plan (formerly Records Inventory and Disposition Schedule) is maintained. The File Plan documents the inventory of program records and their disposition. Section 7.0 contains a more detailed discussion on the program records.

\subsection{Intercomparison Studies}

The IVMP staff may participate in intercomparison studies as another tool to evaluate the reliability of the in vivo measurement data. At a minimum, the IVMP staff participates in DOELAP performance testing every three years.

\subsection{Computer Code Verification, Validation, and Documentation}

Computer codes used as part of the IVMP that directly affect the final data are managed under a configuration management plan contained in procedure IVMP300.B.10. The purpose of the plan is to ensure proper verification, validation, change control, and documentation of the codes. 

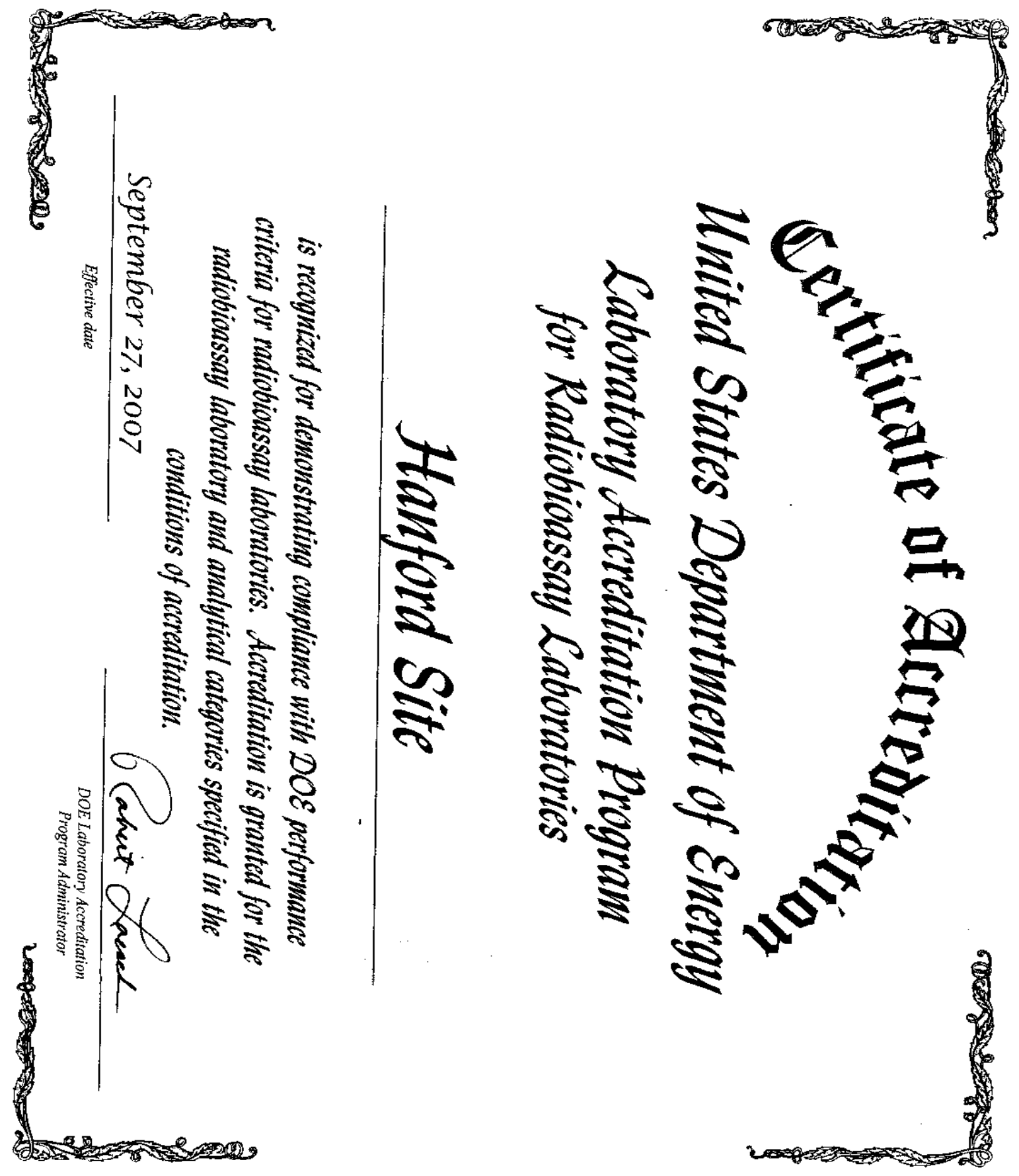

Figure 5.1. DOELAP Certificate 


\section{CONDITIONS OF DOELAP ACCREDITATION}

\section{Hanford Site}

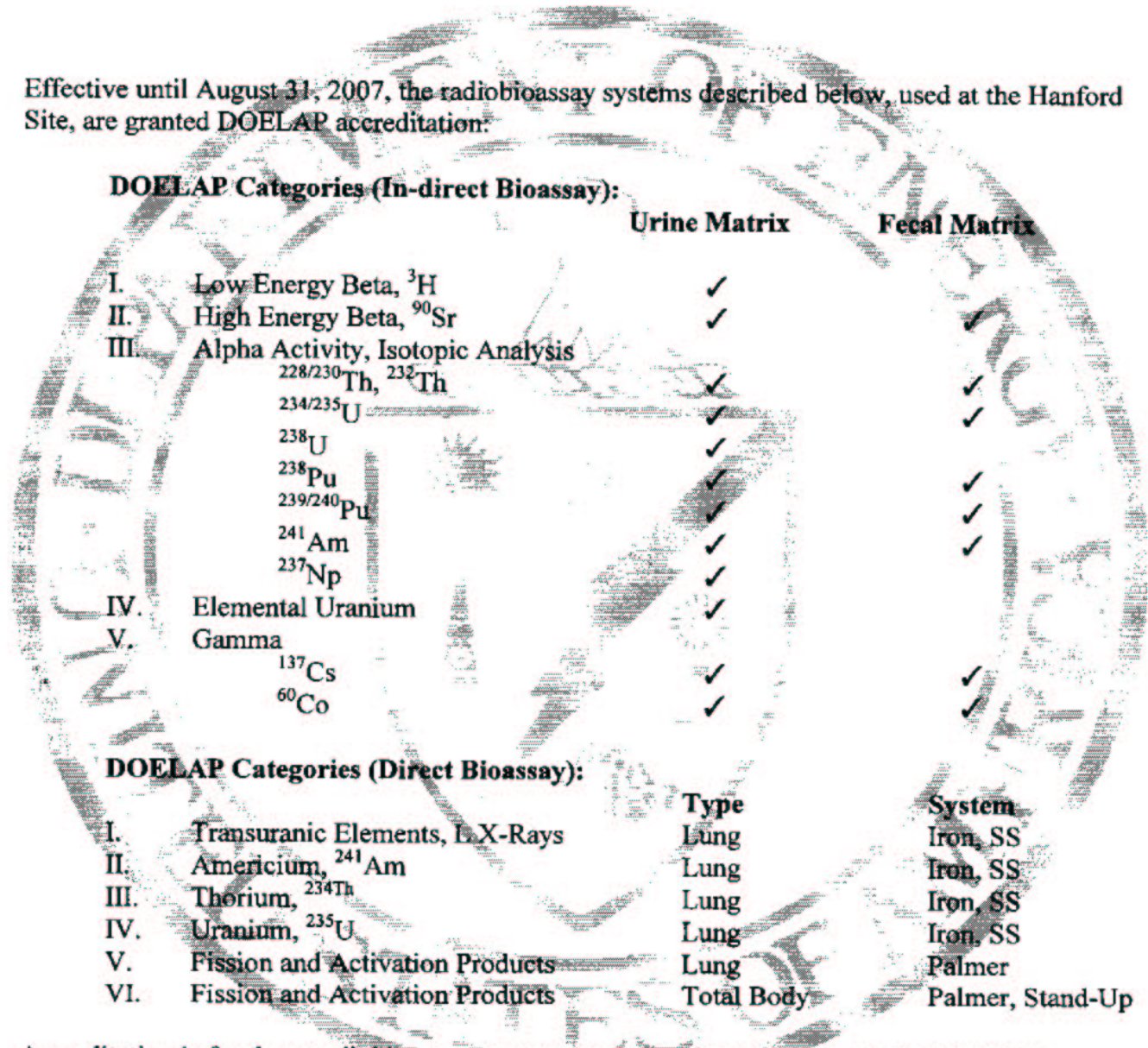

Accreditation is for these radiobioassay programs only and is contingent upon maintaining radiobioassay practices that are consistent with the methodologies used during DOELAP performance testing and the onsite assessment.

Figure 5.2. Conditions of DOELAP Accreditation 


\subsection{Staff Training}

The staff are qualified and properly trained for all the work performed under the IVMP in accordance with PNL-MA-870 manual, procedure AP-12, R\&HT Training Program. The PM assigns the required training. A combination of laboratory-wide training, procedure use training, and job specific training is used to keep the staff current on pertinent procedural, technical and safety-related topics. The staff performance is reviewed annually. Staff competency is evaluated on a routine basis. All staff training is documented.

\subsection{Cited References}

American National Standards Institute (ANSI). 1996. Performance Criteria for Radiobioassay. ANSI Standard N13.30, New York.

DOE Order 414.1C, Quality Assurance, U.S. Department of Energy (DOE), June 17, 2005, Washington, D.C.

Pacific Northwest National Laboratory (PNNL). R\&HT, Quality Assurance Program Plan. Richland, Washington. (Internal manual)

Pacific Northwest National Laboratory (PNNL). PNL-MA-554. R\&HT In Vivo Monitoring Program Procedures Manual. Richland, Washington. (Internal manual)

Pacific Northwest National Laboratory (PNNL). PNL-MA-870. R\&HT Training Program, AP-12, R\&HT Administrative Processes. Richland, Washington. (Internal manual)

How Do I (HDI) system, Pacific Northwest National Laboratory.

U.S. Department of Energy. 1998. DOE Laboratory Accreditation Program for Radiobioassay. DOESTD-1112-98. Washington. D.C.

10CFR830, Subpart A, Quality Assurance Requirements, U.S. Department of Energy (DOE).

Washington, D.C. 


\title{
IN VIVO MONITORING PROGRAM MANUAL
}

\author{
PNL-MA-574
}

\author{
SECTION 6.0
}

IN VIVO MEASUREMENT DATA PROCESSING AND ANALYSIS 


\section{Contents}

6.0 In Vivo Measurement Data Processing and Analysis ............................................................. 6.1

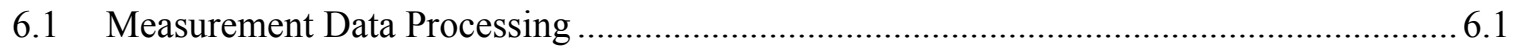

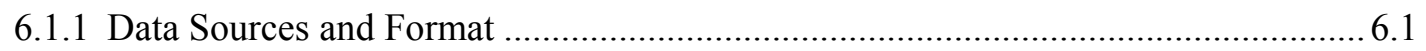

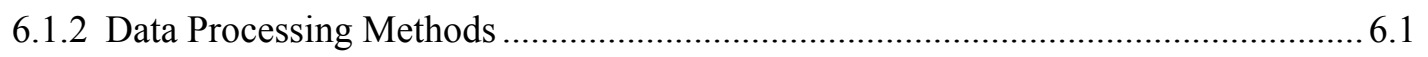

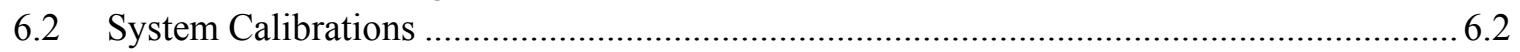

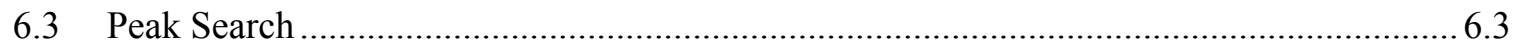

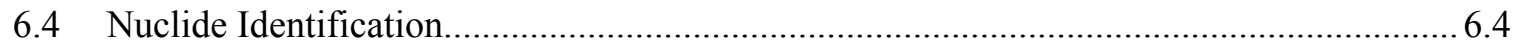

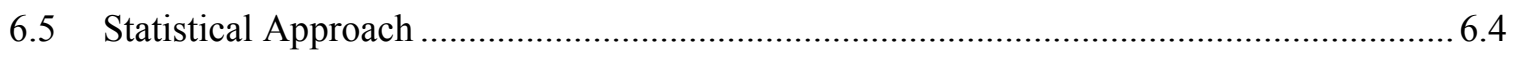

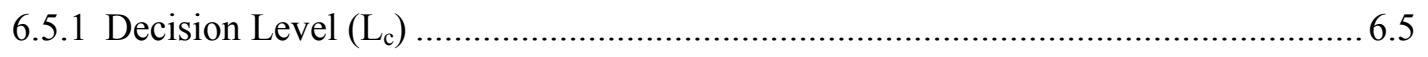

6.5.2 $\mathrm{L}_{\mathrm{c}}$ Determination for Multiple Nuclide Analysis ................................................ 6.5

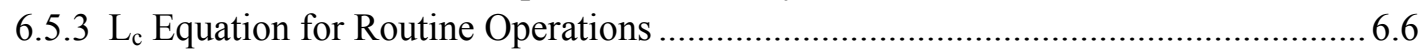

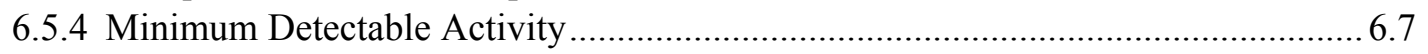

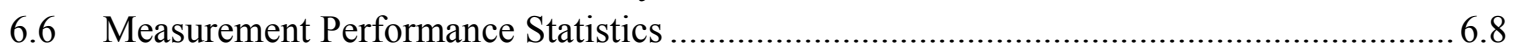

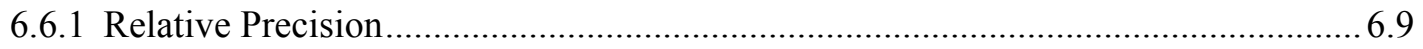

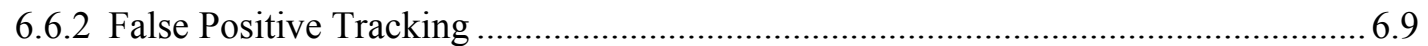

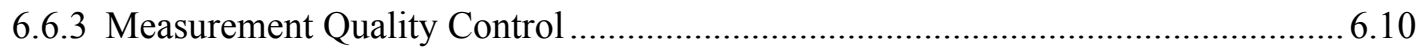

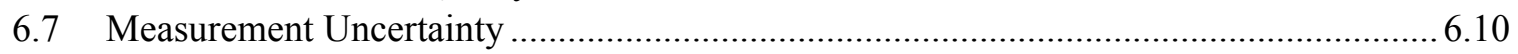

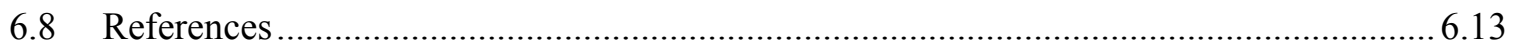




\subsection{In Vivo Measurement Data Processing and Analysis}

\subsection{Measurement Data Processing}

The routine data processing functions and most other data processing are computerized. The following are general functions provided by the application software:

- acquiring spectra from individual and multiple detectors,

- $\quad$ summing spectra from different detectors into a composite spectrum for analysis,

- incorporating descriptive alphanumeric information with the spectra into an easily manageable record,

- $\quad$ analyzing the spectrum, calculating results, and storing results in a database record,

- retrieving records for recalculation, plotting, printing, and/or tabulating results for analysis of grouped data,

- creates data files that are uploaded to the Radiological Exposure System (REX) that is maintained by the Hanford Radiation Records Program,

- providing count information for cost accounting and other statistical reports.

\subsubsection{Data Sources and Format}

Each measurement represents a spectrum or histogram of the number of energy deposition events as a function of the radiation energy detected by a given detector system in a specific counting geometry. The spectral information, results, and the demographic data are stored as electronic configurations in binary format. The results summary and demographic data are extracted from the configuration, and placed in a text file that is transferred to REX. Paper copies of the measurement results are generated, reviewed, and ultimately sent to records storage.

\subsubsection{Data Processing Methods}

Since October 1999, the spectral data from the measurement systems have been acquired, reduced, manipulated, and analyzed using the Abacos Plus-Based Counting System (ABCS) software. Dozens of command procedures were written to customize the software specifically for Hanford measurements. The analysis parameters used are either stored electronically or as part of the calibration documentation for the specific measurement system. Non-routine calculations are performed by professional staff and are peer reviewed prior to being entered as an official record.

The ABCS software resides on a Hewlett Packard Model DS-10 workstation running the Open VMS operating system. The workstation is connected to the acquisition interface modules or digital processing modules and remote operator terminals via a local area network. Terminal emulation software is used for 
remote access to the workstation via the Pacific Northwest National Laboratory (PNNL) network. The workstation is also connected to the PNNL network to allow communication with REX.

All spectra are assigned a unique tagword identification (e.g. 10A0001 is the first count performed in 2010) and combined with descriptive alphanumeric information, such as name, payroll number, social security number, etc. The combination of a spectrum, alphanumeric descriptors, count specific parameters, peak search results, and the calculated results is stored in the computer's database. This database is accessed to plot, print, calculate, or do statistical analyses on the spectral records.

The results are calculated for specific radionuclides of dosimetric importance at Hanford. Quantitative results are saved for the radionuclides listed in the nuclide library whether the result exceeds the decision level, is zero, or is less than zero.

\subsection{System Calibrations}

At the most basic level, the analysis of in vivo spectral data involves the conversion of net count rates obtained from worker measurements in specific energy regions of interest (ROI) to an estimate of the radionuclide activity. This section provides an overview of the analysis methods used to convert the net count rate to an activity estimate.

The ABCS is used to establish the energy, resolution, and efficiency relationships as a function of photon energy for whole body counts. For lung counting applications, the efficiency is expressed as a function of both energy and chest thickness. Additional detail is found in the Genie-VMS Abacos Plus User's Manual. The software applies these parameters to the acquired spectrum to quantify the amount of a radionuclide in the body. Results are calculated for each of the radionuclides contained in the analysis library. The energy calibrations are used to set the energy per channel values that define a system's dynamic energy range and allows identification of the peak energies. The resolution calibration defines the system resolution as a function of photon energy and is used to set the size of the ROI that is used to determine the net counts and ultimately the activity value. The efficiency calibrations establish the factors used to convert count rates to activity estimates. For whole body counting the efficiency calibration is typically expressed as a function of energy. The coaxial germanium scanning system utilizes two efficiency calibrations. One is for workers weighing less than $200 \mathrm{lbs}$. and another for workers weighing $200 \mathrm{lbs}$. or more. For lung counting, the efficiency for a specific energy is typically expressed as an exponential function of the chest thickness. The software provides the option to describe the efficiency versus energy relationship using either an empirical function or a cubic spline function. The empirical function fits the data using a polynomial with a degree between 2 and 5 and is used primarily for whole body counting calibrations. The cubic spline function constrains the series of $3^{\text {rd }}$ degree polynomials so that the curve passes through each calibration point and is used primarily for chest and organ counting. The empirical efficiency function has the general form:

$$
\begin{aligned}
& \operatorname{Ln}(\text { Efficiency })=a_{2}+a_{3} x+a_{4} x^{2}+a_{5} x^{3}+a_{6} x^{4}+a_{7} x^{5} \\
& \text { where } \quad \begin{array}{l}
x=\ln \left(a_{1} / E\right) \\
E=\text { peak energy in keV } \\
a_{1}=(E 2+E 1) / 2 \\
E 2=\text { largest calibration energy } \\
\text { E1 }=\text { smallest calibration energy }
\end{array}
\end{aligned}
$$

By default, the number of fit parameters $\left(a_{2}-a_{7}\right)$ and the polynomial degree is determined by the Abacos empirical fitting algorithm. The number of parameters depends on the number of calibration 
energies used, the overall shape of the curve and the uncertainty in the efficiency values. The algorithm uses the reduced chi-square statistic as a goodness-of-fit indicator. A value close to one (e.g. 0.8 to 1.2) is desirable. If the chi-square statistic exceeds 1.2, the software repeats the fit adding one more term in the polynomial. The iterative process continues until the reduced chi-square value is less than 1.2 or until a 5 th degree polynomial is reached. Conversely, if the reduced chi-square value is less than 0.8 , indicating an over determined data set, the fit is repeated by reducing the degree for the polynomial. The process is repeated until the reduced chi-square value exceeds 0.8 or until a second degree polynomial is reached. The user has the option to override the software to select the polynomial degree.

For chest counting calibrations, the efficiency at a specific energy is calculated as a function of the chest wall thickness (CWT) as shown in Equation 6.2:

$$
\text { Efficiency }=\mathrm{b}_{1} \mathrm{e}_{2}{ }_{2}^{*} \mathrm{CWT}
$$

The constants $b_{1}$ and $b_{2}$ are specific to the fitted curve.

\subsection{Peak Search}

A detailed description of the peak search algorithm is found in the Peak Search Program Algorithm (07-0064) document. An overview of the information in the document is provided below.

Peak location is accomplished through the use of a correlation function, which is used to calculate a correlation value for each channel in the spectrum. A correlation value in the discrete case of a multichannel analyzer (MCA) spectrum is very similar to a second derivative in the continuous case. Thus, if the program is sampling a portion of the spectrum that contains no peaks, the correlation value should be very close to zero. Conversely, a very large negative second derivative value will occur near the centroid channel of a peak. When the program finds a portion of the spectrum that shows peak-like characteristics, it then rescans the region to see if the region comprises more than one peak.

Many criteria must be satisfied before a peak is considered to be identified by the peak search program. The user sets numerous parameters for the peak search analysis including the start and end channels, peak search sensitivity, and the Gaussian shape sensitivity. The peak search sensitivity parameter represents the minimum number of standard deviations above the background level that a background-subtracted peak must be in order to be identified as a peak. The Gaussian shape sensitivity parameter determines how close to a pure Gaussian shape identified peaks need to be. If a peak deviates from the Gaussian shape by a percentage greater than the Gaussian sensitivity value, it is flagged as a possible multiplet. Suspected multiplets are rescanned using a correlator function with a smaller width. The correlator value is checked at each point. If it is greater than a value that depends linearly on the Gaussian sensitivity parameter, the channel will be recognized as a peak.

Once a peak is located, the program quantifies the peak area by calculating the start and end channels, the exact centroid channel, full width at half maximum (FWHM), gross count rate, background count rate, and the net count rate. If the region includes multiplets, the program performs a least squares fit to determine the individual values. When all peaks are found and quantified a report is automatically generated.

If background peaks (e.g. ${ }^{234} \mathrm{Th},{ }^{226} \mathrm{Ra},{ }^{228} \mathrm{Ac}$ ) are present in a room spectrum, an environmental background correction can automatically be performed. The environmental background spectrum is obtained for a counting system with an anthropometric phantom present that contains no added radioactive material. The phantom is used for this measurement to approximate the shielding, 
attenuation, and scatter from a worker. The Environmental Background (ENBACK) program searches for common peaks found in both the background spectrum and the worker's spectrum. For the common peaks, ENBACK subtracts the area of the background peak from the total area of the peak in the person's spectrum taking into account the differences in counting times. Common peaks are defined as peaks that are within the nuclide identification (NID) process energy tolerance. Another option allows the subtraction of an environmental peak when no corresponding peak is identified in a subject's spectrum.

\subsection{Nuclide Identification}

After peaks in the spectrum are identified, the analysis process proceeds to associate the peaks with the radionuclides that generated the photons as a result of their radioactive decay. This NID also calculates the activity of each nuclide. A more detailed description of the nuclide identification algorithms is given in the Spectroscopy Algorithms and Software Verification and Validation Manual (070368-03).

Before any of the nuclide identification commands can be used a nuclide library file must be created. The file contains fields for nuclide-specific information including nuclide symbol, half-life, half-life uncertainty, nuclide type (e.g., fission, natural, etc.), gamma-ray energies, percent abundance, and abundance uncertainty.

The NID scans the peak search results for an energy line that matches the energy in the nuclide library within the user-selectable energy tolerance. The energy tolerance test, half-life test, peak origin test, and abundance test are user selectable parameters that can be used as part of the identification process. More detailed information on this and NID in general is contained in the Genie VMS Spectroscopy System Users Manual, Model 48-0198.

In the vast majority of the in vivo measurements, peaks associated with the nuclides of dosimetric importance at Hanford are not identified. In these cases, the ABCS employs a "forced activity calculation" to quantify the activity. For each energy line listed in the library, the energy is converted to a centroid channel based on the energy calibration. The product of a user selected variable peak width value multiplied by the resolution value is used to define the number of channels in the peak region. The software adjusts the size of the region if there are interfering peaks. A linear estimate of the continuum background is calculated, net counts are determined by subtracting the background estimate from the gross counts in the peak ROI, and finally the calibration factor is applied to calculate the activity. In addition, a time normalized environmental background is subtracted for peaks that are identified in the room background spectrum as discussed above. The method used to estimate the background is described in the Canberra Spectroscopy Applications Algorithms and Software Verification and Validation Manual (07-0368-03) and the VMS Software Service Newsletter, March 1994.

\subsection{Statistical Approach}

This section describes the statistical approach used to calculate radionuclide activity estimates from in vivo measurements, based on the guidance found in ANSI N13.30, Performance Criteria for Radiobioassay (ANSI 1996); DOE-STD-1112-98 (1998), and other national and international standards.

The use of the decision level $\left(\mathrm{L}_{\mathrm{c}}\right)$ and the minimum detectable activity (MDA) as they pertain to the interpretation of direct in vivo measurements are described here. Additional statistical analyses including mean blank value (MBV) and the use of control charts are also discussed. 


\subsubsection{Decision Level $\left(L_{c}\right)$}

For routine in vivo measurements, activity is considered to be detected when a measurement result is above the $\mathrm{L}_{\mathrm{c}}$ value. As defined in ANSI N13.30, the $\mathrm{L}_{\mathrm{c}}$ value is the amount of a count or final instrument measurement of a quantity of analyte at or above which a decision is made that a positive quantity of the analyte is present. For analysis of a single nuclide with no interfering signals, and an acceptable false positive probability defined as $5 \%$, the calculated $\mathrm{L}_{\mathrm{c}}$ value using Equation 1 of ANSI N13.30 is $2.33 \mathrm{~s}_{\mathrm{b}}$ plus a set fraction of the baseline count, where $\mathrm{s}_{\mathrm{b}}$ is the estimated standard deviation of the gross measurement made on a blank (ANSI 1996; Currie 1984).

Each of the thousands of measurements performed at the IVRRF each year is used to simultaneously estimate the activity for multiple radionuclides. The activity calculation for each photon energy is treated independently to ensure that any detectable radionuclides are measured accurately. In setting the $\mathrm{L}_{\mathrm{c}}$ based on a $5 \%$ false positive rate for each of three nuclides in the library, $14 \%$ of all spectra collected that contain no activity from the radionuclides in the library would theoretically be expected to show at least one false positive photopeak. This would be an unacceptably high recount rate for routine operations. Consequently, the program is designed for an expected false positive rate of $\leq 5 \%$ per measurement (i.e., one or more results above the Lc value) as described in Section 6.5.2.

The $\mathrm{L}_{\mathrm{c}}$ value is calculated according to Equation 6.5 for all radionuclides at Hanford that are of dosimetric interest. These radionuclides are included in the analysis libraries and quantitative estimates of their activity are routinely calculated. In addition, a peak search algorithm as described in Section 6.3 is used to scan the entire spectrum to identify peaks associated with other nuclides that could be present but are not expected to be detected.

The large number of in vivo measurements performed annually at the IVRRF can result in a significant number of recounts due to false positive results. Also, a percentage of the measurements on subjects with an MDA amount of radioactive material in their body will be falsely negative. The IVRRF optimizes the probabilities of both false positive and false negative results through judicious setting of the error rates, while maintaining acceptable MDA values. Section 6.5.4 addresses the formulation of the MDA.

\subsubsection{Lc Determination for Multiple Nuclide Analysis}

The number of results greater than the $\mathrm{L}_{\mathrm{c}}$ value observed when no radioactive material is present (i.e., false positives) will follow a binomial distribution. The probability of observing a result less than the $\mathrm{L}_{\mathrm{c}}$ is, therefore, equal to 1 minus the probability of observing a result greater than the $L_{c}(1-\alpha)$. The probability of observing a result less than the $\mathrm{L}_{c}$ " $\mathrm{n}$ " times in a row, given that there is no activity present, then equals $(1-\alpha)^{\mathrm{n}}$. If the overall false positive probability for the measurement $(\mathrm{P})$ is set at $5 \%$, the probability of observing a count less than the $\mathrm{L}_{\mathrm{c}}$ in all of the " $\mathrm{n}$ " ROI is $95 \%$ and the probability $(\alpha)$ of observing a count greater than the $\mathrm{L}_{\mathrm{c}}$ in any of the ROI is calculated as follows:

$$
\begin{aligned}
(1-\alpha)^{n} & =(1-P) \\
(1-\alpha)^{n} & =(1-0.05) \\
(1-\alpha)^{n} & =(0.95) \\
(1-\alpha) & =\sqrt[n]{0.95} \\
\alpha & =1-\sqrt[n]{0.95}
\end{aligned}
$$


The false positive probability ( $\alpha$ ) for calculations using up to three regions of interest (ROI) (i.e., $n$ equals 3 ) is $1.7 \%$ per nuclide. Table 6.1 presents the calculated probabilities of observing a false positive result when analyzing for one or more radionuclides. $\mathrm{K}_{\alpha}$ is the abscissa of the standardized normal distribution corresponding to the probability level of 1- $\alpha$ (ANSI 1996).

Table 6.1. Calculated Probability of False Positive Results and Associated $\mathrm{K}_{\alpha}$ for Total 5\% Probability of One or More False Positive Results per "n” ROI

\begin{tabular}{rcc}
\hline $\mathbf{n}$ & $\boldsymbol{\alpha}$ & $\mathbf{K}_{\boldsymbol{\alpha}}$ \\
\hline 1 & 0.05000 & 1.645 \\
3 & 0.01695 & 2.122 \\
4 & 0.01274 & 2.235 \\
5 & 0.01021 & 2.319 \\
8 & 0.00639 & 2.490 \\
10 & 0.00512 & 2.569 \\
11 & 0.00431 & 2.610 \\
12 & 0.00427 & 2.631 \\
19 & 0.00270 & 2.783 \\
20 & 0.00256 & 2.800 \\
\hline
\end{tabular}

\subsection{3 $L_{c}$ Equation for Routine Operations}

The $\mathrm{L}_{\mathrm{c}}$ value for routine chest and whole body counts is based on $\mathrm{a} \leq 5 \%$ probability of obtaining a false positive result from the measurement. That is, one or more radionuclide results from the measurement are expected to exceed the Lc value when no radionuclide activity is present. The $\mathrm{L}_{\mathrm{c}}$ equation from Equation 1 in ANSI N13.30 (ANSI 1996) is shown below:

$$
\mathrm{Lc}=\left(\mathrm{K}_{\text {alpha }} \times \mathrm{S}_{0}\right)+(\Delta \mathrm{B} \times \mathrm{B})
$$

where $\mathrm{K}_{\mathrm{alpha}}$ is the abscissa of the standardized normal distribution corresponding to the probability level of (1-alpha) and alpha is the false positive probability, $\mathrm{S}_{0}$ is the standard deviation in the net count rate of a subject with no added analyte, $\Delta \mathrm{B}$ is the maximum expected fractional difference between the background of the subject being counted and the background for the subject estimated from the appropriate blank (baseline error estimate), and B is the number of background counts in the ROI. $\Delta \mathrm{B}$ is assumed to be zero for the in vivo radiobioassay results since the background is estimated from the same worker's spectrum that is used to calculate the activity result. Therefore,

$$
\mathrm{L}_{\mathrm{c}}=\left(\mathrm{K}_{\mathrm{alpha}} \times \mathrm{S}_{0}\right)
$$

where $\mathrm{S}_{0}=\left(\mathrm{S}_{\mathrm{B}}^{2}+\mathrm{S}_{\mathrm{B} 0}^{2}\right)^{1 / 2}$ where $\mathrm{S}_{\mathrm{B}}^{2}$ is the standard deviation in the count of a subject with no activity and $\mathrm{S}_{\mathrm{B} 0}^{2}$ is the standard deviation of the blank used to estimate the background. 
In practice, $\mathrm{S}_{0}$ is estimated as $[2 \mathrm{x} \text { (Background counts) }]^{1 / 2}$ and $\mathrm{K}_{\mathrm{alpha}}=2.12$ for routine chest counting and whole body counting applications involving up to 3 radionuclides in the library.

Hence, Equation (6.3) can be rewritten as:

$$
\begin{aligned}
L_{c} & =2.12 \times \operatorname{SQRT}(2 \times \text { Background counts }) \\
& =3 \times \operatorname{SQRT}(\text { Background counts })
\end{aligned}
$$

The estimated background counts used to calculate the $\mathrm{L}_{\mathrm{c}}$ values are assumed to be representative of an unexposed population. However, if the members of the workforce have small amounts of activity in or on them at levels between zero and the Lc value, this can result in an apparent false positive rate greater than the expected value.

\subsubsection{Minimum Detectable Activity}

The MDA value is the level at which activity can be detected in a person with a particular level of confidence (e.g., 95\%). The MDA value is set when the acceptable false positive and false negative rates are established. Decreasing either error rate will, therefore, increase the MDA value unless the other error rate is correspondingly increased. For in vivo measurements, the blank matrix (i.e., the worker) is variable and may contain quantities of manmade or naturally occurring radioactive material; therefore, the MDA, like the $\mathrm{L}_{\mathrm{c}}$, is dependent on the person being measured, as well as on the system-specific parameters. Nonetheless, a measurement system's detection capability can be described by an average MDA value or a range of values. The average MDA values shown in Tables 6.2-6.4 were calculated using Equation (6.6) which is based on Equation 6 on page 22 of ANSI N13.30. (ANSI 1996).

$$
\begin{aligned}
& M D A=\frac{(1+\Delta K) \cdot\left[(2 \cdot \Delta B \cdot B)+2 L_{c}+3\right]}{K \cdot T} \\
& \text { where } \mathrm{L}_{\mathrm{c}}=\text { decision level in counts } \\
& \mathrm{K}=\text { calibration factor } \\
& \mathrm{T}=\text { count time in minutes } \\
& \Delta \mathrm{K}=\text { maximum fractional systematic error estimate } \\
& \Delta \mathrm{B}=\text { baseline error fraction estimate } \\
& \mathrm{B}=\text { background estimate for the ROI }
\end{aligned}
$$

For routine counting $\Delta \mathrm{K}=0.05$ and $\Delta \mathrm{B}=0$. This MDA equation assumes that the values for the false positive probability and false negative probability are equal. This probability value is $1.7 \%$ per nuclide for both routine chest measurements and whole body measurements where results are calculated for up to three radionuclides.

\subsubsection{MDA Values for Chest Counting}

The MDA values, calculated with Equation (6.6), for a 50-minute chest count using four $38 \mathrm{~cm}^{2}$ planar HPGe detectors are tabulated in Table 6.2. 
Table 6.2. Decision Level and MDA Values for Chest Counts

\begin{tabular}{lcccc}
\hline \multicolumn{5}{c}{ Lc and MDA values for routine chest counting systems } \\
\hline Nuclide & $\underline{\text { Lc (Reference Man) }}$ & MDA (Reference Man) & Lc $($ Hanford Man) & MDA (Hanford Man) \\
Am241 & 0.091 & 0.199 & 0.113 & 0.246 \\
Th234 & 0.702 & 1.528 & 0.867 & 1.888 \\
U235 & 0.053 & 0.115 & 0.062 & 0.136 \\
\hline
\end{tabular}

The $\mathrm{L}_{\mathrm{c}}$ value (in counts) used to calculate the MDA is an average $\mathrm{L}_{\mathrm{c}}$ value calculated from worker in vivo measurements that had results less than the Lc value. The MDA values listed for reference man are for a $3.0 \mathrm{~cm}$ chest thickness calculated by inserting the reference male height $(176 \mathrm{~cm})$ and weight $(73 \mathrm{~kg})$ values from ICRP 89 into Equation 4.3 in the appropriate units. The MDA values for the Hanford Man are based on a chest thickness of $3.7 \mathrm{~cm}$ which is the average value for 1899 male workers at Hanford. The MDA varies depending on the subject's actual chest wall thickness (CWT). For example, the ${ }^{241} \mathrm{Am}$ MDA can range from $0.10 \mathrm{nCi}$ for a $1.9-\mathrm{cm} \mathrm{CWT} \mathrm{to} 0.40 \mathrm{nCi}$ for a $6.0-\mathrm{cm} \mathrm{CWT}$.

\subsubsection{LC and MDA Values for Whole Body Counting}

The MDA values for workers weighing less than $200 \mathrm{lbs}$. are tabulated in Table 6.3 and were calculated with Equation (6.6). These values are for a 10-minute scan with the coaxial germanium system described in Section 3.1.2.

Table 6.3. Decision Level and MDA Values for 10-Minute Whole Body Count

\begin{tabular}{ccc}
\hline Nuclide & Decision Level (nCi) & MDA (nCi) \\
\hline Cs-137 & 0.4 & 1 \\
Co-60 & 0.3 & 0.8 \\
Eu-154 & 0.8 & 2 \\
\hline
\end{tabular}

The Lc and MDA values can be higher by $30 \%$ or more for workers weighing greater than $200 \mathrm{lbs}$.

The MDA values, calculated with Equation (6.6), for a 3.33-minute count using the standup counter with $5 \mathrm{NaI}$ detectors described in Section 3.1.1 are tabulated in Table 6.4.

Table 6.4. Decision Level and MDA Values for 3.33 Minute Whole Body Count

\begin{tabular}{ccc}
\hline Nuclide & Decision Level (nCi) & MDA (nCi) \\
\hline Cs-137 & 0.6 & 1.3 \\
Co-60 & 0.6 & 1.2 \\
Eu-154 & 3 & 7 \\
\hline
\end{tabular}

\subsection{Measurement Performance Statistics}

The relative bias, relative precision, false positive results and control charts are tools that are used to monitor the performance of the counting systems. Relative bias and relative precision are evaluated during DOELAP performance testing. The frequency of apparent false positive results is evaluated periodically. The control charts are the primary tool used to monitor the systems' performance relative to the performance at the time of the calibration phantom measurements. In addition to the daily review of the quality control results, data from extended periods are trended to help identify subtle changes in a system's performance. 


\subsubsection{Relative Bias}

Relative bias is defined in ANSI N13.30 (1996) as "The quotient of the bias divided by the expected value." It provides an indication of how close the calculated activity is to the actual activity in the body. Since the actual activity in a person is rarely known, this criterion applies to measurements on suitable phantoms that simulate the body or parts of the body. System relative bias is estimated by replicate counting of appropriate phantoms containing known amounts of radioactive material. Periodic measurements are made of the IVRRF calibration phantoms to verify the calibration factors used for making estimates of activity in the body. Every three years, as part of the Department of Energy Laboratory Accreditation Program (DOELAP) the relative bias is determined from the direct radiobioassay performance-testing session.

The relative bias statistic $(\mathrm{Br})$ for a given system and nuclide is calculated as follows:

$$
\begin{aligned}
& \mathrm{B}_{\mathrm{r}}=\Sigma\left\langle\mathrm{B}_{\mathrm{ri}} / \mathrm{n}\right\rangle \\
& \text { where } \\
& \mathrm{B}_{\mathrm{ri}}=\frac{\left(\mathrm{A}_{\mathrm{i}}-\mathrm{A}_{\mathrm{ai}}\right)}{\mathrm{A}_{\mathrm{ai}}}
\end{aligned}
$$

and $A_{i}$ is the value of the $i$ th measurement in a category being tested, $A_{a i}$ is the actual quantity in the test phantom for the $\mathrm{i} t \mathrm{th}$ measurement; and $\mathrm{n}$ is the number of phantom measurements (i.e., $\Sigma \mathrm{n}_{\mathrm{i}}$ ). The acceptable relative bias stated in DOE-STD-1112-98 is -0.25 to +0.50 .

\subsubsection{Relative Precision}

The relative precision is defined in ANSI N13.30 (1996) as the relative dispersion of the values of $\mathrm{B}_{\mathrm{ri}}$ from the mean and is calculated as follows:

$$
\mathrm{S}_{\mathrm{b}}=\frac{\sqrt{\Sigma\left(\mathrm{B}_{\mathrm{ri}}-\mathrm{B}_{\mathrm{r}}\right)^{2}}}{(\mathrm{n}-1)}
$$

where $\mathrm{n}$ is the total number of replicate measurements (i.e., $\Sigma \mathrm{n}_{\mathrm{i}}$ ), and $\mathrm{S}_{\mathrm{b}}$ is the relative precision statistic. For performance testing purposes, $S_{b}$ shall be in absolute value $\leq 0.4$ for measurements at the minimum testing level (MTL) values listed in ANSI N13.30 (1996). Checks of the measurement precision are also performed as part of DOELAP performance testing.

\subsubsection{False Positive Tracking}

A total false positive rate of $\leq 5 \%$ for each measurement is expected for routine whole body and chest counts. Periodic estimates are made of the false positive rates for the measurement systems. The percentage of apparent false positive results is typically in the range of $3 \%$ to $7 \%$ except for ${ }^{137} \mathrm{Cs}$ which has a somewhat higher value. Low level external contamination, radon progeny interference, and intakes that result in activity levels above zero but below the Lc value can result in apparent elevated false 
positive rates. Evaluations are made to identify the reasons for marked departures from the expected false positive rates

\subsubsection{Measurement Quality Control}

The efficiency, gain conversion, and resolution of the routine measurement systems are checked daily before and after the workers are counted. Control charts are generated to monitor the systems' performance using these parameters. The control chart limits are established as part of the primary calibration process. They are used to determine whether a counting system's performance is comparable to the performance at the time of the last calibration. This correlation is used to show that the calibration factors are applicable to an individual's measurement. The results from the daily quality control measurements are reviewed at least quarterly to monitor the long-term performance of the routine counting systems.

\subsection{Measurement Uncertainty}

The IVMP is designed to minimize the uncertainty in the activity estimates through a combination of accurate calibrations, measurement quality control, established counting procedures, and the use of qualified staff with proper training.

The statistical aspects of in vivo measurements of people are not as precise and as easily determined as those for measuring radioactive sources and samples in well-defined geometries. The uncertainty in the in vivo measurement process can be classified as having two components designated Type A and Type B in accordance with NIST 1297. A Type A uncertainty is estimated using statistical analysis of a series of observations. A Type B uncertainty is determined using methods other than the statistical analysis of a series of observations.

The following is a list of some of the significant sources of uncertainty related to the in vivo measurement of radioactive material in people.

- Uncertainty in the gross, background, and net count rates,

- Uncertainty in the efficiency calibration (includes counting uncertainty, uncertainty in activity content of phantom, positioning uncertainty),

- Uncertainty in half-life values,

- Counting geometry uncertainties,

- Difference in activity distribution between subject and calibration phantom,

- Difference in size, shape, and composition between person and calibration phantom,

- Uncertainty in chest thickness estimate,

- Positioning uncertainty.

It is not appropriate to try and report an uncertainty for each in vivo result that includes all possible sources of uncertainty. The magnitude of some uncertainties (e.g., activity distribution, person size, shape, and composition departure from calibration phantom) is simply unknown for routine measurements and including them would attach an unrealistic magnitude of uncertainty. Thus not all possible sources of uncertainty are included in the reported measurement uncertainty. The in vivo results reported by the IVMP staff include the uncertainties associated with counting statistics, calibration (includes counting statistics, source activity, and phantom positioning), and a 5\% (whole body) or 10\% (chest) systematic uncertainty. 
Estimates of the magnitude of a propagated uncertainty are presented in Table 6.5 for whole body and chest measurements. The uncertainties are associated with activity estimates slightly above the decision level values shown in Tables 6.2 and 6.3. The uncertainty values are based on the relative standard deviation associated with the mean of the calculated activity distributions. Although the calculated distributions are not exactly normal they are approximately normal so that the standard deviation provides an adequate indication of the uncertainty.

The Crystal Ball software was used to calculate the uncertainties associated with the gross counts, background counts, chest wall thickness for chest counts, calibration factors, and positioning of the detectors relative to the worker. Crystal Ball is an Excel add-in Monte Carlo simulation package that is used to generate probability distributions and sensitivity analysis of the variables. The gross and background uncertainties were modeled using a Poisson distribution, the CWT and positioning factors were modeled assuming a normal distribution. The parameters and assumptions used for the uncertainty analyses are maintained as program files. The analysis was performed for routine measurement times of 50 minutes for chest measurements and 10 minutes for whole body measurements.

The chest count analyses were performed for chest wall thicknesses of $2 \mathrm{~cm}, 4 \mathrm{~cm}$, and $6 \mathrm{~cm}$. The CWT uncertainty was propagated through the exponential calibration equation to provide the uncertainty associated with the calibration. The ${ }^{239} \mathrm{Pu}$ activity distribution at $6 \mathrm{~cm} \mathrm{CWT} \mathrm{deviates} \mathrm{most} \mathrm{markedly} \mathrm{from}$ a normal distribution but the standard deviation is still taken as an adequate approximation for the uncertainty at large CWT values. The uncertainties and assumed distributions associated with chest wall thickness estimates, gross count rate, background count rate, efficiency calibration factor, and positioning were included as inputs for the analysis. The Crystal Ball software also generates a sensitivity analysis showing the relative contributions to the total uncertainty from the different sources of uncertainty. The uncertainty associated with the gross and the background count rates contributed $>80 \%$ to the total uncertainty for ${ }^{241} \mathrm{Am}(59.5 \mathrm{keV})$ and ${ }^{235} \mathrm{U}(185.7)$ at all $\mathrm{CWT}$ values. The CWT uncertainty increased from $2.0 \%$ at $2 \mathrm{~cm} \mathrm{CWT} \mathrm{to} 15 \%$ at $6 \mathrm{~cm} \mathrm{CWT} \mathrm{for} \mathrm{these} \mathrm{radionuclides.} \mathrm{At} \mathrm{the}{ }^{239} \mathrm{Pu}$ energy $(17 \mathrm{keV})$, the relative contribution from the chest wall thickness uncertainty increases with increasing CWT as expected and is the main contributor comprising $50 \%$ of the total uncertainty at $6 \mathrm{~cm} \mathrm{CWT.} \mathrm{Figure} 6.1$ contains an example of a sensitivity chart for plutonium.

The uncertainty associated with the measurement of ${ }^{137} \mathrm{Cs}$ in the whole body with an array of five coaxial germanium detectors was modeled to provide an indication of the uncertainty associated with quantifying activity at or near the decision level for fission and activation products in general.

The uncertainty associated with the activity distribution and the uncertainty associated with the anatomical and compositional differences between the worker and the calibration phantoms were not included in the Crystal Ball analysis. These sources can contribute a significant amount of uncertainty to the measured value. For example, measurements were made of a $100 \mathrm{nCi}$ point source of ${ }^{241} \mathrm{Am}$ positioned on the lateral edge of a lung in a torso phantom. The result was calculated based on a calibration derived from a uniform activity distribution in the lungs. The calculated value underestimated the point source activity by a factor of five. This is of course an extreme case but provides an indication of the magnitude of uncertainty that could be encountered. 
Table 6.5. Estimates of Propagated Uncertainty for Routine In Vivo Measurements near the Decision Level

\begin{tabular}{cccc}
\hline Radionuclide & $\begin{array}{c}\text { Chest wall thickness } \\
(\mathbf{c m})\end{array}$ & $\begin{array}{c}\text { Uncertainty } \mathbf{a} \text { 1 sigma } \\
\text { confidence level }\end{array}$ & Measurement type \\
\hline${ }^{239} \mathrm{Pu}(17 \mathrm{keV})$ & 2 & $70 \%$ & Lung \\
& 6 & $90 \%$ & \\
${ }^{241} \mathrm{Am}(59.5 \mathrm{keV})$ & 2 & $64 \%$ & Lung \\
& 4 & $67 \%$ & \\
& 6 & $72 \%$ & Lung \\
${ }^{235} \mathrm{U}(185.7 \mathrm{keV})$ & 2 & $66 \%$ & \\
& 4 & $67 \%$ & Whole Body \\
\hline
\end{tabular}

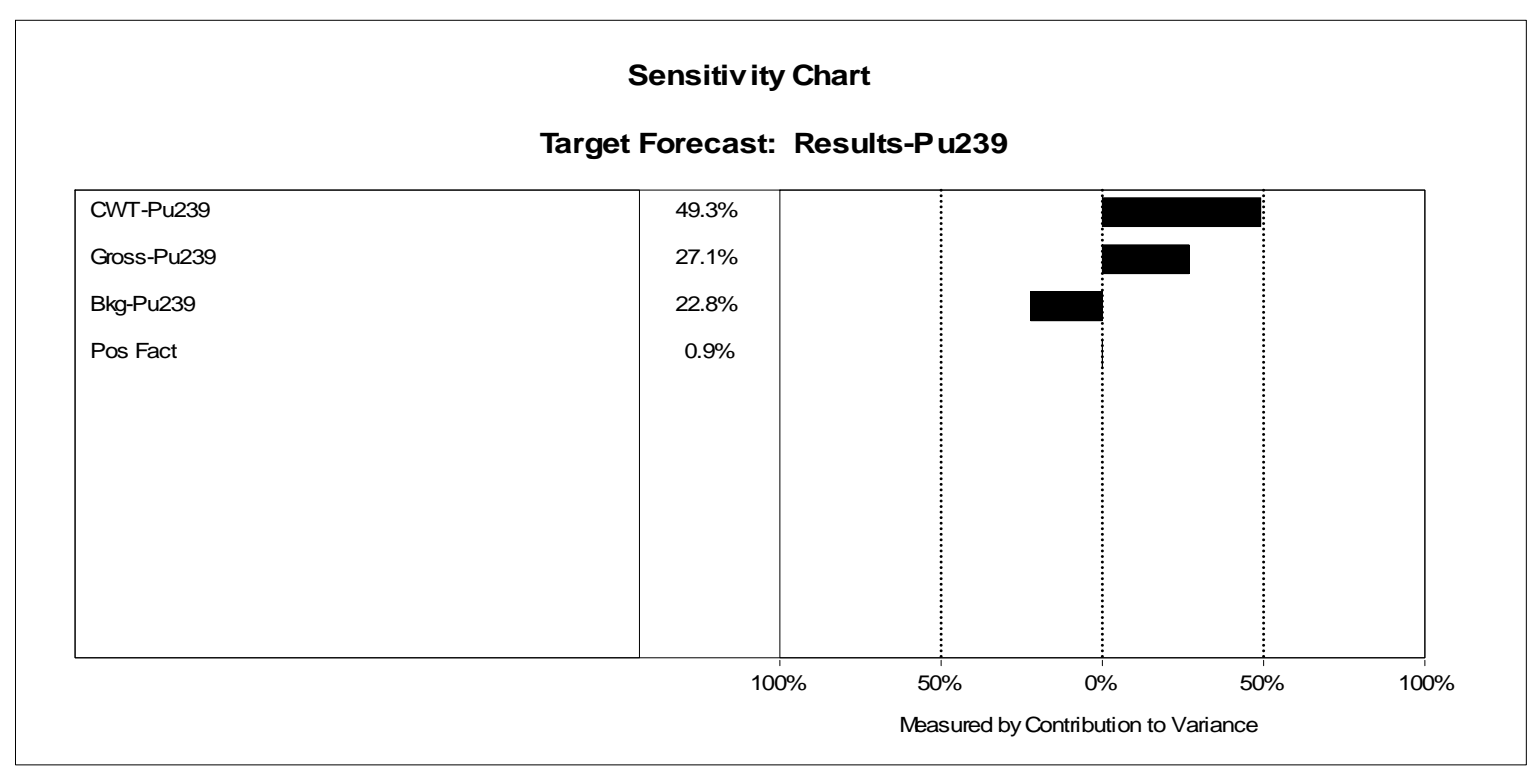

Figure 6.1. Sensitivity Analysis for Measurement near the Decision Level of ${ }^{239} \mathrm{Pu}$ in Lungs at 6 cm CWT

Measurements were made of a ${ }^{152} \mathrm{Eu}$ check source placed at various locations on the top surfaces of a bottle-manikin absorption (BOMAB) phantom containing no radioactive material to obtain an indication of the effect of activity distribution on the uncertainty for scanning whole body measurements. The ${ }^{152} \mathrm{Eu}$ activity of the point source was underestimated by $85 \%$ with the source on the head, $250 \%$ with it on the abdomen, and $1100 \%$ with the source on the right shin. This data was based on the activities calculated at $779 \mathrm{keV}$ and $1408 \mathrm{keV}$. In actual practice, a stationary measurement using a single detector would be used to quantify discrete activity such as a small puncture wound. However, the point source 
measurement results obtained at the different locations provides a useful indication of the possible magnitude of the bias if the activity were extremely localized and quantified based on a whole body distribution.

For less localized activity, measurements were made of a combination of BOMAB sections spiked with radioactive material and sections with no added activity. The bias ranged from $5 \%$ or less for activity in an arm section to $80 \%$ for activity localized in a lower leg section. Pelvic activity was estimated within 10\% suggesting that ingested activity in the gastrointestinal tract might be reasonably estimated with a calibration based on a uniform distribution in the body.

As an indication of uncertainty associated with size, there is a $25 \%$ difference in efficiency obtained with a $170 \mathrm{lb}$. BOMAB phantom compared to a $225 \mathrm{lb}$. BOMAB. Preliminary modeling results indicated an efficiency value at least another $25 \%$ lower for workers weighing $\geq 400 \mathrm{lbs}$.

Estimates of uncertainty for lung counting based on possible differences such as tissue composition, lung size, bone size and composition between the workers and calibration phantom are not readily measured for routine counting and have not yet been quantified. To illustrate a possible minimum value for the uncertainty from these sources, the counting efficiency at different CWT values shows a change of a factor of 6 at $17 \mathrm{keV}, 2$ at $59.5 \mathrm{keV}$, and 2 at $185.7 \mathrm{keV}$ between CWT values of $1.6 \mathrm{~cm}$ and $4.1 \mathrm{~cm}$.

\subsection{References}

American National Standards Institute (ANSI). 1996. Performance Criteria for Radiobioassay. ANSI Standard N13.30, New York.

Currie, L. "Lower Limit of Detection: Definition and Elaboration of a Proposed Position for Radiological Effluent and Environmental Measurements." Washington, D.C. U.S. Nuclear Regulatory Commission; NUREG/CR-4007; 1984.

Genie-VMS Abacos Plus User's Manual, Model 480686. Canberra Industries, Inc., September 2000, Meriden, CT.

Genie VMS Spectroscopy System Users Manual, Model 48-0198, Canberra Industries, Inc., September 1995, Meriden, CT.

NIST Technical Note 1297. Guidelines for Evaluating and Expressing the Uncertainty of NIST Measurement Results, 1994. National Institute of Standards and Technology, Gaithersburg, MD.

Peak Search Program Algorithm (07-0064), Canberra Industries, Inc., March 1985, Meriden, CT.

Spectroscopy Applications Algorithms and Software Verification and Validation Manual (07-0368-03), Canberra Industries, Inc., November 1993, Meriden, CT.

U.S. Department of Energy. 1998. DOE Laboratory Accreditation Program for Radiobioassay. DOESTD-1112-98. Washington. D.C.

VMS Software Service Newsletter, Volume 14, Canberra Nuclear Products Group. March 1994. 
IN VIVO MONITORING PROGRAM MANUAL

\author{
PNL-MA-574
}

SECTION 7.0

IN VIVO MEASUREMENT RECORDS 


\section{Contents}

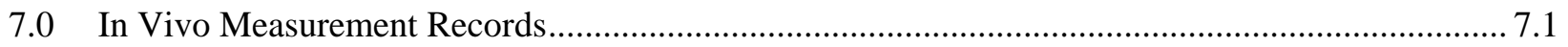

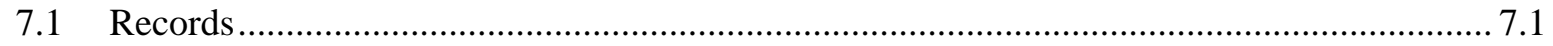

7.2 Handling and Disposition of In Vivo Measurement Records................................................. 7.3

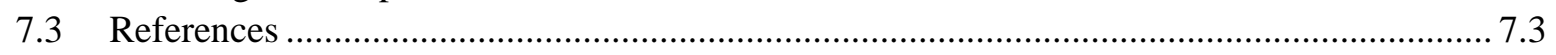




\subsection{In Vivo Measurement Records}

The In Vivo Monitoring Program (IVMP) records are generated and maintained in accordance with the Radiation \& Health Technology (R\&HT) Quality Assurance Program Plan (QAPP), the Records Management (RM) subject area in the How Do I system (HDI), and PNL-MA-870, procedures AP-10, R\&HT Backup Protection of Electronic Records Policy and AP-13, Preparing and Changing Records. These documents implement the applicable guidance and requirements found in 10CFR835; ANSI N13.30, Performance Criteria for Radiobioassay; DOE 1324.2A, Records Disposition; ANSI N13.6 (1999), Practice for Occupational Radiation Exposure Records Systems; and DOE-STD-1112-98, DOE Laboratory Accreditation Program for Radiobioassay. This section provides an overview of the records generated from the process of performing in vivo measurements to detect radioactive material in Hanford workers. The IVMP File Plan describes the program records, their retention periods and ultimate disposition. The File Plan is reviewed annually and updated as necessary.

A moratorium on the destruction of all records generated under the Pacific Northwest National Laboratory (PNNL) 1830 contract is in effect. Due to pending litigation, the Department of Energy (DOE) directed PNNL to cease the destruction of 1830 contract records. This includes records that have met their retention requirements, whether they are maintained in offices or have been sent to storage.

\subsection{Records}

The IVMP generates records specific to each worker measurement, technical support records that provide objective evidence that the measurement results are valid and administrative records that document how the program achieves valid measurement results.

The worker-specific records include the items listed in Section 5.6.5.3 in the DOE technical standard DOE-STD-1112-98 and are listed below. As such these records are considered to be radiological records since they are maintained for specific individuals. The inclusion of this information in the record satisfies the requirements in 10CFR835.702, Individual Monitoring Records. These records are subject to the provisions of the Privacy Act and are handled accordingly.

The information that should be included or referenced in the direct radiobioassay measurement report for the permanent record taken from Section 5.6.5.3 in DOE-STD-1112-98 is listed below.

(1) subject identification;

(2) date, time, and reason for analysis;

(3) identification of radionuclides for which the subject was analyzed and other radionuclides detected;

(4) type of measurement--lung, whole body, thyroid, etc.;

(5) quantification of the amount of radionuclides (whether positive, negative or zero) measured in each part of the body counted;

(6) estimates of counting uncertainty and the total propagated uncertainty (includes counting and other random and/or systematic uncertainties) at the 1-sigma confidence level (Note: total propagated 
uncertainty not included on report; see Chapter 6.0 of this manual for estimates of total propagated uncertainty);

(7) value of the decision level $\left(\mathrm{L}_{c}\right)$ and minimum detectable activity (MDA) in the same units as the results and the expected Type I and Type II errors (Chapter 6.0 of this manual contains the information on expected Type I and Type II errors);

(8) the net count rate and standard error for measurements made for the purposes of screening only, and not for a quantitative measurement (Note: all measurement results for Hanford workers are quantitative and reported in units of nCi);

(9) the value of the customer-specified or service laboratory action level for prompt notification (this may be documented elsewhere and need not appear in the report) (Note: the $L_{c}$ value is used as the action level for notification of the points of contact for Hanford contractors);

(10) the signature of the person responsible for the report or his/her designee.

The paper report generated for each measurement contains most of the information listed above and serves as the record for the measurement. Information not found in the report is contained in other program documents. The paper report is initialed and dated by the technical reviewer. The report also includes peak search results, identification of the energy and efficiency calibrations used, nuclide library used, nuclide specific efficiencies, and the gross, background, and net counts in each of the regions of interest (ROI) for the reported nuclide results.

The channel-by-channel listings of the spectral data are not considered to be program records and in most cases will continue to be retained only in electronic form. The results and spectral data are contained in the same configuration files generated for each measurement. However, paper records of the channel-by-channel spectral data are usually only printed for measurement results that exceed the $\mathrm{L}_{\mathrm{c}}$ value. The adequacy of the software to generate the proper spectral data has been demonstrated by successfully meeting the Department of Energy Laboratory Accreditation Program (DOELAP) performance testing criteria, is verified each day through the measurement quality control program, and by periodic verification of the Abacos analysis algorithms based on phantom measurements.

The technical support records include calibration records for each measurement system, measurement quality control records for each system, maintenance and repair of equipment, and pertinent research and development records. These records provide the documentation to demonstrate how the measured count rate is converted to an estimate of activity in the body and that the calibration factors are valid for each measurement. Typically, these records are not subject to provisions of the Privacy Act.

The administrative records include the operating procedures, administrative procedures (PNL-MA870), Quality Assurance Program Plan, contractual statements of work, and results from audits and surveillances, quality problem report documentation, and program manuals including this manual and the IVMP procedures manual (PNL-MA-554). The PNL-MA-870 manual and the R\&HT QAPP are retained as part of the R\&HT File Plan and are cross-referenced in the IVMP File Plan. Typically, these records are not subject to provisions of the Privacy Act. 


\subsection{Handling and Disposition of In Vivo Measurement Records}

Paper records are generated for each worker measurement, quality assurance (QA) measurement, counting system calibration, quarterly trend analyses of the daily QA results, ancillary measurements to support operations, and other measurements as directed by the Program Manager (PM) and Operation Supervisor (OS). Electronic files associated with these records may also be retained for information purposes but are not considered to be records. The paper records that are generated are stored on an interim basis at the In Vivo Radioassay and Research Facility (IVRRF) and are periodically sent to Records Management (RM) for permanent storage as volume warrants. Unlike the electronic files, the paper records related to worker measurements contain the initials of the technical reviewer and the date of the review. The paper records sent to RM are described on a Records Storage Form including the specific box number they reside in to facilitate future recall. The Records Storage Forms are maintained as program records.

The electronic files generated by the IVMP computer system are stored on disk. All files on the disk are copied to tape nightly. The older files are eventually archived to compact disk (CD) and deleted from the computer system hard disk. A duplicate CD containing the files is also made. The computer disks, tapes, and CD media are protected from unauthorized access as well as fire damage.

The electronic files of the measurement results include demographic information about the individual, the measurement results, analysis parameters, and the channel-by-channel spectral data. Results and spectral data for calibration, quality control and research counts are also electronically stored. For each measurement of a Hanford worker, an electronic file containing the activity, decision level, uncertainty, analysis parameters, and pertinent demographic information is generated. The activity, decision level, uncertainty and demographic information are transferred to the Radiological Exposure System (REX) database. The REX database is maintained as part of the Hanford Radiological Records Program. The files are uploaded from the IVMP workstation to the REX database over the PNNL local area network. The REX database is a tool used to store and access the occupational radiation exposure records for Hanford workers. Changes to the in vivo measurement data after submittal to the REX database are made by submitting a REX change request form to the database administrator.

Results from the in vivo measurements are maintained in the REX database. In addition, copies of the measurement results from 1977 through November 21, 1999 are stored on CDs. Prior to 1977, there are microfilm copies of the result sheets.

\subsection{References}

American National Standards Institute (ANSI). 1999. Practice for Occupational Radiation Exposure Records Systems. ANSI Standard N13.6. New York.

American National Standards Institute (ANSI). 1996. Performance Criteria for Radiobioassay. ANSI Standard N13.30, New York.

Pacific Northwest National Laboratory (PNNL). R\&HT Quality Assurance Program Plan. Richland, Washington. (Internal manual)

Pacific Northwest National Laboratory (PNNL). PNL-MA-554. R\&HT In Vivo Monitoring Program Procedures Manual. Richland, Washington. (Internal manual) 
Pacific Northwest National Laboratory (PNNL). PNL-MA-870. R\&HT Administrative Processes.

Richland, Washington. (Internal manual)

How Do I Standards-Based Management System, Pacific Northwest National Laboratory.

U.S. Department of Energy. 1988. Records Disposition. DOE 1324.2A, Washington, D.C.

U.S. Department of Energy. 1998. DOE Laboratory Accreditation Program for Radiobioassay. DOESTD-1112-98. Washington, D.C.

10CFRPart 835. 1993. U.S. Department of Energy (DOE), “Occupational Radiation Protection.” U.S. Code of Federal Regulations. 
Appendix A

History of Count Types and Minimum Detectable Activity 


\section{Contents}

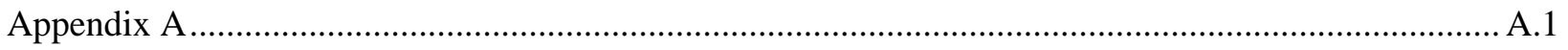

History of Count Types and Minimum Detectable Activity .................................................................. A.1

A.1 Incorporation of Solid State High-Resolution Detectors ................................................... A.2

A.2 Early Whole Body Counts and Hand Calculation of Data................................................ A.3

A.3 Nuclear Data ND-2200 System and Nine-Track Tape Unit ..................................................3

A.4 Data General NOVA Computer System, 1981-1986, and NDCON Software........................ A.3

A.5 Hewlett Packard HP-9000 Series 500 Computer, 1986-1995, and EXEC Software ................ A.4

A.6 Detector Codes and Body Code Combinations..................................................................... A.4

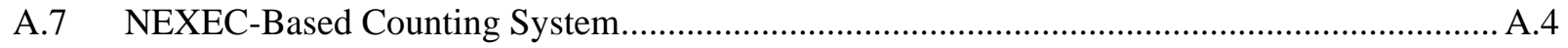

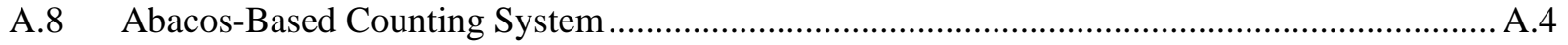

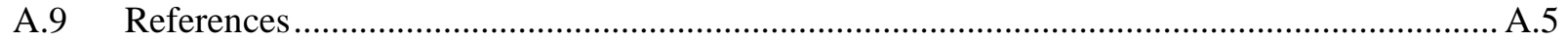




\section{Appendix A}

\section{History of Count Types and Minimum Detectable Activity}

In general, there has been a gradual shift from preset levels of reportability to reporting of anything detected. In the 1970s, for whole body counting the level of reporting was set at $1 \%$ of a maximum permissible body burden (MPBB), as mandated by NBS Handbook 69 and ICRP Publication 2. The MPBB was defined as "the Body Burden of Radionuclide(s) which, under any combination of circumstances, is not expected to cause appreciable bodily harm to the average individual during his lifetime." In this environment, detecting ${ }^{60} \mathrm{Co}$ in an individual in an amount below $100 \mathrm{nCi}$ was reported as a "trace of ${ }^{60} \mathrm{Co} . "$

Such a "trace" of ${ }^{60} \mathrm{Co}$ would grossly interfere with the calculation of the five standard nuclides (i.e., ${ }^{137} \mathrm{Cs},{ }^{65} \mathrm{Zn},{ }^{24} \mathrm{Na},{ }^{54} \mathrm{Mn},{ }^{40} \mathrm{~K}$ and a low energy region). In keeping with the fact that calculations were done by hand, the calculation sheet would reflect "trace of ${ }^{60} \mathrm{Co}$ " for any amount from detectable to 99 $\mathrm{nCi}$. Additionally, since the standard nuclide calculation could not handle the presence of the ${ }^{60} \mathrm{Co}$, the results for ${ }^{60}$ Co were "starred out." This meant only that the routine results calculation was not valid. Note that it is not proper to conclude that the count was not valid. Because this amount was not considered "reportable," the individual was not required to shower or change into coveralls for a recount. Consequently, the amount calculated was not necessarily in the person but could have been on the clothing. Although the actual amount was calculated to verify that it was below the $1 \%$ of an MPBB, the actual result was not recorded. If more than one nuclide was present, each was calculated and the percentage of an MPBB calculated. If the sum did not exceed 1\%, the nuclides were again reported as only a "trace."

In spite of the high reporting levels, the detection capabilities of that era were very respectable as compared to present practice. Table A.1 shows the minimum detectable activity (MDA) values typical of the 1970's measurements and the percentage of the MPBB it represents.

Early counts had the spectra printed out channel by channel on fanfold paper containing a unique serial number, together with the subject's initials, last name, payroll number, and date. After a holding period of about a year, paper tapes containing count data were sent to storage.

Source and background counts were taken in the morning and the background spectra were stored in the analyzer. Each subject count thereafter had this background subtracted from it. Thus, the spectra printed to paper tape were already background-subtracted. Regions of interest (ROI) were summed from the paper tapes and the sum was transferred to the calculation sheet, which was sent to Radiological Records when completed. 
Table A.1. Whole Body Counter Detection Capabilities

\begin{tabular}{ccc}
\hline Nuclide & Detection Level & Fraction of MPBB \\
\hline${ }^{51} \mathrm{Cr}$ & $50 \mathrm{nCi}$ & $5 \times 10^{-5}$ \\
${ }^{60} \mathrm{Co}$ & $4 \mathrm{nCi}$ & $4 \times 10^{-5}$ \\
${ }^{65} \mathrm{Zn}$ & $0.8 \mathrm{nCi}$ & $1.3 \times 10^{-5}$ \\
${ }^{137} \mathrm{Cs}$ & $0.6 \mathrm{nCi}$ & $2 \times 10^{-5}$ \\
${ }^{24} \mathrm{Na}$ & $0.3 \mathrm{nCi}$ & $4.2 \times 10^{-5}$ \\
${ }^{59} \mathrm{Fe}$ & $2.0 \mathrm{nCi}$ & $1 \times 10^{-4}$ \\
${ }^{22} \mathrm{Na}$ & $1.0 \mathrm{nCi}$ & $1 \times 10^{-4}$ \\
${ }^{131} \mathrm{I}$ & $20 \mathrm{pCi}$ (thyroid) & $1.4 \times 10^{-4}$ \\
& $0.5 \mathrm{nCi}$ & $3.6 \times 10^{-3}$ \\
${ }^{239} \mathrm{Pu}$ & $0.1 \mathrm{nCi}$ (wound counter) & $2.5 \times 10^{-3}$ \\
\hline
\end{tabular}

Some later tapes were generated by an analyzer capable of summing channels in such a manner that the first channel contained the actual channel contents, but each succeeding channel contained its own value plus the sum of each previous channel. By this method, the integral of a region could be quickly calculated by subtracting the value of the lower channel of the region of interest from the value of the highest channel in the ROI. Visual analysis and search for peaks on such integral tapes are quite laborious.

On the calculation sheet, the higher-energy regions were multiplied by predetermined factors and subtracted from the lower-energy regions to act as a condensed version of spectrum stripping. The net counts in each ROI were then multiplied by a calibration factor, and the results for four standard nuclides were reported in microcurie units. The four standard radionuclides at this time were ${ }^{40} \mathrm{~K},{ }^{137} \mathrm{Cs}$, ${ }^{65} \mathrm{Zn}$, and ${ }^{24} \mathrm{Na}$. A fifth region, termed the "God Only Knows" (GOK) region, from 200 to $400 \mathrm{keV}$, was also recorded. GOK was recorded as net counts per minute (cpm).

In this earlier era (from 1969 to about 1989) with lower counting loads, subjects who were positive often received additional counts. These counts verified the results reported but were not necessarily themselves reported officially. The practice was to report the best available estimate, not all supporting measurements that made up the estimate. For example, linear scans were often done with several collimated 2-x-2-in. NaI detectors in a side-by-side geometry to help determine the location of the activity. Some measurements were also done with phoswich detection systems. These counts did not yield numerical results for reporting but might prompt a note or a comment that most of the activity appeared to be in the lung region. A high-resolution detector might be used to help identify the nuclides present and to determine the ratio of one nuclide to another in a subject, but the actual quantification was probably done on the more sensitive NaI detector, which had a more established geometry and calibration factors.

\section{A.1 Incorporation of Solid State High-Resolution Detectors}

Phoswich detector systems were used extensively in testing and phantom counting in the 1970s but were never adopted as a routine counting system. Four 3/8-x-5-in. NaI detectors, counting from the front and back, gave sensitivity similar to two Phoswich detectors. They were also more suitable for routine 
counting by virtue of not having as intricate a series of set-up procedures. For routine counting, the four 5-in. NaI detectors were replaced by solid-state intrinsic HPGe planar detectors, which are still in use today.

The introduction of high-resolution detectors, first to assist in identifying and then for both identifying and quantifying, greatly increased the confidence and ease of analyzing mixtures of nuclides.

In about 1983, the introduction of planar, intrinsic, thin window detector arrays to lung counting also greatly simplified the calculational algorithm, in addition to allowing more positive identification of the deposited nuclide.

\section{A.2 Early Whole Body Counts and Hand Calculation of Data}

The 1-meter arc chair geometry using a 4-x-9-in. NaI detector, and the shadow-shielded sled counter using a 4-x-11-in. detector were in operation from 1960 to about 1980. Both systems used quite similar calibration factors. Localized activity on some counts could yield different results due to the geometrical differences between the two systems.

Four standard nuclides were routinely calculated $\left({ }^{40} \mathrm{~K},{ }^{137} \mathrm{Cs},{ }^{65} \mathrm{Zn},{ }^{24} \mathrm{Na}\right)$ and a fifth "GOK” region of 100 to $200 \mathrm{keV}$. The resolution of the NaI detector in the extremely sloped GOK region was poor and was used as an indication only if a higher-than-normal sum was encountered.

Any peaks apparent in the spectra, other than the five routine regions, were handled by the nonroutine calculation procedure using $80 \%$ of the photopeak. In this case, a ROI encompassing $80 \%$ of the peak height was used to calculate an area that was $80 \%$ of the full photo-peak area. This calculation was based on the average assumption that $20 \%$ of a peak is due to background contributions.

\section{A.3 Nuclear Data ND-2200 System and Nine-Track Tape Unit}

During the 1970's spectra plus a unique tagword were written to a nine-track magnetic tape in binary coded decimal format. This tape was delivered to the Federal Building where the spectra were read into the computer and merged with alphanumeric, individual identification data that had been entered separately through a terminal at the 747A Building, connected to the Federal Building by telephone modem. The computer at the Federal Building would do the routine calculation, write spectra to microfiche and a consolidated magnetic tape, and plot unusual spectra.

Under this procedure, spectra showing trace or greater amounts would have been detected visually at the time the count was done, and a nonroutine hand calculation would have been done on the spectra. The results from these counts were called up immediately to the exposure evaluator and the result sheet sent up to the Federal Building for inclusion in the subject's file.

\section{A.4 Data General NOVA Computer System, 1981-1986, and NDCON Software}

From 1981 to 1986, spectra and identifying alphanumeric data were stored on the Data General NOVA computer and calculations were done immediately following the count. Space on the computer disk was limited, and the data were not in a database but in separate spectra and result files. At weekly intervals, spectra and results were taken to the Federal Building, where a computer would store results for access by the records section and exposure evaluators. The spectra were consolidated from weekly into 
monthly tapes and a copy of the consolidated tape returned to the program staff, in addition to the copy kept at the Federal Building as the archive.

Paper punched tapes brought spectra from remote or mobile counters into the system for processing. The system also stored calibration tapes resulting from studies using the new anthropomorphic chest phantom developed at Lawrence Livermore National Laboratory. This phantom replaced the Masonite projection chest phantom that was used for calibrating chest counting systems up to 1982.

\section{A.5 Hewlett Packard HP-9000 Series 500 Computer, 1986-1995, and EXEC Software}

The EXEC database held spectra along with alphanumeric data and results. The measurement results were calculated with algorithms developed in house. It was capable of storing roughly 1 year of data for immediate access. In addition, multiple users had simultaneous access to data. Calculations were now proliferating at a much greater rate due to conversion to high resolution systems and the increased number of measurements performed.

EXEC was replaced by NEXEC in 1995. NEXEC was a client server configuration using an IBM RISC 6000 for the server and distributed processing with personal computers as the clients. NEXEC utilized an Oracle database management system to store, manipulate, retrieve and transfer data.

\section{A.6 Detector Codes and Body Code Combinations}

Equation numbers are a designation of a unique calculating subroutine. They began with the implementation of the HP-9000 computer and the EXEC computer program in 1986. Under NDCON on the Data General NOVA computer, subroutines were also used to do the calculations, but only with the adoption of EXEC on the HP-9000 was each subroutine given this unique identifier. This code simplified the task of keeping track of the greater number of calculational algorithms being introduced.

Beginning January 1, 1992, the in vivo measurement results were reported using a decision level $\left(\mathrm{L}_{\mathrm{c}}\right)$ calculation based on guidance in ANSI N13.30 instead of the MDA to determine whether a detectable amount of activity was present. All detector codes at this time were changed to consist of two consecutive letters instead of a letter and a number, as had been done previously.

\section{A.7 NEXEC-Based Counting System}

The NEXEC-based counting system was placed into routine operation in 1995 and was used through October 21, 1999, when a hard disk failure caused the system to be unusable. The NEXEC system was a client-server based configuration. The server was an IBM RS6000 with an Oracle relational database. The clients were IBM-PC that were interfaced to the RS6000 over the Hanford local area network. The algorithms for the ROI type of calculations were executed on the PC. The spectral data were acquired using a Digital Equipment Corporation Workstation 3100 running the basic Canberra Genie spectroscopy software. Both the channel-by-channel spectral data and the measurement results were saved to the Oracle database.

\section{A.8 Abacos-Based Counting System}

Since October 22, 1999, the Abacos-Based Counting System software has been used for routine operations. The software was purchased from Canberra Industries and is run on a Digital Equipment 
Corporation Alphastation running the VMS operating system. The Abacos analysis programs differ from the methods used for the EXEC and NEXEC systems. However, comparable results from phantom measurements were obtained with both types of software. This was required to obtain technical equivalence from DOELAP in order to place the Abacos software into service.

The calibration files in the File Plan contains copies of the energy, resolution, and efficiency calibrations used for the data analysis. The user manuals and other vendor technical information provide explanations of how the software operates and were used to develop the operating procedures.

\section{A.9 References}

ICRP Publication 2. Report of Committee II on Permissible Dose for Internal Radiation. 1959. International Commission on Radiological Protection. Pergamon Press. London.

NBS Handbook 69. Maximum Permissible Body Burdens and Maximum Permissible Concentrations of Radionuclides in Air and in Water for Occupational Exposure. National Bureau of Standards Handbook 69. Washington, D.C. 1959. 
Appendix B

Historical Summary of Count Types, Body Codes, and Detector Codes 


\section{Contents}

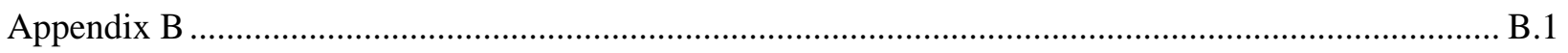

Historical Summary of Count Types, Body Codes and Detector Codes ............................................... B.1 


\section{Appendix B}

\section{Historical Summary of Count Types, Body Codes, and Detector Codes}

The in vivo measurement records contain codes used to describe the type of measurement and the types of detectors used for the measurement. The body code provides an indication of the organ or tissue being measured. For example, a lung measurement was designated with a body code of 03 while a whole body measurement was designated as 01, except for specialized measurements. In Table B.1 there are multiple detector codes for a single body code that reflects the different types of detectors used at different times.

Table B.2 duplicates some of the material in Table B.1 but adds equation numbers and so marks a distinct enhancement in identifying the algorithm used in the calculation. Table B.2 reflects the operating environment up to November 1999.

Beginning on October 22, 1999, the acquisition, analysis, and storage of the in vivo data was performed with the Abacos Plus software. The Abacos Plus software is a commercial off-the-shelf product purchased from Canberra Industries. Table B.3 contains the reason codes, body codes, and detector codes that have been in use since October 22, 1999. 
Table B.1. In Vivo Measurement Information by Codes and Count Lengths

\begin{tabular}{|c|c|c|c|}
\hline $\begin{array}{l}\text { Body } \\
\text { Code }\end{array}$ & $\begin{array}{l}\text { Detector } \\
\text { Code }\end{array}$ & $\begin{array}{l}\text { Count } \\
\text { Length }\end{array}$ & Description of Count Types and Notes \\
\hline \multicolumn{4}{|c|}{ Lung Measurements } \\
\hline 03 & B & 2000 & $\begin{array}{l}\text { Four 5-x-3/8-in. NaI detectors with beryllium windows. Most results } \\
\text { based on estimated chestwall thickness unless noted with a comment. }\end{array}$ \\
\hline 03 & $\mathrm{~F}$ & 2000 & Same as above but different set of detectors. \\
\hline 03 & $\mathrm{R}$ & 2000 & Three high purity germanium (HPGe) detectors. \\
\hline 03 & $\mathrm{~T}$ & 1000 & Five HPGe detectors. \\
\hline 03 & V or V1 & 1000 & $\begin{array}{l}\text { Six HPGe detectors. Almost all positive results are based on actual } \\
\text { chestwall thickness, as measured by ultrasound unless noted otherwise. If } \\
\text { a correction has been applied to the activity in the lung, a comment is } \\
\text { noted, such as "Corrected for Skeletal Activity." }\end{array}$ \\
\hline 03 & $\mathrm{XA}, \mathrm{YA}$ & 3000 & Four 38-cm² HPGe detectors. \\
\hline \multicolumn{4}{|c|}{ Whole Body Measurements } \\
\hline 01 & A & 600 & One NaI detector and $18 \%$ GeLi in chair configuration. \\
\hline 01 & G & 600 & One NaI detector - Shadow shield mobile counter. \\
\hline 01 & $\mathrm{H}$ & 600 & One NaI detector- Shadow shield. \\
\hline 01 & $\mathrm{~S}$ & 600 & One NaI detector with two GeLi’s shadow shield. \\
\hline 04 & $\mathrm{~S}$ & 300 & $\begin{array}{l}\text { Used to show the distribution of localized activity in the body. } \\
\text { Quantified from the " } 01 \text { - S” whole body count with three 5-minute } \\
\text { counts over the head, chest, and abdomen. Percentage values are } \\
\text { designated in the comment. }\end{array}$ \\
\hline 01 & $\mathrm{U}$ or $\mathrm{U} 1$ & 200 & $\begin{array}{l}\text { Five NaI detectors/standing configuration. A total of } 512 \text { channels, } \\
\text { where first } 256 \text { channels are from sum of all five detectors and second } \\
256 \text { channels are from detector behind the lung only. }\end{array}$ \\
\hline 01 & $\mathrm{U} 2$ & 200 & $\begin{array}{l}\text { Five NaI detectors/standing configuration, with } 1024 \text { channels } \\
\text { ( } 256 \text { channels each over head, lung, abdomen, and leg areas). }\end{array}$ \\
\hline 01 & UA, UB & 200 & $\begin{array}{l}\text { Five NaI detectors/standing configuration, with } 1024 \text { channels } \\
\text { ( } 256 \text { channels each over head, lung, abdomen, and leg areas). }\end{array}$ \\
\hline 01 & IA & 1200 & $\begin{array}{l}\text { Scanning measurement using four or five coaxial HPGe detectors. } \\
\text { Detectors located under subject lying in a supine position. }\end{array}$ \\
\hline 01 & IB & $600 / 1200$ & $\begin{array}{l}\text { Scanning measurement using seven coaxial HPGe detectors. Detectors } \\
\text { located under subject lying in a supine position. }\end{array}$ \\
\hline
\end{tabular}


Table B.1. (continued)

\begin{tabular}{|c|c|c|c|}
\hline $\begin{array}{l}\text { Body } \\
\text { Code }\end{array}$ & $\begin{array}{l}\text { Detector } \\
\text { Code }\end{array}$ & $\begin{array}{l}\text { Count } \\
\text { Length }\end{array}$ & Description of Count Types and Notes \\
\hline \multicolumn{4}{|c|}{ Head Measurements } \\
\hline 02 & $\mathrm{R}$ & 2000 & Three HPGe detectors on side of head. \\
\hline 02 & Q or Q1 & 3000 & Two HPGe detectors on forehead. \\
\hline 02 & QA & & Two 20-cm² planar HPGe on forehead. \\
\hline 02 & QB & & Two 38- $\mathrm{cm}^{2}$ planar HPGe on forehead. \\
\hline \multicolumn{4}{|c|}{ Liver Measurements } \\
\hline 08 & $\mathrm{R}$ or $\mathrm{R} 1$ & 2000 & $\begin{array}{l}\text { Three HPGe detectors. If a correction was made to the liver from } \\
\text { interfering activity seen in the bone, a comment is entered: "Corrected } \\
\text { for Skeletal Activity." }\end{array}$ \\
\hline 08 & RA & & Three $20-\mathrm{cm}^{2}$ planar HPGe. \\
\hline 08 & $\mathrm{RB}$ & & Two 38-cm² planar HPGe. \\
\hline 08 & $\mathrm{RC}$ & & Three $20-\mathrm{cm}^{2}$ planar HPGe. \\
\hline \multicolumn{4}{|c|}{ Lymph Measurements } \\
\hline 08 & $\mathrm{~J}$ or $\mathrm{J} 1$ & 1000 & One HPGe detector. \\
\hline 08 & JA & 600 & One $20-\mathrm{cm}^{2} \mathrm{HPGe}$ planar detector. \\
\hline \multicolumn{4}{|c|}{ Wound Measurements } \\
\hline & & & $\begin{array}{l}\text { Since most wounds occur on the hand, primary body location used is } \\
\text { "07." }\end{array}$ \\
\hline 07 & $\mathrm{D}$ & 600 & One NaI detector, 2-in. thin crystal beryllium window. \\
\hline 07 & $\mathrm{~J}$ or $\mathrm{J} 1$ & 600 & One HPGe detector. \\
\hline 07 & M or M1 & 600 & One HPGe detector at Emergency Decontamination Facility. \\
\hline 07 & JA & 600 & One $20-\mathrm{cm}^{2} \mathrm{HPGe}$ planar detector. \\
\hline \multicolumn{4}{|c|}{ Thyroid Measurements } \\
\hline 06 & $\mathrm{e}$ & 2000 & One 3-x-3-in. NaI detector, primarily used for ${ }^{131}$ I counting. \\
\hline 06 & $\mathrm{~N}$ or $\mathrm{P}$ & 600 & One phoswich detector, primarily used for ${ }^{125} \mathrm{I}$. \\
\hline 06 & $\mathrm{~L}$ or L1 & 600 & One GeLi for ${ }^{131}$ I counting. \\
\hline 06 & JB & 600 & One $38-\mathrm{cm}^{2}$ planar HPGe, $10 \mathrm{~cm}$ from surface of neck. \\
\hline 06 & Q2 & 600 & Two 20-cm² planar HPGe over thyroid. \\
\hline
\end{tabular}


Table B.2. In Vivo Measurement Information Including Equation Numbers for Current and Historical (pre-1992) Counts

\begin{tabular}{|c|c|c|c|c|}
\hline $\begin{array}{l}\text { Body } \\
\text { Code }\end{array}$ & $\begin{array}{l}\text { Detection } \\
\text { Code }\end{array}$ & $\begin{array}{l}\text { Equation } \\
\text { Number }\end{array}$ & $\begin{array}{l}\text { Norm } \\
\text { Time }\end{array}$ & Description Count Type and Notes \\
\hline \multicolumn{5}{|c|}{ New $L_{c}$ System, Effective 1-1-92 } \\
\hline 01 & UB & 522 & 200 & One 4-x-11-in. and four 4-x-9-in. NaI detectors. \\
\hline 01 & UA & 520 & 200 & One 4-x-11-in. and four 4-x-9-in. NaI detectors. \\
\hline 01 & OA & 531 & 200 & One 4-x-4-x-8-in. and five 4-x-4-x-16-in. NaI detectors. \\
\hline 01 & IA & 141 & 1200 & Four coaxial scan in Utah Room. \\
\hline 03 & VA & 220 & 1200 & $\begin{array}{l}\text { HPGe lung count, six bucket style detectors at } 0.25 \\
\text { keV/channel, Iron Room. }\end{array}$ \\
\hline 03 & VB & 221 & 1800 & $\begin{array}{l}\text { HPGe lung count, four bucket style detectors at } 0.25 \\
\mathrm{keV} / \text { channel, Iron Room. }\end{array}$ \\
\hline 03 & WA & 240 & 1200 & $\begin{array}{l}\text { HPGe lung count, six organ pipe style detectors at } 0.25 \\
\mathrm{keV} / \text { channel, Stainless Steel Room. }\end{array}$ \\
\hline 03 & WB & 241 & 1800 & $\begin{array}{l}\text { HPGe lung count, four organ pipe style detectors at } 0.25 \\
\mathrm{keV} / \mathrm{channel} \text {, Stainless Steel Room. }\end{array}$ \\
\hline 07 & JA & 420 & 600 & Single planar HPGe detector, wound count. \\
\hline 02 & QA & 620 & 3000 & Two planar HPGe detectors, head count. \\
\hline 08 & RA & 920 & 3000 & $\begin{array}{l}\text { Three } 20-\mathrm{cm}^{2} \text { planar HPGe detectors, liver count - Iron } \\
\text { Room. }\end{array}$ \\
\hline 08 & RA & $921,921 \mathrm{~A}$ & 3000 & $\begin{array}{l}\text { Three } 20-\mathrm{cm}^{2} \text { planar HPGe detectors, liver count - Lead } \\
\text { Room. }\end{array}$ \\
\hline 06 & QB & 330 & 600 & Two planar HPGe detectors, thyroid count. \\
\hline 08 & JA & 820 & 1000 & One HPGe planar, lymph node count. \\
\hline 07 & IA & 422 & 600 & One coaxial wound count using $77.1 \%$. \\
\hline 03 & WD & 243 & 1800 & $\begin{array}{l}\text { Four organ pipe style detectors HPGe in Lead Room, 5-5- } \\
94 .\end{array}$ \\
\hline 03 & XA & 260 & 1200 & Four $38-\mathrm{cm}^{2}$ HPGe in Stainless Steel Room. \\
\hline 03 & XA & $261,261 \mathrm{~A}$ & 3000 & Four 38-cm² HPGe in Stainless Steel Room. \\
\hline 03 & YA & $281,281 \mathrm{~A}$ & 3000 & Four $38-\mathrm{cm}^{2}$ HPGe in Iron Room. \\
\hline 02 & QB & $622 \mathrm{~A}$ & 3000 & Two $20-\mathrm{cm}^{2}$ HPGe in Lead Room. \\
\hline 01 & IA & 150 & 1200 & $\begin{array}{l}\text { Five coaxial HPGe detectors in Palmer Room - scanning } \\
\text { measurement. }\end{array}$ \\
\hline 01 & IB & 171A, 171B & 600 & $\begin{array}{l}\text { Seven coaxial HPGe detectors in Palmer Room - scanning } \\
\text { measurement. }\end{array}$ \\
\hline
\end{tabular}


Table B.2. (continued)

\begin{tabular}{|c|c|c|c|c|}
\hline $\begin{array}{l}\text { Body } \\
\text { Code }\end{array}$ & $\begin{array}{l}\text { Detection } \\
\text { Code }\end{array}$ & $\begin{array}{l}\text { Equation } \\
\text { Number }\end{array}$ & $\begin{array}{c}\text { Norm } \\
\text { Time }\end{array}$ & Description Count Type and Notes \\
\hline 01 & IB & 170 & 1200 & $\begin{array}{l}\text { Seven coaxial HPGe detectors in Palmer Room - scanning } \\
\text { measurement. }\end{array}$ \\
\hline 03 & IA & 125 & 1200 & $\begin{array}{l}\text { Five coaxial HPGe detectors in Palmer Room - stationary } \\
\text { measurement. }\end{array}$ \\
\hline \multicolumn{5}{|c|}{ Previously Used Equations Reporting in MDAs } \\
\hline 01 & $\mathrm{U} 2$ & 502 & 200 & $\begin{array}{l}\text { Stand-up whole body count keeping the spectra from the five } \\
\text { detectors separate (four } 4 \text { x } 9 \text { in. and one } 4 \text { x } 11 \text { in.). }\end{array}$ \\
\hline 03 & V5 & 205 & 3000 & $\begin{array}{l}\text { HPGe lung count, four bucket style detectors at } 0.25 \\
\text { keV/channel. }\end{array}$ \\
\hline 03 & V6 & 206 & 2000 & $\begin{array}{l}\text { HPGe lung count, six bucket style detectors at } 0.25 \\
\text { keV/channel. }\end{array}$ \\
\hline 03 & V7 & 207 & 3000 & $\begin{array}{l}\text { HPGe lung count, four organ pipe style detectors at } 0.25 \\
\mathrm{keV} / \text { channel. }\end{array}$ \\
\hline 01 & I3 & 130 & 1200 & $\begin{array}{l}\text { Three coaxial - 60\%, 69\%, } 66.3 \% \text { detector scan in Utah Room } \\
\text { at } 1.5 \mathrm{keV} / \text { channel. }\end{array}$ \\
\hline 01 & I4 & 140 & 1200 & $\begin{array}{l}\text { Four coaxial - 59.4\%, } 69 \%, 66.3 \%, 77.1 \% \text { detector whole body } \\
\text { counter scan in Utah Room at } 1.5 \mathrm{keV} / \text { channel. }\end{array}$ \\
\hline 01 & $\mathrm{O} 2$ & 530 & 200 & $\begin{array}{l}\text { Remote counter, five } 4-x-4-x-16 \text {-in. and a } 4-x-4-x-8-i n . \text { NaI } \\
\text { detector. }\end{array}$ \\
\hline 03 & I4 & 124 & 1200 & $\begin{array}{l}\text { Four coaxial - 59.4\%, } 69 \%, 66.3 \%, 77.1 \% \text { stationary lung } \\
\text { count in Utah Room at } 1.5 \mathrm{keV} / \text { channel. }\end{array}$ \\
\hline 01 & $\mathrm{O} 1$ & None & 1200 & 4-x-4-x-16-in. NaI collimated scan 2-in. slit in Utah Room. \\
\hline 07 & $\mathrm{~J} 2$ & 402 & 600 & $\begin{array}{l}\text { Wound count using one intrinsic HPGe detector at } \\
0.25 \mathrm{keV} / \text { channel. }\end{array}$ \\
\hline 07 & $\mathrm{I} 1$ & 410 & 600 & Wound count using one coaxial detector. \\
\hline 02 & Q2 & 612 & 3000 & Head count using two HPGe on forehead at $0.25 \mathrm{keV} /$ channel. \\
\hline 07 & $\mathrm{~J} 1$ & 400 & 600 & Wound count using one HPGe detector $0.5 \mathrm{keV} /$ channel. \\
\hline 03 & V4 & 202 & 2000 & $\begin{array}{l}\text { HPGe lung count, six bucket style detectors at } 0.25 \\
\text { keV/channel, pre-9/89 calculation. }\end{array}$ \\
\hline 01 & S1 & 100 & 600 & Shadow shield scan count, 6-x-11-in. NaI detector. \\
\hline 01 & G2 & 102 & 600 & Mobile shadow shield, 4-x-11-in. NaI detector. \\
\hline 08 & S1 & 104 & 300 & Shadow shield counter used as clothing counter, 6-x-11-in. NaI. \\
\hline 03 & $\mathrm{I} 2$ & 120 & 900 & $\begin{array}{l}\text { GeLi detectors, } 24 \% \text { and } 34 \% \text {, used for lung counting for high- } \\
\text { energy emitters. }\end{array}$ \\
\hline 03 & V1 & 200 & 2000 & $\begin{array}{l}\text { HPGe lung count, six bucket style detectors at } 0.50 \\
\mathrm{keV} / \mathrm{channel} \text {. }\end{array}$ \\
\hline
\end{tabular}


Table B.2. (continued)

\begin{tabular}{|c|c|c|c|c|}
\hline $\begin{array}{l}\text { Body } \\
\text { Code }\end{array}$ & $\begin{array}{l}\text { Detection } \\
\text { Code }\end{array}$ & $\begin{array}{l}\text { Equation } \\
\text { Number }\end{array}$ & $\begin{array}{l}\text { Norm } \\
\text { Time }\end{array}$ & Description Count Type and Notes \\
\hline 03 & $\mathrm{~V} 2$ & 201 & 2000 & $\begin{array}{l}\text { HPGe lung count, six bucket style detectors at } 0.25 \\
\text { keV/channel. } 201 \text { is not a valid equation. }\end{array}$ \\
\hline 06 & E1 & 300 & 600 & Phoswich thyroid count for ${ }^{125} \mathrm{I}$. \\
\hline 06 & Q2 & 302 & 600 & Thyroid count with two HPGe, $0.25 \mathrm{keV} /$ channel. \\
\hline 06 & $\mathrm{~J} 1$ & 320 & 600 & $\begin{array}{l}\text { Thyroid count with one bucket style detector HPGe (at } \\
\text { Sequim), } 0.5 \mathrm{keV} / \text { channel. }\end{array}$ \\
\hline 01 & $\mathrm{U} 1$ & 500 & 200 & $\begin{array}{l}\text { Stand-up whole body count using sum of five detectors (four } \\
4 \times 9 \text { in. and one } 4 \times 11 \text { in.). }\end{array}$ \\
\hline 02 & $\mathrm{R} 1$ & 600 & 2000 & Head count using three HPGe from one side. \\
\hline 02 & Q1 & 610 & 3000 & $\begin{array}{l}\text { Head count using two HPGe on forehead at } \\
0.5 \mathrm{keV} / \text { channel. }\end{array}$ \\
\hline 08 & D1 & 700 & 600 & Wound count using thin NaI detector. \\
\hline 08 & $\mathrm{~J} 1$ & 800 & 1000 & $\begin{array}{l}\text { Lymph node count using one HPGe detector, } \\
0.5 \mathrm{keV} / \text { channel. }\end{array}$ \\
\hline 08 & J3 & 801 & 1000 & $\begin{array}{l}\text { Lymph node count using one HPGe detector, } \\
0.25 \mathrm{keV} / \text { channel. }\end{array}$ \\
\hline 08 & $\mathrm{R} 1$ & 900 & 1000 & Liver count using three HPGe detectors, $0.5 \mathrm{keV} / \mathrm{channel}$. \\
\hline 08 & R3 & 901 & 1000 & $\begin{array}{l}\text { Liver count using three bucket style detectors HPGe } \\
\text { detectors, } 0.25 \mathrm{keV} / \mathrm{ch} \text { annel. }\end{array}$ \\
\hline 08 & $\mathrm{R} 2$ & 902 & 1000 & $\begin{array}{l}\text { Liver count using three organ pipe style detectors HPGe } \\
\text { detectors, } 0.25 \mathrm{keV} / \mathrm{channel} \text {. }\end{array}$ \\
\hline
\end{tabular}


Table B.3. Measurement Codes in Use Since October 22, 1999

\begin{tabular}{|c|c|c|c|c|c|c|}
\hline \multicolumn{2}{|l|}{ Reason Code } & \multicolumn{2}{|c|}{ IVMP Body Code } & \multirow{2}{*}{\begin{tabular}{c|} 
REX Body Code \\
WBD
\end{tabular}} & \multicolumn{2}{|c|}{ Detector Code } \\
\hline Periodic & PR & Whole Body & WB, WC & & $\begin{array}{l}\text { FX } \\
\text { coax }\end{array}$ & $\begin{array}{l}\text { Five } 120 \% \\
\text { Ge, scan }\end{array}$ \\
\hline Baseline & $\mathrm{BL}$ & Chest & C1 & $\mathrm{CH} 1$ & & \\
\hline Termination & $\mathrm{TM}$ & Chest & $\mathrm{C} 2$ & $\mathrm{CH} 2$ & SU & $\begin{array}{l}\text { Vertical array } \\
5 \text { NaI detectors }\end{array}$ \\
\hline Special & SP & Head & HD & SK1 & DI & Four planar Ge \\
\hline Contractor Request & CR & Wound & $\mathrm{WN}$ & WND & DS & Four planar Ge \\
\hline Recount & RA & Liver & $\mathrm{LV}$ & LIV & $\mathrm{CH}$ & Two planar Ge \\
\hline End Assignment & EA & Lymph & LY & LYM & & \\
\hline Contract Work & 12 & Thyroid & $\mathrm{TH}$ & THY & & \\
\hline Research & 13 & & & & & \\
\hline
\end{tabular}

Table B.4. Routine Radionuclide Library Codes in Use with Abacos Plus Software

Counting System $\quad$ Library codes

Whole Body (since March 20, 2009)

Coaxial germanium

Stand-up counter

Lung (since October 22, 1999)

Iron Room

Stainless Steel Room

Lead Room
W2 (Cs137, K40); W4(Cs137, Co60, Eu154, K40)

S2 (Cs137, K40); S4(Cs137, Co60, Eu154, K40)

CA (Am241); CU (Th234, U235); CC (Am241, Th234, U235);

C5 (U235)

CA (Am241); CU (Th234, U235); CC (Am241, Th234, U235);

C5 (U235)

CA (Am241); CU (Th234, U235); CC (Am241, Th234, U235);

C5 (U235) 
Appendix C

The Hanford In Vivo Radiobioassay and Research Facility

Pamphlet 


\section{THE IN VIVORADIOBIOASSAY AND RESEARCH FACILITY}

\section{LOCATION}

The Hanford In Vivo Radiobioassay and Research Facility is located at 805 Goethals (on the corner of Goethals and Knight Street) in Richland, Washington.

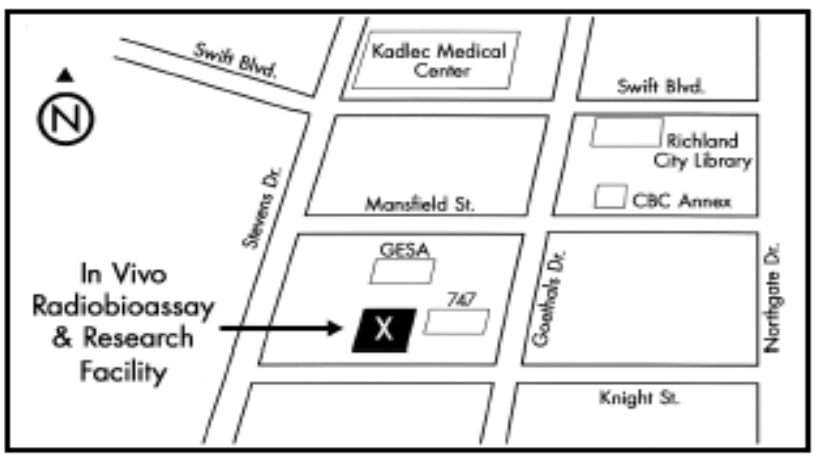

\section{WHAT HAPPENS AT THE IN VIVO RADIOBIOASSAY AND RESEARCH FACILITY?}

WELCOME to the In Vivo Radiobioassay and Research Facility (IVRRF). Hanford workers who are assigned to areas where radioactive materials are processed or stored may be scheduled for exams at this facility. Your examination includes measurements to help assure that the workplace controls established to prevent and limit the intake of radionuclides are working effectively.

Your examination at the IVRRF can detect the presence of very small quantities of radionuclides in your body that emit gamma rays or X-rays. The detectors used at the IVRRF can detect smaller quantities of these radionuclides than the portable survey instruments and the monitors used in your workplace. The detectors do not emit any radiation.

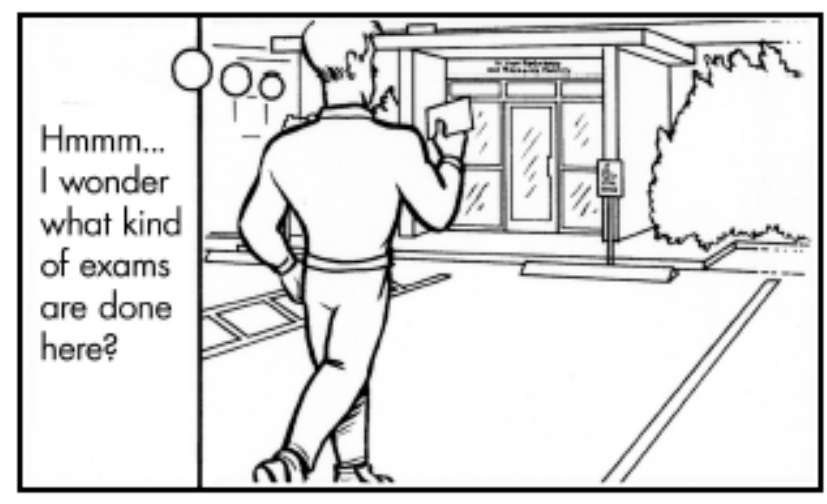




\section{WHEN ARE THE EXAMS SCHEDULED?}

If you are a new employee and have formerly worked with or expect to work with unsealed radioactive materials, you will probably have an examination at the IVRRF before you enter a radiologically-controlled area. These exams establish a "baseline" value against which future results can be compared. You may also have an exam whenever there is a change in your job task, working condition, or the type or form of radionuclides to which you could be exposed. Most Hanford employees who require an in vivo measurement are examined annually. You also may be examined in the event of an accidental exposure.

Most of the results from the in vivo measurements do not calculate detectable amounts of radionuclides that are associated with your work. However, if a result is above the detection level, you may receive one or more additional exams.

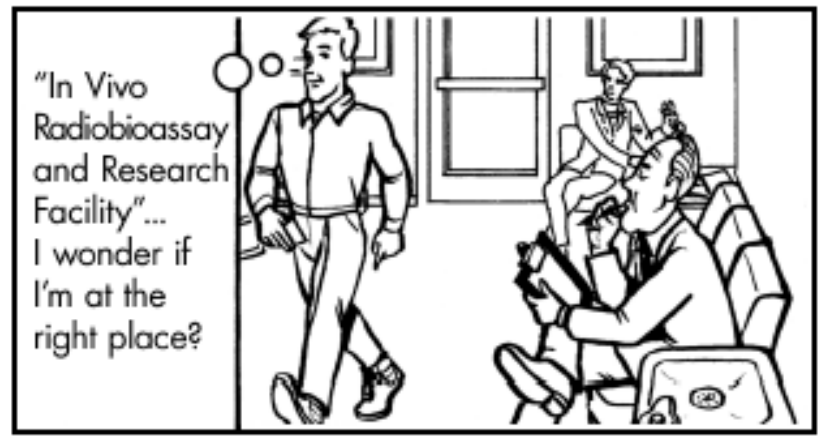

\section{WHAT EXAMS ARE PERFORMED?}

The whole body measurement and the chest measurement are the two most frequently performed examinations at the IVRRF. The whole body measurement is used to determine whether radionuclides (e.g., cesium-137) that emit high-energy photons are detectable in the body. The chest measurement is designed to detect radionuclides in the lungs, in particular those that emit low-energy photon radiations (e.g., uranium and americium-241).

Your radiation protection group establishes a monitoring schedule for you based on the bioassay requirements for your specific job. Many other types of examinations can be performed at the IVRRF; however, they are reserved for special circumstances. The exam results become part of your permanent Hanford radiation exposure record.

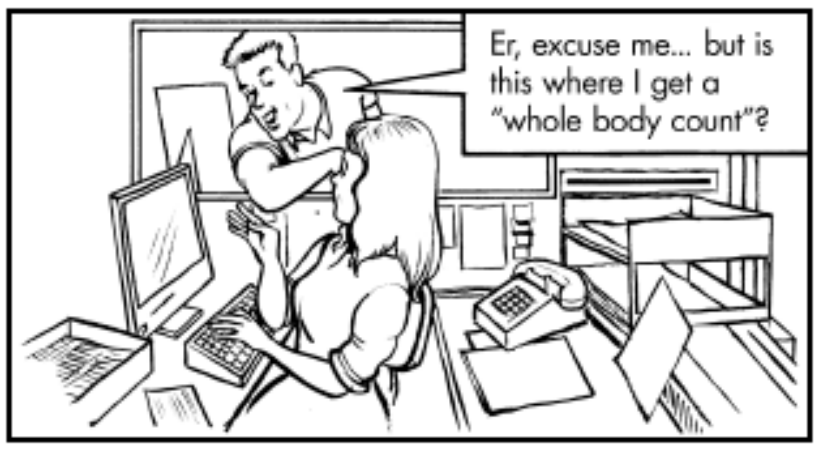


THE TECHNICIAN AT THE IVRRF FIRST REQUESTS THAT YOU COMPLETE THE INFORMATION FORM PROVIDED TO YOU. THE TECHNICIAN MAY ASK YOU TO CHANGE FROM YOUR STREET CLOTHES INTO COVERALLS. COVERALLS ARE WORN BECAUSE SMALL QUANTITIES OF RADIOACTIVE PARTICLES MAY SETTLE ON YOUR CLOTHING. SHOWERS ARE AN ADDITIONAL WAY TO REMOVE TRACE AMOUNTS OF RADIONUCLIDES THAT MAY SETTLE ON HAIR AND SKIN AND INTERFERE WITH THE MEASUREMENT

\section{THE WHOLE BODY COUNT}

The whole body measurements are performed using two different systems. The most frequently performed measurement is done using five germanium detectors that scan the length of the body. The detectors are cooled with liquid nitrogen and are enclosed in a steel room to reduce the background radiation levels. The second type of whole body counter consists of a vertical array of five large sodium-iodide detectors installed in a shielded booth. The lead shielding used for this counter also helps reduce the background radiation levels.

When having a whole body count, you are usually counted in your street clothes initially unless you are also receiving a chest count in which case you will change into coveralls The technician will call you into the counting area in preparation for the measurement(s) . You will be requested to remove your hat, coat, shoes, and possibly other items.

For the scanning measurement, you lie still in a supine position for the 10-minute count. This count is performed in the Palmer Room, which is made from the hull of the battleship U.S.S. Indiana. The Indiana played an important role in several battles in the Pacific theater during World War II.

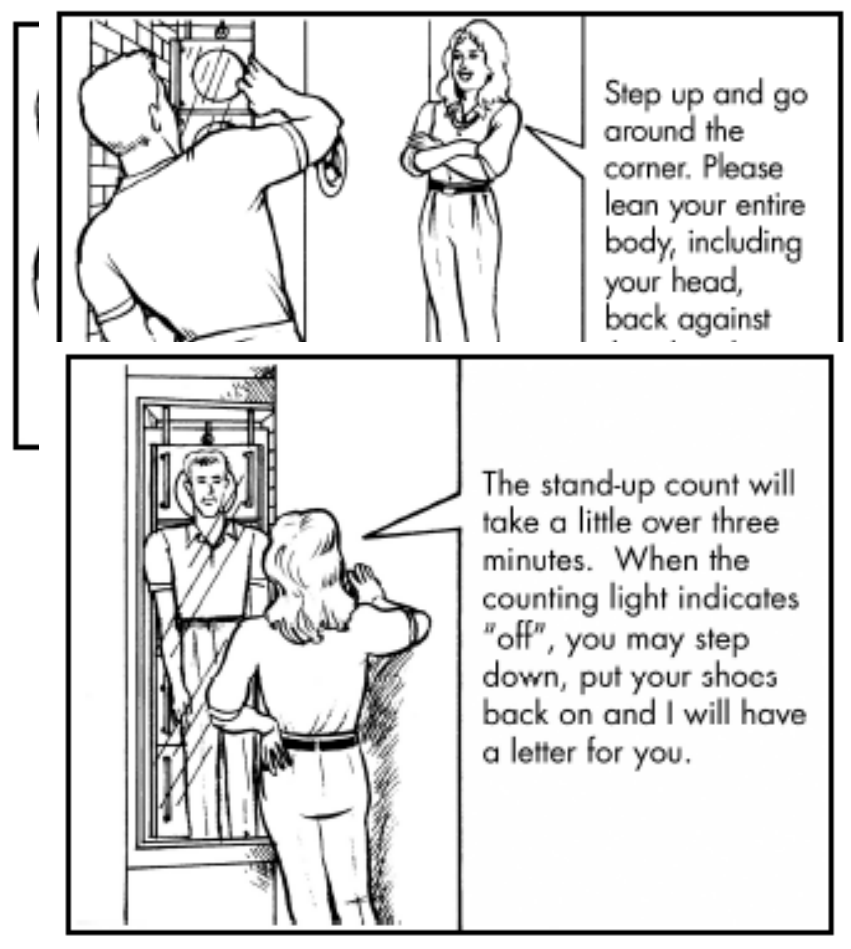


For the stand-up count, you step up into the booth and lean back against the plastic support. The detectors are behind you and the technician adjusts them so your chest is in front of the largest detector. The count takes a little over three minutes, and it is important that you remain stationary to ensure that the results of your exam are accurate and reliable.

If the results from the initial measurement exceed the detection level, a second measurement is usually obtained. The technician will request that you change into coveralls and/or shower before the second measurement.

Your whole body measurement results become part of your Hanford radiation exposure record.

\section{THE CHEST COUNT}

The chest count is used to determine whether a detectable amount of a radionuclide such as americium, uranium, and thorium is present in your lungs. You will be given a pair of coveralls to put on before the measurement. In certain situations (e.g. follow-up to workplace indications of a potential intake) you may be asked to shower before putting on the coveralls. Depending on the system, two to four detectors are positioned on your chest over the lungs. The technician will tell you about the measurement process and answer any questions you might have. The counting times range from 3000 seconds to 4200 seconds.

The chest count is performed in a room shielded with thick steel walls to reduce the background radiation levels. The shielding allows lower levels of radionuclides to be detected in the body. The germanium detectors used for the chest measurements are designed to detect low energy photons. They are cooled with liquid nitrogen.

The results from the chest count become part of your Hanford radiation exposure record.

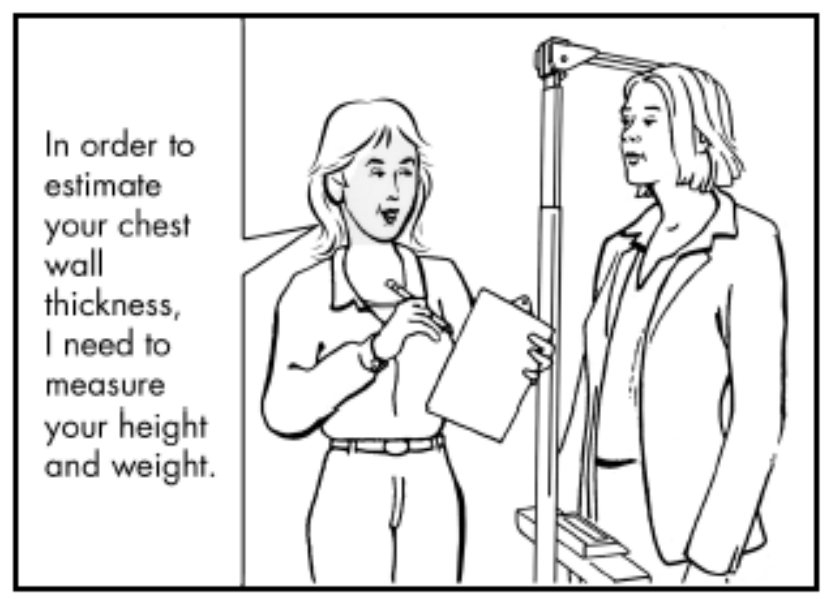


The measurement process is designed to minimize interference from naturally occurring radionuclides; however, trace amounts of external activity from the workplace and naturallyoccurring activity (e.g. radon) can still interfere with the measurement results. Measurement results are also subject to the random statistical nature of counting radionuclides. Unlike counting pennies in a jar which can be counted exactly, the amount of radioactivity can never be determined exactly, but only estimated statistically (similar to determining the probability that you will be struck by lightning tomorrow). This means that about $5 \%$ of the time a count result will calculate a detectable amount of a radionuclide when none is present. The only way to know for sure is to get one or more additional measurements. It is preferable to get the extra count(s) the same day, but if necessary, it can be obtained within a week.

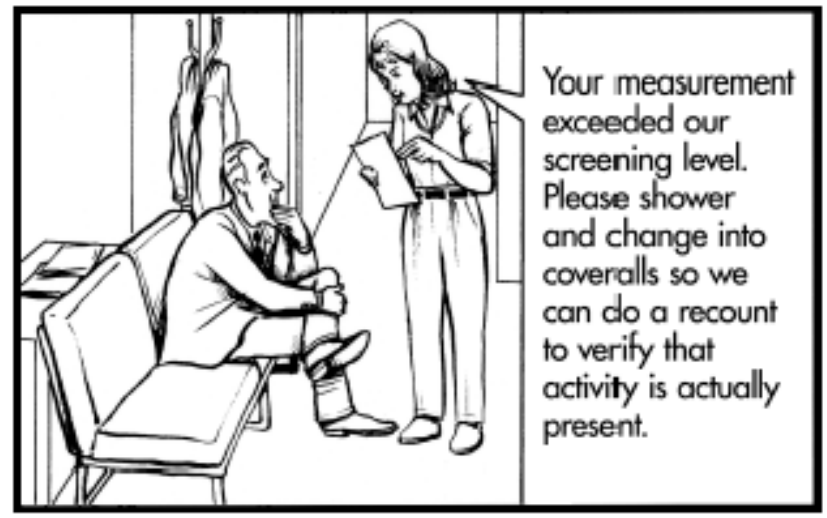

Sometimes the measurement results from the initial and follow-up measurements can indicate a possible internal deposition of radionuclides. In some cases further examinations may also be performed to better quantify the activity, evaluate the distribution in the body, and to

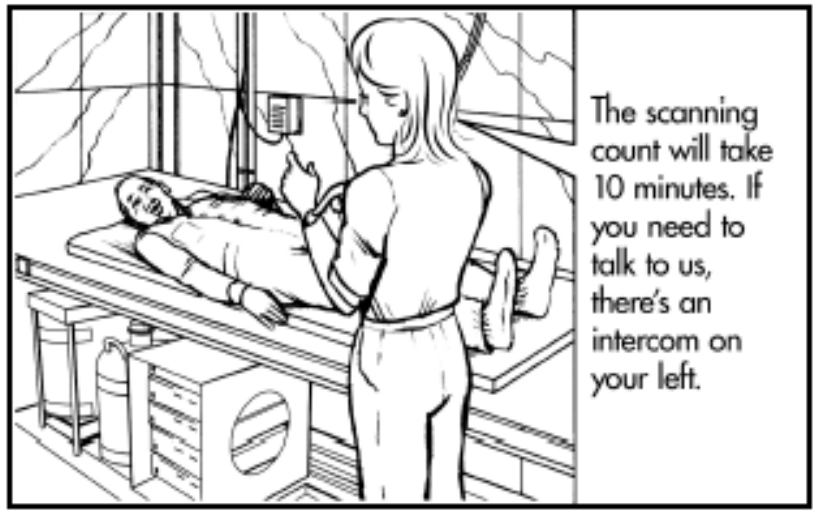

estimate the rate of elimination from the body.

\section{NATURAL RADIOACTIVITY IN THE BODY}

Everyone has radionuclides in their body but they will not make you glow in the dark. 
Potassium-40, the naturally occurring radioactive isotope of potassium is found in all plants and animals. The average person contains about 140 grams of potassium of which $0.01 \%$ is potassium-40. Whole body measurements always detect potassium-40 activity.

Several types of radionuclides are detected at times and may not be directly related to work at Hanford. Radon progeny (e.g., bismuth-214, lead-214) occur naturally in the environment and are detected during many of the measurements at the IVRRF. The highest levels are usually detected during periods of temperature inversions in the winter. The radionuclides may be in the body, on the skin, hair, and clothing.

\section{SOURCES OF OTHER RADIOACTIVE MATERIALS IN THE BODY}

Other radionuclides, such as cesium-137, are not naturally occurring, but exist as a result of fallout from atmospheric testing of nuclear weapons or nuclear accidents such as Chornobyl. Persons eating wild game, such as deer or elk, will occasionally have measurable amounts of cesium-137, which the animals ingest with the vegetation they eat.

Occasionally, technicium-99m, thallium-201, thallium-202, and iodine-131, or other radionuclides may be detected in persons who have had nuclear medicine procedures.

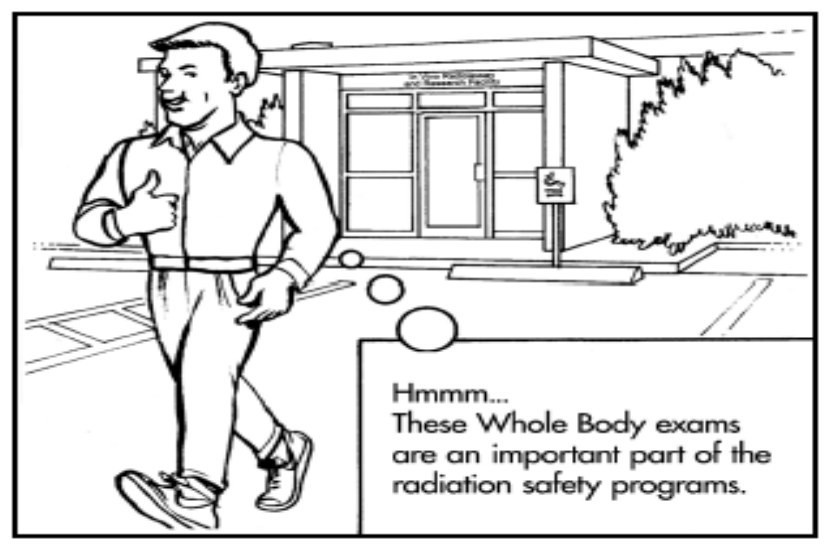

\section{QUESTIONS}

The staff at the IVRRF will answer questions you have about your in vivo exam. You can also contact your company's dosimetry representative. The contact information is provided on the letter you receive when your count is completed. 


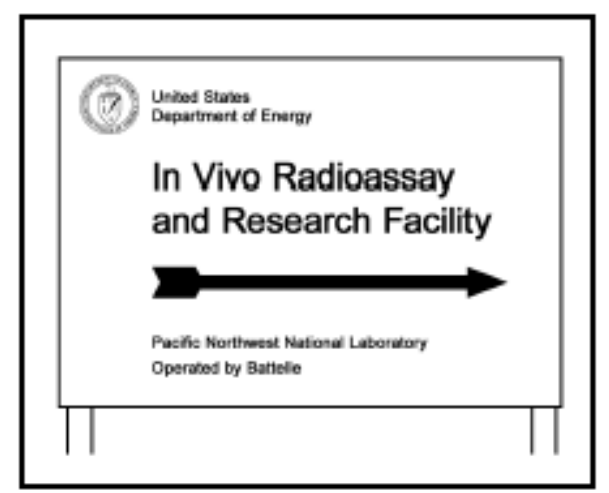

\section{ACKNOWLEDGEMENTS}

Author: Timothy Lynch

Art Director: Nathan Johnson

Cartoonist: Beverly Calicoat 
Appendix D

Acronyms and Abbreviations 


\section{Acronyms and Abbreviations}

\begin{tabular}{|c|c|}
\hline ABCS & Abacos-Based Counting System \\
\hline $\mathrm{ADC}$ & analog to digital converter \\
\hline AIM & acquisition interface module \\
\hline ANSI & American National Standards Institute \\
\hline ВОМАВ & bottle-manikin absorption (phantom) \\
\hline CD & compact disk \\
\hline CDTe & cadmium telluride \\
\hline CWT & chest wall thickness \\
\hline DOE & U.S. Department of Energy \\
\hline DOELAP & Department of Energy Laboratory Accreditation Program \\
\hline DSA & digital spectrum analyzer \\
\hline ENBACK & environmental background \\
\hline $\mathrm{eV}$ & electron volt \\
\hline FET & field effect transistor \\
\hline FP & fission product \\
\hline FWHM & full width at half maximum \\
\hline Ge & Germanium \\
\hline GOK & God Only Knows (region) \\
\hline HRRP & Hanford Radiation Records Program \\
\hline HDI & How Do I \\
\hline HPGe & High Purity Germanium \\
\hline IAEA & International Atomic Energy Agency \\
\hline
\end{tabular}




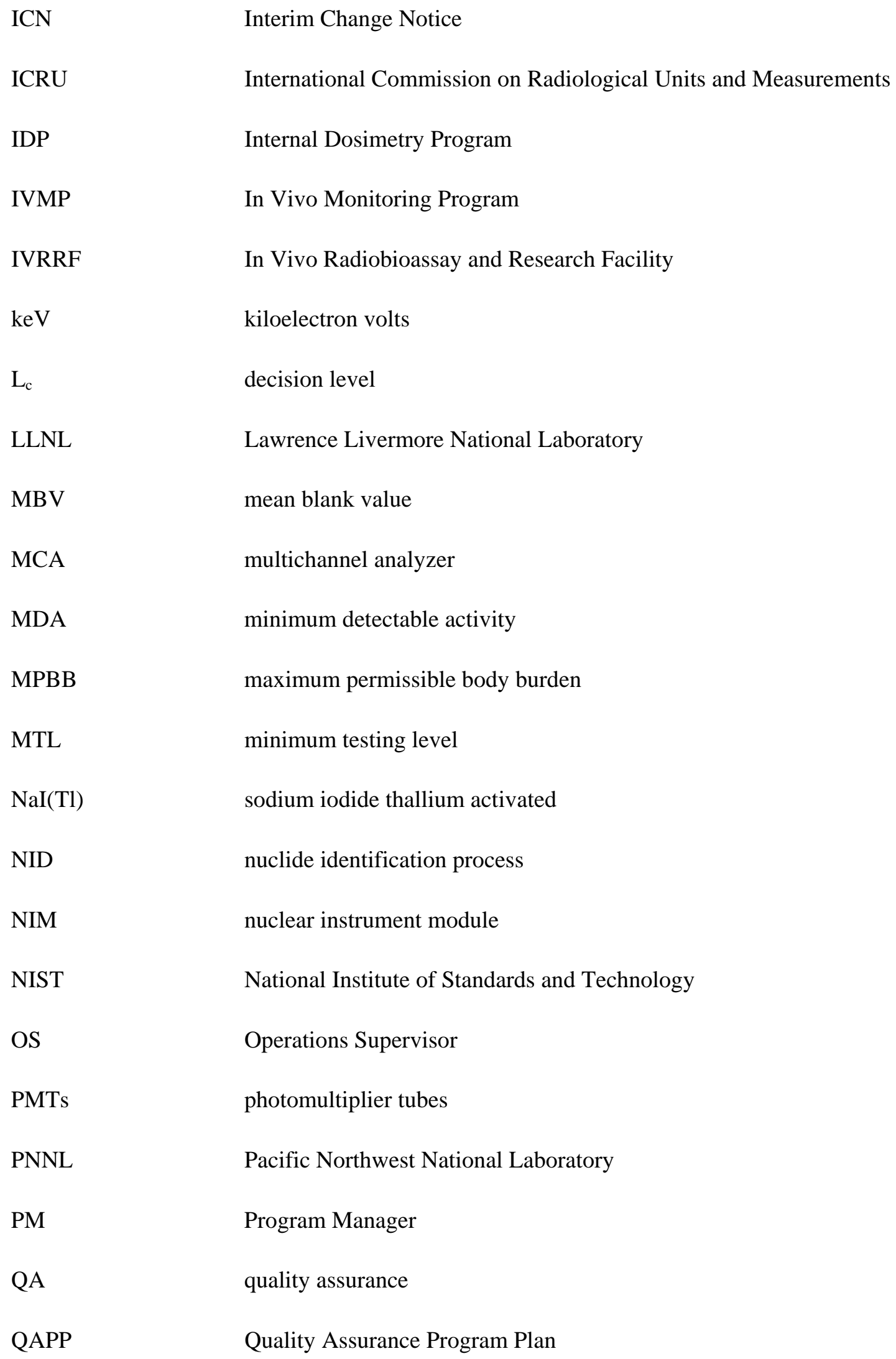


QC

R\&HT

RAM

REX

ROI

$\mathrm{RM}$

USTUR quality control

Radiation and Health Technology

random-access memory

Radiation Exposure System

regions of interest

Records Management

U.S. Transuranium and Uranium Registries 